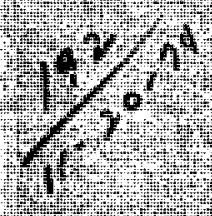

tem $+1 \mathrm{Hin}$

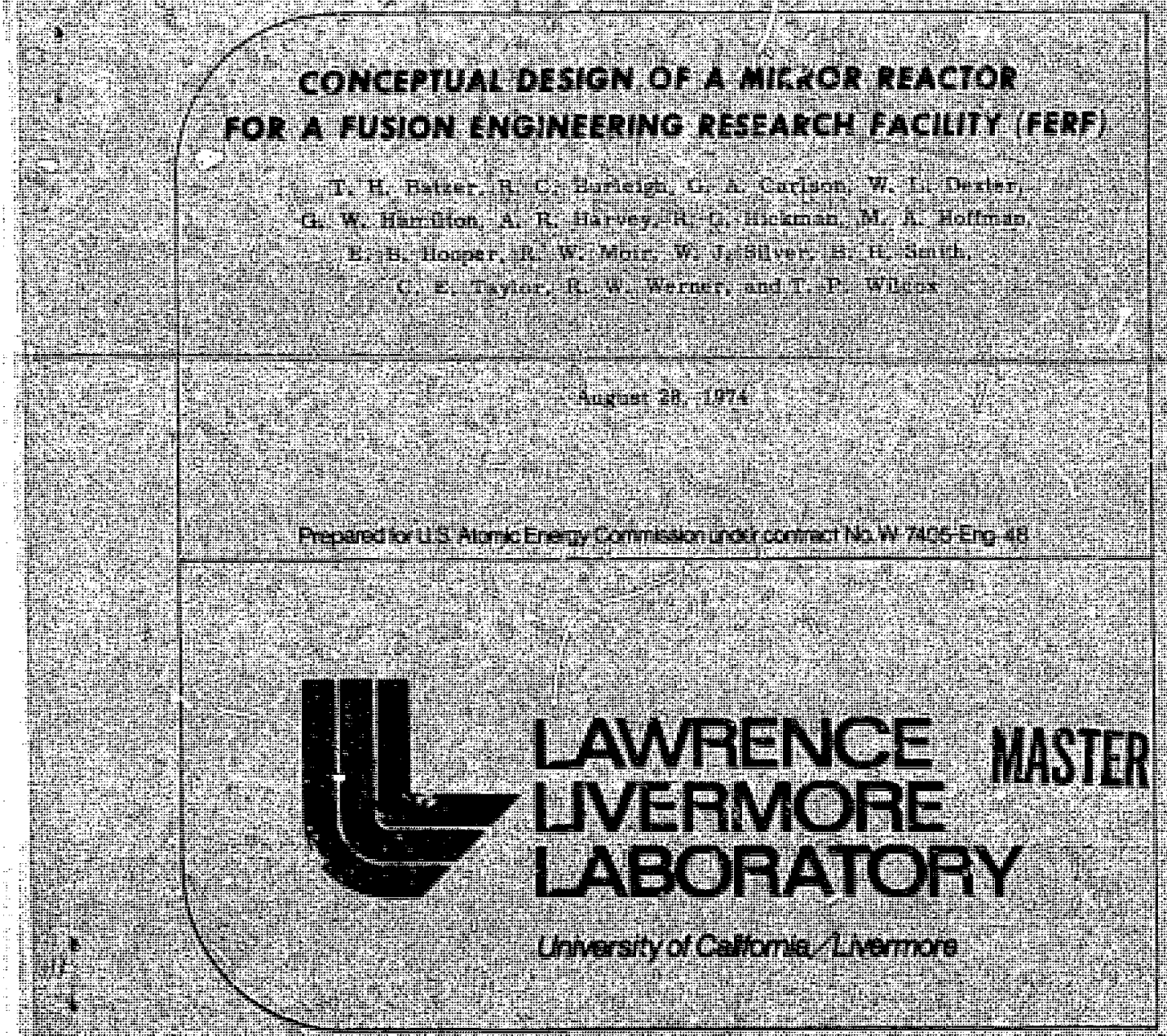

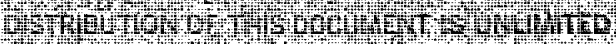




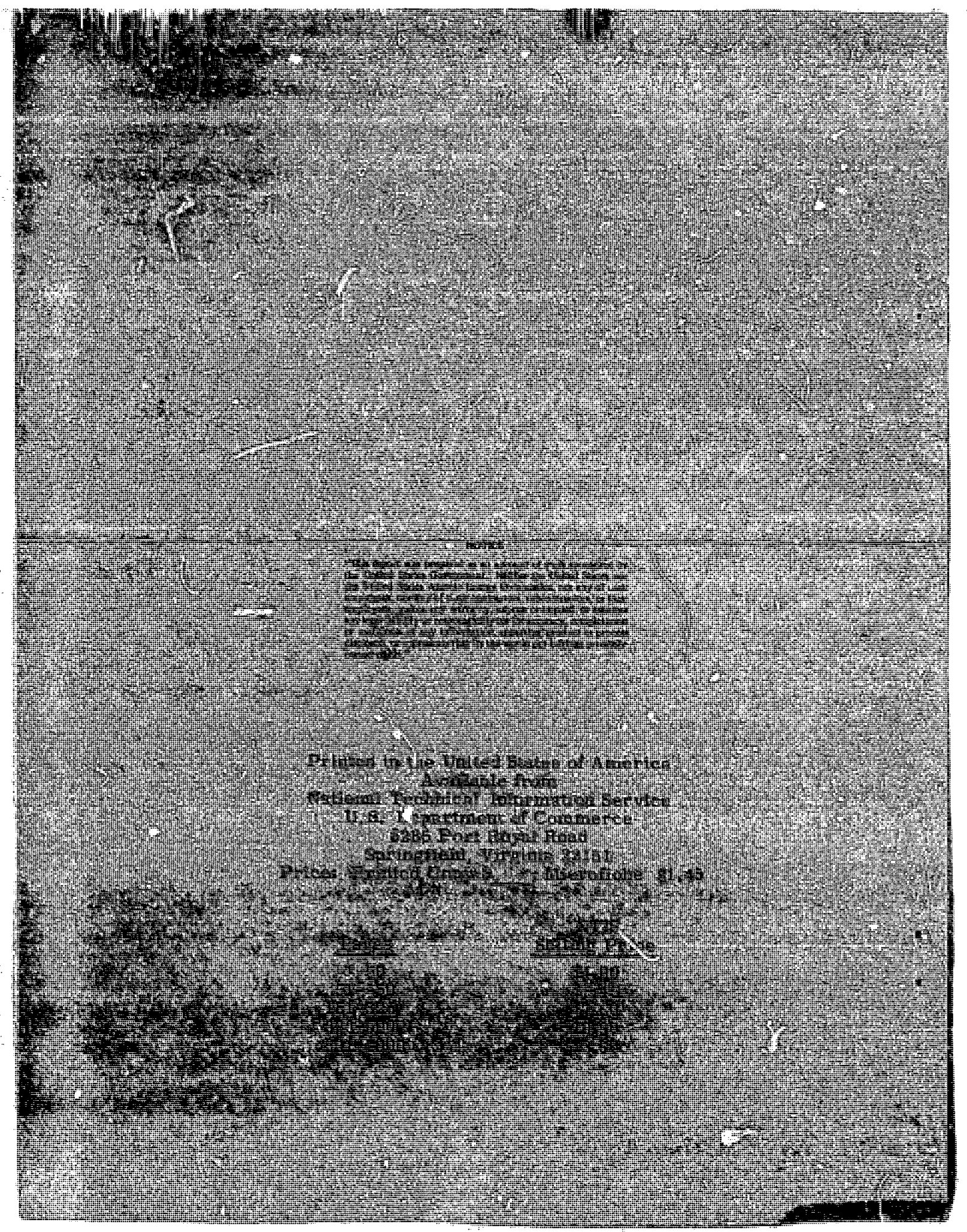





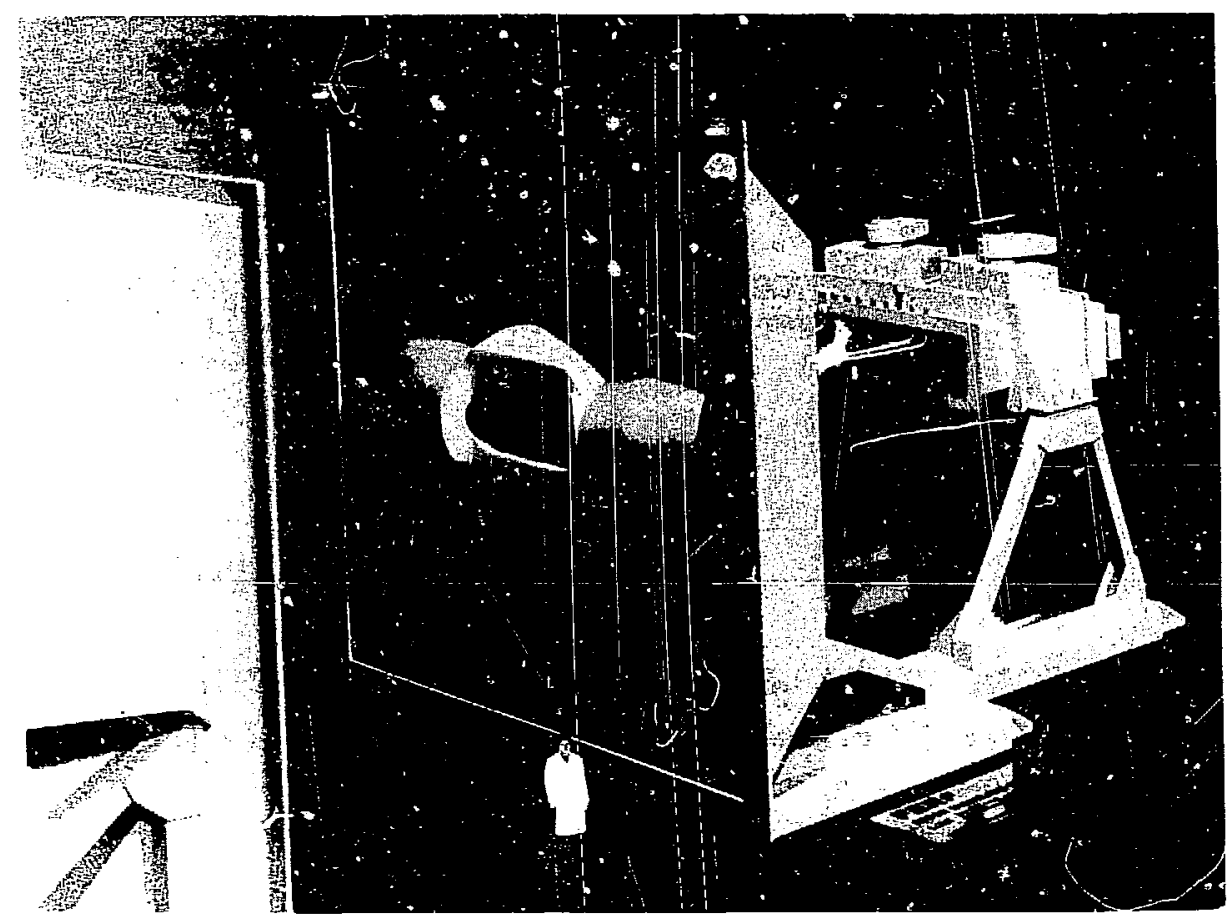

Frontispiece. Model of FERF reactor separated at midplane, showing su j irconducting magnet and support structure. 


\section{Contents}

Abstract

Rationale for a Fusion Energy Research Facility (FERF)

Plasma Physics Assumptions

General Description of Reactor and Facilities . . . . . . . . . . . . . . . . . . . . . . . . 4

Cost Estimate . . . . . . . . . . . . . . . . . . . . . . . . . . . . . . . . . . . 18

Construction Schedule. . . . . . . . . . . . . . . . . . . . . . . . 18

Safety . . . . . . . . . . . . . . . . . . . . . . . 21

References . . . . . . . . . . . . . . . . . . . . 21

Appendix A - Plasma Physics . . . . . . . . . . . . . . . . . . . . . . . . . . . 23

Plasma Parameters . . . . . . . . . . . . . . . . . . . . 23

Energy Scaling . . . . . . . . . . . . . . . . . . . . . . . . . . . . . . . . . . . 29

Magntetic Field Scaling. . . . . . . . . . . . . . . . . . . . . . . . . . . . . . 31

Plasma Stability . . . . . . . . . . . . . . . . . . . . . . . . . . . . . . . . 34

Adiabaticity . . . . . . . . . . . . . . . . . . . . . . . . . . . . . . . . . . . 34

Startup of the FERF PJasma $\ldots \ldots \ldots$. . . . . . . . . . . . . . . . . . . . . . 36

References . . . . . . . . . . . . . . . . . . . . . . 44

Appendix B - Neutral Beam Injectors. . . . . . . . . . . . . . . . . . . . . . . . . . 45

Selection of Injector Type . . . . . . . . . . . . . . . . . . . . . . . . . . . . 45

Injector Layout . . . . . . . . . . . . . . . . . . . . . . . . . . . . . . . 46 46

Gas Pumping Requirements. . . . . . . . . . . . . . . . . . . . . . . . 48

Plasma Source Modules . . . . . . . . . . . . . . . . . . . . . . . . . . . . . . . 49

Design Considerations: Acceleration-Deceleration Electrodes . . . . . . . . . . . . . . . 49

Source Reliability . . . . . . . . . . . . . . . . . . . . . . . . . . . . . . . . 53

Injector Power Supply Requirements . . . . . . . . . . . . . . . . . . . . . . . . . . 54

Neutral Beam Control Equipment . . . . . . . . . . . . . . . . . . . . . . . . 58

Long-Life Filament Design . . . . . . . . . . . . . . . . . . . . . . . . . . . . . . . 59

References ..................... . . . . . . . . .

Appendix $\mathrm{C}-$ Wall Bombardment by Neutral Atoms . . . . . . . . . . . . . . . . . . . 63

Introduction . . . . . . . . . . . . . . . . . . . . . 53

Analytic Model . . . . . . . . . . . . . . . . . . . . . 63

Mathematical Description . . . . . . . . . . . . . . . . . 64

Results of the Analysis . . . . . . . . . . . . . . . . . . . 65

Results for FERF . . . . . . . . . . . . . . . . . . . . . . . . . . . . . . . 69

References ......................... 73

Appendix $\mathbf{D}$-- Shield Analysis . . . . . . . . . . . . . . . . . . . . . . . . . . . . . 75

Introduction . . . . . . . . . . . . . . . . . . 75 
Culculational Techniques . . . . . . . . . . . . . . . . . . . . . . . . . 75

Geometrical Model . . . . . . . . . . . . . . . . . . . . . . . . . . . . . . . . 75

Materials Selected for Model . . . . . . . . . . . . . . . . . . . . . . . . . . . 78

Results of Analysis . . . . . . . . . . . . . . . . . . . . . . . . . . . . . . . . 78

Shut-Down Activities and Dose Rates . . . . . . . . . . . . . . . . . . . . . . . . . . 83

Conchusions . . . . . . . . . . . . . . . . . . . . . . . . . . . . . . . . . . . 85

Reterences... . . . . . . . . . . . . . . . . . . . . 86

Appendix E - Remote Assembly/Disussembly Procedures . . . . . . . . . . . . . . . . . . . 87

Objectives . . . . . . . . . . . . . . . . . . . . . . . . . . . . . . . . . . 87

General Requirements for Handling Systems . . . . . . . . . . . . . . . . . . . . . . . 87

Translationa! Movement Using Integral Dollies . . . . . . . . . . . . . . . . . . . . . . 87

Use of an Overhead Crane . . . . . . . . . . . . . . . . . . . . . . . . . . . . . . 88

Remote Positioning and Clamping . . . . . . . . . . . . . . . . . . . . . . . . . . . 89

Appendix F .- Safety Analysis. . . . . . . . . . . . . . . . . . . . . . . . . . . . . 93

Philosophy . . . . . . . . . . . . . . . . . . . . . . . . . . . . . 93

Source of Hazards . . . . . . . . . . . . . . . . . . . . . . . . . . . . 93

Tritium Use . . . . . . . . . . . . . . . . . . . . . . . . . . . . . . . . . 93

Radiation Levels . . . . . . . . . . . . . . . . . . . . . . . . . . . . . . . . 94

Accident Analysis . . . . . . . . . . . . . . . . . . . . . . . . . . . . . . . . 95

Applicable Regulations . . . . . . . . . . . . . . . . . . . . . . . . . . . . . . . 96

Environmental Assessment . . . . . . . . . . . . . . . . . . . . . . . . . . . . . . 97

Conclusions . . . . . . . . . . . . . . . . . . . . . . . . . . . . . . . . . . . . 97

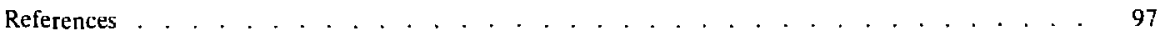

Appendix G-Tritium. . . . . . . . . . . . . . . . . . . . . . . . . . . . . 99

Introduction . . . . . . . . . . . . . . . . . . . . . . . . . . . . 99

Fuel Cycle . . . . . . . . . . . . . . . . . . . . . . . . . . . . . . . . . 99

Trilium in Magnet Shields . . . . . . . . . . . . . . . . . . . . . . . . . . . . . 102

Containment of Tritium . . . . . . . . . . . . . . . . . . . . . . . . . . . . . . . 103

Monitoring and Personnel Protection . . . . . . . . . . . . . . . . . . . . . . . . . 109

Conclusions . . . . . . . . . . . . . . . . . . . . . . . . . . . . . 110

References................................ 110

Appendix $\mathrm{H}$ - Magnet Design . . . . . . . . . . . . . . . . . . . . . . . . . . . $1 \mathbf{3}$

Winding Configuration . . . . . . . . . . . . . . . . . . . . . . . . . 113

Structural Design. . . . . . . . . . . . . . . . . . . . . . . . . . . . . . . . 114

Fabrication of Windings . . . . . . . . . . . . . . . . . . . . . . . . . . . . . . . 120

Magnet Power Supply . . . . . . . . . . . . . . . . . . . . . . . . . . . . . . 121

Appendix I - Vacuum Pumping Requirements . . . . . . . . . . . . . . . . . . . . . . 123 
Injector Pumping System. . . . . . . . . . . . . . . . . . . . . . . . . . . . . . . 123

Mirror Chamber Pumps . . . . . . . . . . . . . . . . . . . . . . . . . . . . . . 123

Recycling Pump . . . . . . . . . . . . . . . . . . . . . . . . . . . . . . . . . 124

Appendix J - Heat-Flux Capability of the First Wall . . . . . . . . . . . . . . . . . . . . . 125

Definition of the Problem . . . . . . . . . . . . . . . . . . . . . . . . 125

First-Wall Concept . . . . . . . . . . . . . . . . . . . . . . . . . . . . . . . . 125

Thermal Stress Limitation . . . . . . . . . . . . . . . . . . . . . . . . . . . . . 125

Tranjient Behavior . . . . . . . . . . . . . . . . . . . . . . . . . . . . . . . . 127

Pressure Drop and Pumping Power Constraints . . . . . . . . . . . . . . . . . . . . . . . 128

Design for FERF . . . . . . . . . . . . . . . . . . . . . . . . . . . . . . . . . 129

Critical Heat Flux . . . . . . . . . . . . . . . . . . . . . . . . . . . . . . . . . 130

Comparison of Materials . . . . . . . . . . . . . . . . . . . . . . . . . . . . . . 132

Effect of Parameter Variations . . . . . . . . . . . . . . . . . . . . . . . . . . . 132

Conclusions . . . . . . . . . . . . . . . . . . . . . . . . . . . . . . . . . . . . 132

Annex 1 - Equations Used in This Study . . . . . . . . . . . . . . . . . . . . . . . . 133

Anre* 2 - Method of Solution for the Maximuin Allowable Heat Flux . . . . . . . . . . . . . 135

Arinex 3 - Example Calculation . . . . . . . . . . . . . . . . . . . . . . . . . . . 136

References... . . . . . . . . . . . . . . . . . . . . . . . 138

Appendix K - Building Complex . . . . . . . . . . . . . . . . . . . . . . . . . . . . 139

Building Functions . . . . . . . . . . . . . . . . . . . . . . . . . . . . . 139

Descriptions of Principle Areas . . . . . . . . . . . . . . . . . . . . . . . . . . . . 139

Site Requirements . . . . . . . . . . . . . . . . . . . . . . . . . . . . . . . . 144

Appendik L - Details of Reactor and Reactor Facilities Costs . . . . . . . . . . . . . . . . . 145

Cost Estimating Methods . . . . . . . . . . . . . . . . . . . . . . . . 151 


\title{
CONCEPTUAL DESIGN OF A MIRROR REACTOR FOR A FUSION ENGINEERING RESEARCH FACILITY (FERF)
}

\begin{abstract}
A small non-power-producing mirror reactor has been desi,med for testing and evaluating the materials anc components which will be used in early fusion reactors. This small fusion reactor must produce a maximum useful 14-MeV neutron flux in a volume which is large enough to accommodate many engineering-scale test specimens, yet small enough to have reasonable cost. The required technology must be close to present practice, and the required extrapolation of plasma physics beyond present experimental knowledge must be minimal. The fusion

report produces about $1.1 \times 10^{14}$ neutrons $\cdot \mathrm{cm}^{-2} \cdot \mathrm{s}^{-1}$ at the edge of the plasma for the "low-field" coil (with $\mathrm{Nb}$-Ti superconductor) although about $3.4 \times 10^{14}$ neutrons $\cdot \mathrm{cm}^{-2-1,-1}$ would be possible in a "high-field" version (with $\mathrm{Nb}_{3} \mathrm{Sn}$ superconductor). The plasma radius is $25 \mathrm{~cm}$ at the center. With $13 \mathrm{MW}$ of injected neutral beam, a fusion power of $3.4 \mathrm{MW}$ is produced. The facility is capable of injecting $37 \mathrm{MW}$ of beam in case end losses are greater than predicted. A tritium processing and containment system for the facility has been designed.
\end{abstract} engineering research facility (FERF) described in this

\section{RATIONAT E FOR A FUSION ENGINEERING RESEARCH FACILITY (FERF)}

It is widely recognized that fusion reactors will create a very severe environment for construction materials. Just how severe is not known because there is little directly applicable experience. However, it is known that the neutron radiation damage in metals caused by each 14-MeV fusion neutron will be much greater than that caused by much lowerenergy fission neutrons. The $14-\mathrm{MeV}$ neutron fluxes anticipated at the first wall of conceptual fusion reactors range from $2 \times 10^{13}$ up to $8 \times 10^{13} \mathrm{n} / \mathrm{cm}^{2}$. At lower energies, up to $10^{15} \mathrm{n} / \mathrm{cm}^{2}$ are expected.

If $i t$ is assumed that the radiation damage leading to metal embrittlement is proportional to the displacements per atom, then the damage rates for a given metal in a fusion reactor spectrum can be compared to those in other energy spectra. For example, Logan, et al., ${ }^{1}$ estimate that 4.7 times more damage is produced in niobium by a $14-\mathrm{MeV}$ neutron than by the EBR-II spectrum. In a fusion reactor, the uncollided, I4-MeV neutron flux at the first wall is accompanied by scattered neutrens which have a wide energy range depending on the specific design of the neutron-absorbing blanket. For a typical Slanket, ${ }^{2}$ the total flux may be 4.3 times the uncollided 14-MeV flux from the plasma and the corresponding damage rate in niobium (per source neutron) is 10 times that expested (per incident neutron) in EBR-ll. Aлother comparison, using somewhat different calcula cion techniques and a different reactor-blarket model, ${ }^{3}$ predicts 22 times greater damage rate (per source reutron) for 316 stainless steel than would be expected (per incident neytron) in EBR-II. It is apparent that a first-wall, $14 \mathrm{MeV}$ neutron flux of $10^{14} \mathrm{n} / \mathrm{cm}^{2} \mathrm{~s}$ will result in a higher rate of damage than is possible in any existing or planned fission reactor. It is also possible that this damage may be of a different nature.

There are ways to simulate the neutron environment in a fision reactor. For example, neutrons for simulation experiments can he produced by bombarding a tritium target with $a$ deuteron beam from an accelerator. However, the intensity of such sources is limited by the ability of the target to carry away the heat deposited by the beam. The most intense source of this type now in existence, the rotating target neutron source (RTNS) at LLL ${ }^{4}$, produces about $4 \times 10^{12}$ $\pi / \mathrm{cm}^{2} \times$ at small santples. An improved version is now 
being developed which will provide a flux in excess of $10^{13} \mathrm{n} / \mathrm{cm}^{2} \cdot \mathrm{s}$. Other beam-target devices using deuterium gas targets are being considered for this purpose by the AEC's Division of Controlled Thermonuclear Research; however, al! such devices will have a very limited source size because of heat-dissipation problems. For example, a beam-target device yields about $3 \times 10^{9} 14-\mathrm{MeV}$ neutrons per joule of beam energy. Technical problems of hent dissipation would probably prohibit the operation of such a device because a spherical source with $1 \mathrm{~m}^{2}$ of surface area and a 14-MeV neutron current of $10^{14} \mathrm{n} / \mathrm{cm}^{2}$ 's would require $300 \mathrm{MW}$ of beam power. By contrast, a plasma-confinement device such as the FERF described in this report will produce the same results (assuming classical ion confinement) with a beam power of about $8 \mathrm{MW}$.

First-wall surfaces will also be bombarded with energetic ions and neutral atoms ranging up to several hundred $\mathrm{ke}^{\mathrm{N}}$ of $\mathrm{D}$ and $\mathrm{T}$, with $\mathrm{He}^{++}$from the DT reaction up to $3.5 \mathrm{MeV}$, and with intense electronagnetic radiation ranging up to $\sim 10-\mathrm{keV} x$ rays. Smallscale experiments using the focused beams of particle accelerators are being used to study several of these effects. However, it is difficult to predict the effects of simultaneous neutron and atom bombard nent: FERF wuld make these studies possible on a large scale.

Materials for use in fusion reactors should be tested as soon as possible using an actual reactor. The lesson lads been :"arned because of serious material problems (e.g., swelling of core materials) in fission power reactors that werr not ricognized until high powerdensity reactors were built. These problems were not observed in small-sample testing. In-pile testing started many years ago on several materials now being considered for use in fission reactors: radiation-damage effects should also be simulated on the engineering scale required for reliable design of large fusion-power plants.

In addition to irradiation of materials, FERF would serve as a proving ground for engineering a complete, integrated, tritium-handling system, large superconducting magnets, and high-power neutral-beam injector's. All of these will probably be essential parts of full-sicale fusion-power reactors of either the toroidal or mirror type.
On the pages that follow, we describe a small, nonpower producing, mirror reactor designed for use in the testing and evaluation of matzrials and components for use in early fusion reactors. FERF must produce a maximum useful 14-MeV neutron flux in a volume which is large enough to accommodate many engineering-scale test specimens, yet be small enough to have a reasonable cost. The required technology must be close to 1 ,resent practice, and the required extrapolation of plasma physics beyond present experimental knowledge must be minimal. The FERF design described here is not completely optimized, but it is believed to have near-optimum physics parameters.

The report is divided into two presentation modes. The first contains a summary of the study, and brings up-to-date the contents of an earlier, preliminary summary. ${ }^{5}$ Appendices contain detailed information on each aspect of the study, including the reactor and its components, auxiliary facilities and buildings, relevant physics, safety considerations, préiminary development required prio $i$ to construction, estimated costs, and construction schedule.

\section{PLASMA PHYSICS ASSUM?TIONS}

We have assumed that a mirror-confined plasma can be produced which satisfies plasma equilibrium and stability considerations. The design presented in this report comes very close to satisfying known stability conditions. (Future desigris will show a slightly modified coil shape which will stabilize interchange mooes by producing a greater radial magnetic weil depth.) The current design allows for an injected beam current about three times greater than would be required to sustain classical ion-ion collision losses. The degiee to which microinstabilities might affect these end losses is not well known. A major goal of present experimental and theoretical e[forts is to learn how these losses vary witl energy and other co.nfinement parameters. Because of the need for a maximum production of neutrons, parameters have been chosen to maximize neutron flux at the plasma surface. This leads to much lower energies and lower power gain than would exist in a power reactor design. The main plasma physics 
considerations detailed in Apperadix A may be summarized as follows:

$$
P=\frac{(\overline{\sigma v})}{4} T_{T}^{E_{f}} \int_{v} n^{2} d v,
$$

where $n=$ ion density (we have assumed $n_{T}=n_{D}=1 / 2 n$ ),

$$
\begin{gathered}
\mathrm{E}_{\mathrm{C}}=\text { fusion energy }=17.58 \mathrm{MeV} \text {, and } \\
(\bar{\sigma} \bar{v})_{\mathrm{DT}}=\begin{array}{l}
\text { reaction rate parameter avera } 5 \text { ed } \\
\text { over the ion velocity distribution. }
\end{array}
\end{gathered}
$$

Except for the small fraction of beam lost by chargeexchange processes, the injected beam current approximately equals the current of ions lost from the plasma by coulomb collisions; that is,

$$
I_{\text {loss }}=\frac{q}{n r} \int_{v} n^{2} d v
$$

where $\boldsymbol{\tau}=$ mean ion lifetime

To maximize fusion power, we must maximize $(\bar{\sigma} \bar{v})$ DT' $n^{2}$. For a fixed maximum field limited by superconductor technology, $\mathrm{n}^{2} \alpha 1 / \mathrm{T}^{2} \cdot(\overline{\sigma \mathrm{V}}) \mathrm{UT}$ also decreases with temperature below $100 \mathrm{keV}$, but much more slowly than with $\mathrm{T}^{2}$. Therefore, over a large temperature range the minimum plasma temperature will result in maximum fusion power and therefore, maxinum neutron flux. However, a practical minimum of about $65 \mathrm{keV}$ is dictated by practical limits placed on beam power, bcam power density, beam penetration ,f the plasma, and wall bombardment by charge-exchange neutrals.

The power gain, $Q$, of concern to reuctor designsrs is

$$
\mathrm{Q}=\frac{\mathrm{P}}{\mathrm{I}_{\text {loss }} \mathrm{W}_{\text {inj }}}=\frac{{ }^{(\bar{\sigma} \bar{v})} \mathrm{DT}^{(\mathrm{n} \tau) \mathrm{E}_{\mathrm{f}}}}{4 \mathrm{~W}_{\mathrm{inj}}},
$$

where $W_{\text {inj }}$ is injection energy.

For a vacuum mirror ratio of 2 , central vacuum magnetic field of $3.75 \mathrm{~T}$, injected ion energies of $65 \mathrm{keV}$ for $\mathrm{D}^{0}$ and $97.5 \mathrm{keV}$ for $\mathrm{T}^{0}$, and injection direction determined by gemetrical constraints. we can calculate the quantities which give nejtron produc tion rate and injector power requirements: using the following constants:

1. $\mathrm{n}$ is limited by the mirror instability which, for our parameters, is $\beta<0.65$ where $\beta=P / B_{0}^{2} / 2 u_{0}$ ) $\mathbf{P}=$ total plasma pressure perpendicular to the n.agnetic field $\mathrm{B}_{0}=$ vacuum central field.

2. End losses are determined from frokker-Planck computations, (both one-dimsnsional multispecies ano two-dimensional, single-ion species) give $n$, $(\widehat{\sigma o v})_{D T}$, and the exact relationship between $P$ and $n_{0}$ where $\mathrm{n}_{0}=$ central density.

3. The axial density profile is also determined from these Fokker-Planck calculations. The radial density profile is symretrical at midplane and is assuined to be cubic in r. This F.ofile compares well with calculated equilibrium profiles which account for nautral borm input and chargeexchange loss processes.

Thus, $\int \mathrm{n}^{2} \mathrm{dv}$ is determined and the resulting plasma parameters are summarized in Table 1 .

Three classes of instability are considered: Mirror modes, which limit $\beta$ to less than $\sim 0.65$ according to present theory :itterchange modes, which are suppressed by the sufficient de $\mathrm{e}_{\mathrm{i}}$ th of the radial magnetic well produced by the Yin-Yang coil; and microinstabilities, which could cause enhanced scattering losses. Our design allows for enhanced scattering by as muc'; as a factor of three over the classical rate. Recent theory and experimental results on 2 XIl support the expectations that scattering losses will be within this range. $A$ niajor goal of present mirror experiments is to verify the expected decrease of scattering losses with increasing temperature.

The size and strength of the FERF magnetic field is sufficient to contain the plasma adiabatically. A small nonadiabatic loss will occur fror $r_{i}$ the high-encrgy "tail" of the $\mathrm{T}^{+}$distribution. The $35-\mathrm{MeV} \mathrm{He}^{+}$ fusion-produted ions will be rapidly lost and will not contribute to plasma heating. (The $\mathrm{He}^{++}$would be contained in a device greater than about two times larger than FERF.

Startup will proceed from neutral beam uombardment of a small ( 908) target plasma. To provide sufficient target lifetime and buildup rate, this ta _et 
Table 1. Plasma Parameters (Low-Field Case).

$$
\begin{aligned}
& \text { Magnetic field intensities: } \\
& B_{\text {vac center }}=3.75 \mathrm{~T} \\
& B_{\text {center }}=2.22 \mathrm{~T} \\
& B_{\text {mirror }}=7.5 \mathrm{~T} \\
& B_{\text {conductor }}=9.0 \mathrm{~T} \\
& \beta=0.65
\end{aligned}
$$

Vacuum mirror ratio, $\left(R_{\text {vac }}\right)=2.0$

Actual mirror ratio $(\mathrm{R})=\mathbf{3 . 3 8}$

Injection energy $\left(W_{i n j}\right)=65-k e V D^{0}, 97.5-k e V ~ T^{0}$

(equal ion velocities for $D^{0}$ and $T^{0}$ )

Injection current $\left(\mathrm{I}_{\mathrm{inj}}\right)=36-4-\mathrm{A} \mathrm{D}^{0}, 136-\mathrm{A} \mathrm{T}^{0}=500 \mathrm{~A}$

$$
\left(I_{\text {clissical }}=136 \mathrm{~A}\right)
$$

Injection piwer $\left(P_{i n j}\right)=36.9 \times 10^{6} \mathrm{~W}$

Density at center $\left(n_{\text {center }}\right)=2.95 \times 10^{14} \mathrm{~cm}^{-3}$

$$
\left(\mathrm{n}_{\mathrm{D}}=\mathrm{n}_{\mathrm{T}}\right)
$$

Detiteron energy in plasma $\left(W_{D}\right)=73 \mathrm{keV}$

Triton mean energy in plasma $\left(W_{T}\right)=93 \mathrm{keV}$

Electron mean energy in plasma $\left(W_{e}\right)=7.2 \mathrm{keV}$

Plasma radius $(r)=25 \mathrm{~cm}$ at midplane

Plasma length $(\mathrm{L})=420 \mathrm{~cm}$ between mirrors

Fusion power density $\left(\mathrm{P}_{0} / \mathrm{V}\right)=60 \mathrm{~W} \cdot \mathrm{cm}^{-3}$

Total fusion power $(\mathrm{P})=3.38 \times 10^{6} \mathrm{~W}$

Fusion power/injected trapped power capability of this design $(Q)=0.11$ (based on $17.58 \mathrm{MeV}$ per fusion event) the corresponding $n t=1.9 \times 10^{12}$ $\mathrm{cm}^{-3} \cdot \mathrm{s}$

Fusion power/injected trapped power for classical end-loss process $\left(\mathrm{q}_{\text {classical }}\right)=0.33$ (based on $17.58 \mathrm{MeV}$ per fusion event) the corresponding $n r_{\text {Elassical }}=5.8 \times 10^{12} \mathrm{~cm}^{-3} \cdot \mathrm{s}$, where $\tau=$ ion confinement time

lon gyroradii at plasma center:

$$
\begin{aligned}
a & =2.45 \mathrm{~cm} \text { for } 75-\mathrm{keV} \mathrm{D}^{+} \\
a & =3.39 \mathrm{~cm} \text { for } 93-\mathrm{keV} \mathrm{T}^{+} \\
\mathrm{a} & =12.0 \mathrm{~cm} \text { for } 3.52 \mathrm{MeV} \mathrm{H}^{++} \\
\mathrm{r}_{\mathrm{p}} / \mathrm{a} \mathrm{D}^{+} & =10.2
\end{aligned}
$$

$\mathrm{L} / \mathrm{a}=171$

$n / B^{2}=6.0$, where $n$ is in units of $10^{13} \mathrm{~cm}^{-3}$ and $B$ is in $10^{4} \mathrm{G}$ plasma must have approximately the following characteristics: density of $2 \times 10^{13} / \mathrm{cm}^{3}, \tau=100 \mu \mathrm{s}$ $\left(\mathrm{n \tau}=2 \times 10^{9}\right)$, and ion temperature of $\sim 1 \mathrm{keV}$. This plasma is similar to that already obtained in 2 XII. It can probably be created by several techniques, one of which is pellet heating using a $1-\mathrm{kJ} \mathrm{CO}_{2}$ laser.

During buildup and also during steady-state, the outer surface of the plasma is continuously bombarded by neutral gas from two sources: some streams through the neutral beam injection ports; and some results from the diffusion of DT gas out of the first wall where it is continuously being implanted as fast neutrals from charge-exchange reactions.

A calculation model of the buildup assumes that the plasma is a nest of concentric shells of finite thickness and constant density and includes the following effects: mixing effect of finite Larmor orbits, radial diffusion, scattering into the nirror-loss region of velocity space, heam attenuation, and charge exchange. Results show that the plasma can withstand these erosion effects; however, the ability of the FERF low-energy plasma to "burri-out" incoming gas is near the limit.

The low energy chosen for this plasma to maximize fusion power density results in a high flux of neutral atoms from charge exchange between the beam and the plasma ions. This effect is discussed in more detail in Appendix $\mathrm{C}$.

About $5.9 \mathrm{MW}$ of $\mathrm{D}^{0}$ and $\mathrm{T}^{0}$ reach the first wall. This results in ion-uniform distributions of both heat deposition and sputtering. The maximum flux is $7 \times 10^{16}$ atoms $/ \mathrm{cm}^{2}-5$, which corresponds to a heat flux of $\sim 800 \mathrm{~W} / \mathrm{cm}^{2}$. The first wall must alsu occasionally withstand the neutral beam bombardment for short periods of time during startup or when the plasma is extinguished. The way the first wall is designed to withstand this bombardment is discussed briefly on page 6 and in more detail in Appendix $J$.

\section{GENERAL DESCRIPTION OF REACTOR AND FACILITIES}

Figure I shows a general layout of the reactor with the principle components identified. In the following pages, we will discuss lhe major components of the 


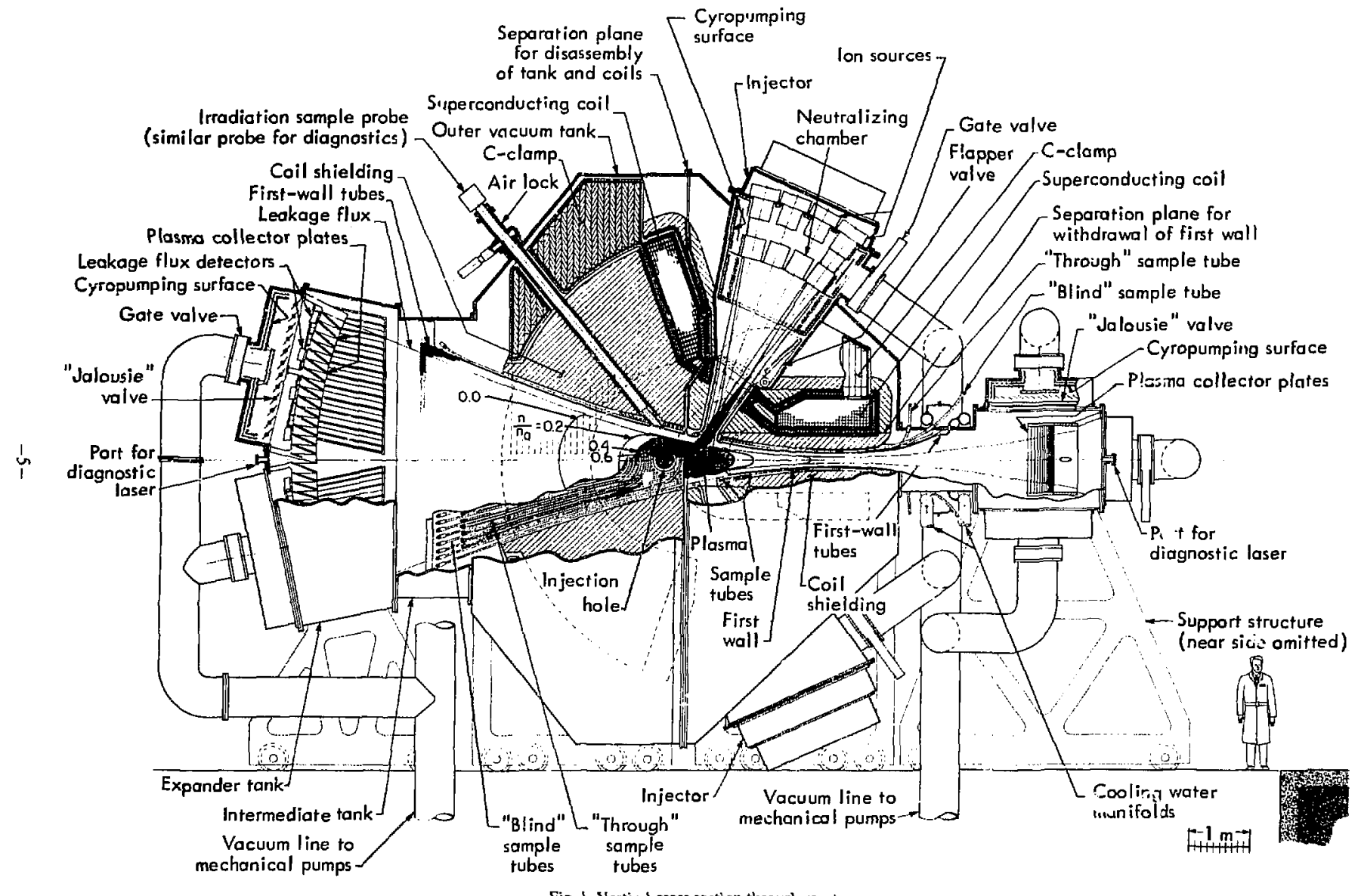

Fig. I. Vertical cross section through reactor. 
reactor, including the superconducting coils, coil support structure, first wall, facilities for sample irradiation, shielding. neutral beam injectors, plasma collector plates, outer tank, maintenance facilities, reactor building complex, and tritium system.

\section{Superconducting Coil System}

The Yin-Yang coil shape (Fig. 2) is one of the most efficient in terms of providing a hydromagnetically stable magnetic well with a reasonable mirror ratio. It also provides a natural beam injectio access between the two coils and facilitates the assembly and removal of the first wall. The conductor musi be rather carefully distributed to minimize the magnetic field at the superconductor. Details of magnetic design, structural design, and electrical design are given in Appendix $\mathrm{H}$.

For the low-field case of a 9.0-T maximum field at the conductor, $\mathrm{Nb}-\mathrm{Ti}$ superconducting material can be used if it is refrigerated to about $3.5 \mathrm{~K}$; also, a dual conductor system using $\mathrm{Nb}_{3} \mathrm{Sn}$ in the higher-field regions is probably practical. For the high-field case of 12.0-T maximum, at least one third of the conductor must be $\mathrm{Nb}_{3} \mathrm{Sn}$ or equivalent high-field material. A multifilamentary $\mathrm{Nb}_{3} \mathrm{Sn}$ material, which promises to be more practical than present-day thin tape, is under active development and would probably be used throughout the entire coil. A detailed design of the conductors has not been made; however, enough space is allowed for an overall current density of $2700 \mathrm{~A} / \mathrm{cm}^{2}$ (for the $\mathrm{Nb}$-Ti case). A preliminary structural design shows that support of the enormous magnetic forces is possible for both the low-and high-field cases using the structural system shown in Fig. 3. As shown in Fig. 4, the windings are enclosed in a massive, stainless-steel-coil tank. This coil tank is in turn enclosed in a thermal shield consisting of a nitrogencooled copper plate and a multilayered reflective heat shield. The coils, coil structure, and multilayer insulation are contained in the main tank, shown in Fig. 5 , vll :h is evacuated to minimize heat leak .

\section{Coil-Support Structure}

Enormous magnetic forces are produced by the coils. One component of these forces tends to straighten out the arc of the coil into a straight line.
This force can be resisted by the coil tank itself. Another component of these forces tends to open up the coil gap. Because it is impractical to make the coil tank strong enough in itself, $m$ wsive $C$-clamps (Fig. 3) are provided to resist this force. These Cclamps are fabricated of stainless steel plates bolted together. For convenience in handling, each C-clamp may be divided into several subassemblies.

There is also a large force urging the two coils toward each other. This force is resisted by a doublewalled, conically shaped, intercoil structure (see Fig. 3), which is pierced by holes for the four injectors and by holes for probes.

Both the C-clamps and the structure between the coils are cooled to the same temperature as the coils. Therefore, these members must also be surrounded by the same type of thermal shield as the coil. This arrangement eliminates the problems related to differantial thermal contracticn and the transmission of large forces across thermal barriers.

Coil parameters are given in Table 2 . For the lowfield' case, the conductor weight is $7.3 \times 10^{4} \mathrm{~kg}$, and the weight of the coil case and structure is $8.6 \times 10^{5} \mathrm{~kg}$. Th: total heat input is $10 \mathrm{~kW}: 6.3 \mathrm{~kW}$ from radiation herating $\mathrm{i}$ the coil and structure, $2.5 \mathrm{~kW}$ from heating in join $\mathrm{s}, 800 \mathrm{~W}$ from thermal radiation and conduction through supports. and $400 \mathrm{~W}$ from transfer lines and current leads. About 3 weeks will be required for cool-down.

Firs: Wa!l

The purpose of this first wall is

- To stop the high-energy particles which escape from the plasma across the magnetic field and to remove the heat deposited by these particles. This flux consists primarily of $D^{0}$ and $T^{0}$ from charge exchange between injected beam and plasma and $\mathrm{He}^{++}$from DT fusion reactions.

- To provide a high-vacuum environment for the plasma.

- To contain tritium within a prescribed volume.

- To withstand (occasionally) neutral beam bombardment for short periods of time during startup or whon the plasma is exiinguished. 


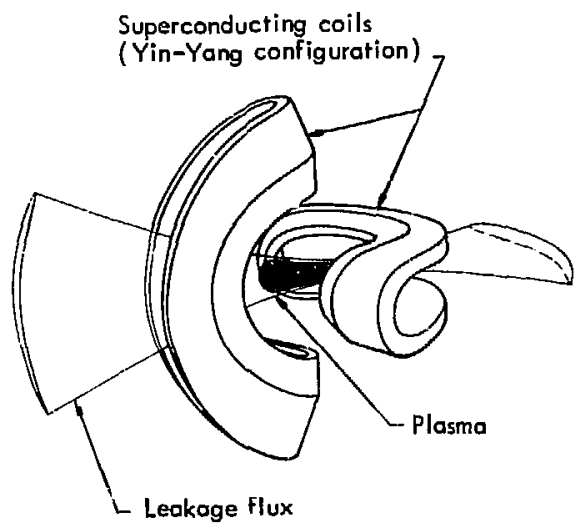

Fig. 2. Perspective view of coils and plasma.

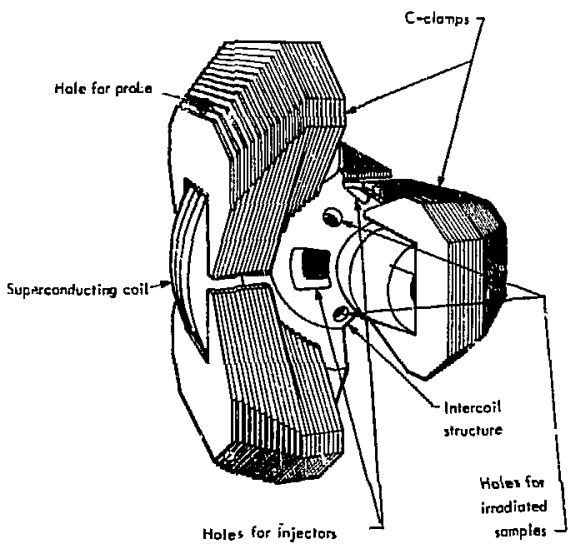

Fig. 3. Perspective view of coil support structure.

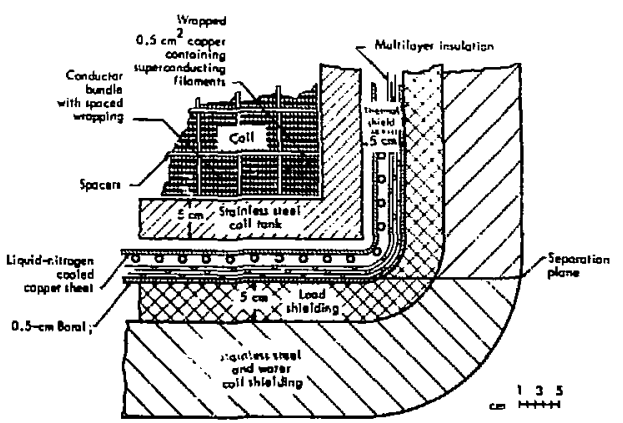

Fig. 4. Cross section of coil and shielding.

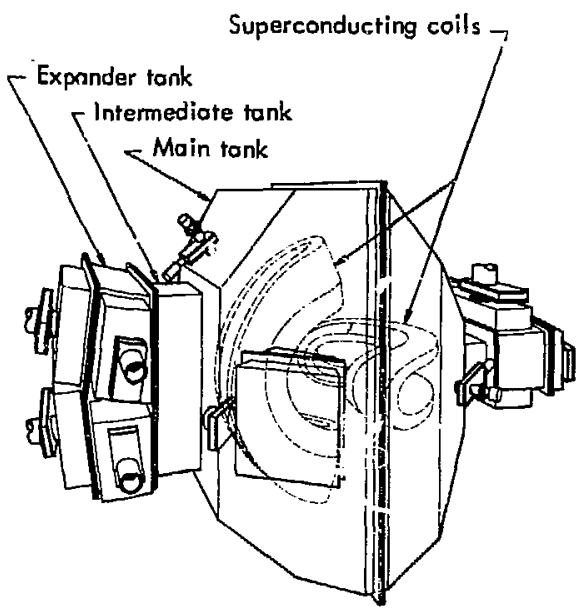

Fig. 5. Perspective view of coils in tank.

Thermal stresses are important and a comparison of severai wall materials sliows that refractory metals such as Mo-0.5\% $\mathrm{T}$ and Ta-10\% W are desirable, although an aluminum alloy such as 6061 may also be possible. The first-wall heat-transfer and thermalstress problem is discussed in considerable detail in Appendix J.

The first wall consists of two layers (see Fig. 6). The inner layer bears the brunt of the heat flux and the outer layer provides a vacuum-tight barrier to prevent tritium contamination of the coils, coil structure, and sample tubes. Both layers carry cooling water.

The, inner layer of the first wall, the layer next to the plasma, consists of a close-packed array of small ( 1-cm diameter), thin-walled tubes. The heat flux is, of course, highest near the center of the machine; therefore, the tubes here are small in diameter and the velocity of the water is necessarily very high. Away from the center, the heat flux is lower; therefore, the diameter of the tubes may be increased and the velocity of the water and consequently the required pressure and pumping power thereby reduced. As the perimeter of the first wall increases with distance from the center, the increase in tuhe diameter can be accommodated while the tubes are still kept in one layer. Because of the thermal stresses involved, it appears necessary to keep these tubes adjacent but 
Table 2. Coil parameters.

\begin{tabular}{|c|c|c|}
\hline & $\mathrm{Nb}-\mathrm{Ti}$ & $\mathrm{Nb}_{3} \mathrm{Sn}$ \\
\hline Central field $(T)$ & 3.7 & 5.0 \\
\hline Mirror field $(T)$ & 7.5 & 10.0 \\
\hline $\begin{array}{l}\text { Max. field at } \\
\text { conductor }(T)\end{array}$ & 9.0 & 12.0 \\
\hline Ampere turns & $17.6 \times 10^{6}$ & $23.5 \times 10^{6}$ \\
\hline Ampere-meters & $5.6 \times 10^{8}$ & $7.5 \times 10^{8}$ \\
\hline $\begin{array}{l}\text { Current density } \\
\left(\mathrm{kA} / \mathrm{cm}^{2}\right)\end{array}$ & 2.7 & 3.6 \\
\hline $\begin{array}{l}\text { Total force between } \\
\text { coils }\left(F_{z}\right)\left(10^{6} \mathrm{lb}\right)\end{array}$ & 11.3 & 21.2 \\
\hline $\begin{array}{l}\text { Total force normal to } \\
\text { flat sections } \\
\left(F_{\mathrm{V}}\right)\left(10^{6} \mathrm{lb}\right)\end{array}$ & $\sim 120$ & $\sim 210$ \\
\hline$F_{x}\left(10^{6} l b\right)$ & 44 & 78 \\
\hline $\begin{array}{l}\text { Minimum conductor } \\
\text { radius }(\mathrm{cm})\end{array}$ & 55 & 55 \\
\hline $\begin{array}{l}\text { Minimum radius } \\
\text { from reactor center } \\
\text { to conductor }(\mathrm{cm})\end{array}$ & 153 & 153 \\
\hline $\begin{array}{l}\text { Maximum distance } \\
\text { from reactor center } \\
\text { to conductor (cm) }\end{array}$ & 370 & 370 \\
\hline $\begin{array}{l}\text { Distance between } \\
\text { mirrors }(\mathrm{cm})\end{array}$ & 500 & 500 \\
\hline Stored energy $\left(10^{9} \mathrm{~J}\right)$ & 1.4 & 2.5 \\
\hline
\end{tabular}

not continuously attached. Therefore, a vacuumtight tritium barrier need not be provided by this layer alone. The outer layer of the first wall is made of extrusions welded together lengthwise to provide a vacuum barrier. These extrusions have integral passuges for cooling water.

Because of the extremely severe conditions to which the first wall is subjected, it will be periodically replaced. (In this sense, the first wall can be regarded as a very large irradiation sample.) However, because of its shape the first wall cannot be inserted into or withdrawn from the coils in one piece. The first wall is therefore split on the center line. The joint between the two halves would be very difficult to make up or take apart. This joint is therefore, in effect, carried right out to the joint between the two halves of the outer tank by means of two sheet-metal diaphragms. When the two halves of the outer tank

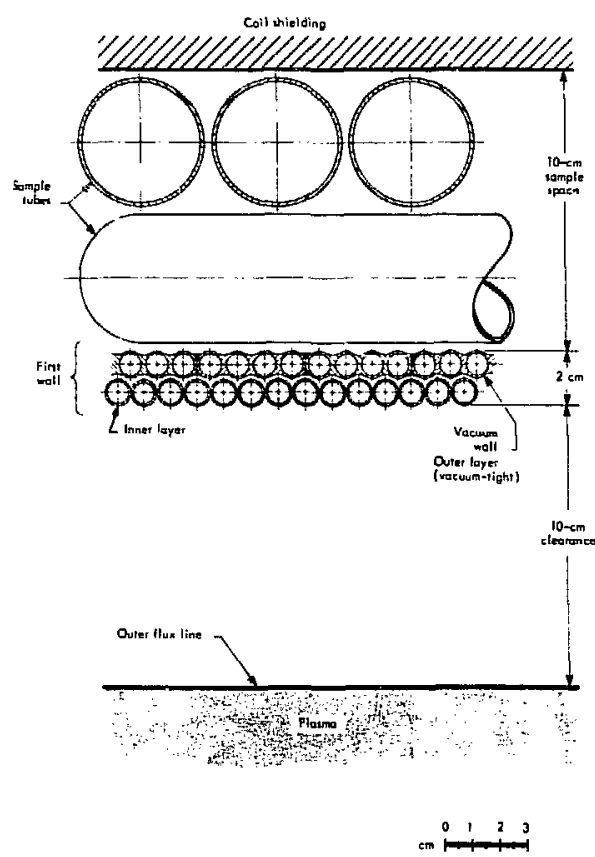

Fig. 6. Relationship of plasma, first wall, sample tubes, and coil shielding at ceruter.

(and the coil and first wall contained therein) are pulled apart, the joint between the first wall and the diaphragm becomes accessible. Each half of the first wall may then be withdrawn through its respective coil gap. The sample tubes and a portion of the coil shielding must also be withdrawn at the same time as one subassembly. This subassembly is cuntilevered from a section of tank intermediate betweer the outer tank proper and the expander tank. This intermediate tank also carries all the connections to tine sample tubes and the first-wall tubes.

\section{Facilities for Sample Irradiation}

\section{Radial Probes}

It must be possible to expose samples to the plasma without an intervening first wall. As noted above, the first wall itself may be regarded as a sample. In addition, radiation sample probes are provided which pierce the first wall. One such probe, shown in Fig. 7, penetrates the C-clamps and enters the plasma zone through 
the gap between the coils. The probe is a tube with a detachable sample at the end. The tube contains cooling lines and instrument leads. The probe may be withdrawn through an airlock without losing vacuum in the outer tank. An outer tube around the probe provides an inner vacuum sheath continuous with the first wall. Similar probes (not shown in the drawing) may be inserted into the machine between the two coils in a manner similar to that shown for the injectors. These latter probes would be positioned between the four injectors and would enter the inner zones through holes in the structure separating the two coils (see

Fig. 3).

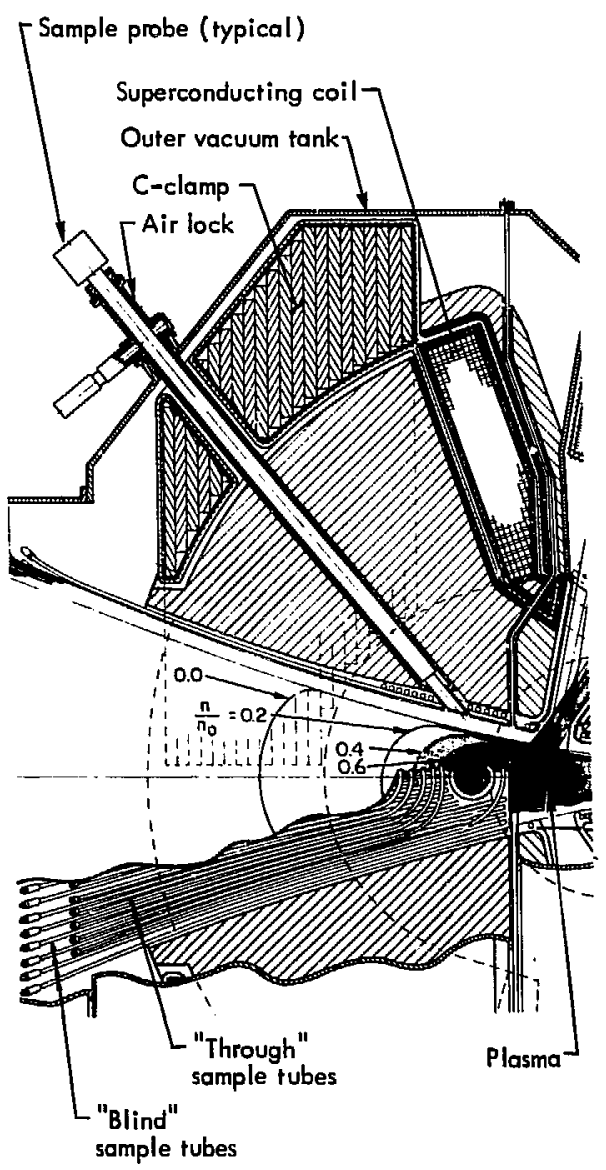

Fig. 7. Detail from Fig. I showing typical sample probe penetrating coil, shielding, and first wall.

\section{Tubes}

In addition to radial probes which penetrate the shielding and which may accommodate more complex instrumentation and coolant circulation, the entire volume between the vacuum wall and coils can be used for experimental access. Enough shielding must be retained to protect the coil. The design presented here has an experimental volume surrounding the plasma of about $1 \mathrm{~m}^{2}$ by about $10-\mathrm{cm}$ thick. This region can be modified to accommodate special requirements; however, for this study we have assumed that this space is nearly filled with a general-purpose array of about 60 5-cm-diameter sample tubes (Fig. 6). Samples to be irradiated can be inserted at the ends of the tubes (which may extend into a shielded area) and then positioned near the plasma region.

Two types of sample tubes are provided:

- "Blind" tubes provide straightforward insertion and removal of samples. As shown in Fig. 8, each tube consists of an inner and outer tube to provide coolant passages. A number of samples may be linked together and withdrawn by a suitable rod. Because these tubes terminate inside the biological shielding, appropriate manipulators must be used to load and unload them.

- "Through" tubes, shown in Fig. 9, are also immcdiately adjacent to the first wall. They are loaded in a manufacturing assembly area and unloaded in a disassembly and hot-cell area located outside the biological shielding. Thus, they have the advantage of being loaded and unloaded while the reactor is in operation. However, as the tubes unavoidably make a number of bends, sample carriers must have rolling contact. These sample tarriers are strung like beads on a continuous cable (Fig. 8).

\section{Shielding}

The radiation shield protects the superconducting coils from excessive heat input and from radiation damage. At the same time, the shield must be as thin as possible so that the magnet size and cost can be 

shield is approximately twice that of a 100 vol\% stainless steel shield but is still low enough to satisfy radiation damage criteria.

The neutron flux in the coil has an energy distribution approximately as shown in Fig. 10. Based on rather preliminary data on deterioration of superconducting properties in a "fast" neutron flux $(\mathrm{E} \approx 1 \mathrm{MeV}$ ), the shielding should be adequate. However, to properly select a shield design, more extensive datu obtained under realistic operating conditions are needed on damage by high-energy neutrons.

Calculations were performed with an LLL version of the MORSE Monte Carlo Code using a 45-group, coupled, neutron-gamma cross-section set. Results for cases where the maximum uncollided neutron flux density in the test region was equal to $10^{14} \mathrm{n} / \mathrm{cm}^{2}$ s indicated the total energy deposited in the magnets and their steel covers to be $5.2 \mathrm{~kW}(0.011 \mathrm{MeV}$ per source neutron). For the same case, $1.17 \mathrm{MW}$ was deposited in the test region and $6.44 \mathrm{MW}$ in the shield.

Shutdown dose rates and radioisotope activities throughout the system due to neutron interactions were calculated using coupled neutron-gamma crosssection sets in which the coupling $(n, \gamma)$ cross sections represented the activation cross section. A timedependent correction is then applied to the results to account for the reactor's operating history and the time shutdown. After one year's operation, the test

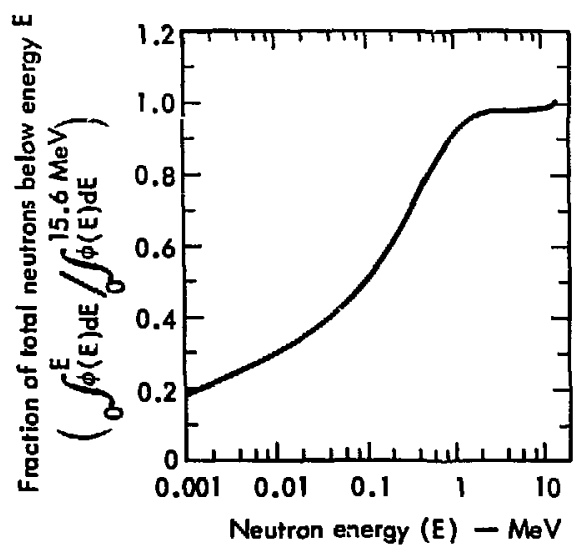

Fig. 10. One-dimensional calculations of the neutron spectrum in the magnet. region contains $176 \mathrm{Ci}$ of gamma emitters with half lives greater than 50 . The shield contains $4.7 \times 10^{6}$ $\mathrm{Ci}$, the lead $90 \mathrm{Ci}$, the magnet case $25 \mathrm{Ci}$, and the magnet itself $\mathbf{I} \mathrm{C}$. If no additional shielding is provided to prevent radiation from leaking out of the reactor, the dose rates outside the system could be as high as $12,000 \mathrm{rem} / \mathrm{h}$ immediately after shutdown and up to $100 \mathrm{rem} / \mathrm{h} 30$ days after shutdown.

From a shielding point of view, the conceptual FERF design seems to be a workable design, and the required shield characteristics can be achieved within the system limitations.

\section{Neutral Beam Injectors}

The injection system is described in Appendix B and is capable of a nominal $500 \mathrm{~A}, 360 \mathrm{~A}$ of $65 \mathrm{keV}$ $\mathrm{D}^{0}$ and $140 \mathrm{~A}$ of $100-\mathrm{keV} \mathrm{T}^{0}$; this is over three times the current required to sustain classical mirror losses and thus represents some degree of allowance for possible enhancement of mirror losses ahove the "classical" calculated rate.

The beam power density selected, $4 \mathrm{~kW} / \mathrm{cm}^{2}$, has been achieved in present sources; however, beam access is restricted by the magnet windings, magnet support structure, and shielding. Therefore, an array of converging beams is used to pass through the $20 \times 20 \mathrm{~cm}$ reactor-beam access holes. There are four beam lines with a $2 \times 2 \mathrm{~m}$ array of source modules located about $4 \mathrm{~m}$ from the plasma (as shown in Fig. 11). Positive inns are extracted from a multiaperture arc chamber and neutralized by $D_{2}$ and $T_{2}$ gas flowing from the source modules through ducts of appropriate length. See Fig. 12 for an example (scaled from an existing LLL source) of an arc chamber and extraction electrodes. Figure 13 shows a conceptual layout of the neutralizer and columnator arrangement.

The large amount of neutral ges, about $3200 \mathrm{~A}$, produced by the injectors is adequately pumped in two stages by cryopump panels which allow about $10 \mathrm{~A}$ to stream directly into the plasma. A nitrogencooled panel and multiple heat shields are provided outside the cryopumping surface to minimize the heat loss, as shown in Fig. 14.

The cryopumping surface must be periodically degassed. To perform this 2- to 3-minute degassing cycle, the ion sources are turned off and the flapper valve 


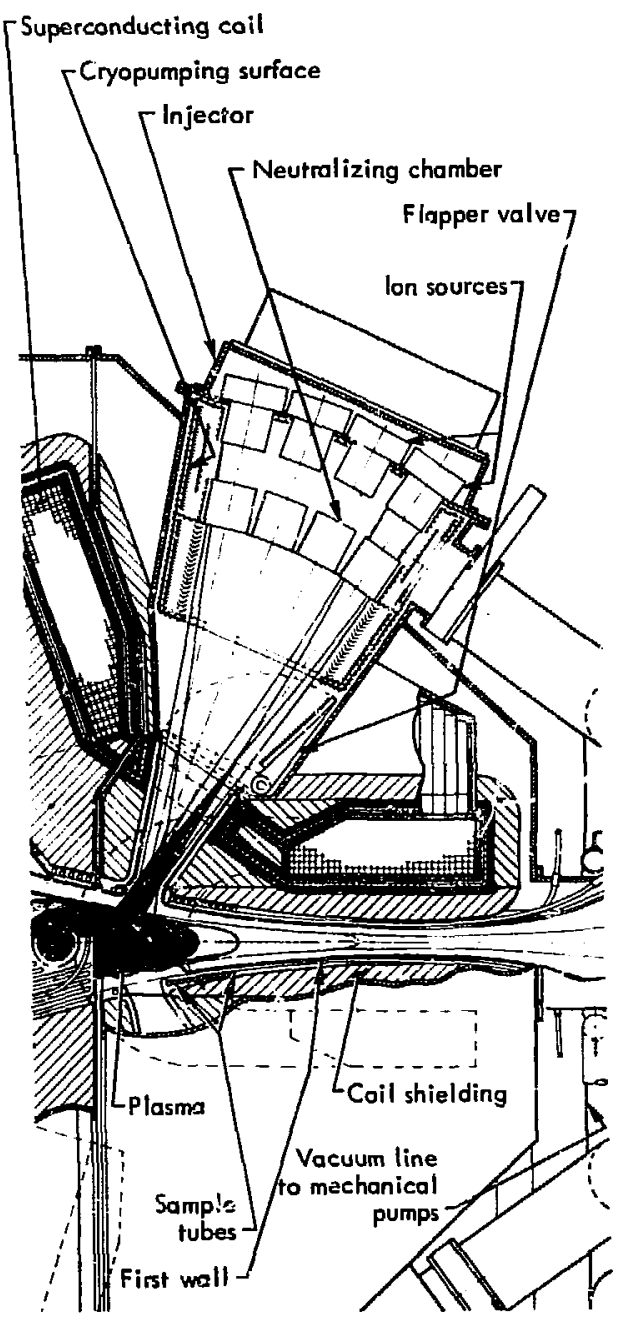

Fig. 11. Detail from Fig. I showing typical seclion of one of the injector points.

near the inner end of the injector chamber is closed. (Because the reactor is designed to run on three injectors, any one injector may be tunied off while the reactor is running.) The gate valve at the outer end of the chamber is opened to the line to the mechanical purnps, and the cryopumpirig surface is allowed to warm up to drive off the condensed gas. When this is completed, the gate valve is closed, the cryopumping surfaces cooled, the flapper valve opened, and the ion sources turned back on. This process is automatically seguerced for all four injectors. Pumping calcul t tions, are given in Appendix I.

The ion sources must also be periodically replaced. To do this, the flapper valve is clused to maintain the main vacuum and to minimize the escape of tritium into the vault. The sources are handled directly by the crane or by dollies and crane.

Each iniector assembly consists of 28 source modules and accompanying neutralizing chambers. The module desigr was obtained by scaling the performance of existing LLL 50-A, 20-keV pulsed scurces and allowing for appropriate de-rating for continuous operation. The total injector power required, including about $25 \mathrm{MW}$ of arc and filament power, is $122 \mathrm{MW}$.

\section{Plasma Collector Plat:}

The leakage flux from the plasma expands out:vardly along the magnetic field lines and is collected on plasma collector plates. These are simply watercooled target plates. In a power-producing reactor, the energy carried by this leaking plasma would be recovered at high ef ficiency by utilizing electrostatic deceleration in a "direcl converter". 6 The added cost and complexity of a direct converter is not justified for FERF because of the small amount of power to be recovered approximately 13 to $37 \mathrm{MW}$ ). However, the feasibility of a simple, direct converter for a FERF is being investigated in a separate study.

The louvered geometry of the plasma collector plates is advantageous because it presents an extended area to the leakage flux and thus reduces the lieating problems. At the same time, it presents a surface which is transparent to the large amount of gas which is produced when the charged particles are neutralized. Cryopumping is also used i ? this region. In this case, the cryopumping chambers are isolated for defrosting by "jalousie" valves as shown in Fig. 1 . These valves simply consist of a number of rotatable shutters. In contradistinction to the flapper valves on the injectors, the jalousie valves do not need to be vacuum tight becatse this chamber is always under vacuum.

Figure 15 shows details of the plasma collector plates. Thie front face of the plate, which sees the incorring leakage flux, is water-cooled to remove the 


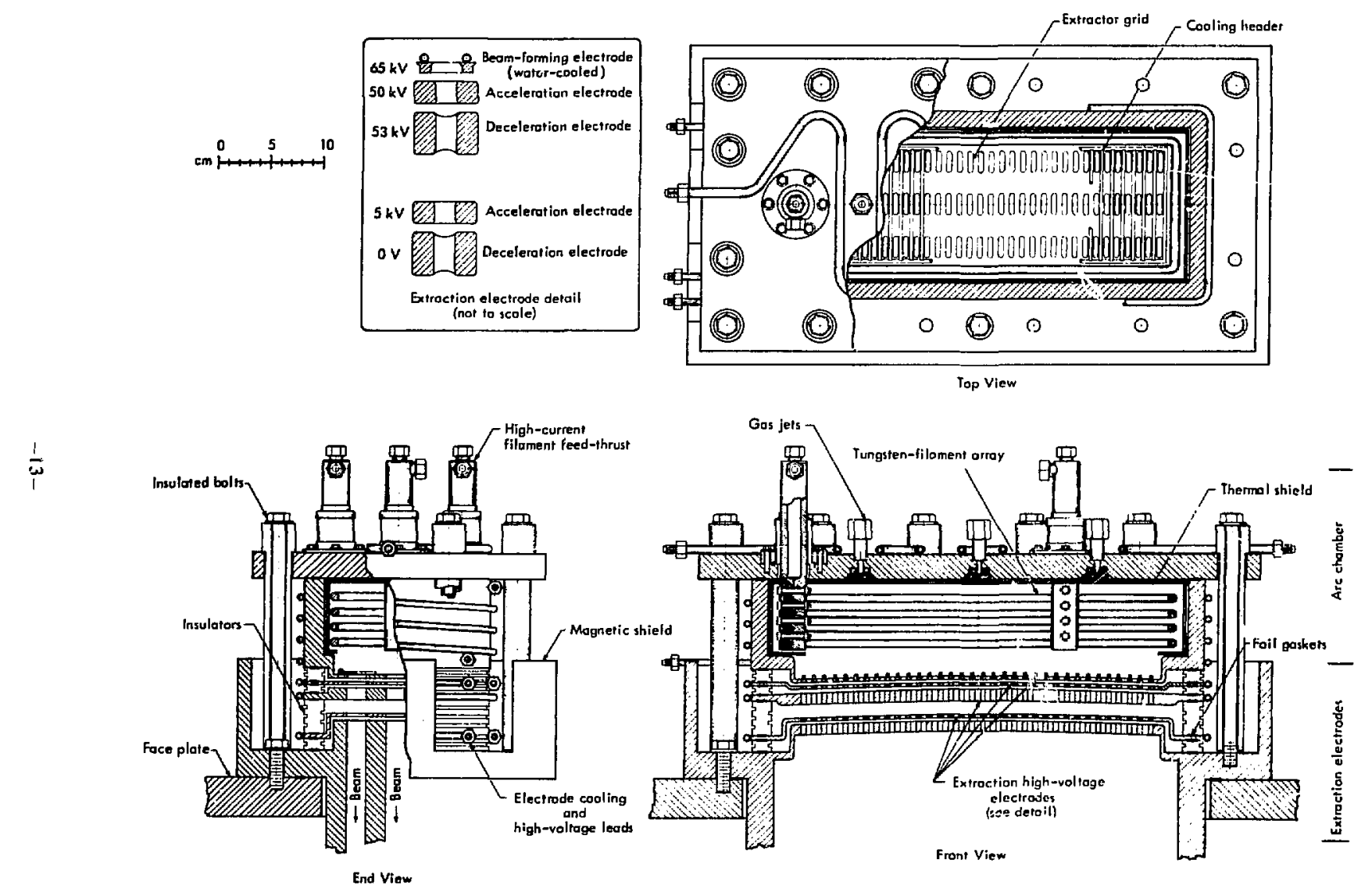

Fig. 12. Arrangement of ion source and neutralizing chamber. 

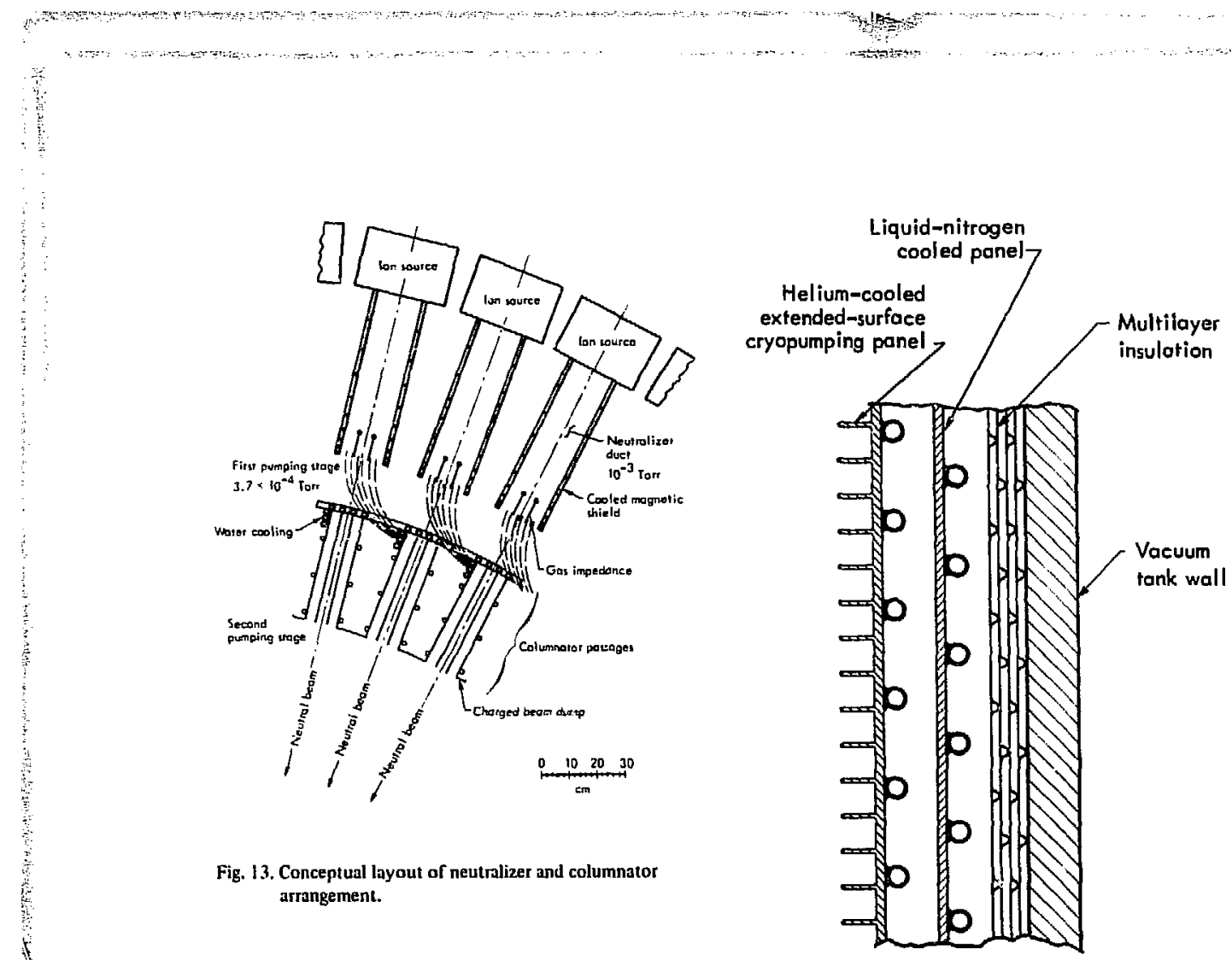

heat. The back is nitrogen-cooled to reduce the heat lond on the cryopumping surfaces. A multilayered reflective heat shield is placed between the watercooled front face and the nitrogen-cooled back face to reduce heat transfer between the two.

The vacuum lines from all the cryopumping chambers, both for the injectc - and plasma collector plates, are manifolded together into one line tu the mechanical pumps. Because only one cryopumping chamber will be difrosted at a time, this common line need be no larger than that required for one chamber. Cryopumping calculations for the plasma collector plates are shown in Appendix 1.

\section{Outer Tank}

Although the first wall is a vacuum bartier, because of its shape and necessarily flimsy construction it cannot support atmospheric loading. The outer tank, shown in Fig. 5, provides a vacuum outside the first wall and thus removes the pressure loading on the (irst wall. The outer tank also provides the insulating vacuum for the superconducting coils.

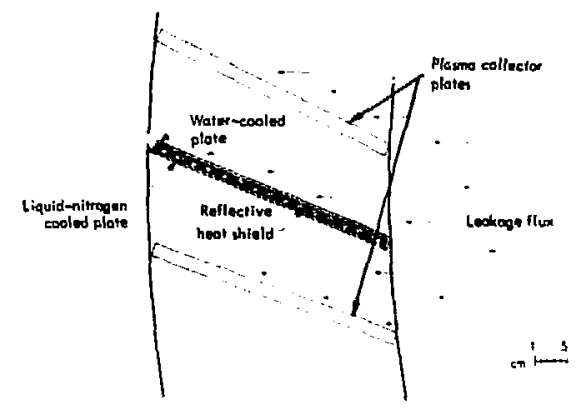

Fig. 15. Detail of plasma collector plate. 


\section{Maintenance Facilities}

Immediately after shutdown following 1 year of continuous operation, the dose rate at a representative location in the reactor room is $400 \mathrm{rem} / \mathrm{h}$, decreasing iv $100 \mathrm{rem} / \mathrm{h}$ in 30 days (see Appendix D). Disussembly anu' maintenance operations will, of course, require special equipmeni, manipulators, movable shields, and tools. An operator entering the vault must be enclosed in, say, af few inches of lead. This is true not only for major disassembly, but also for routine maintenance. During routine maintenance, the tritium contamination may be such that with proper purging of the atmosphere, speciai precautions may not be needed. However, during major disassembly it will be necessary to provide tritium protection also. This will be true even if the interior is first hented under vacuum to drive off the absorbed tritium. We plan, then, that operators must not only be provided with lead shielding, but also possibly with a source of air for breathing. Appendix D discusses residual radioactivity, Appendix $\mathbf{G}$ discusses tritium problems in greater detail.

The reactor is at loast as cousplex as (for example) a research accelcrator. There must be routine maintenance and occasional disassembly. The prospect of working on such a machine from inside a shielded enclosure poses many problems. However difficult this situation may be, it is by no means impossible. As a rather elementary first approach, we have shown a crane with a shielded cab, and a shielded vehicle ("cherry picker") with appropriate manipulators. A serond shielded vehicle is required for rescuc purposes in case either the furt vehicle or the crane should fail. Each part of the machine must be very carefully designed with the thought in mind of removing it and replacing it remotely. Flanges, for instance, would probabiy not be of the simple bolted variety, but would have special quick-acting clamps. The possibility of using TV camera and remotely operated manipulators must be explored. For routine maintenance, local removable shiekding might offer some possibilities; however, for major disassemblies this does not appear feasible. A more detailed discussion of the requirements for remote assembly-disassembly procedures is given in Appendix $\mathbf{E}$.

The disassembly of the reactor is shown diaprammatically in Figs. 16a through $16 \mathrm{c}$.

\section{Reactor Building Complex}

The reactor buirding, described in detail in Appendix $K$, is highly specialized. It must contain the penetrating radiation from the operating reactor. The reactor containment vault shown in Fig. 17 must be 152-feet long, (14-feet wide, and 60-feet high to allow for convenient disassembly of the reactor. It is 10 feet thick, is gas-tight, and has a stainless-steel liner. The large access doors shown in Fig. 18 lead to assermbly and hot-disassembly areas at either end of the vault. Figure 18 also shows some of the facilities whicl are provided for preparation and irradiation of specimens and maintenance and repair of highly radiuactive equipment.

Space and utilities are provided for a lasge variety of reactor auxiliaries such as the DT fuel system, liquid helium refrigera'or, and injector power supplies, as well as appropriate office space and supporting shop facilities. Figures in Appendix $\mathrm{K}$ show building plans, sections, elevations, and an overall perspective view of the entire facility.

\section{Tritium System}

Tritium collected by cryopumps in the injectors and mirror loss regions is cycled through a purifier ( $\mathrm{Pd}-\mathrm{Ag}$ membranes) and isotopic separation plant (cryogenic distillation) and is reused in the injectors. An inventory of about $400 \mathrm{~g}$ is required in the system. Some tritium is generated by neutron capture in the boron used in the shield and will require periodic disposal. Care must be taken to outgas the equipment exposed to the plasma tritium prior to disassembly. The totel release of tritium during disassembly should be less than $0.1 \mathrm{~g}$.

During operation, the reactor vault would be filled with nitrogen to prevent the possibility of reactions between $T_{2}$ or $D_{2}$ and air. Low-level tritium confamination of the room nitrogen is removed by a tritiun recovery system (TRS). The major portion of the tritium system costs are associated with this main TRS. Accidental failure possibilities of the various tritium-containing systems have been considered. All aspects of tritium handling and safety can be managed using techniques and hardware which, in ruost cases, are known today. A detailed analysis of tritium reactor problems is contained in Appendix $G$. 


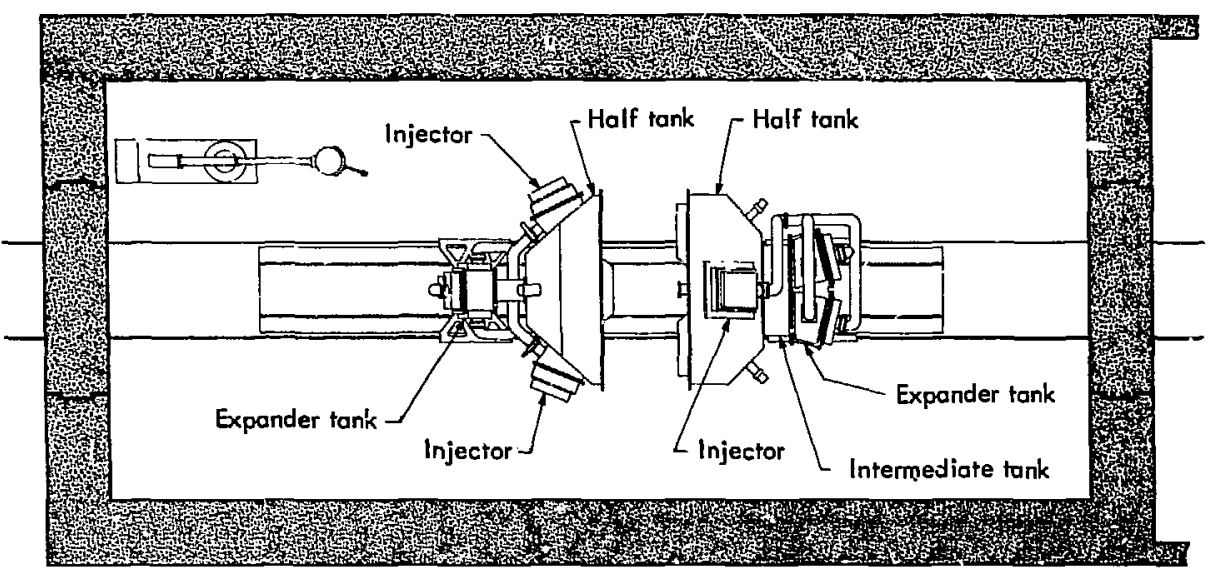

(a) Parting of reactar at midplane.

$m F_{1+1}^{5}$

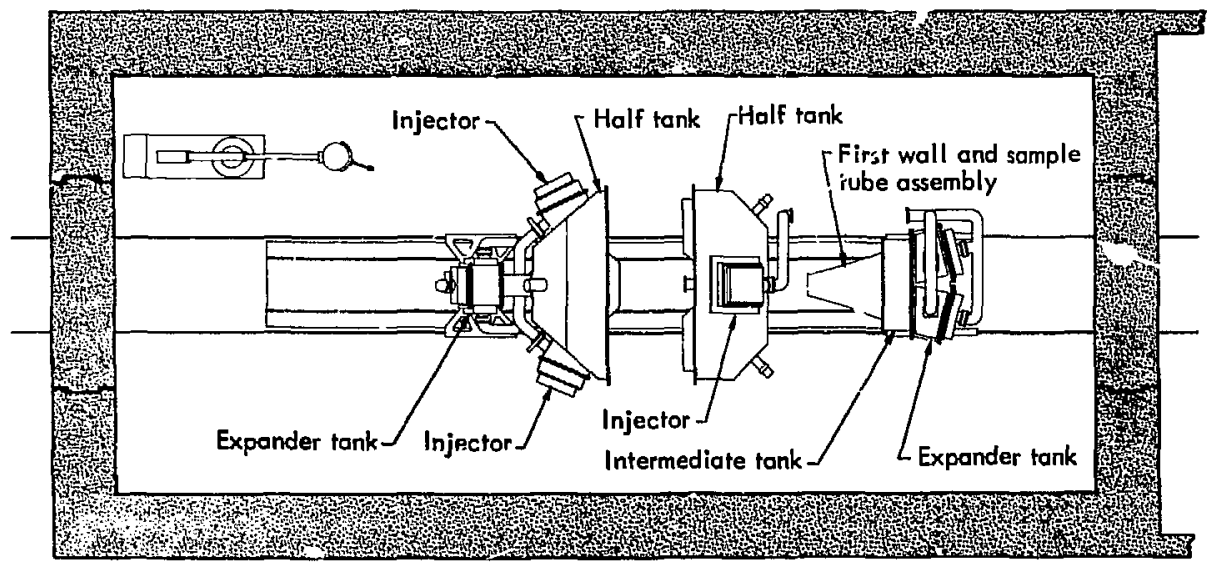

(b) Withdrawal af half of first wall, sample tube assembly, and assaciated infermediate $m[1+5]$ and expander tanks.

Fig. 16. Steps in disassembly of reactor. 


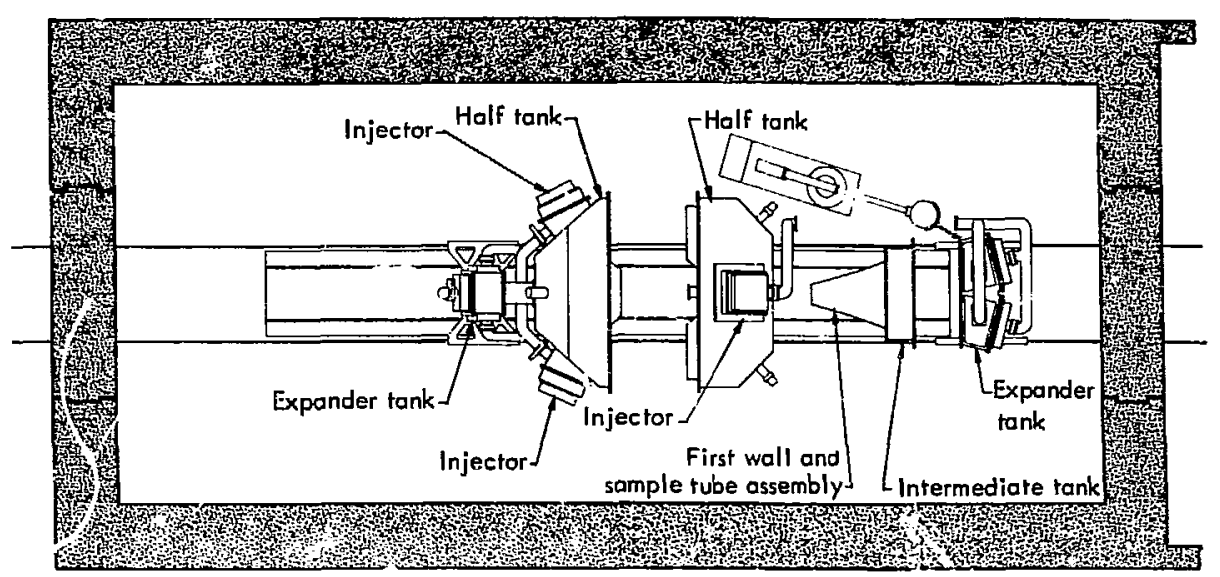

(c) Separation of expander runk.

$$
m[+1+1
$$

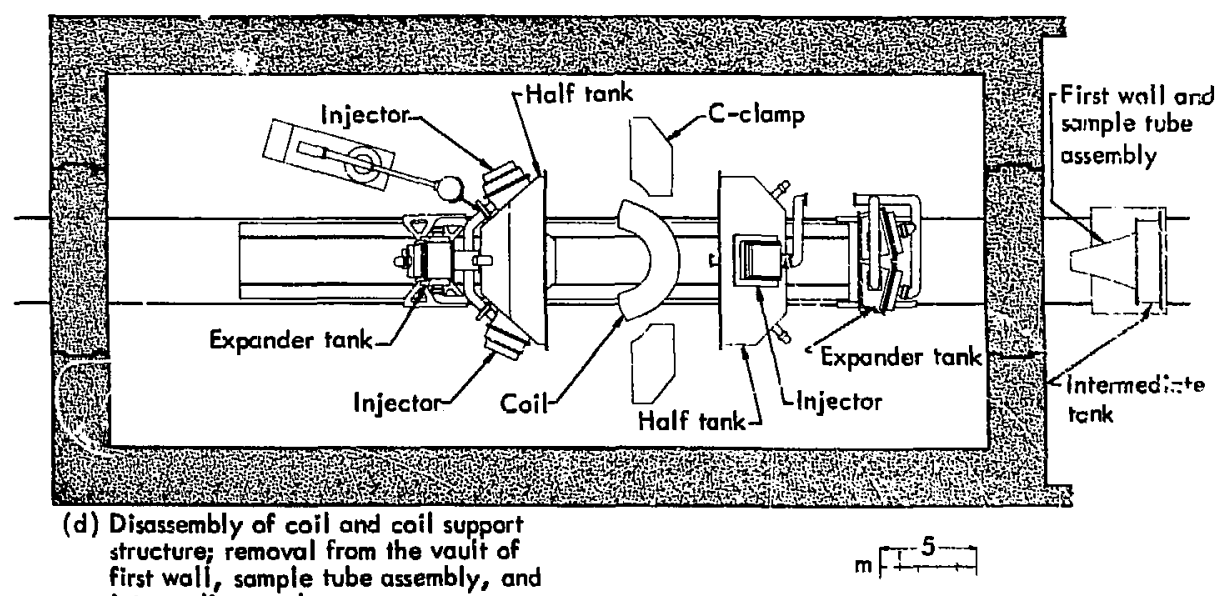

first wall, sample tube assembly, and intermediate tank.

Figure 16. Steps in disassembly of reactor iccontinued). 


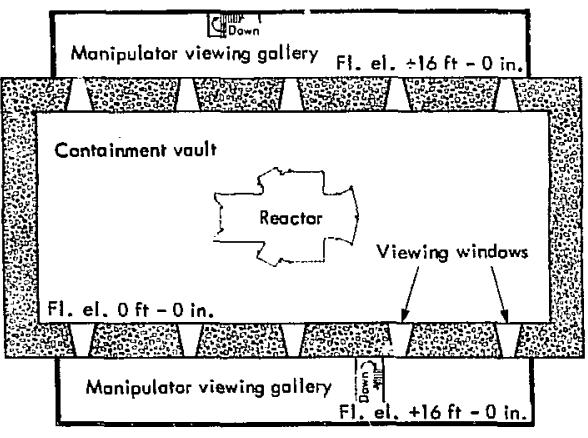

Fig. 17. Reactor in vault.

\section{COST ESTIMATE}

It must be emphasized that this cost estimate for a technologically advanced FERF facility is nade far in advance of both detailed engineering and essential laboratory technology development. Therefore, a significant uncertainty is implied and is reflected in part by a large $50 \%$ contingency applied to the reactor and its facilities.

Of the total cost of about 226 million dollars, the distribution among major components is shown in Table 3 (assuming equal distribution of contingency and engineering; design, inspection, and administration fees).

Table 3. Distribution of costs among major FERF components

\begin{tabular}{|c|c|c|}
\hline Item & Million $\$^{a}$ & $\%$ of total \\
\hline $\begin{array}{l}\text { Superconducting coil system } \\
\text { (including shiulding and } \\
\text { refrigeration) }\end{array}$ & 75.5 & 33.3 \\
\hline Neutral beain injectors & 44.3 & 19.6 \\
\hline Tritium system & 40.8 & 18.0 \\
\hline Other & 32.7 & 14.4 \\
\hline Utilities & 19.4 & 8.6 \\
\hline Building & 13.5 & 6.0 \\
\hline Totals: & 226.4 & 100.0 \\
\hline
\end{tabular}

${ }^{a}$ The costs are all in 1974 dollars, and an inflation factor tnust be applied for a future construction date.
A summary of the cost estimate is given in Table 4, divided into three sections; Reactor, Reactor Facilities, Building and Utilities. A more complete breakdown of the costs and a discussion of the cost estimating methods are given in Appendix $\mathbf{L}$.

\section{CONSTRUCTION SCHEDULE}

This reactor is in an early conceptual stage of engineering design, and any construction time schedule is approximate. Estimates can be made by comparing FERF with other construction projects of similar complexity and then making adjustments based on the state of technology. The resulting schedule allows 3 years of engineering, 5 years of construction, and 1 year of shakedown. If a serious proposal is made and engineering starts in FY 76, a schedule such as that shown in Fig. 19 could be followed, and the reactor could be operating in FY 85.* This schedule assumes that development work on major areas of technical innovation such as coils, materials, remote handling. etc., is successful and is vigorously pursued in parallel with engineering design.

Because of the large investment required to build FERF, certain design features must be proven before hund to ensure achieving the design guals (or at least to minimize the risk of failing). The primary goals are of an engineering rathar than a plasma physics nature, and to achieve these goals the plasma must perform to the designed value (for the low-field case).

- The magnet must deliver its designed field intensity because to fall short would drastically reduce the neutron flux (flux $\propto B^{4}$ ). Therefore, test magnets must provide high assurance that the magnet can in fact be built to specifications.

- The injectors must be developed. Fortunately, because of the modular nature of the injector system, a definitive test could be made on one module.

- The plasma performance, as measured principally by $\beta, n$, and $n \tau$, must provide a high assurance of achieving the desired goals.

*A major unknown is the time needed to satisfy licensing requirements. No similar device has ever been built. 

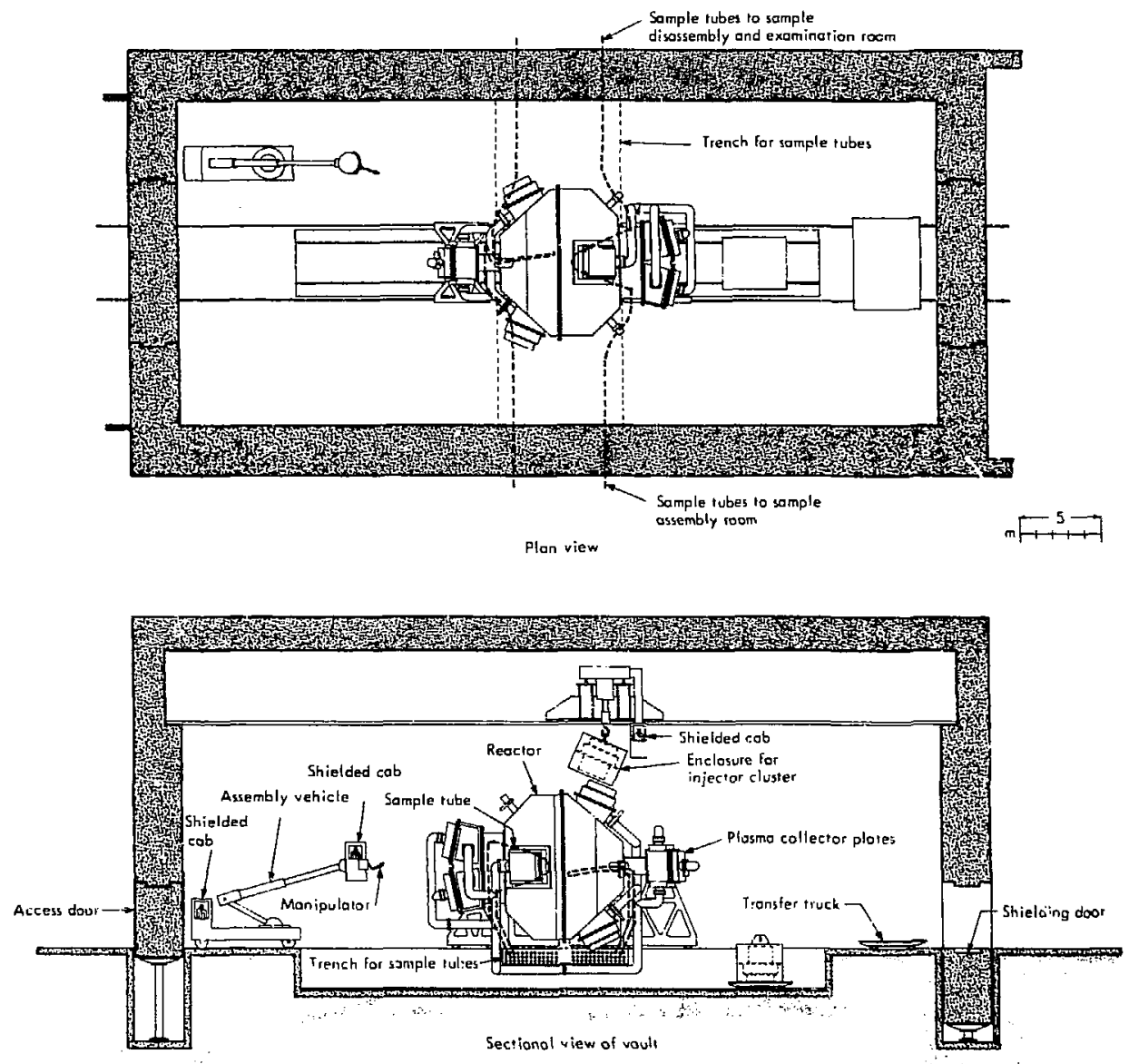

Fig. 18. Section and plan view of reactor in vault showing sample tubes passing through vault walls.

Physics experiments to determine the feasibility of FERF will be necessary under FERF-like conditions. However, by employing a hydrogen plasma and having a low duty cycle, there would be little or no radioactivity.

Achievement of an $\mathrm{nt}$ value of $\sim 1.6 \times 10^{12} \mathrm{~cm}^{-3} \mathrm{~s}$ with high energy ( $65 \mathrm{kcV})$, high density $\left(3 \times 10^{14} \mathrm{~cm}^{-3}\right)$, and large physical size (measured in $B_{\text {vac }} L \sim 16 \mathrm{~T} \cdot \mathrm{m}$ ) represents a considerable extrapolation from today's laboratory achievements $\left(n \sim 3 \times 10^{13}, H=I-10 \mathrm{keV}, \mathrm{n} \tau \sim 10^{10}\right)$. Current experiments (BBII and 2XIIB) can explore sone of these parameters, but not all at once because the fields are too low and the sizes too small. At least one generation of experiments beyond BBII and 2XIIB will be needed before the plasma physics will be well enough understood to minimize the risks of building FERF. Design of the next generation of mirror experiments is now in the early planning stages.

However, how close to the FERF design should these experiments be? If results from BBII and 2XIIB will be as good as those machines could give (given their size and field limitations), then one might be willing to rely on extrapolation to the FERF param- 
Table 4. Preliminary cost estimate summary.

Reactor:

Thousands of $\$^{\mathrm{a}}$

Superconducting coils (includes refrigeration, power supplies, radiation shield, thermal shicld, structure)

40,290

Injectors

First wall

Irradiation facilities

23,630

340

1,730

Plasma collector plates

Outer tanks

1,510

Support structure

1,100

Diagnostics

750

1,850

Controls and Instrumentation

800

Vacuum system

100

Cooling water distribution system

430

Models and tests

3,610

Total reactor construction:

Reactor facilities:

Tritium system

Ancillary equipment

Total reactor facilities construction:

Total reactor and reactor facilities

103,160

Engineering, design, inspection, administration, $25 \%$

25,790

Contingency, 50\%

64,480

Total reactor costs:

193,430

Building and utilities:

Building

10,390

Electrical substation

Water cooling system

7,100

Utilities and site improvements

Total building and utilities:

24,900

Architect-Engineer, inspection, $15 \%$

Contingency, 15\%

Total building and utilities:

${ }^{a} 1974$ dollars 

3. UWMAK-1, A Wisconsin Toroidal Fusion Reactor Design, Univ. of Wisc. Fusion Design Study Team, Rept. UWFDM-6̄8 (1973).

4. H. H. Barschall, Intense Sources of High Energy Neutrons, Lawrence Livermore Laboratory, Rept. UCRL-74031, (1972).
5. C. E. Taylor, Summary of a Conceptual Design of a Mirror Reactor for a Fusion Engineering Research Facility (FERF), Lawrence Livermore Laboratory, Rept. UCRL-75293 (1974).

6. R. W. Moir and W. L. Barr, Nuclear Fusion 13, 3S (1973). 


\section{Appendix A - Plasma Physics*}

The philosophy behind the FERF design is to see just how nearly classical the confinement of the plasma has to be in order to achieve the FERF goals while satisfying known plasma stability and equilibrium conditions. The discussion to follow will describe the plasma and the various constraints as we now understand them.

\section{PLASMA PARAMETERS}

We assume the plasma behaves in a way we will call "almost classical." In particular, the plasma is assumed to be stable to gross displacements (i.e., it is magnetohydrodynamically stable), and nicroinstabilities are assumed to be suppressed to such an extent and to be of such a character as to be describable as enhanced coulomb binary collisional losses.

The plasmn pressure perpendicular to the magnetic field, $\mathbf{P}_{1}$, is given as

$P_{\perp}=n_{D} \bar{w}_{D} \frac{2}{3} \gamma+n_{T} \bar{W}_{T} \frac{2}{3} \gamma+n_{e} \bar{w}_{e} \frac{2}{3}$,

where $n_{D}, n_{T}, n_{e}, \bar{W}_{D}, \bar{W}_{T}$, and $\bar{W}_{e}$ are the densities and menn energies of the deuterons, tritons, and electrons. $\mathrm{He}^{+}$ions are not confined. The factor $\gamma$ in Eq. (Al) accounts for the fact that more energy is perpendicular to the magnetic field in plasma confined by an ausotropic mirror than in plasma with an isotropic velocity distribution.

The plasma pressure which a given magnetic field configuration can hold is measured by $\beta$ where $\beta$ is defined as

$$
\beta_{1}=\frac{P_{\perp}}{\frac{B^{2} \text { vac }}{2 \mu_{0}}},
$$

where $B_{\text {yac }}$ is the vacuum magnetic field and $\mu$ is the permeability of free space.

"We acknowledge the help given by M. E. Rensink and A. H. Futch for their Fokker-Planck calculations, by C. C. Damm for larget plasma considerations, and by $J$. H. Foote for calculation of nonadiabatic particle energy limits.
In a mirror machine, $\beta$ can be quite high $(1 / 2<\beta<1)$ and is limited by the so-called mirtor, or firehose, instability. The most severely limiting instability is the mirror mode, which is stable if

$$
\frac{4 \pi}{\mu_{0}} \frac{\mathrm{dB}_{\mathrm{yac}}^{2}}{\mathrm{~dB}}=8 \pi \frac{\mathrm{B}}{\mu_{0}}+\frac{\mathrm{dP}_{1}}{\mathrm{~dB}}>0
$$

The ion density $\mathbf{n}$ is then given as

$$
\mathrm{n}=\frac{\beta_{\perp} \mathrm{B}_{\mathrm{vac}}^{2}}{2 \mu_{0}\left(\frac{1}{3} \gamma \overline{\mathrm{W}}_{\mathrm{D}}+\frac{1}{3} \gamma \overline{\mathrm{W}}_{\mathrm{T}}+\frac{2}{3} \overline{\mathrm{W}}_{\mathrm{e}}\right)} .
$$

where we assume $n_{D}=n_{T}=1 / 2 \mathrm{n}$. The thermonuclear power per unit volume is given as

$$
P / V=\frac{n^{2}}{4}(\tilde{o} \tilde{v})_{D T} E_{F},
$$

where $(\overline{\sigma v})_{\mathrm{DT}}$ is the reaction rate parameter averaged over the ion velocity distribution, and $E_{F}$ is the energy released in each fusion reaction. The total power, assuming $(\bar{\sigma} \bar{v})_{D T}$ is spatially constant, is

$P_{\text {fusion }}=\int_{\text {volume }} P / V d V=\frac{(\sigma v)_{D T} E_{F}}{4} \int n^{2} d V$.

The loss rate due to coulomb collisions equals the injection rate if charge-exchange losses are neglected and is given as

$$
I_{\text {loss }}=q \int \frac{n}{T} d V,
$$

where $\boldsymbol{r}$ is the mean lifetime of particles at density $\mathbf{n}$. If we multiply the numerator and denominator of Eq. ( $/ \mathbf{7})$ by $n$ and assume that the product $n r$ is independent of space, we get

$$
I_{\text {loss }}=\frac{q}{n T} \int_{\text {volume }} n^{2} d V
$$


The power gain $Q$ is defined as

$Q=\frac{P_{\text {fusion }}}{I_{\text {loss }} W_{0} / q}=\frac{(\bar{\sigma} \bar{q})_{D T}(n \tau) E_{F}}{4 W_{0}}$.

where $W_{0}$ is the injection energy. The number of neutrons produced per second $S$ is given as

$$
S=\frac{P_{\text {fusion }}}{E_{F}} .
$$

We will now find a set of parameters from Eqs. (Al) through (A10) corresponding to the low-field case. The high-field case can be scaled from these numbers.

We take $B_{\text {vac }}$ to be $3.75 \mathrm{~T}$ and $\beta$ equal to 0.65 . This value of $\beta$ was found from Eq. (A3) using the two-dimensional Fokker-Planck code and was just at the threshold of the mirror mode. To find the mean energies $\gamma$ and $n r$, we ran the one-dimensional FokkerPlanck multispecies code and the twortimensional, one-species Fokker-Planck code. The input for the one-dimensional code and the results are given in Table A-1. This code gives us the mean energies of the several species and $n t$ but does not give information on perpendicular pressure. The two-dimensional code gives angular information but not multispecies information. The input and key results from the latter are given in Table A-2.

Because of the importance of the density to alt aspects of the FERF, we show the calculation of density from $\mathrm{Eq}$. (A4)
Table A-I. Ont-dimensional multispecies FokktrPlanch input and results.
Input

$W_{D}$ injection $=65 \mathrm{keV}$

$\mathrm{W}_{\mathrm{T}}$ injection $=97.5 \mathrm{keV}$

$\mathbf{R}=\mathbf{2 . 3}$

Normal-mode angular distribution

$$
\begin{aligned}
& \text { Results } \\
& \mathrm{W}_{\mathrm{D}}=73.27 \mathrm{keV} \\
& \mathbb{W}_{\mathrm{T}}=93.03 \mathrm{keV} \\
& W_{\mathrm{e}}=7.17 \mathrm{keV} \\
& \mathrm{nT}=5.84 \times 10^{12} \mathrm{~cm}^{3} \cdot \mathrm{s} \\
& I_{D}=364 \mathrm{~A} \\
& I_{T}=136 \mathrm{~A} \\
& \mathrm{l}_{\mathrm{D}+\mathrm{T}}=500 \mathrm{~A} \\
& (\overline{\mathrm{OV}})_{\mathrm{DT}}=9.687 \times 10^{-16} \\
& (\overline{\mathrm{ov}})_{\mathrm{DD}}=1.8 \times 10^{-17} \\
& \mathrm{n}_{\mathrm{D}}=6.895 \times 10^{12} \mathrm{~cm}^{-3} \\
& n_{T}=6.2329 \times 10^{12} \mathrm{~cm}^{-3} \\
& \mathrm{n}_{\mathrm{e}}=13.2402 \times 10^{12} \mathrm{~cm}^{-3} \\
& J_{D}=2.183 \times 10^{13} \mathrm{~cm}^{-3} \mathrm{~s}^{-1} \\
& \mathrm{~J}_{\mathrm{T}}=0.817 \times 10^{13} \mathrm{~cm}^{-3} \mathrm{~s}^{-1} \\
& J_{e}=3.0 \times 10^{13} \mathrm{~cm}^{-3} \mathrm{~s}^{-1} \\
& \mathrm{Q}_{\mathrm{TT}}=0.417 \\
& \phi_{\mathrm{P}}=24.3 \mathrm{kV}
\end{aligned}
$$

$$
\begin{aligned}
& \mathbf{n}=\frac{0.65(3.75)^{2}}{2 \times 4 \pi \times 10^{-7}\left(\frac{1}{3} \times 1.3 \times 73.27+\frac{1}{3} \times 1.3 \times 93.03+\frac{2}{3} \times 7.17\right) \times 10^{3} \times 1.6021 \times 10^{-19}} \\
& \mathbf{n}=2.954 \times 10^{14} \mathrm{~cm}^{-3}
\end{aligned}
$$

With the above density, the central power density is $59.3 \mathrm{~W} \cdot \mathrm{cm}^{-3}$ of neutrons and alpha particles. The fraction of $\beta$ contributed by each species is

$$
\frac{\beta_{\mathrm{D}}}{\beta}=0.413 ; \frac{\beta_{\mathrm{T}}}{\beta}=0.525 ; \frac{\beta_{\mathrm{e}}}{\beta}=0.062
$$

The plasma is assumed to be cylindrically symmetric at the midplane $(z=0)$ and to have a radial density profile as compared with the buillup code, discussed later. Emperical curve fits have shown this radial profile to be best approximated by the following cubic:

$$
\mathrm{n}(\mathrm{r})=\mathrm{n}_{00}\left(1-\left(\frac{\mathrm{r}}{\mathrm{R}}\right)^{3}\right) .
$$

The axial profile is that calculated by the twodimensional Fokker-Planck code which computes $\mathbf{n}$ as 
Table A-2. Two-dimensional Fokker-Planck results.

\begin{tabular}{llll}
\hline & & \multicolumn{2}{c}{ Radius $(\mathrm{cm})$} \\
\cline { 2 - 4 } Parameter & 0.0 & 12.5 & 17.5 \\
\hline$W_{\mathrm{o}}$ injection $\mathrm{D}^{+}(\mathrm{keV})$ & 65 & 65 & 65 \\
$\mathrm{~W}_{\mathrm{D}}(\mathrm{keV})$ & 63.82 & 64.97 & \\
$\mathrm{~W}_{\mathrm{e}}(\mathrm{keV})$ & 6.26 & 6.28 & \\
$\phi \mathrm{p}(\mathrm{kV})$ & 21.2 & 21.1 & \\
$\mathrm{nr}\left(\mathrm{cm}^{3} \cdot \mathrm{s}\right)$ & $4.971 \times 10^{12}$ & $5.13 \times 10^{12}$ & $4.807 \times 10^{12}$ \\
$\mathrm{R}$ & 3.38 & 3.05 & 2.642 \\
Injection angles (degrees) & $55,63,70,78$ & $60.68,76.83$ & $63,71,78,86$ \\
$\gamma$ & 1.302 & 1.33 & \\
$\beta_{\perp_{\mathrm{crit}}}$ & 0.679 & 0.5745 & \\
$\beta_{\perp}$ & 0.65 & 0.569 & 0.427 \\
$\mathrm{n}\left(\mathrm{cm}^{-3}\right)$ & $2.954 \times 10^{14}$ & $2.586 \times 10^{14}$ & $1.262 \times 10^{14}$ \\
\hline
\end{tabular}

a function of $B$. When we make the long slender approximation (only approximately true for our case) which says that axially the density varies slowly, we can show that

$$
B=B_{\text {vac }} \sqrt{1-\beta}=B_{\text {vac }} \sqrt{i-\beta_{0} \frac{P_{1}(\beta)}{P_{L_{0}}}} .
$$

We calculate the vacuum magnetic field with the computer code MAFCO which gives $B_{\text {vac }}$ as a function of space. The spatial variables we take are the midplane radius $r$ and angle $\theta$ and the distance along a field line s. Figure A-l shows the assumed radial and calculated axial density profiles. Figure A-2 shows the axial vucuum magnetic ficld and the field which is depressed by the plasma diamagnetism calculated from the two-dimensional code results and Eq. Al4. Figure A-3 shows the same things in the radial direction at the midplane. Figure $A-4$ shows vacuum magnetic field lines and density profiles.

To compute the power produced and the injection current required, we evaluated Eq. (A8) numerically by breaking the volume integral into two integrals: an area integral at the midplane which cut tubes of plasma bounded by magnetic fux lines, and in integral along the flux tube measured by the variable s. The volume integral then became

$$
\int n^{2} d V=\int_{0}^{R} \int_{-\frac{L}{2}}^{\frac{L}{2}} n^{2} 2 \pi r \frac{B_{0}}{B} d r d s,
$$

where $\mathrm{B}_{\mathbf{0}}$ is the magnetic field at the midplane, and $B$ is the value at position $S$ along the flux tube. Thus, the ratio $\mathrm{B}_{0} / \mathrm{B}$ takes into account the area contraction due to $B$ increasing towards the magnetic mirrors. The integral in Eq. (A11) has been put into a standard form as follows:

$G^{i}=\int_{0}^{R} 2 \int_{0}^{L / 2} \frac{2 \pi t}{\pi R^{2} L}\left(\frac{n}{n_{00}}\right)^{i} \frac{B_{0}}{B} d r d s$

where $i$ is a superscript of $G$ rather than a power.

The numerical integration divided $L$, the length betwcen mirrors $(420 \mathrm{~cm})$, into $10-\mathrm{cm}$ intervals, with

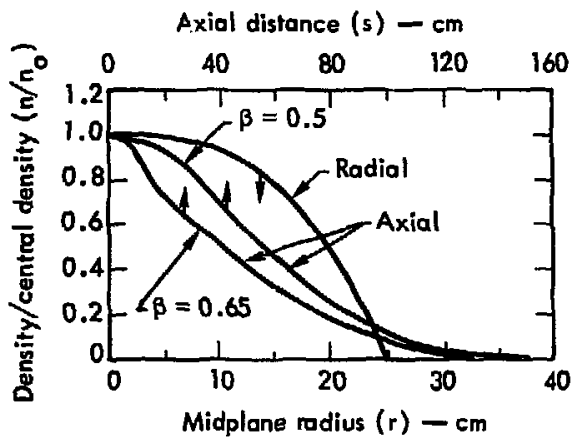

Fig. A-1. Radial and axial density protiles for $\beta=0.5$ and the design value of $\beta=0.65$. 


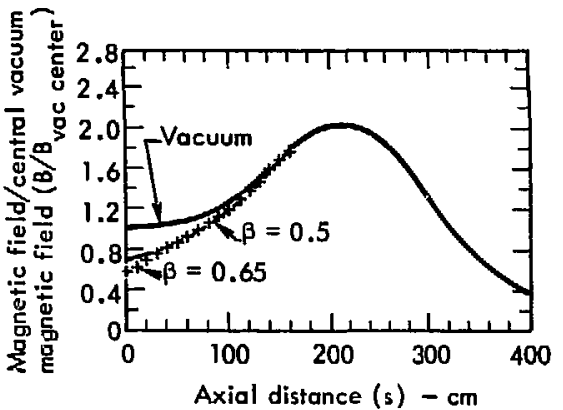

Fig. A-2. Axial magnetic field profile for $\beta=0$ (vacuum field), 0.5 , and 0.65 .

radial points at $r=0,8,12.5,17.5$, and $25 \mathrm{~cm}$. The results were

$$
G^{0}=0.6494, G^{l}=0.13589, G^{2}=0.06887
$$

The plasma volume, $\mathrm{V}$, is

$$
V=\Pi R^{2} L G^{0}=535.51 .
$$

The total number of confined ions, $N$, is

$N=\Pi R^{2} L n_{00} G^{1}=1.1206 \times 10^{5} n_{00}=3.310 \times 10^{19}$.

For $\mathrm{n}_{00}=2.954 \times 10^{14} \mathrm{~cm}^{-3}$, which is the central density for the low-field case, the value of the integral

$$
\begin{aligned}
\int n^{2} d V & =\pi R^{2} L n_{00}^{2} G^{2}=5.680 \times 10^{4} n_{00}^{2} \\
& =4.956 \times 10^{33} \mathrm{~cm}^{-3}
\end{aligned}
$$

The power of $14-\mathrm{MeV}$ neutrons plus $3.52-\mathrm{MeV}$ He is, from Eq. (A6)

$$
\begin{aligned}
P= & \frac{4.956 \times 10^{33} 9.687 \times 10^{-16}}{4} \\
& \times \frac{17.58 \times 10^{6} \times 1.6021 \times 10^{19}}{4} \\
P= & 3.38 \times 10^{6} \mathrm{~W}
\end{aligned}
$$

The classical loss rates will now be calculated for $\mathrm{D}^{+}$and $\mathrm{T}^{+}$separately instead of using Eq. (A8):

$$
\begin{aligned}
I_{l o s s} D^{+} & =q \int \frac{n_{D}}{\tau_{D}} d v \\
& =q \int J_{D} d v \\
I_{l o s s} T^{+} & =q \int J_{T} d v .
\end{aligned}
$$

Now, from Table A-1 we have J, the source current per unit volume at the densities in the table. Since $J \propto n^{2}$, we have

$$
\mathrm{J}_{0 \mathrm{D}}=2.183 \times 10^{13}\left(\frac{2.954 \times 10^{14}}{13.2403 \times 10^{12}}\right)^{2}
$$

$$
=1.0867 \times 10^{16} \mathrm{~cm}^{-3}
$$

$\mathrm{J}_{0 \mathrm{D}}=0.817 \times 10^{13}\left(\frac{2.954 \times 10^{14}}{13.2403 \times 10^{12}}\right)^{2}$

$$
\begin{array}{r}
=0.407 \times 10^{16} \mathrm{~cm}^{-3} \\
I_{D}=q J_{0 D} \int \frac{n^{2} d v}{n_{0}{ }^{2}}=J_{0 D} \pi R^{2} L \int\left(\frac{n}{n_{00}}\right)^{2} \frac{d v}{\pi R^{2} L}
\end{array}
$$

$$
=1.6021 \times 10^{19} \times 1.0867 \times 10^{16} \pi 25^{2} 420
$$

$$
\times 0.06887
$$

$$
=98.88 \mathrm{~A}
$$

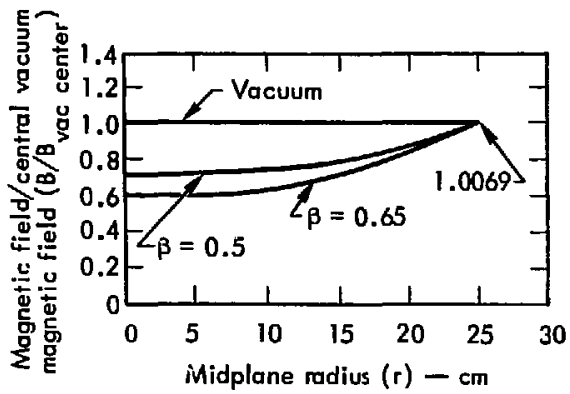

Fig.A-3. Radial magnetic field profile for $\beta=0,0.5$, and 0.65 . 


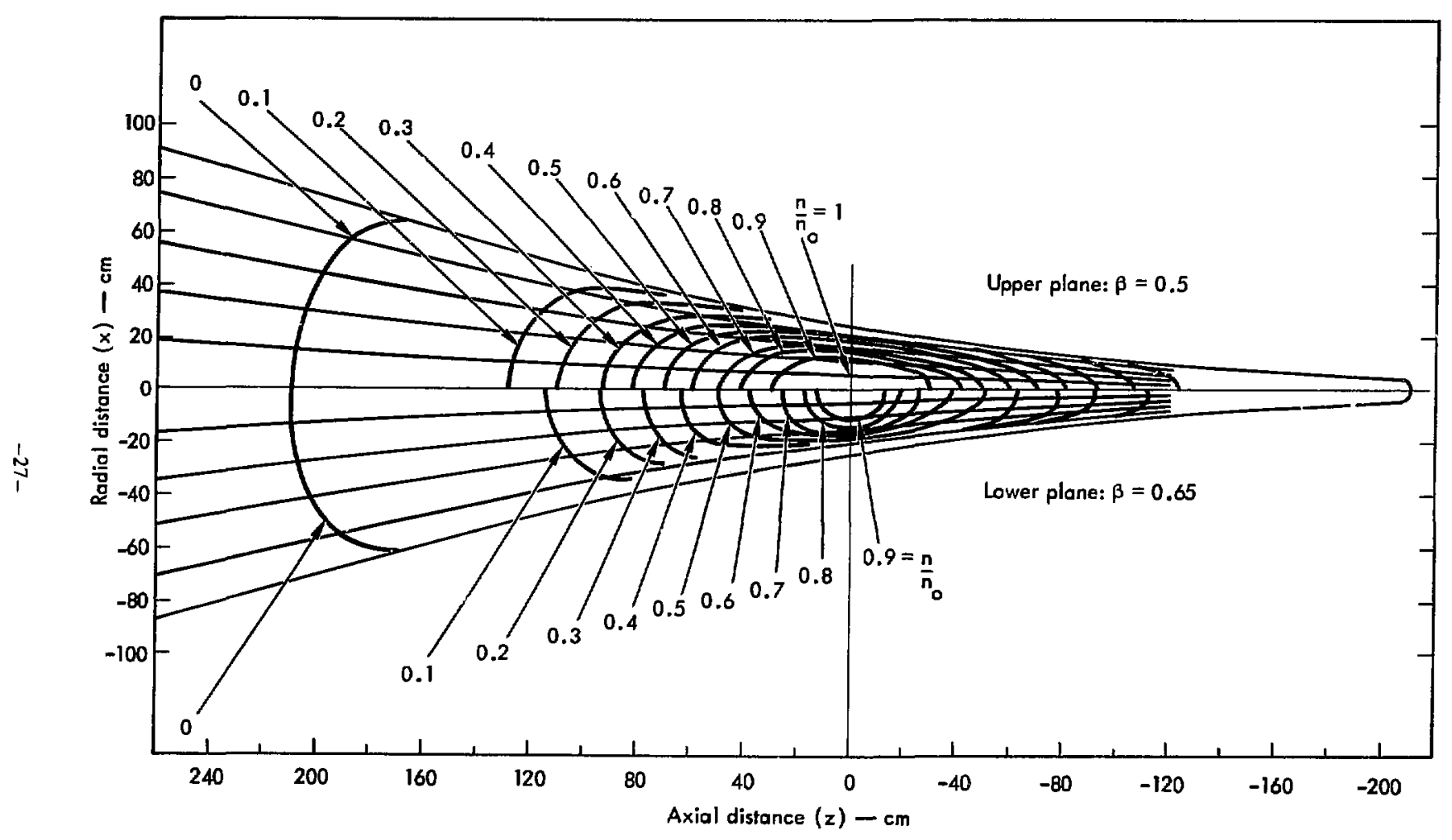

Fig. A-4. Density contours (for $\beta=0.5$ and $\beta=0.65$ ) and vacuum magnetic ficld lines. 


$$
I_{T}=37.03 \mathrm{~A}
$$

$$
\mathrm{I}=\mathrm{I}_{\mathrm{D}}+\mathrm{I}_{\mathrm{T}}=135.9 \mathrm{~A}
$$

$P_{\text {classical }}=37.03 \times 97.5 \times 10^{3}+98.88 \times 65$

$$
\times 10^{3}=10.04 \mathrm{MW}
$$

$\mathrm{Q}(17.58 \mathrm{MeV})=\frac{3.38}{10.04}=0.337$

The actual injection power for $500 \mathrm{~A}$ iniected (the capability selected for the present design) is

$P=136 \times 97.5 \times 10^{3}+364 \times 65 \times 10^{3}=36.92 \mathrm{MW}$

The trapping efficiency is calculated to be approximately $84 \%$, giving a trapped injected beam capability of $31.38 \mathrm{MW}$. The design $Q$ is then $3.38 / 31.38=0.11$ The classical average $\mathbf{n} \boldsymbol{\tau}$ for deuterons and tritons is given as

$$
\begin{aligned}
n \tau_{\text {classical }}= & \frac{\mathrm{n}^{2}}{\mathrm{~J}_{\mathrm{D}}+\mathrm{J}_{\mathrm{T}}}=\left(\frac{13.24 \times 10^{12}}{3 \times 10^{13}}\right)^{2}=5.84 \\
& \times 10^{12} \mathrm{~cm}^{-3} \mathrm{~s}
\end{aligned}
$$

Thus, the actual injection power is 3.1 times grenter than that required to sustain classical losses; and the 500-A capability could sustain an no of $1.9 \times 10^{12}$ $\mathrm{cm}^{-3} \mathrm{~s}$, an amount 3.1 times less than the classical nr.

This result can be compared with the classical injection current, $I_{\text {loss }}$, calculated irom Eq. (A8) for a onespecies plasma ( $\mathrm{D}^{+}$only):

$$
\begin{aligned}
l_{\text {loss }}= & \frac{q}{n \tau} \int n^{2} d V=\frac{1.6021 \times 10^{-19}}{5 \times 10^{12}} 4.956 \\
& \times 10^{33}=158.8 \mathrm{~A} .
\end{aligned}
$$

This is 3.15 times less than the 500-A design capability. The classical injection at $65 \mathrm{keV}$ would be $10.3 \mathrm{MW}$; therefore, the classical $\mathrm{r}(17.58 \mathrm{MeV})$ is 0.328 . We see that treating the plasma with one species overestimates the losses somewliat.
The source of $14-\mathrm{MeV}$ neutrons is

$$
\begin{aligned}
& S=\frac{4.956 \times 10^{33} \times 9.687 \times 10^{-16}}{4} \\
& S=1.2 \times 10^{18} \mathrm{~ns}^{-1} .
\end{aligned}
$$

If this were a point source, the flux on a sphere at 25 -cm radius would be

$$
\Gamma=\frac{S}{4 \pi R^{2}}=1.53 \times 10^{14}{\mathrm{n} \cdot \mathrm{cm}^{-2} \cdot \mathrm{s}-1}^{-1}
$$

If the source were uniformly spread throughout a 25 cm-radius sphere, the current would be the same as above but the flux would be 1.5 times larger, or

$$
\Gamma=2.29 \times 10^{14} \mathrm{~cm}^{-2} \mathrm{~s}^{-1} .
$$

The spatial dependence of the flux of 14-MeV neutrons is calculated by first deriving a line source

$$
F(s)=\frac{(\bar{\sigma} \bar{v})}{4} \int T \frac{n^{2}}{2 \pi r d r} \frac{B}{B_{0}} .
$$

Then, the calculated flux is

$$
\Gamma(r, s)=\int_{-L / 2}^{L / 2} \frac{F(s)}{4 \pi r^{\prime 2}}, d s
$$

where $r$ ' is the distance from the element ds to the point at $r, s$. The line source $F(s)$ and $\Gamma$ at several positions are given in Figs. A-5 and A-6. At $Z=0$ and $\mathrm{r}=35 \mathrm{~cm}$, samples could be placed in the maximum flux $\left(0.531 \times 10^{14}\right.$ neutron $\left.\mathrm{cm}^{-2} \cdot \mathrm{s}^{-1}\right)$. At the edge of the plasma where $r=25 \mathrm{~cm}$, the flux is $0.89 \times 10^{14}$.

Another calculation which assumes the axial profile to be the same on each line but otherwise calculates flux properly shows that the line source caiculation gives a lower neutron flux than does a volume source close to the plasma. This correction is $8 \%$ at $35 \mathrm{~cm}$ and $20 \%$ at $25 \mathrm{~cm}$. The flux at $35 \mathrm{~cm}$ is then $0.57 \times 10^{14} \mathrm{~cm}^{-2} \mathrm{~s}^{-1}$ and $1.07 \times 10^{14} \mathrm{~cm}^{-1} \mathrm{~s}^{-1}$ at a $25-\mathrm{cm}$ radius. 


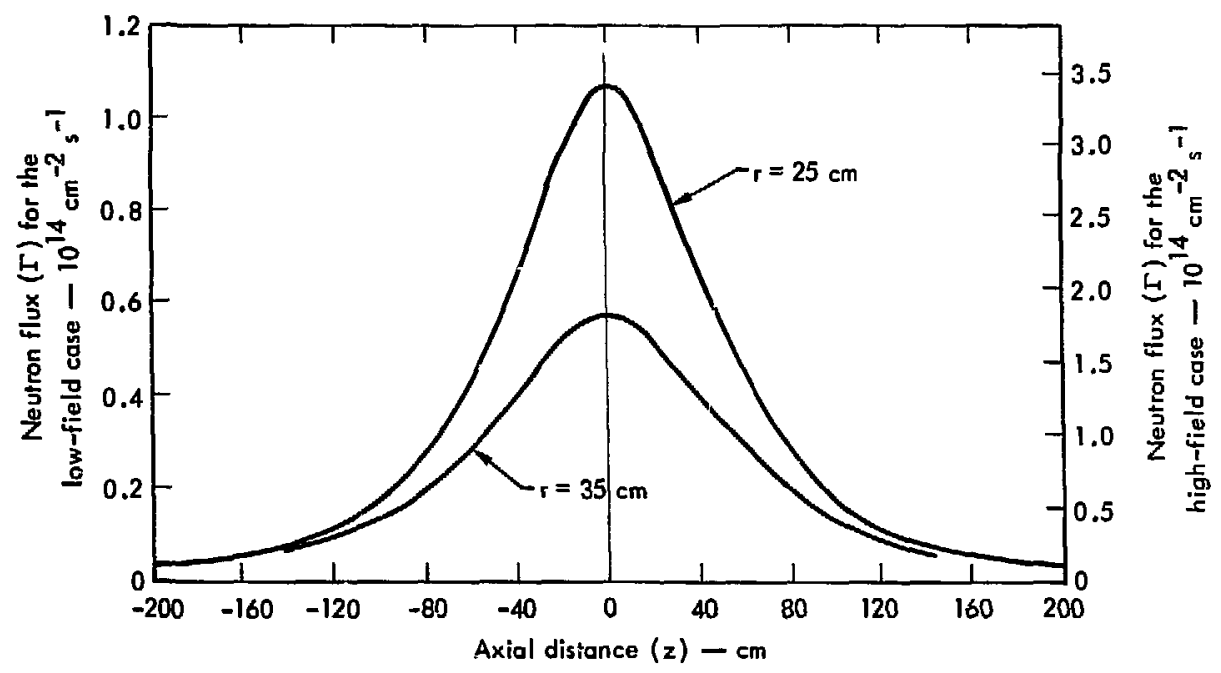

Fig. A-5. 14-MeV neutron flux versus axial position at 25 and $35-\mathrm{cm}$ radius for $\beta=0.65$.

\section{ENERGY SCALING}

The 14-MeV neutron flux at the surface of thi: FERF plasma varies as

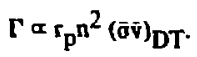

For constant magnetic field strength and $\beta$,

$$
n \propto \frac{1}{w_{\text {inj }}} .
$$

Therefore,

$$
\Gamma \propto \frac{r_{p}^{(\overline{o v})} D T}{W_{i n j}^{2}} .
$$

Because the size of the plasma has a strong influence on magnet size and beam power (both are major cost items), first consider the energy scaling of FERF with a constant plasma radius. FERF should have the minimum plasma size which will provide sufficient space for $\mathrm{p}:$ :rforming irradiation tests. For this study. the minimum size is judged to be a radius at the plasma midplane of about $25 \mathrm{~cm}$. The neutron flux, $\Gamma$, is plotted as a function of injection energy $W_{i n j}$, in Fig. A-7 for the design point case of $r_{p}=25 \mathrm{~cm}$. Note that at the design point of $W_{i n j}=65 \mathrm{keV}$, the neutron flux is $1.08 \times 10^{14} \mathrm{~cm}^{-2} \mathrm{~s}^{-1}$. The curve shows that the neutron flux increases rapidly with decreasing injection energy and is maximum at $W_{\text {inj }}=20 \mathrm{keV}$. Unfortunately, a $20-\mathrm{keV}$ injection energy cannot be used with $r_{p}=25 \mathrm{~cm}$ because the required injection power is too large and the beam will not penetrate to the interior of the plasma.

The injeciion current which must be trapped ty the plasma varies as

$$
I_{\text {inj }} \propto \frac{n r_{p}^{3}}{\tau}=\frac{n^{2} r_{p}^{3}}{n r},
$$

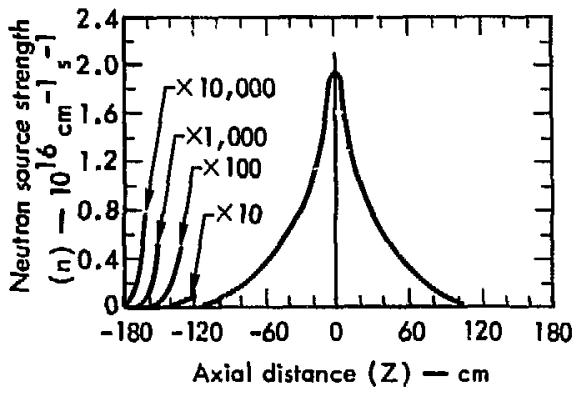

Fig. A-6. Calculated equivalent line souree of 14-MeV neutrons. 




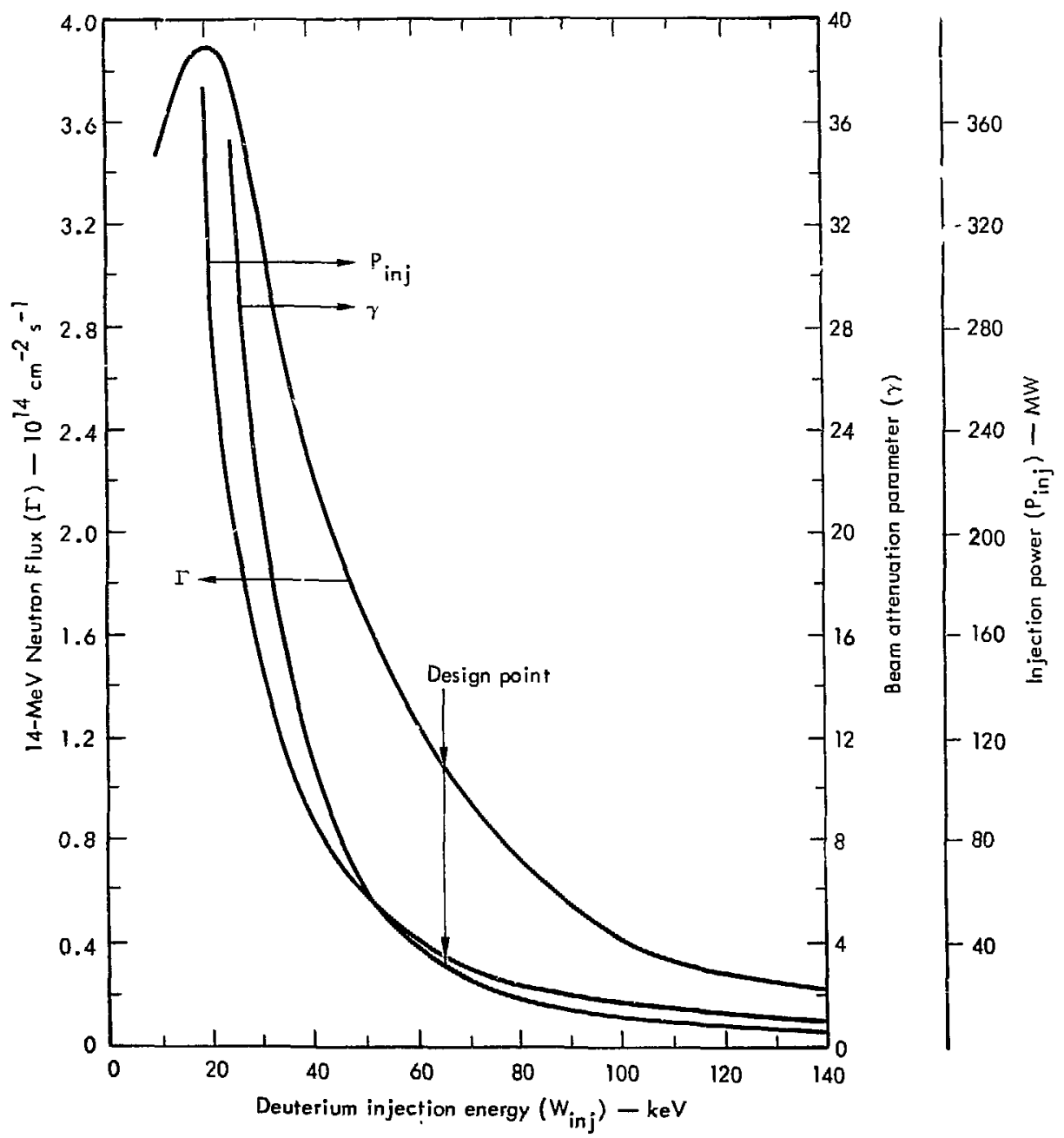

Fig. A-7. Neutron flux and neutral atom power flux to the wall versus injection energy at constant plasma $t a d i u s, r_{p}=25 \mathrm{~cm}$. 



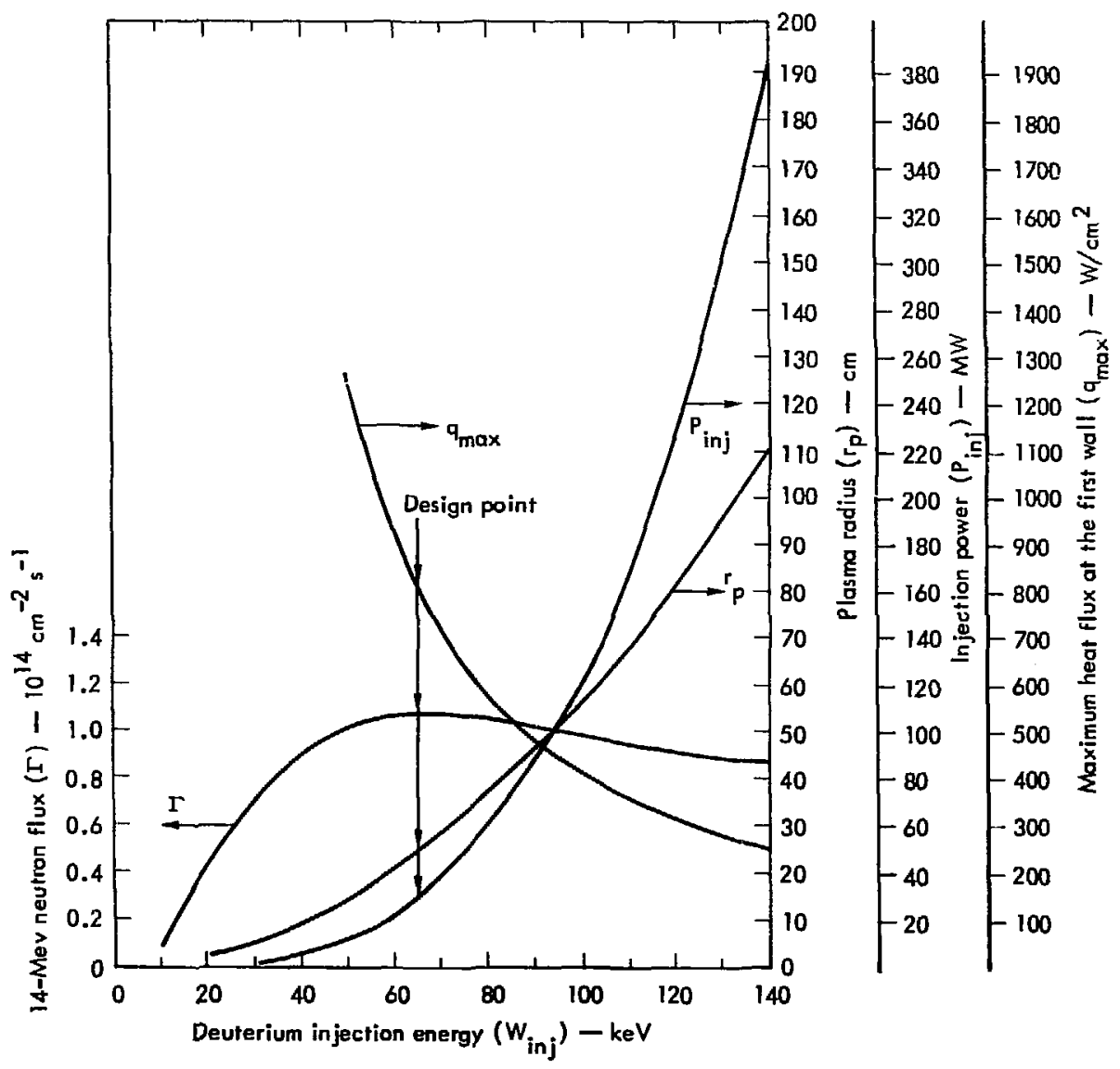

Fig. A-8. Neutron flux, neutral atom power flux to the first wall, classical loss current. plasma radius vs injection energy at constant beam attenuation parameter, $\gamma=3.5$. 


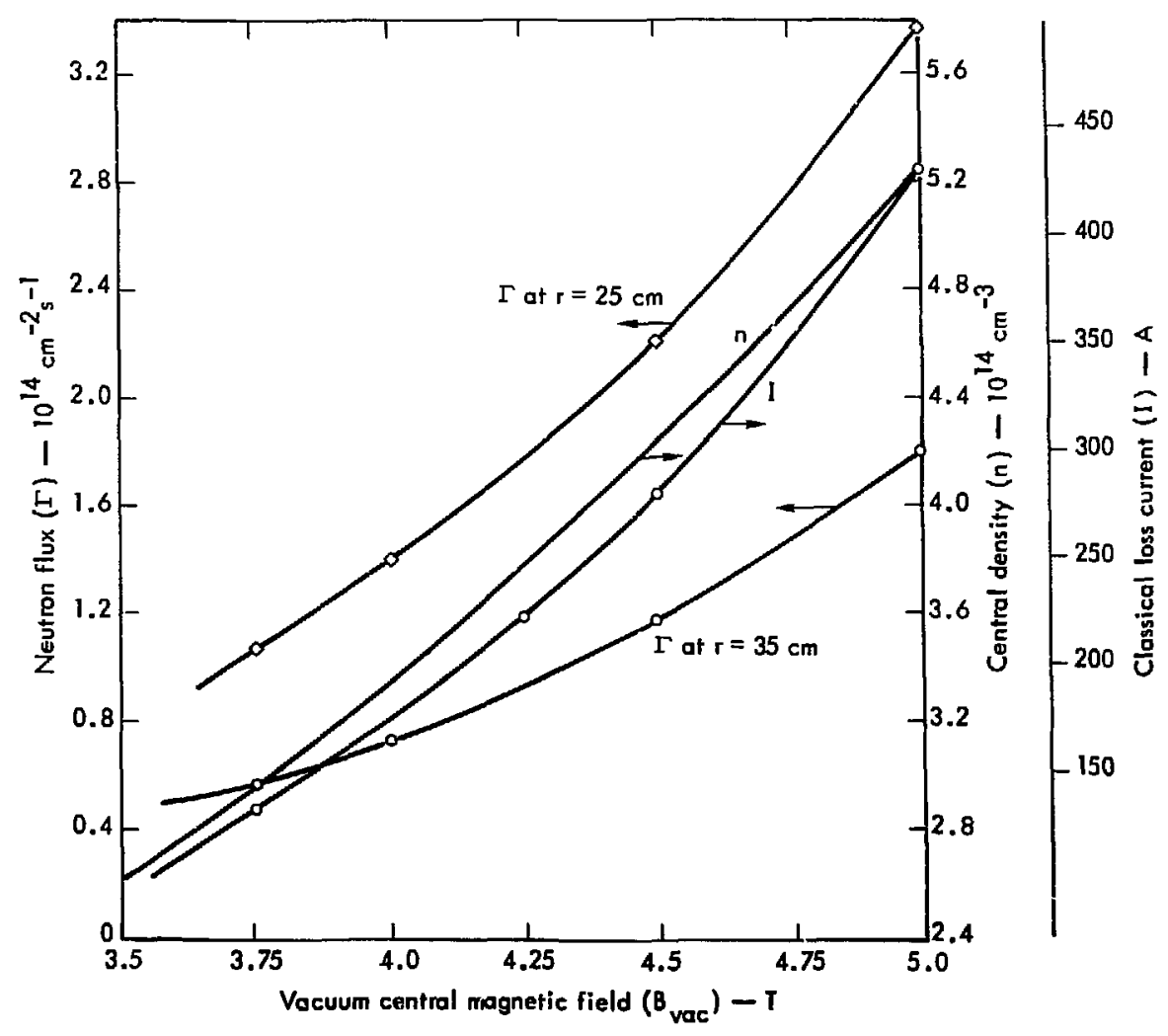

Fig. A-9. Neutron flux, central density, and classical loss current veraus central vacuun magnetic field. 
and lowering the mirror ratio or by raising the field uniformly everywhere up to the high-field value of $12.0 \mathrm{~T}$ at the conductor.

\section{PLASMA STABILITY}

Our basic assumption about the plasma is that it has a well defined spatial equilibrium and that the only appreciable losses result from coulomb collisions which cause scattering into the velocity-space loss cone and instabilities which are limited to not more than a factor of two more losses than the collisional losses. We will mention three classes of instabilities: mirror or firehose mode, interchange mode, and microinstabilities

The mirror mode is an instability, or possibly a loss of equitibrium, which can occur when the plusma presst re is strong enough to make a local field depression which locally confines more particles and leads to a further field depression in an unstable way. This mode can be avoided by not exceeding a critical plasma pressure or a critical $\beta$. For our case, the critical $\beta$ was 0.68. Since this mode is a local instability, the distribution function can be tailored by orientating injectors to optimize $\beta$ by smoothing the pressure gradients at the critical positions. So far, the angular orientation of the injectors las not buen optimized to maximize $\beta$. Future work should explore this optimization.

The interchange mode. a gross movemen: of the plusma ucross the magnetic fictd, is suppressed by providing a radial magnelic well and is the purpose for the unusually shaped Yin-Yang coil. Many experiments have shown the effectivesess vi radially increasing mugnetic fictus (good curvature) even at $\beta$ valuss of stverul tenths. The plasma diumagnetism causes the magnetic ficld lines near the midplane to bulge out. thus tending to add bad field-line curyature. This effect is somewhat compensated for by the increased magnetic gradient in the bulk of the plasma. The uncertainty is quantitatively how high can $\beta$ be. Af present. a computational physics effort is underway to determine the limiting $\beta$. If the limiting $f$ due to the interchange mode is less than the assumed 0.65 (as we are fairly certain will be the case), then either the coil must be redesignet to give more radial well or the ceneral feld strength must be raised to bwer? without reducing the neutran output.

Finally, the high-frequency ( $\omega>\omega_{\mathrm{ci}}$ ) instabilitics known as microirstabilities must be sufficiestly suppressed. Our designs allow for the less rate to be enhanced over the classical rate by up to a factor of three. One recent theory ${ }^{A l}$ suggests that at high densities the anomalous losses are indeed within o factor of two to three of the classical losses. Recent 2XIl experimental results partially support this theory in that at low ion energies $(\sim 1-3 \mathrm{keV})$ the losses were near classical. However, as the energy was raised, the losses remained about constant whereas the clussical loss rate decreases as $\mathbf{W}^{-3 / 2}$.

The cent ral density of $2.95 \times 10^{14} \mathrm{~cm}^{-3}$ and the magnetic field with plasma of $3.75 \sqrt{1-0.65}=2.22 \mathrm{~T}$ gives an electron dielectric constant $\left(\omega_{\mathrm{pe}} / \omega_{\mathrm{ce}}\right)^{2}=6.2$ and an ion dielectric constant $\left(\omega_{\mathrm{pi}} / \omega_{\mathrm{ci}}\right)^{2}=2.3 \times 10^{4}$ for $\mathrm{D}^{+}$ions. Theory indicates that $\left(\omega_{\mathrm{pe}} / \omega_{\mathrm{ce}}\right)^{2} \geq 1$ is perhaps a necessary condition for suppression of instability losses. If the central density should drop by a factor of two, then $\left(\omega_{\mathrm{pe}} / \omega_{\mathrm{ce}}\right)^{2}=1.6$; that is, $\left(\omega_{\mathrm{pe}} / \omega_{\mathrm{ce}}\right)^{2}=n \mathrm{~m}_{\mathrm{e}} / \epsilon_{0} \mathrm{~B}_{\mathrm{vac}}^{2}(1-\beta)$. The latter shows the importance of high- $\beta$ operation for suppression of microinstabilities.

The axial and radial sizes of a mirror plasma play a role in many theoretical criteria and are therefore given here for general information. The gyroradius, $a_{j}$, at the center is $2.45,3.39$, and $12.0 \mathrm{~cm}$, assuming the 73-keV D+, 93-keV $\mathrm{T}^{+}$and $3.52-\mathrm{MeV} \mathrm{He}^{++}$, are perpendicular to the field. The ratio of plasma radius, $t_{p}$, and iength between mirrors, $L$, to the central $\mathrm{D}^{+}$gyroradius (taking the above energy perpendicular to the field) is $r_{p} / n_{i}=10.2$ and $L / a_{i}=171$.

Perhaps the biggest unknown and threat to FERF is the role of instabilities. This is one of the main arguments for a large scale experiment to demonstrate the feasibility of the FERF. For the rest of this discussion of plasma physics, we will assume instabilities are adequately suppressed.

\section{ADIABATICITY}

The adiabatic confinement principle states that if the mugnutic field varies slowly in one gyroperiod, then the magnetic moment is conserved and long-time confinement can result. For the FERF magnetic field. one quickly finds that the spatial magnetic field variation is small without the plasma but that the plesma depresses the field and makes rather strong field gradients particularly in the radial direction. 
Foote ${ }^{A 2}$ has discussed the adiabatic limit and gives the following formula for the maximum energy which can be contained.

$$
\begin{aligned}
& W_{\max }(\mathrm{keV})=\left(8.5 \times 10^{-4}\right)\left(\frac{z^{2}}{A}\right) \frac{\left(B_{\left.M^{L}\right)^{2}}\right.}{R(R-1)^{3}} \\
& \frac{1}{L^{2}}=\frac{1}{L_{z}^{2}}+\frac{1}{L_{R}^{2}(R-1)} .
\end{aligned}
$$

The magnetic field is fitted by the following expression:

$$
B=\frac{B_{M}}{R}\left[1+\left(\frac{z^{2}}{L_{z}^{2}}+\frac{r^{2}}{L_{r}^{2}}\right)(R-1)\right] .
$$

$\mathrm{B}_{\mathrm{M}}$ is the mirsor field and is $7.58 \mathrm{~T}$ for FERF. Fits made to both the magnetic field and the mirror field in the radial and axial directions are shown in Figs. $A-10$ and $A-11$ for $\beta=0.5$ and 0.65 . The mirror ratio was 2.9 and 3.4. For $\beta=0.5, \mathrm{~L}_{\mathrm{r}}=53 \mathrm{~cm}$ and $\mathrm{L}_{l}=$ $160 \mathrm{~cm}$. For $\beta=0.65, \mathrm{~L}_{\mathrm{r}}=47 \mathrm{~cm}$. Therefore, $L=66.5$ and 66.3 for the two values of $\beta$. The maximum triton energy was $361 \mathrm{keV}$ and $152 \mathrm{keV}$ for $\beta=0.5$ and 0.65 , respectively. Since the triton injection energy is $97.5 \mathrm{keV}$, there will be some loss from the high-energy tail of the distribution. To see the effect this might have on the confined ion distribution, we can look at Fig. A-12 and A-13. A rather small part of the $\mathrm{T}^{+}$distribution will be cut off and virtually no added loss will result for the $\mathrm{D}^{+}$ions. The 3.52 $\mathrm{MeV} \mathrm{He}{ }^{++}$ions will be immediately lost uut the ends due to nonadiabaticity. If the machine were approximately 1.8 times larger or the field 1.8 times larger at $0.65 \beta$, then $\mathrm{He}^{++}$ions would be contained. Future work on determining adiabaticity should be done with ion trajectory calculations in a self-consistent magnetic field.

Figures A-14 and A-15 show the distribution of the ions escaping out the ends and are included here for comparison to the confined distribution shown in Figs. A-1 2 and A-I 3 and for future reference with respect to direct energy conversion of these end losses.

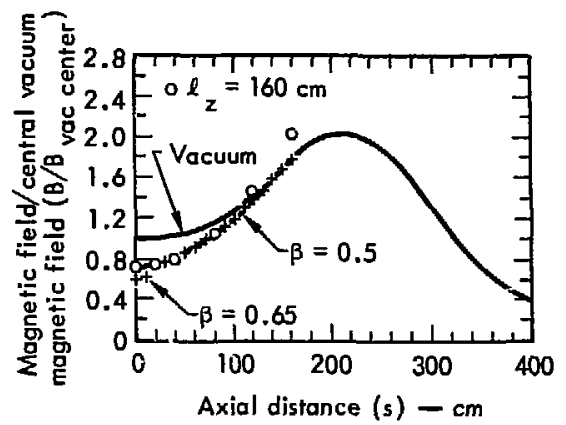

Fig. A-10. Analytic fit to the axial magnetic field for calculating the scale length for the adjabatic energy limit.

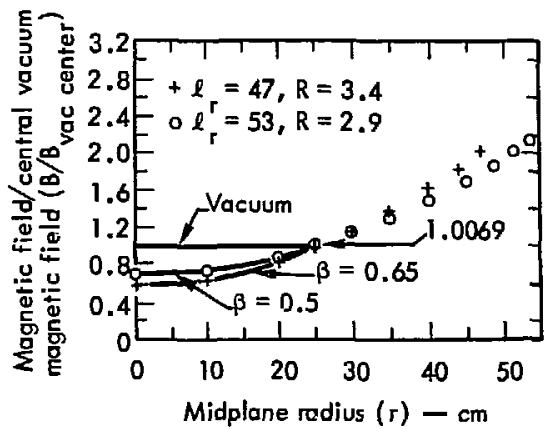

Fig. A-11. Analytic fit to the radial magnetic field for calculating the scale length for the adiabatic energy limit.

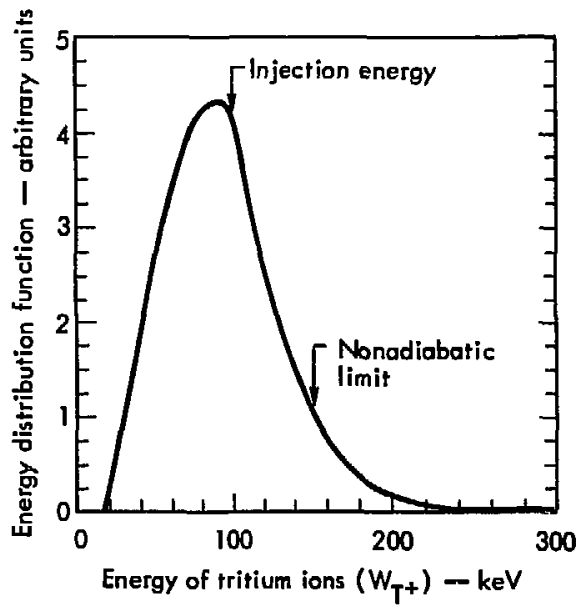

Fig. A-12. Contained tritium particle distribution versus tritium emergy. 


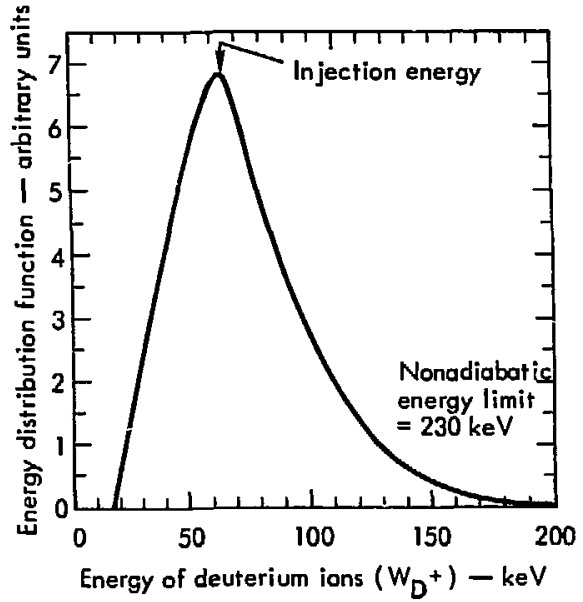

Fig. A-13. Contained deuterium particle distribution versus deuterium energy.

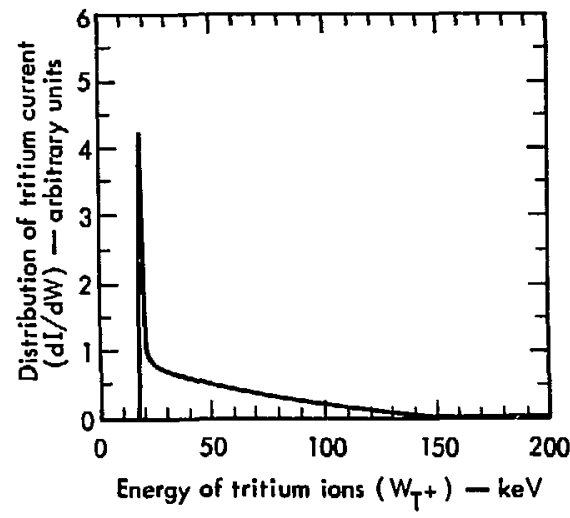

Fig. A-14. Distribution of tritium current versus energy leaking out the ends of the machine.

\section{STARTUP OF THE FERF PLASMA}

\section{Target Plasma Conditions}

We shall now derive approximate conditions which a target plasma must satisfy in order to start up the FERF plasma. We assume that at time zero the full $500 \mathrm{~A}$ of $D^{0}$ and $T^{0}$ are switched on and that a target plasma (just dense enough to attenuate neutral gas) has already been created which has an ion energy much less than 65-keV injection energy of deuterons

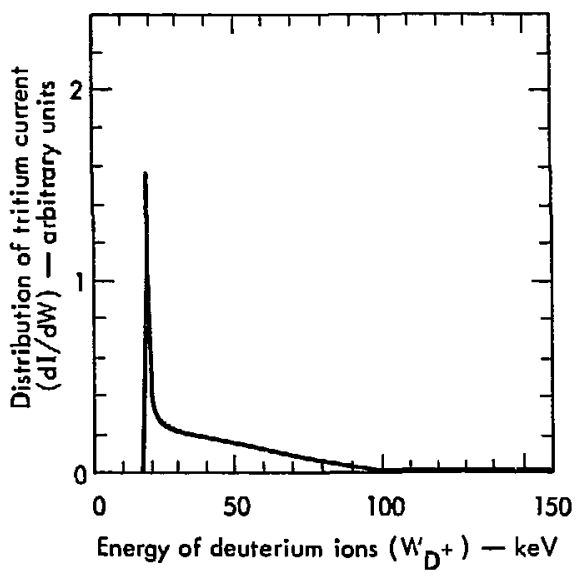

Fig. A.15. Distribution of deuterium current versus energy leaking out the ends of the machine.

(tritons have the same speed). The beam is assumed to be $20-\mathrm{cm}$ wide at the plasma and the target plasma diameter is also $20 \mathrm{~cm}$. The axinl-density profile for the target plasma is assumed to be the same as for the final steady-state hot plasma for the $\beta=0.5$ case (see Fig. A-1). Actually, the target $\beta$ is exceedingly small but tie density profile shape is very similar to the $\beta=0.5$ case.

The volume, $V$, of this plasma is given by

$$
\begin{aligned}
V & =\pi R^{2} L G^{(0)}=\pi \times 10^{-2} \times 420 \times 0.67 \\
& =88.4 \times 10^{3} \mathrm{~cm}^{3}
\end{aligned}
$$

The average density is less than the central density by the factor $\mathrm{G}^{\mathrm{l}} / \mathrm{G}^{0}=\mathbf{0 . 2 5 4}$.

At the end of a certain time called $\tau_{\mathrm{T}}$ (calculated below), we assume the target has decayed away according to $\mathrm{e}^{-\mathrm{t} / \tau_{T}} \mathrm{~T}$ and that it has been replaced by a hot plasma which is dense enough to attenuate (by one e-folding) the neutral gas in a distance of one gyrodjameter $\left(2 a_{i}\right)$. For $65-k e V$ deuterons in $3.75 \mathrm{~T}$, $2_{\mathrm{ai}}=2 .: 2 \mathrm{~cm}$. The requuired condition is

$$
\lambda=2 a_{i}=\frac{1}{n_{H}\left(\Sigma \sigma v / v D_{2}^{0}\right)}
$$


where $n_{H}$ is the density of hot plasma, $W \sim 65 \mathrm{keV}$, $\mathrm{V}_{\mathrm{D}_{2}}$ is the speed of incoming $\mathrm{D}_{2}$ molecules at $0^{\circ} \mathrm{C}$ $\left(v_{D_{2}} \sim 1.1 \times 10^{5} \mathrm{~cm} \mathrm{~s}^{-1}\right)$, and $\Sigma \sigma v$ are the reaction rates for ionizing incoming gas.

\section{A 50-eV-Electron Cold-lon Target Plasma}

We assume that the ions are sufficiently cold that the attenuation is only by electrons at $50-\mathrm{eV}$ mean energy.

$$
\begin{aligned}
& (\overline{\sigma v})_{\mathrm{ie}}=5.0 \times 10^{-8} \mathrm{~cm}^{3} \mathrm{~s}^{-1} \\
& \mathrm{n}_{\mathrm{H}}=\frac{1.1 \times 10^{5}}{2.72 \times 5 \times 10^{-8}}=0.8 \times 10^{12} \mathrm{~cm}^{-3}
\end{aligned}
$$

We assume that the plasma will build up from this $0.8 \times 10^{12} \mathrm{~cm}^{-3}$ density to the final steady-state density; however, whether or not the plasma will build up under FERF conditions is discussed later and is by no means assured. The electron dielectric constant $\left(\omega_{\mathrm{pe}} / \omega_{\mathrm{ee}}\right)^{2}$ for this plasma is 0.006 .

We will now calculate the time for the hot plasma to build up to $n_{\mathrm{H}}$, and this time will then set the allowable decay time for the target plasma. We assume that all $500 \mathrm{~A}$ hits the plasma but that the average cord length, $\ell$, is calculated to be only $63 \%$ of the diameter, or $12.6 \mathrm{~cm}$. The trapping rate is then $R_{t}$, so that

$$
\begin{aligned}
& R_{t}=\frac{I}{q}\left[1-\exp \left(-n_{T} \frac{\Sigma \sigma v}{v_{0}} l\right)\right] \\
& { }^{n} T={ }_{T} T_{0} e^{-t / \tau} T \\
& n_{H}=\frac{1}{v \frac{\bar{n}}{n_{0}}} \int_{0}^{T} T \frac{I}{q}\left[1-\exp \left(-n_{T} \frac{\Sigma \sigma v}{v_{0}} \ell\right)\right] d t
\end{aligned}
$$

The quantity $\tilde{n} / n_{0}$ accounts for the central density $n_{0}$ being greater than the average density.

If the beam is not strongly attenuated, the quantity in brackets can be expanded, giving

$$
n_{H}=\frac{I / q}{v \frac{\bar{n}}{n_{0}}} \frac{\Sigma \sigma v}{v_{0}} l \int_{0}^{\tau} n_{T_{0}} \tau_{T} \exp \left(-t / \tau_{T}\right) \frac{d t}{T_{T}}
$$

$$
=\frac{0.631 / \mathrm{q} \frac{\Sigma \sigma v}{v_{0}}}{v \frac{\bar{n}}{n_{0}}} n_{\mathrm{T}_{0}} \tau_{\mathrm{T}}
$$

$$
\begin{aligned}
\Sigma \sigma \mathrm{v}= & \sigma \mathrm{v}_{\mathrm{cx}}+\sigma \mathrm{v}_{\mathrm{ii}}+\sigma \mathrm{v}_{\mathrm{ie}} \\
= & 1.5 \times 10^{-16} \times 2.5 \times 10^{8}+1.0 \times 10^{-16} \times 2.5 \times 10^{8} \\
& +2.2 \times 10^{-8}=8.45 \times 10^{-8} ; \text { and } \\
\mathrm{n}_{\mathrm{H}}= & \frac{0.63 \times 500 \times 8.45 \times 10^{-8} 12.6 / 2.5 \times 10^{8}}{88.4 \times 10^{3} \times 0.254 \times 1.6 \times 10^{-19}} \\
& \times \mathrm{n}_{\mathrm{T}_{0}}{ }^{\tau} \mathrm{T}=373.4 \mathrm{n}_{\mathrm{T}_{0}}{ }^{\tau} \mathrm{T} .
\end{aligned}
$$

For the required value of $n_{H}$ for attenuation of neutral gas of $0.8 \times 10^{12} \mathrm{~cm}^{-3}$, the value of ${ }^{n} \mathrm{~T}_{0}{ }^{\top} \mathrm{T}$ which the target must satisfy is

$$
\mathrm{n}_{\mathrm{T}_{0}}{ }^{\top} \mathrm{T}=\frac{0.8 \times 10^{12}}{373.4}=2 \times 10^{9} \mathrm{~cm}^{-3} \mathrm{~s} .
$$

For ${ }^{n} T_{0}=2 \times 10^{13} \mathrm{~cm}^{-3}$, the target lifetime, $\tau_{\mathrm{T}}$, is $100 \mu \mathrm{s}$, which is well within the $2 \mathrm{X}$ plasma regime.

However, if for stability of the target plasma during build up, the electron dielectric constant is required to be unity $\left(\omega_{\mathrm{pe}}^{2} / \omega_{\mathrm{ce}}^{2}=1\right)$, then $\mathrm{n}_{\mathrm{H}}=1.9 \times 10^{14}$ $\mathrm{cm}^{-3}$, and $\mathrm{n}_{\mathrm{T}_{0}}{ }^{{ }^{\mathrm{T}} \mathrm{T}}=3.4 \times 10^{11} \mathrm{~cm}^{-3} \mathrm{~s}$ which is beyond present practice.

The total number of ions in the target plasma is $\mathrm{N}_{\mathrm{T}}$, where

$$
\begin{aligned}
\mathbf{N}_{\mathbf{T}} & =\bar{n} \mathrm{~V}=0.254 \times 2 \times 10^{13} \times 88.4 \times 10^{3} \\
& =4.5 \times 10^{17} \text { particies }
\end{aligned}
$$

We assumed that the quantity $n_{\mathrm{T}} \Sigma \sigma v \ell / v_{0}<<1$. In fact,

$\frac{\mathrm{n}_{\mathrm{T}}{ }^{\Sigma \sigma v \ell}}{\mathrm{v}_{0}}=\frac{2 \times 10^{13} \times 8.45 \times 10^{-8} 12.6}{2.5 \times 10^{8}}=0.085$

thereby justifying the assumption.

We conclude that a target of ${ }^{n} T_{0}{ }^{\top} T_{3}=2 \times 10^{9}$ $\mathrm{cm}^{-3} \mathrm{~s}$ which has a density of $2 \times 10^{13} \mathrm{~cm}^{-3}$ and a 
100- $\mu$ s decay time would be an adequate target plasma for startup purposes for attenuation of neutral gas. If $\left(\omega_{\mathrm{pe}} / \omega_{\mathrm{ee}}\right)^{2}=1$ is required for stability, then a more stringent requirement on the target will be needed. The target plasma requirements need much further study; fortunately, this topic is a major part of the BBII and $2 \mathrm{X}$ experimental programs as well as LLL's theoretical studies.

Now we will discuss the effect of gas impinging on the plasma during start-up. Start-up conditions differ from those of steady-state in that during start-up the fast neutrals impinging on the wall will bury about $1 \mu \mathrm{m}$ into the wall and take some time to diffuse back, whereas in steady-state the return is equal to the input. During the transient state, we will assume that the wall absorbs all but $10 \%$ of the incident beam. For restarting the piasma after prolonged operation, the walls would be saturated and other ussumptions would be called for to analyze the buildup. The other differen: : between startup and steady state is that the target plasma is much less dense and therefore is less efficient at burning out incoming gas.

We will assume that for the first few milliseconds no gas from the source will reach the plasma. Then, the trapping rate in the outer $2.72 \mathrm{~cm}$ (a layer one gyrodiameter thick) is given below for $6.5-\mathrm{keV}$ electron mean energy

$\operatorname{In}_{H} \frac{\Sigma \sigma v}{v_{0}} 2 a_{i}$

$500 \times 0.8 \times 10^{12} \frac{4 \times 10^{-8}+4.6 \times 10^{-8}+1.3 \times 10^{-8}}{2.5 \times 10^{8}}$

$\times 2.72=0.43 \mathrm{~A}$.

The loss rate from a discussion to follow is $I^{*}$.

$I^{*}=0.1 \frac{f w}{2} I \times f=0.1 \times \frac{0 . I}{2}-500 \times 0.7=1.75 \mathrm{~A}$.

From this simplified model, it appears that the plasma boundary would erode away if the hot density is $0.8 \times 10^{12} \mathrm{~cm}^{-3}$ at the plasma edge after the cold target disappears. However, if the hot density was four times more, or $3.3 \times 10^{12} \mathrm{~cm}^{-3}$, then buildup can be expected. Also, if the layer of plasma participating in ionizing incoming gas was thicker than a gyrodiameter due to noncoincidence of drift surfaces, then buildup would be more favorable.

\section{Kadial Density Profile and Charge-Exchange Losses}

Previously, we assumed the radial density profile to be a cubic, i.e., $N_{0}=N_{00}\left(1-(r / R)^{3}\right)$, and we have based our neutron fux and injection current requirements on this assumption. However, we have actually calculated the radial density profile using a computer code which uses cylindrical zones of thickness equal to a gyroradius of the mean energy deutrons. The density in each zone is assumed to be uniform radially, but to be linited in extent axially as shown in Fig. A-1. The outer zone of thickness \& will attenuate nearly all the thermal gas impinging on the plasma surface. The plasma ions and electrons can stop the neutral gas $\left(D_{2}^{0}\right)$ by ionization and by charge exchange. The chargeexchange process, however, results in a plasma ion being neutralized and lost unless it gets reionized by the plasma. This neutral particie will enter into the metal wall, diffuse back to the surface of the wall, and eventually reenter the plasma. The only way (since there is very little pumping) to remove the gas is by ionization. This process of the plasma pumping the gas by ionization tends to erode away the plasma surface. This erosion must be balanced by trapping the injected beam. For a stable gas/plasma interface, the following condition must be satisfied:

$$
\int_{0}^{\ell} \operatorname{In}\left[\frac{(\tilde{\sigma} \bar{v})_{i j}+(\bar{\sigma} \bar{v})_{i e}}{v_{0}}\right] d x \geqslant I^{*}
$$

where $I$ is the current of injected $D^{0}$ at speed $v_{0}$, and $\ell$ is the distance (at least 2 gyrc adii) over which ions can contribute to ionizing neutral gas. The left-hand side is the source of current feeding the plasma, and $I^{*}$ is the loss due to charge exchange.

There are then two distinct regions in the plasma: the outer layer of plasma with gas impingement and the core of the plasma with no gas. We will treat them separately. 


\section{The Plasma-Gas Boundary Layer}

We will now evaluate the left-hand side of $\mathrm{Eq}$. (A13). The injection current is $500 \mathrm{~A}$ of $65-\mathrm{keV} \mathrm{D}^{0}\left(\mathrm{v}_{0}=2.5\right.$ $\left.\times 10 \mathrm{~cm}^{3}-\mathrm{s}^{-1}\right)$. The cross sections are $(\bar{\sigma} \overline{\mathrm{v}})_{\mathrm{ie}}=4.7$ $\times 10^{-8} \mathrm{~cm}^{3} \cdot \mathrm{s}^{-1},(\overline{\sigma V})_{\mathrm{cx}}=4.2 \times 10^{-8} \mathrm{~cm}^{3} \mathrm{~s}^{-1}$, $(\overline{\sigma \mathrm{V}})_{\mathrm{ie}}=1.3 \times 10^{-8} \mathrm{~cm}^{3} \mathrm{~s}^{-1}$. The layer thickness or mixing length will be taken as four Larmor radii of $65-\mathrm{keV} \mathrm{D}^{+}$in $3.75 \mathrm{~T}$ or $5.0 \mathrm{~cm}$. The average density from the cubic profile is $0.77 \times 10^{14} \mathrm{~cm}^{-3}$ in the last $5 \mathrm{~cm}$ of the plasma. The source of the $\mathrm{D}^{+}$ions in this outer zone is

$500 \times 0.77 \times 10^{14}\left[\frac{\left(4.7 \times 10^{-8}+4.2 \times 10^{-8}+1.3 \times 10^{-8}\right)^{5}}{2.5 \times 10^{8}}\right]$

$$
=79 \mathrm{~A}
$$

The loss rate $\mathrm{I}^{*}$ due to charge exchange must not exceed $79 \mathrm{~A}$. To derive $\mathrm{I}^{*}$, we must follow the fate of a $\mathrm{D}_{2}$ molecule as it enters the plasma.

As it enters the plasma, the $\mathrm{D}_{2}$ molecule can be ionized or charge exchanged, which results in the loss of about one half a plasma ion to the wall since half goes outward and the other half goes inward and is reionized. The remaining $\mathrm{D}_{2}^{+}$can further ionize and be lost out the ends. We will not consider dissociation because at these energies it is a small effect.

The probability of charge exchange of $D_{2}$ is

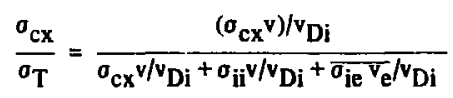

For one molecule entering the plasma, on the average $1 / 2 \sigma_{\mathrm{cx}} / \sigma_{\mathrm{T}}$ of a fast ion will be lost. That is, we assume that on tive average half the ions will head out and be lost and the other half will head in and be reionized.

The molecular ion can undergo charge exchange again and the number of fast ions lost is again $1 / 2 \sigma_{\mathrm{cx}} / \sigma_{\mathrm{T}}$; therefore, the fraction of fast ion lost per molecule in is

$$
f=\frac{\sigma_{C X}}{\sigma_{T}}
$$

However, each ion lost results in half a molecule retuming, which in turn loses more ions. Therefore, the total number of ions lost per external source $D_{2}$ is

$$
\begin{aligned}
& f+\frac{1}{2} f^{2}+\frac{1}{4} f^{3}+\frac{1}{2^{3}} f^{4}+=f\left(1+\frac{f}{2}+\frac{f^{2}}{2^{2}}+\frac{f^{3}}{2^{3}}+\right) \\
& =\frac{f}{1-\frac{f}{2}} .
\end{aligned}
$$

The loss current $l^{*}$ is then given as

$$
1 *=\frac{1^{0} f}{1-\frac{i}{2}}
$$

where $1^{0}$ is the current of $D_{2}$ from external sources such as steaming gas from the neutralizer and from charge exchange of plasma ions on the beam.

At $70 \mathrm{keV}$

$\sigma_{\mathrm{cx}}=3.3 \times 10^{-16} \mathrm{~cm}^{-2}, \sigma_{\mathrm{ii}}=2.0 \times 10^{-16}$

$(\overline{\text { ov }})_{\mathrm{ie}}\left(7 \mathrm{keV}=\overline{\mathrm{W}_{\mathrm{e}}}\right)=2.5 \times 10^{-8}, \mathrm{v}_{0}=2.6$

$\times 10^{8} \mathrm{~cm} \mathrm{~s}^{-1}$.

$\frac{\sigma_{\mathrm{cx}}}{{ }_{\sigma_{\mathrm{T}}}}=\frac{3.3 \times 10^{-16} 2.6 \times 10^{8}+}{3.3 \times 10^{-16} 2.6 \times 10^{8}+2.0 \times 10^{-16} 2.6 \times 10^{8}+2.5 \times 10^{-8}}$

$=0.527$

$f=0.53$

$I^{*}=1^{0} \frac{0.53}{1-0.53 / 2}=0.721^{0}$.

This means that it takes $\mathbf{0 . 7 2}$ ions to destroy each molecule that originates from an external source.

From Appendix $\mathrm{C}$, we see that $\mathrm{l}^{\mathrm{O}}$ is made up of $I_{\text {ext }}+\frac{f_{w}}{2}-$ 
where $f_{w}$ is the fraction of injected atoms which via charge exchange hit the wall, and at $70 \mathrm{keV}$.

$$
f_{w}=0.12 \text { for } \gamma=4 \text {. }
$$

The loss current is then

$$
I^{*}=0.72 \mathrm{I}_{\text {ext }}+\frac{0.12}{2} 0.72 \mathrm{I} .
$$

For no external source and $500 \mathrm{~A}$ oi $\mathrm{D}^{0}, \mathrm{I}^{*}=22 \mathrm{~A}$. We have allowed for $10 \mathrm{~A}$ of $\mathrm{D}_{2}$ to stream into the plasma along the beam lines and $10 \mathrm{~A}$ to back-stream into the plasma from the end regions. Thus, $I^{*}$ might be as much as $\mathbf{3 6} \mathrm{A}$. This simplified analysis indicates the plasma can maintain itself against the erosion effect of the incident gas. Much more refined studies of this plasma/gas boundary layer will be needed.

The computer code has the above assumption built in and many more refinements; however, for highdensity cases of interest here, nearly all of the gas is stopped in the first zone so that all succeeding zones do not have so many processes going on. The code does take the following processes into account:

- lons trapped in one zone spend some time in adjacent zones due to the finite gyroradius and mixing of ions from drift surfaces being split as in banana surfaces in tori. The zone thickness is taken as a Larmor diameter at the plasma edge.

- Diffusion from zone to zone due to coulomb collisions.

- Axial loss due to collisional scattering into the loss cone.

- Attenuation of the injected beam.

- Generation and reionizstion of fast neutrals resulting from charge exchange of plasma ions on the neutral beam. Of these neutrals, $99 \%$ are going in the beam direction rather than in the backward direction.

\section{Buildup from Initial Target to Finai State}

In the previous section, the conditions were given for a target plasma which would be replaced by a beam-fed plasma by the time the target had decayed. The conditions were also given for this plasma to grow in radjus as well as density. As the plasma grows, it also charge exchanges on the beam, the result being that neutrol atoms impinge on the walls. Fortunately. during buildup (which requires only a few 10's of milliseconds), the neutrals which bombard the wall do not have time to come into diffusion equilibrium and back out of the metal wall. After some time however, the wall will saturate and each atom entering will also return. The main questions then are

- Will the plasma spring up from the target?

- Will the radial density profile be roughly a cubic as previously assumed?

- After a long time, will the plasma be able to ionize the incoming gas without its surface eroding away?

To address these questions, a radial-buildup computer code wass written to calculate time-and spacedependent evolution of the plasma density, attenuation of the injected neutral beam, and attenuation of the gas streaming into the plasma. The plasma was modeled by a set of concentric shells of constant density. The thickness of each shell was taken to be one gyrodiameter of the mean energy ion. The injection process due to the finite orbit size compared to a zone thickness resulted in an ion being ionized in one zuis spending some time in the two adjacent zones. This was :he main radial growth mechanism. Also, collisional diffusion from one zone to the nex: was included and contributed to radial growth. The main loss mechanisms were coulomb scattering into the loss cone and charge-exchange loss.

Severnl example problems were run on the code to answer the above questions. The first case had a 500-A, 16-cm-wide beam of $\mathrm{D}^{0}$ injected perpendicularly to the field lines at the midplane. One ampere equivalent of $D_{2}^{0}$ gas was assumed to be streaming in along the beam line; $99 \mathrm{~A}$ of $\mathrm{D}^{0}$ atoms resulting from charge exchange hit the walls. For this problem, the wall was assumed to be saturated so that $49 A$ of $D_{2}^{0}$ returned to the plasma. The unattenuated portion of the beam was the $1 A$ of $D^{0}$ which impinged on the opposite wall. This equilibrium distribution was established in $17 \mathrm{~ms}$. The radial profile is shown in Fig. A-16. Two features should be noted: the radial profile is reasonably closely approximated by a cubic profile ( as was assumed in computing the neutron flux ; and the beam fills the center even though the beam is attenuated by $\mathrm{e}^{-6}$ across the plasma. One surm: wht worrisome feature of Fig. A-I is the way the densiry peaks at about a $7-\mathrm{cm}$ radius. The interchange 


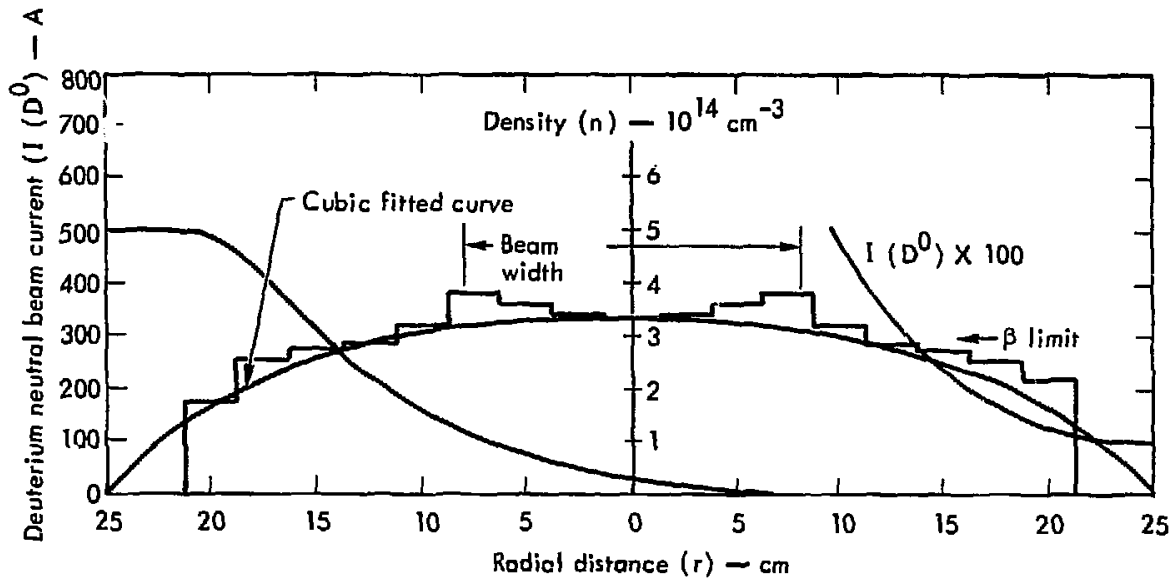

Fig. A-16. Stes:lv-state radial density profile and attenuated neutral beam. Note the cubic analytic fit to the calculated density profile.

instability might transport plasma inward as well as causing enhanced plasma losses outward and axially. The $\beta$ there would exceed the critica: $\beta$ for the mirror mode. If enhanced losses would occur locally, the density might saturate at a value corresponding to the critical $\beta$.

The second case was similar to the previous one except $10 \mathrm{~A}$ of $\mathrm{D}_{2}^{0}$ gas was included and an initial target at $3 \times 10^{14} \mathrm{~cm}^{-3}$ Jensity and $5-\mathrm{cm}$ radius was imposed. The beam was switched on at time zero with $400 \mathrm{~A}$ of $65-\mathrm{keV} \mathrm{D}^{0}$. The evolution is shown in Fig. A-17. Rather than absorbing nettrals, the walls were assumed to be saturated. In spite of this the plasma did grow to its steady-state profile. Significant off-axis density peaking occurred during the buildup. Another run (not shown) was made with $50 \mathrm{~A}$ of streaming $\mathrm{D}_{2}^{0}$ : buildup occurred.

To illustrate how the gas erodes the edge of the plasma, a case was run with a $20-\mathrm{cm}$ plasma target at $3 \times 10^{14} \mathrm{~cm}^{-3}$, but with $80 \mathrm{~A}$ of $\mathrm{D}_{2}^{0}$ gas. The edges of the plasma did indeed shrink. and at the same time the density peaked. These results are shown in Fig. A-18.

Finally, a case very close to the FERF design was run at $500 \mathrm{~A}$ of $\mathrm{D}^{0}$ with $34 \mathrm{~A}$ of $\mathrm{D}_{2}^{0}$ impinging on the plasma (Fig. A-19). The density was generally lower than for a cubic profie and some off-axis peaking did occur.

Much more work is naeded on buildup calculations, especially since the FERF design is at such a low energy that the plasma's ability to bum out incoming gas is near its limit. Also, the exact limit (discussed earlier) depends sensitively on the mixing-length concept, one which is not very well understood quantitatively. 


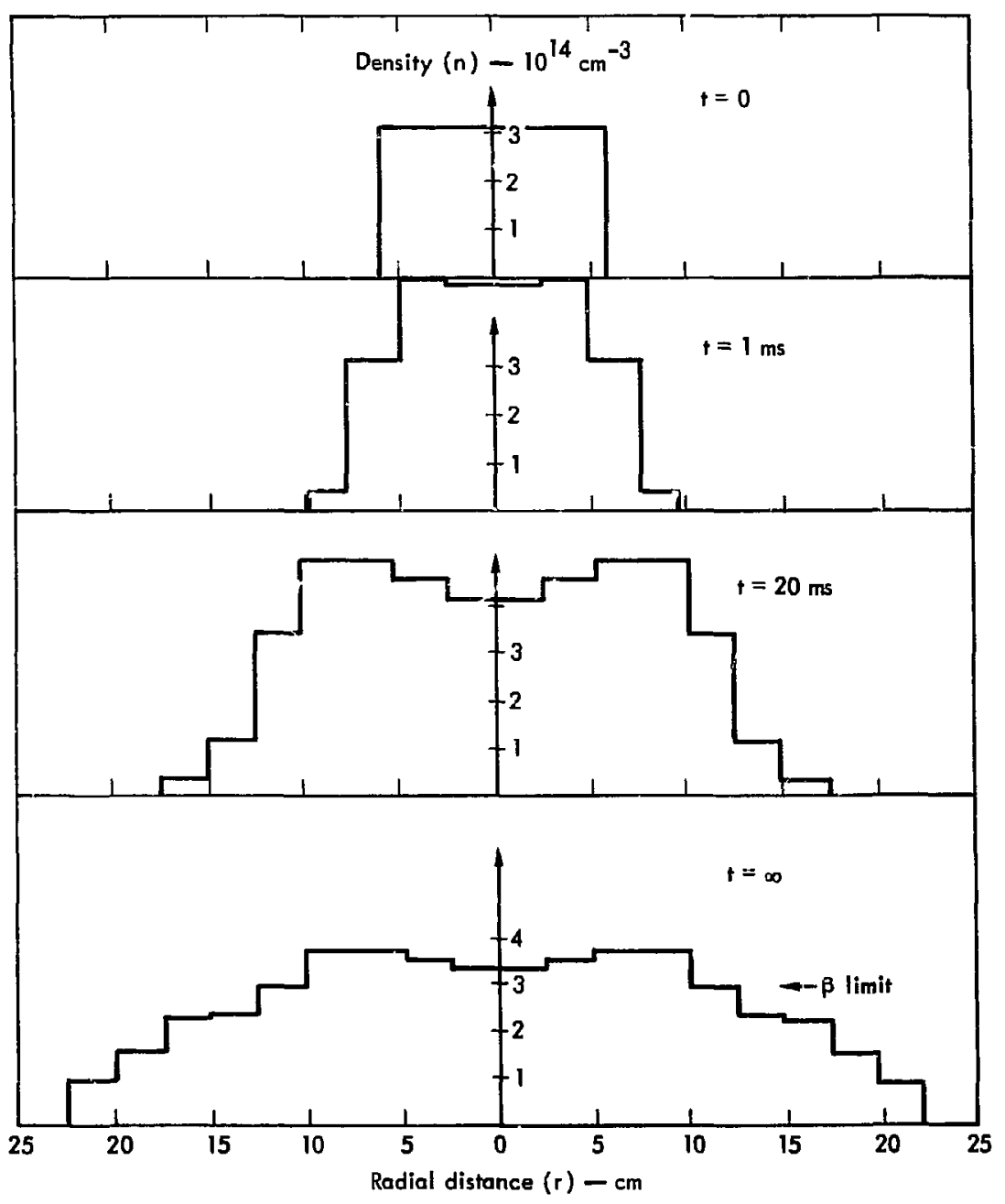

Fig. A-17. Time sequence of radial density profile during buildup showing radial growth. 


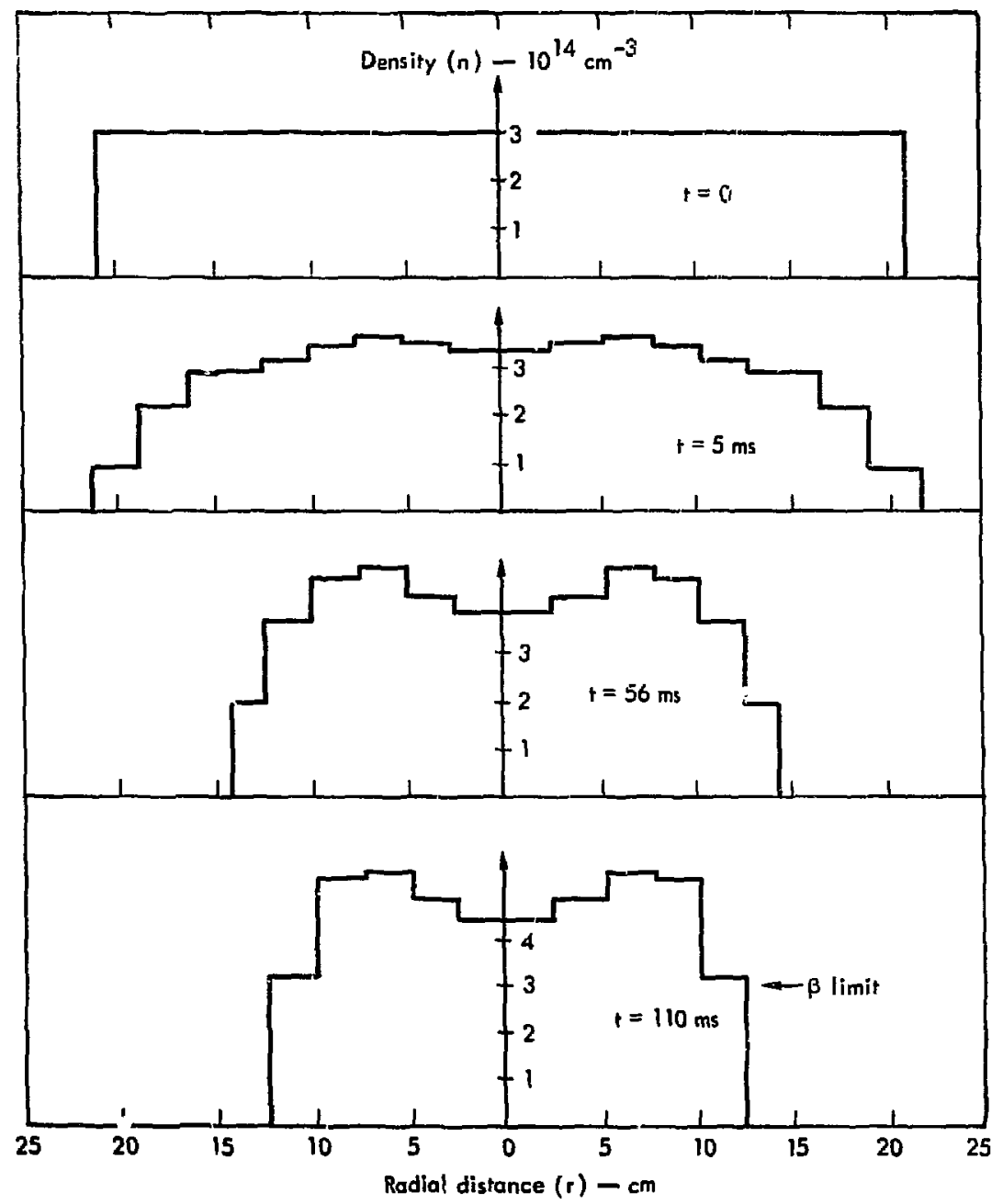

Fig. A-18. Time sequence of radial density profile showing the effect of the neutral gas croding away the plasma edge. 


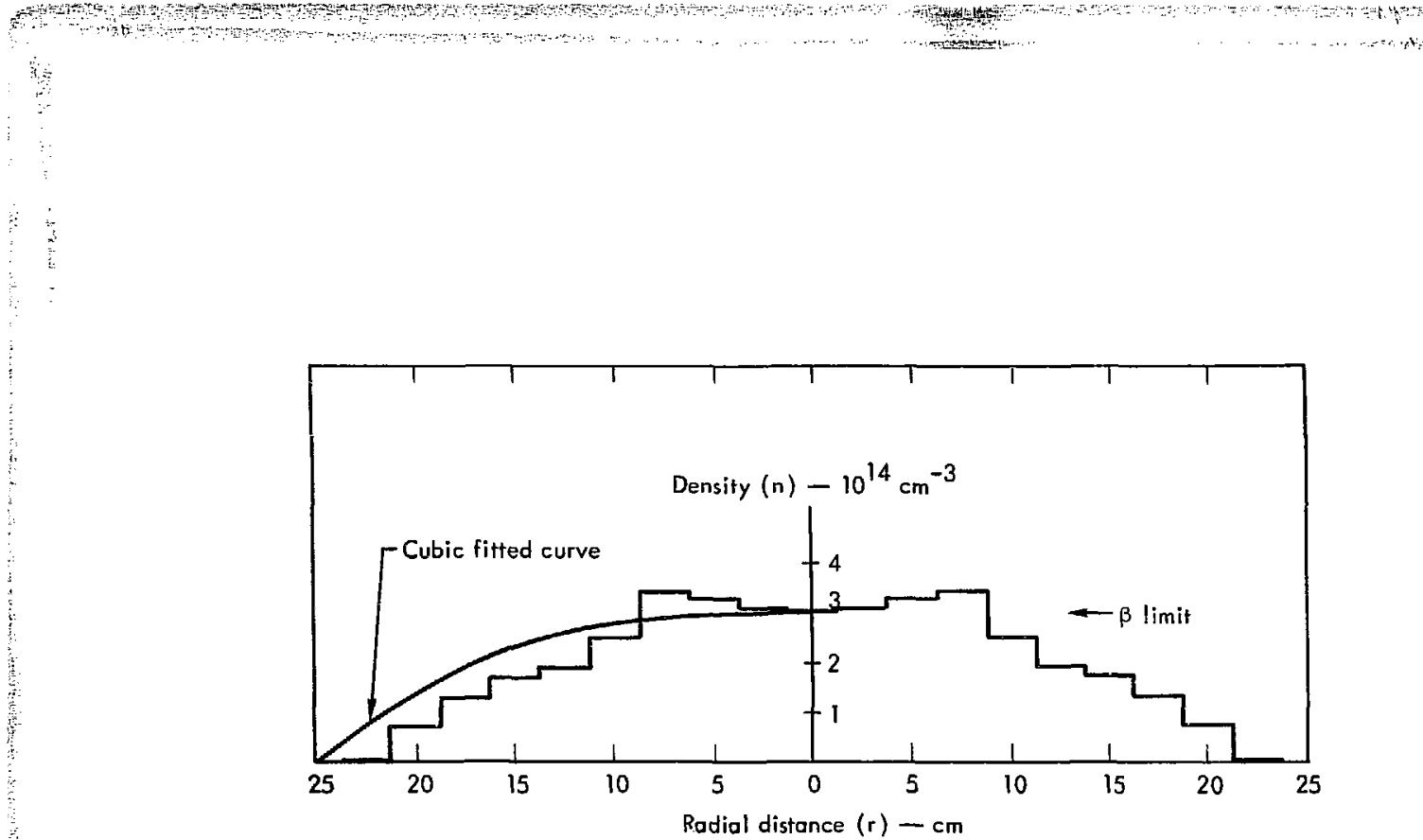

Fig. A-19. Steady-state ıddial density profile.

\section{REFERENCES}

A1. D. E. Baldwin and J. D. Callen, Phys. Rev. Letters 28, 1686 (1972).

A2. J. H. Foote, Plasma Phys. 14, 543 (1972). 


\section{Appendix B - Neutral Beam Injectors}

\section{SELECTION OF INJECTOR TYPE}

The principal factors determining the selection of an injector for a fusion reactor are the required energy and current of the injected beam; the tolerable gas load upon the trapped plasma; the beam access; the available beam densities; and the efficiencies of beam acceleration, neutralization, and trapping. The choice of primary ion species (atomic or molecular, positive or negative) is governed by the efficiency of conversion to neutral particles at the required energy and by the relative difficulty of ion production.

Available densities of the primary beam are determined by technological limitations and by fundamental limitations in the ion source and in the accelerator structure. Averaged over large extraction areas, these limitations are currently as follows:

- Available source densities are now about 300 $\mathrm{mA} / \mathrm{cm}^{2}$ for positive atomic ions $\left(\mathrm{D}^{+}\right.$and $\left.\mathrm{T}^{+}\right) ; 100$ $\mathrm{mA} / \mathrm{cm}^{2}$ for molecular ions $\left(\mathrm{D}_{2}^{+}\right.$and $\mathrm{D}_{3}^{+}$); and $12 \mathrm{~mA} / \mathrm{cm}^{2}$ for negative ions ( $\mathrm{D}^{-}$or $\mathrm{T}^{-}$).

- Limitations on beam power within the highvoltage accelerating structure are due to electrode heating, back-streaming electrons, $x$ rays, gas evolution, and related problems. Power densities of about $4 \mathrm{~kW} / \mathrm{cm}^{2}$ have been achieved in continuously operated systems. Power densitit:s are limited by electrode warpage or melting: this limitation (which will apply to the FERF injectors) will probably be eased but not overcome by improvements such as better optics and better cooling to protect the electrodes from the beam. However, such improvements do not appear essential to fulfilling the FERF injection requirements. Of course, higher densities can be produced by pulsed systems. $B$

- These beam densities apply to the primary beam produced by the ion source and accelerator. They are reduced by the efficiencies of the neutralizer ( $50 \%$ to $75 \%$ ) and of trapping in the reactor (40\% to 95\%). Also, the beam extraction area may be as small as $15 \%$ of the total source assembly area, the desired ion specie $\left(\mathrm{D}^{+}, \mathrm{D}_{2}^{+}\right.$, or $\left.\mathrm{D}_{3}^{+}\right)$will probably constitute no more than $70 \%$ of the total beam, and access of the beam to the reactor is restricted by magnet coils, neutron shielding, and other require- ments. For these reasons, and also to reduce the gas flow (proportional to beam area) from the injection system into the main chamber, the density of the injected beam must be high $\left(0.4\right.$ to $\left.2.0 \mathrm{~A} / \mathrm{cm}^{2}\right)$. Therefore. it is necessary to increase the beam density by a factor of 50 to 300 .

The beam density maybe increased either by a system of converging neutral beams or by a magnetic guide field to guide charged ions into the reactor. In one way or the other, the system must act as a funnel to concentrate the beant. The magnetic guide field ${ }^{\mathrm{B}} 2$ is usable with negative ions or molecular ions but is not usablc with positive atomic ions $\left(\mathrm{D}^{+}\right.$or $\mathrm{T}^{+}$).

Riviere $^{\mathrm{B} 3}$ has surveyed the neutralizing efficiencies for all possible $D$ and $T$ ion species as a functicn of energy. From his data (Fig. A3.8 in Ref. B3), it can be seen that for $D^{0}$ energies below $100 \mathrm{keV}$, the highest power efficiency for production of neutrals is attainable by charge exchange of $\mathrm{D}^{+}$in a gas neutralizer. This efficiency (for $D^{+} \rightarrow D^{0}$ ) drops rapidly with increased energy, while the power efficiency for neutral production from negative ions $\left(\mathrm{D}^{-} \rightarrow \mathrm{D}^{0}\right)$ is inscnsitive to energy. The result is that these two processes are competitive at $100 \mathrm{keV}_{\text {, but that }} \mathrm{D}^{+}$is preferred at lower energies because of the greater ease of $\mathrm{D}^{+}$ production. For the energy of optimum neutron yield (about $65 \mathrm{keV}$ ) the $\mathrm{D}^{+}$neutralization efficjency in $\mathrm{D}_{2}$ gas is $68 \%$ for equilibrium cell thickness.

Much information is available on the use of various gases, vapors, and thin foils as neutralizing targets. The best results consistent with FERF requirements have been attained in the $2 \mathrm{XII}^{\mathrm{B} 4}$ and Ormak ${ }^{\mathrm{BS}}$ experiments using hydrogen or deuterium gas in a neutralizing cell closely coupled with the ion source. In this configuration, the neutralizing gas does not perturb the ion source and charged-beam blow-up due to a residual space charge is avoided. However, this technique is suitable only if excitation of the atomic neutral beam is not required and if means can be found for pumping the large quantity of hydrogen gas.

Preliminary designs have been considered for two types of neutral injection systems (using $\mathrm{D}^{+}$and using $\mathrm{D}^{-}$as the primary beam) and also for a $\mathrm{D}^{-}$injection system using a magnetic gujide field. The $D^{+} \rightarrow D^{0}$ neutral injection system (similar to the $2 \mathrm{XII}$ and 
Ormak systems) was chosen for FERF because it is most favorable for energies under $100 \mathrm{keV}$ and because it requires the least amount of new development. This choice is subject to several assumptions, each of which will be examined in this report:

- The $65-\mathrm{keV}$ of $D^{0}$ energy is optimum for neutron production, but must be proven acceptable with respect to bombardment of the first wall of the reactor by neutrals produced by charge exchange of the neutral beam penetrating the reactor plasma. The beam energy must also be proven suitable for beam penetration and buildup of a trapped plasma of the desired diameter, density, and spatial distribution.

- lon sources and acceleration systems suitable for continuous operation at voltages of 65- to 100 $\mathrm{keV}$ must be developed. In this respect, the FERF requirements represent an advance over high-current, pulsed systems now in operation at lower voltages.

- The system must operate in a radiation environment of at least $10^{11} 14-\mathrm{MeV} \mathrm{n} / \mathrm{cm}^{2}$ 's in addition to radiation by slower neutrons, gamma rays, and $\mathrm{x}$ rays and contamination by tritium. The sources must operate continuously for very long periods ( 6 months, perhaps) without maintenance because of the difficulties imposed by neutron-induced radioactivity and by tritium contamination. Also, the possibility of insulator damage by neutrons must be examined.

\section{INJECTOR LAYOUT}

The yin-yang magnet provides the opportunity to install four wide-angle injection beam lines between the two coils. These wide-angle beam lines, two of which are snown by Fig. 1 (page 5 ) are well suited for converging neutral beams entering the magnetic field at an angle almost perpendicular to the flux lines. Therefore, the trapping will be almost $100 \%$ if the plasma density is sufficiently high, since none of the ionized particles will be in the loss cone. Beam exits will not be possible for these wide-angle beams. However, after passing through the main chamber the beams will be sufficiently well spread out for the cooled walls to accept the beam power even if there is no attenuation by the trapped plasma.

Additional beam entrances and exits are available near the $x$ and $y$ magnet axes, as in the case of the
Baseball and 2 XII beam paths. However, these are not well adapted for neutral beams converging from a very broad source array and therefore will not be relied upon for the inain injection system. The access paths along the $x$ and $y$ axes will be useful for irradiation of large samples, plasma diagnostics, and perhaps for reactor startup.

The layout indicated by Fig. 1 (page ) provides a $20-\mathrm{cm}^{2}$ entrance window for each of the four beam lines. At a distance of about $4 \mathrm{~m}$ from the ion source extractors, the entrance window defines an acceptance angle of $\theta= \pm 1.4$ degrees. At $65 \mathrm{keV}$, the maximum transverse particle energy acceptable by this geometry is

$65 \mathrm{keV}(1.4 \text { degree } / 57.3 \text { degree })^{2}=20 \mathrm{eV}$.

The $20 \mathrm{eV}$ transverse energy acceptable by this geometry may be compared with a 7.4eV Gaussian transverse energy measured on a $16-\mathrm{keV}$ beam at $\mathrm{LBL}^{\mathrm{B} 6}$ and a 8- to 10-eV Gaussian transverse energy measured on a $1-\mathrm{keV}$ beam at $\mathrm{LLL}^{\mathrm{B} 7}$. These transverse energies represent the effects of source plasma temperature, optical aberrations, and minimal scattering and blowup in the neutralizer.

If the transverse energy in the FERF beam is $10 \mathrm{eV}$, the Gaussian l/e divergence angle will be

$$
\bar{\theta}=(10 / 65,000)^{1 / 2}= \pm 0.7 \text { degrees } .
$$

The fraction of the neutral beam lost at the entrance window will be

$$
\exp -(\theta / \bar{\theta})^{2}=0.02
$$

Eqch of the four injector assemblies consists of an array of ion source modules approximately $2 \times 2 \mathrm{~m}$ in overall dimensions. Using plasma sources of the dimensions of the Berkeley 50-A source, the source array will consist of a maximum of 28 modules arranged in " $X 4$ matrix as shown by Fig. B-1. The subdivision "to $\mathrm{D}^{+}$and $\mathrm{T}^{+}$sections can be adjusted.

The injer $r$ layout now being described is designed for a nomin neutral beam requirement of about $500 \mathrm{~A}$ at $65 \mathrm{keV}$ of $D^{0}$ energy and $100 \mathrm{keV}$ of $T^{0}$ energy. This beam requirement includes a factor of three to allow for enhanced scattering losses. To inject $500 \mathrm{~A}$ 


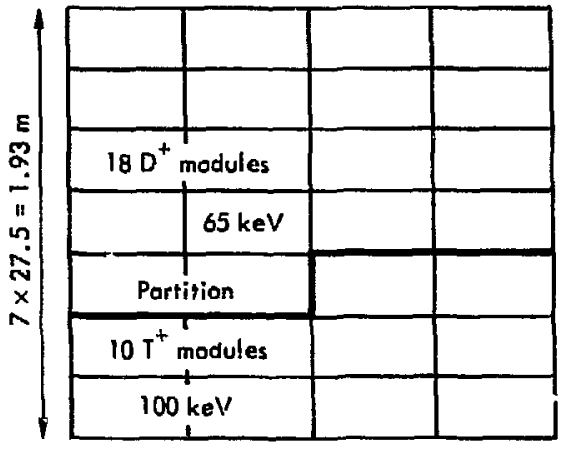

Aligned with direction of FERF fringing field

Fig. B-1. Lyyout of 28 source modules for each injector assembly. The subuivision into $\mathrm{D}^{+}$and $\mathbf{T}^{+}$sections can be adjusted to optimize this ratio.

of $\mathrm{D}^{0}$ and $\mathrm{T}^{0}$ into the reactor plasma at full energy, the sources must produce about $1200 \mathrm{~A}$ of primary ions in order to allow for the $67 \%$ neutralizer efficiency and $30 \%$ bearn losses. The latter has two causes: $10 \%$ to $25 \%$ of the primary beam will probably consist of molecular ions; and $5 \%$ to $10 \%$ of the beam will be last by collisions with duct walls, collimators, and background gas. The molecular ions will be included in computations of the power load and the gas loads, but will be excluded from computations of the neutron yield because the half-energy trapped ions derived from molecular ions will be rapidly scattered out of the reacting plasma.

The beam energies ( $65 \mathrm{keV}$ for $\mathrm{D}^{0}$ and $\mathrm{i} 00 \mathrm{keV}$ for $T^{0}$ ) have been chosen to maximize the neutron flux from a high $-\beta$, mirror-contained plasma and to equalize the speeds of the injected neutrals. Therefore, the neutralization efficiencies and the beam penetrations for $D^{0}$ and $T^{0}$ will be equal. To equalize the trapped densities of $\mathrm{D}^{+}$and $\mathrm{T}^{+}$and compensate for the effects of the ion mass and the ion energy upon the trapped lifetime, the injected beams should consist of approximately $364 A$ of $D^{0}$ and $136 A$ of $T^{0}$.

The injector assemblies will be designed to fulfill the nominal requirement for a 500-A injected beam by using an average of only 3.5 of the four beam lines.
This will purmit shutting down ont beam line at a time to recycle the cryogenit pumps. The time-averaged injected bean will exceed the requirement if all four assemblies are operaled part of the time, since the recycling of the cryogenic pumps will occupy a smill fraction of the total operating time.

In Table B-I, the injected beam from four source assemblies of $\mathbf{2 8}$ modules is computed under the assumptions described above. It may be convenient (depending upon buildup calculations) to subdivide the 28 modules into $18 \mathrm{D}^{+}$source modules and $10 \mathrm{~T}^{+}$ modules. us slıown by Fig. B-1. The source module specifications are basud upon desigr considerations which will be explained later.

We must also consider the possibility that injection requirements may be upgraded at a later stage of FERF design because of increases in magnetic field strength, plasma volume, plasma loss rates, or neutron flux requirements. At this time, we shall indicate the respects in which the injector design may be upgraded, but shall not carry out the upgraded design in detail.

- The bear donsity limitation of $4 \mathrm{~kW} / \mathrm{cm}^{2}$ is technological, not fundamental. We expect that this limitation will be raised by technological improvements, but it is difficult to predict the ultinate limitation.

- The source module design now presented provides for a beam aren of only $210 \mathrm{~cm}^{2}$, although the overall module size is $1500 \mathrm{~cm}^{2}$. Most of the module area is occupied by insulators, magnetic shielding, eiectrode mountings, and duct walls. The overall area could be more effectively utilized if the module size were increased, since the size of the insulators, shielding, and mountings do not scale directly with module size.

- It may be possible to increase somewhat the area of the injector array by lengthening the array in the direction perpendicular to the view shown by Fig. 1 (page 5 ). This increase would result in a nonrectangular array and probably would require relocation of the magnet structure and an increase of the window arca at the entrance to the main chamber.

- The requirements for beam power and gas pumping will scale directly with the upgraded beam requirements. A large fraction of the gas load on the reactor plasma will also scale directly with the increased beam; however, the diffuse gas and streaming gas from the 
Tatle B-1. Injected-beam parameters.

\begin{tabular}{|c|c|c|c|}
\hline \multicolumn{4}{|l|}{ Source module specifications: } \\
\hline Overall module size & \multirow{3}{*}{\multicolumn{3}{|c|}{$\begin{array}{l}27.5 \times 52.5 \mathrm{~cm}=1444 \mathrm{~cm}^{2} \\
3 \times 2 \times 35 \mathrm{~cm}=210 \mathrm{~cm}^{2} \\
4 \mathrm{~kW} / \mathrm{cm}^{2}\end{array}$}} \\
\hline Ion extraction area & & & \\
\hline Beam power density & & & \\
\hline Source module: & Deuterium & Tritium & Total \\
\hline Beam energy (keV) & 65 & 100 & \\
\hline Beam current density $\left(\mathrm{mA} / \mathrm{cm}^{2}\right.$ ) & 60 & 40 & \\
\hline Beam current per module (A) & 12.6 & 8.4 & \\
\hline \multicolumn{4}{|l|}{ Total beam from 4 injector assemblies (A) } \\
\hline Number of modules & 72 & 40 & 112 \\
\hline Total beam current $(A)$ & 907 & 336 & 1,243 \\
\hline Neutral beam produced at $67 \%$ conversion efficiency & 605 & 2.24 & 829 \\
\hline Charged beam to be dumped at first pumping stage (A) & 302 & 112 & 414 \\
\hline $\begin{array}{l}\text { Neutral beam losses ( } 30 \% \text { ) due to molecular ions, } \\
\text { background gas, collimation, etc. }\end{array}$ & 182 & 67 & 249 \\
\hline Injected neutral beam from four injector assemblies & 423 & 157 & 580 \\
\hline Time-averaged injected beam if average usage is 3.5 assemblies & 370 & 137 & 507 \\
\hline Nominal injection requirement & 364 & 136 & 500 \\
\hline
\end{tabular}

neutralizers will depend only upon neutralizer area and not upon beam density.

\section{GAS PUMPING REQUIREMENTS}

Consideration of the requirements for pumping the source gas and neutralizer gas must precede detailed design of the ion source and neutralizer modules. The required gas flow rates and pressures are based upon source gas flow rates, neutralizer pressures and conductance, and beam-line pressure and pumping speed.

\section{Source-Gas Flow Rates}

The total ion beam produced by each injector assembly of 28 modules is $311 \mathrm{~A}$. Source-gas efficiencies (the ratio of extracted ions to gas molecules fed to the source) are ty pically $\mathbf{3 0 \%}$, which implies a gas-feed rate of 1,040 equivalent amperes. Since the beam is mostly monatoric and the gas molecules $\left(D_{2}\right.$ and $T_{2}$ ) are diatomic, the gas flow into the neutralizer assembly is about $880 \mathrm{~A}$. The pumping requirement for each injector assembly is $922 \mathrm{~A}$, including the neutralizer gas and the gas produced by dumping the residual charged beam. This is recapitulated in Table B-2.

\section{Neutralizer Pressures}

The neutralizer dimensions and pressure will be adjusted to provide adequate gas thickness for neutralization of the beam by electron capture collisions, but the pressure will be limited to $10^{-3}$ Torr to avoid highvoltage breakdowns and premature electron capture during the acceleration. To attain an efficiency near the maximum the neutralizer thickness $\pi$ should be about $0.8 \times 10^{16}$ molecules $/ \mathrm{cm}^{2}$. A satisfactory neutralizer design, shown by Fig. 13 (see page 14 ), consists of a cooled, $60-\mathrm{cm}$-long, magneticallyshielded duct at a uniform gas pressure determined by the gas impedance of vanes at the output end. The desired thickness is attained at $10^{-3}$ Torr by cooling the warm gas by collisions with the cold wall. 
Table B-2. Gas-pumping requirements.

Gas to be pumped, each beam line:

From neutralizers: 880 A, diatomic

From charged beam:

103 A, monatomic

Less streaming gas and

diffuse gas to main

chamber:

$10 \mathrm{~A}$, diatomic

Total pumping load:

$922 \mathrm{~A}=180$ Torr $\cdot \ell / \mathrm{s}$

Gas to be pumped at first stage

(pressure $=3.7 \times 10^{-4}$ ) Torr: 166 Torr $\cdot \ell / \mathrm{sec}$

Required pumping speed: $4.5 \times 10^{5} \mathrm{l} / \mathrm{s}$

Gas to be pumped at second stage (pressure $=3 \times 10^{-5}$ Torr): 14 Turr $\cdot \mathrm{l} / \mathrm{s}$

Required pumping speed: $4.5 \times 10^{5} \mathrm{l} / \mathrm{s}$

In Appendix I, it will be shown that the required pumping speed can be attained by $5-\mathrm{m}^{2}$ cryog panels for each of the two pumping stages.

\section{PLASMA SOURCE MODULES}

The layout of the plasma source modules is based upon the 50-A modules developed at $\mathrm{LBL}^{\mathrm{B} 9}$ for pulsed injection into $2 X I I$. However, they will operate at a lower current density because of the higher voltage and continuous operation required for FERF. Larger modules being developed at LLL will probably be preferable. The extraction area of the 50-A source is $7 \times 35 \mathrm{~cm}$; however, probe measurements indicate that adequate plasma for extraction is produced over a larger area. We expect that the usable extraction area will be reduced by the need for cooling circuits within the grid. To make ample allowance for cooling and rigidity of the grids, we shall design for only $210-\mathrm{cm}^{2}$ extraction area although the plasma area may be $350 \mathrm{~cm}^{2}$ per module. Such a layout is shown by Fig. II (see page 12 ).

With an extracted bearn density of $60 \mathrm{~mA} / \mathrm{cm}^{2}$, the plasma source must be designed for at least $21 \mathrm{~A}$ of extracted beam. In comparison with other LBL plasma sources (those not using magnetic fields), this implies an arc current requirement of about $2100 \mathrm{~A}$ and an are voltage of about $50 \mathrm{~V}$. To obtain the required stability and uniformity of the arc discharge, the cathodes must be designed to emit the $2100 \mathrm{~A}$ thermionically, without relying on other emission processes which are subject to fluctuation.

We have studied a cathode design for emission of 2100 A from tungster filaments at $2700 \mathrm{~K}$ with a design lifetime of at least 6 months $(4,000 \mathrm{~h})$. Hot tungsten was chosen for the cathode material because of its predictable performance and lifetime (the latter being limited only by evaporation). However, this choice is probably not optimum because of the high temperature and high power requirement $(120 \mathrm{~kW} / \mathrm{module})$ required to attain the emission from a tungsten surface. A large economy will be possible if low-temperature cathodes, perhaps oxide-coated, can be proven satisfactory for the FERF requirements. Further testing and development will be required to ascertain whether oxide or thin-film cathodes will adequately resist the effects of ion bombardment and poisoning under FERF conditions.

It is most convenient to heat the filaments ohmically, using low-yoltage three-phase, $400-\mathrm{Hz}$ a.c. heating power. A simple filament mounting, shown by Fig. 11 (see page 12 ), consists of four filament conductors in parallel connected to form each of the three sides of a delta-connected filament circuit.

The LBL sources typically have gas efficiencies of $25 \%$ to $35 \%$, where we are defining the gas efficiency as the ratio of extracted beam ions (mostly $D^{+}$) to gas molecules $\left(D_{2}\right)$ fed to the source. Therefore, we are using a gas efficiency of $30 \%$ to compute pressures and pumping requirements in the neutralizer and in the beam line. Although conditions will be optimized for $\mathrm{D}^{+}$production, we expect that $10 \%$ to $30 \%$ of the extracted beam will consist of $\mathrm{D}_{2}^{+}$and $\mathrm{D}_{3}^{+}$.

\section{DESIGN CONSIDERATIONS: ACCELERATION- DECELERATION ELECTRODES}

It has been established in several ways ${ }^{B 10}$ that the required beam optics and beam density can be achieved if the long-term problems of voltage-holding, rigidity, thermal distortion, and mechanical tolerance can be solved. Therefore, the detailed optimization of the beam optics will be deferred until solutions are found to these other problems. 
The efficiency of convession of $\mathrm{D}^{+}$or $\mathrm{T}^{+}$to $\mathrm{D}^{0}$ or $T^{0}$ by electron capture from $D_{2}$ or $T_{2}$ gas is

$$
\begin{aligned}
F & =\frac{\sigma_{10}}{a_{10}+\sigma_{01}}\left(1-\exp \left(-\pi\left(\sigma_{10}+\sigma_{01}\right)\right)\right) \\
& =0.67 \text { for the assumed thickness } \pi .
\end{aligned}
$$

The cross sections for electron capture and for reionization are $\sigma_{10}=3.2 \times 10^{-16}$ and $\sigma_{01}=1.48$ $\times 10^{-16} \mathrm{~cm}^{2} /$ molecule for both $65-\mathrm{keV} D^{0}$ and 100-keV T0 ${ }^{088}$ Elastic scattering can be neglected at these energies; the only loss is by competition between the two charge-changing cross sections $\sigma_{10}$ and $a_{01}$. The exponent in this equation (equal to the average number of charge-changing collisions for each beam particle) is 3.8 , which implies that the neutralizer is essentially infinitely thick. The neutralizer thickness specified here is two or three times greater than the thickness required at lower energies because of the reduction in capture cross sections with energy. However, the efficiency $(F=67 \%)$ is still reasonably high. Electron capture is the most practical means of neutral production at this energy.

\section{Bcam-Line Pressure and Pumping Speed}

After the neutrals emerge from the neutralizer, they must be transported through the main beam line without there being excessive losses by reionizing collisions with the background gas. Therefore, the maximum allowable background pressure in the beam line may be specified by the requirement that not more than $5 \%$ of the neutral beam be lost by reionjzing collisions during the 3.5-m transit from the neutralizer through the main beam line to the reactor trapping chaniber; i.e.,

$$
\begin{aligned}
& \mathrm{n}_{\text {gas }} \sigma_{01} \mathrm{~L}<0.05 \\
& \mathrm{n}_{\text {gas }} \leqslant 10^{12} \text { molecules } / \mathrm{cm}^{3} \\
& \text { or } \mathrm{p}_{\text {gas }} \leqslant 3 \times 10^{-5} \text { Torr }
\end{aligned}
$$

It must also be ascertained whether the gas loads on the reactor plasma due to diffuse background gas and the direct streaming gas from the neutralizers are tolerable. As the computation carried out in Appendix A shows, thesc gas loads are tolerable and are smaller than the gas loads from the first wall. Beamline pumping may therefore be designed for the pressure requirement of $3 \times 10^{-5}$ Torr, for a total flow rate from the neutralizers of about $910 \mathrm{~A}$ of gas, and also for gas produced by dumping $107 \mathrm{~A}$ of monatomic charged beam. At a pressure of $3 \times 10^{-5} \mathrm{Torr}$, the required pumping speed would be impractically high $\left(6 \times 10^{6} \ell / \mathrm{s}\right.$. Therefore, to handle this large quantity of gas, most of it must be pumped at a higher pressure.

By installing an array of differential pressure ducts, as shown by Fig. 12 (page 13), a pressure ratio of 12/1 may be maintained between two pumping stages. A substantial economy can be achieved by pumping most of the gas at the higlier pressure of the first stage. Conductances will be adjusted so that the two pumping stages will operate with approximately equal pumping speeds in $\ell / \mathrm{s}$ in order to minimize the total requirement. Because of the unequal pressures, $92 \%$ of the gas will be pumped at the first stage. The results of a preliminary optimization are shown in Table B-2 and by the profile of gas pressures shown in Fig. B-2.

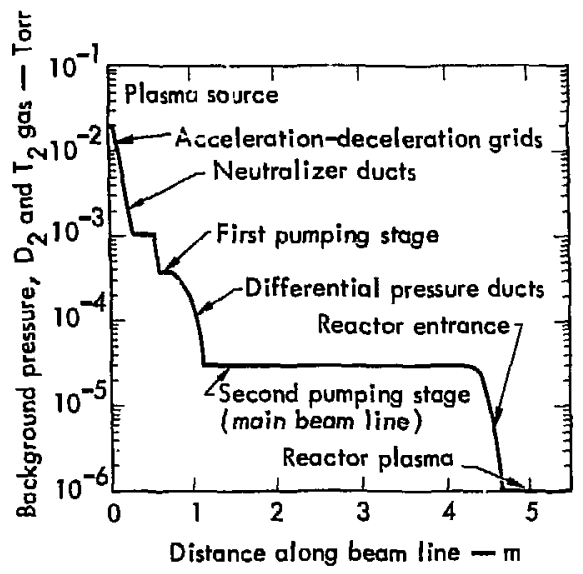

Fig. B-2. Gas-pressure profile along beam line. 


\section{Voltage and Spacing Requirements}

To avoid space-charge beam blowup, the applied electric fields should, on the average, be much larger than the space-charge electric fields in the accelerationdeceleration region where there are no neutralizing electrons; i.e.,

$$
\begin{gathered}
V_{\text {applied }} / d \gg j \mathrm{j} / \epsilon_{0^{v}} \\
\text { or } \mathrm{j}<<\epsilon_{0} \sqrt{2 \mathrm{q} / \mathrm{m}} \mathrm{v}^{3 / 2} / \mathrm{xd} \text {, }
\end{gathered}
$$

if $V_{\text {applied }}=1 / 2 \mathrm{~m} \mathrm{v}^{2} / \mathrm{q}$, and where $\mathrm{x}$ is the halfwidth of the beam. This space-charge limitation for beam blowup is more rigorous in most cases than the familiar Child's Law space-charge limitation for beam extraction:

$$
\mathrm{j} \leqslant \frac{4}{9} \epsilon_{0} \sqrt{2 \mathrm{q} / \mathrm{m}} \mathrm{v}^{3 / 2} / \mathrm{d}^{2} .
$$

Also, to prevent electron backstreaming, the deceleration voltage must be larger than the beam spacecharge potential; i.e.,

$$
V_{\text {decel }}>j x^{2} / 2 \epsilon_{0}^{v}
$$

To maximize the beam density, $j$, at a given velocity, $v$, we are led by these requirements to minimize the electrode gap, $d$, and the aperture half-width, $x$. Other limitations associated with the beam space charge also constrain the electrode dimensions to be small. The space-charge limitations are all related in general to voltage, beam density, and dimensions as shown by Eqs. (B1) and (B2). We now must ascertain the minimum practical values of the dimensions $d$ and $x$ which are consistent with FERF requirements for voltage-holding, rigidity, mechanical tolerance, and thermal distortion.

\section{Voltage-Holding Requirements}

The scaling law for breakdown across a low-pressure, high-voltage gap is usually reported as

$$
\mathrm{V}_{\text {breakdown }}=\mathrm{Cd}^{1 / 2} \text {, }
$$

where the empirical constant, $\mathrm{C}$, is typically found to have values between 46 and $60 \mathrm{kV} \cdot \mathrm{cm}^{-1 / 2}$ for ion source conditions and may be as high as $300 \mathrm{kV} \mathrm{cm}^{-1 / 2}$ for idealized high-vacuum conditions. The value of the exponent has been empirically debated, but with general agreement that the breakdown condition is nonlinear and that the exponent is less than unity. This scaling law depends very weakly upon the background gas pressure if the following conditions apply:

- The mean free path for ionizing collisions should be longer than the gap to avoid cascades of electrons. This effect can be seen from the Paschen curve, ${ }^{\text {Bl }} 1$ which shows the worst conditions for breakdown at $\mathrm{p}_{0} \mathrm{~d}=1$ Torr ${ }^{\mathrm{cm}}$. In the ion sources, we shall use $P_{0} d \leqslant 2 \times 10^{-3}$ Torr ${ }^{2} \mathrm{~cm}$, for which the Paschen curve does not apply.

- By eliminating all magnetic fields, we shall avoid pressure-dependent Penning discharges.

- Voltage gradients at surfaces of insulators must be substantially lower than voltage gradients across vacuum gaps. This requires stepped electrode mountings, which also serve as shields to protect the insulators from bombardment by particles and photors.

By combining the space-charge limitation with the voltage-holding limitation, we find that the maximum current density is attainable with the smallest possible value of $\mathbf{d}$ and with the correspondingly small value of $V_{\text {breakdown. Here we must recognize that } x \text { and } d}$ are not entirely independent, but must be related in some way ( $x=k d$, perhaps) to make the extraction geometry correct for beam optics.

$$
\begin{aligned}
& \mathrm{j} \propto \mathrm{V}^{3 / 2} / \mathrm{xd}=\left(C \mathrm{~d}^{1 / 2}\right)^{3 / 2} / \mathrm{kd}^{2} \\
& \propto \mathrm{d}^{-5 / 4} \propto \mathrm{V}_{\text {breakdown }}^{-5 / 2}
\end{aligned}
$$

This result indicates that at the first acceleration stage, where space-charge limitations are most severe, we

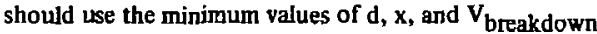
consistent with other requirements. The same limitations apply to other acceleration stages, but are less severe because of the higher velocity. Therefore, we may use higher voltages and larger gaps at the second and third acceleration stages.

\section{Electrode Design}

Figures 11 (page 12 ) and B-3 show a preliminary layout of a two-stage acceleration-deceleration electrode assembly designed for a total yoltage of $65 \mathrm{kV}$. 

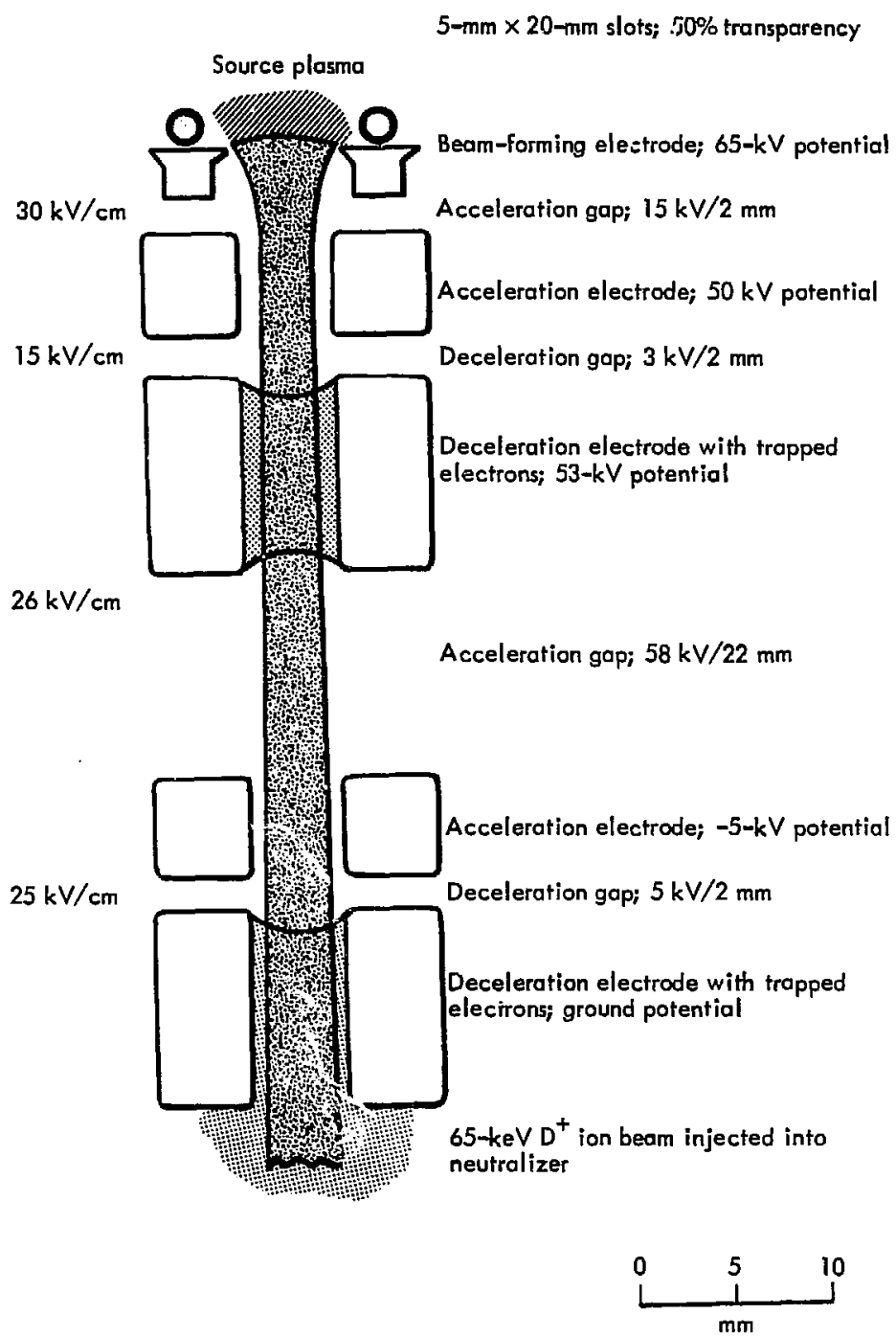

Fig. B-3. Two-stage acceleration-deceleration electrodes used for a total acceleration of $65 \mathrm{kV}$ (side view). 
The electrode dimensions shown by this layout are compatible with the relatively high voltage and the modest beam density required for FERF, but are two or three times larger than the electrodedimensions currently used for high-density pulsed beams. B10 Here we are guided largely by a series of tests in 1967-1968 which were directed toward development of a 5-A, 100-kV neutral injection system $B 12$ which essentially fulfilled the FERF requirements. These tests (limited at that time to an area of $3 \mathrm{~cm}^{2}$ and voltages up to $50 \mathrm{kV}$ ) indicated that the goals can be achieved with a larger source area and with a third acceleration-deceleration stage to attain a total of $100 \mathrm{kV}$. Recent improvements have occurred at several laboratories in respect to densities, optics, and areas of pulsed beams at voltages up to $40 \mathrm{kV}$. B10 The FERF voltage requirements (65 to $100 \mathrm{kV}$ ) represent an advanct urith respect to existing wide-area multiaperture as: ces, but have been greatly exceeded by single-ap : . . nurces such as the injectors for DCX-2. $\mathrm{B} 13$

The phenomena requiring consideration with respect to the higher voltage and continuous operation of FERF are electrode heating and sputtering, $x$-ray and gas evolution due to backstreaming electrons, mechanical rigidity and tolerances, and thermal distortion. It has been shown at several laboratories that the beam power density limited by these effects is at least $4 \mathrm{~kW} / \mathrm{cm}^{2}$ for large-area beams. This limitation is technological, not fundamental, and will probably be raised by improvements such as water-cooled grids.

Free electrons will be produced in large quantities throughout the assembly by ionization of the background gas, by bombardment of surfaces by ions, electrons, and photons, and by ionizing radiation. Backstreaming must be prevented by a deceleration stage at the end of the acceleration column and probably also (to suppress electron multiplication) by intermediate deceleration stages after each acceleration stage. Because electrostatically trapped electrons neutralize the beam space charge, the deceleration electrodes may be made relatively thick (perhaps $(0-\mathrm{mm})$ and therefore can be well cooled and will have a long sputtering lifetime. The thick deceleration electrodes can also be utilized to shield the thinner acceleration electrodes from ion and electron bombardment.
Electrodes can be cooled and mounted so that the thermal expansion is minimal and one dimensional. The stepped electrode mountings will accommodate the expansion and avoid thermal deformation. These design problems are simple with the reasonably large dimensions ( $-5-\mathrm{mm}$ ) shown by Fig. 11 and B-3 but are difficult with the thin grids required for highdensity pulsed beams.

Beam deflection due to electrode misalignment has been measured at several laboratories and has been found to vary inversely with aperture width ans electrode gaps. Therefore, we expect that misalignments of the order of $0.1 \mathrm{~mm}$ will be tolerable with respect to the \pm 14 degree FERF acceptance angle. This statement is extrapolated from beam deflections meạsured at LBL and Oak Ridge $B 14$ using sources operating at lower voltages and with smaller electrode dimensions; therefore, it will require verification under FERF conditions. The $0.1-\mathrm{mm}$ alignment tolerance is well within standard technology in respect to fabrication, assembly, rigidity, and thermal expansion.

\section{SOURCE RELIABILITY}

One of the critical requirements of the FERF injector system is the long-term reliability of source operation. Because of neutron activation and tritium contamination, it will be virtually impossible to work on the sources after they have been placed in operation. It will be necessary to assure reliable, maintenance-free performance of the source assemblies for at least 6 months $(4,000 \mathrm{~h})$. At the end of 6 months of FERF operation, it will be possible (if necessary) to replace the injectors with a completely new assembly. Probably, the used injectors must be discarded or stored until the level of activity permits maintenance work.

A large body of experience is available on the reliability of sources which have been continuously operated for long periods. This has been the subject of intensive development for electrostatic propulsion $B 15$ in which power densities and plasma densities were somewhat lower than, but with reliability exceeding, the FERF requirements. In this report, we shall refer to experience ac7erired in the course of many thousands of hours of con: MATS $^{B 2}$ icn s iurces. We have also compared experiences with many other laboratories. ${ }^{\mathrm{B} 17, \mathrm{~B} 18}$ The most 
important results were attained bectuse certain source components failed. We have learned, for example, that the lifetimes of oxide cathodes and graphite electrodes are limited by ion erosion and that Lucite insulators are subject to deformation under heat and stress. Such components must be replaced with more durable ones for the FERF injectors, although they may be satisfactory in other applications.

The op mating experience of the MATS sources is relevant to the lifetime projected for the FERF plasma sources. After a developmental period, both MATS I and MATS ${ }_{11}{ }^{\mathrm{B} 2}$ have been operated with no maintenance or repairs whatsoever for periods of one year, including over $1,000 \mathrm{~h}$ each of continuous Baseball operating time (up to $12 \mathrm{~h}$ per day), except for periodic replacement and recoating of oxide filaments and repairs to external water, gas, and electrical systems. At the end of these runs, the sources were disassembled and found to be in good condition except for one or two weak spots that are correctable by redesign.

The LLL Calutron ${ }^{B 16}$ bas also operated for several months with essentially no maintenance except for replacement of graphite electrodes. This experience provides some information on the lifetime of a tungsten filament (comparable in size and temperature to the FERF filaments) exposed to intense ion bombardment. Because of a $2.5 \times 7.5-\mathrm{mm}$ defining aperture limiting the 5- to 10-A arc current, the Calutron filament was subjected to ion bombardment with an arc current intensity of 25 to $50 \mathrm{~A} / \mathrm{cm}^{2}$. Under these conditions, the ion erosion was more severe than the tungsten evaporation; the lifetime of the $3-\mathrm{mm}$ filament was $300 \mathrm{~h}$. Therefore, we do not expect that ion erosion will determine the lifetime of the $8 \mathrm{~mm}$ FERF filaments which will operate with an arc current density of only $2 \mathrm{~A} / \mathrm{cm}^{2}$.

Long-term operating experience is also available at other laboratories, notably, for example, on the DCX sources at ORNL. 18 We prefer, however, to permit other laboratories to report their own experiences on source reliability, because the details of these experiences are largely unpublished.

A survey is being made by Battelle of the available data relevant to radiation damage to ion source insulators. Results of this survey will not be availab. in time for this report, but will necessarily be con- sidered in the choice of ins lator materials. If radiation effects are proven to bt severe in respect to ion scurce insulators, it may be nicessary to shield the ion sources from neutrons ant other radiation by using a magnetic guide field ${ }^{B 2}$, wher than converging neutral beams.

By drawing upon such experiences and using care in design, selection of materials, and source assembly, we expect that the FERF reliability requirement is attainable. A developmental period under realistic operating conditions will be required.

\section{INJECTOR POWER SUPPLY REQUIREMENTS}

The requirements for injector power supplies are determined by the requirements of the injected beam (see Table B-1) and by the injector design. Table B-3 shows the injector power supply requirements and the total connected load determined by these requirements. However, for the reasons listed below, the average power required by the injectors will be only about $75 \%$ of the total connected load:

- On the average, the nominal requirement for a 500-A injected beam will be fulfuled by the operation of not more than 3.5 beam lines.

- The filament power requirement may be reduced by developing low-temperature cathodes with the required lifetime. The filament power requirement will also be reduced by heat from adjacent filaments, from the arc plasma, and from heat shields.

- The filament power requirement may be reduced by developing low-temperature cathodes with the required lifetime. The filament power requirement will also be reduced by heat from adjacent filaments, from the arc plasma, and from heat shields.

- The deceleration current actually supplied will be determined by the ion beam intercepted by the deceleration electrodes. This beam will be less than $2 \%$ of the total beam. However, to maintain constant voltages is spite of source fluctuations, the deceleration power supplies must be current-rated for $20 \%$ of the total beam current. This constirutes a small fraction of the total, connected power load. 
Table B-3. Injectar power supply requirements determined by injected beam requirements shown by Table B-l.

\begin{tabular}{|c|c|c|c|}
\hline & Deuterium & Tritium & Total \\
\hline Number of modules in 4 heam lines & 72 & 40 & 112 \\
\hline \multicolumn{4}{|l|}{ Acceleration power supply requirements: } \\
\hline Total voltage; sum of 2 or 3 stages $(\mathrm{kV})$ & 65 & 100 & \\
\hline Current $/$ module at $4 \mathrm{~kW} / \mathrm{cm}^{2}(\hat{A})$ & 12.6 & 8.4 & \\
\hline Total acceleration current $(A)$ & 907 & 336 & 1,243 \\
\hline Total acceleration power (MW) & 59 & 33.6 & 92.6 \\
\hline \multicolumn{4}{|l|}{ Deceleration power supplies } \\
\hline $\begin{array}{l}\text { Total voltage, possibly required for sum of } 2 \text { or } 3 \\
\text { deceleration stages (kV) }\end{array}$ & 15 & 15 & \\
\hline Deceleration current; $20 \%$ of acceleration current (A) & 194 & 67 & 261 \\
\hline Deceleration power (MW) & 2.91 & 1.01 & 3.92 \\
\hline \multicolumn{4}{|l|}{ Arc power: } \\
\hline Asc current/module (kA) & 2.1 & 2.1 & \\
\hline Arc voltage $(\mathrm{V})$ & 50 & 50 & \\
\hline Arc power/module (kW) & 105 & 105 & \\
\hline Arc power, total (MW) & 7.56 & 4.2 & 11.76 \\
\hline \multicolumn{4}{|l|}{ Filament power: } \\
\hline Filament current, each conductor $(\mathrm{kA})$ & 1.25 & 1.25 & \\
\hline Filament voltage; $\mathrm{rms}, 400 \mathrm{~Hz}(\mathrm{~V})$ & 8 & 8 & \\
\hline Filament power, 12 conductors/module (kW) & 120 & 120 & \\
\hline Total filament power (MW) & 8.64 & 4.8 & 13.44 \\
\hline \multicolumn{4}{|l|}{ Total connected load for 112 modules (MW): } \\
\hline Acceleration power & 59.00 & 33.60 & 92.60 \\
\hline Deceleration power & 2.91 & 1.01 & 3.92 \\
\hline Arc power & 7.56 & 4.20 & 11.76 \\
\hline Filament power & 8.64 & 4.80 & 13.44 \\
\hline TOTAL & 78.11 & 43.61 & 121.72 \\
\hline
\end{tabular}


Systems schematics for a 65-kV, 8.3-A neutral deuterium source and for a $100-\mathrm{keV}, 5.6-\mathrm{A}$ neutral tritium source are shown in Figs. B-4 and B-5, respectively. These sources are basically scaled-up versions of our present sources. The cathode area is increased and the emission density is reduced so that the life expectancy would be about 6 months $(4,000 \mathrm{~h})$. To maximize the filament life by compensating for the effects of cathode evaporation, the filament voltage is regulated and programmed over the 6-month period by the control computer.
For this study, it is assumed that a pure tungsten filament would be used. Based on this assumption. the cathode power for each source is about $120 \mathrm{kVA}$. Hecause the filament voltage must be below the first ionization potential (about $15 \mathrm{~V}$ ), the filament current is very high and the filament transformer must be located near each filament. Because the large concentration of sources makes it difficult to accommodate large 60-Hz transfurmers, we chose to go to smaller, 400-Hz urits.

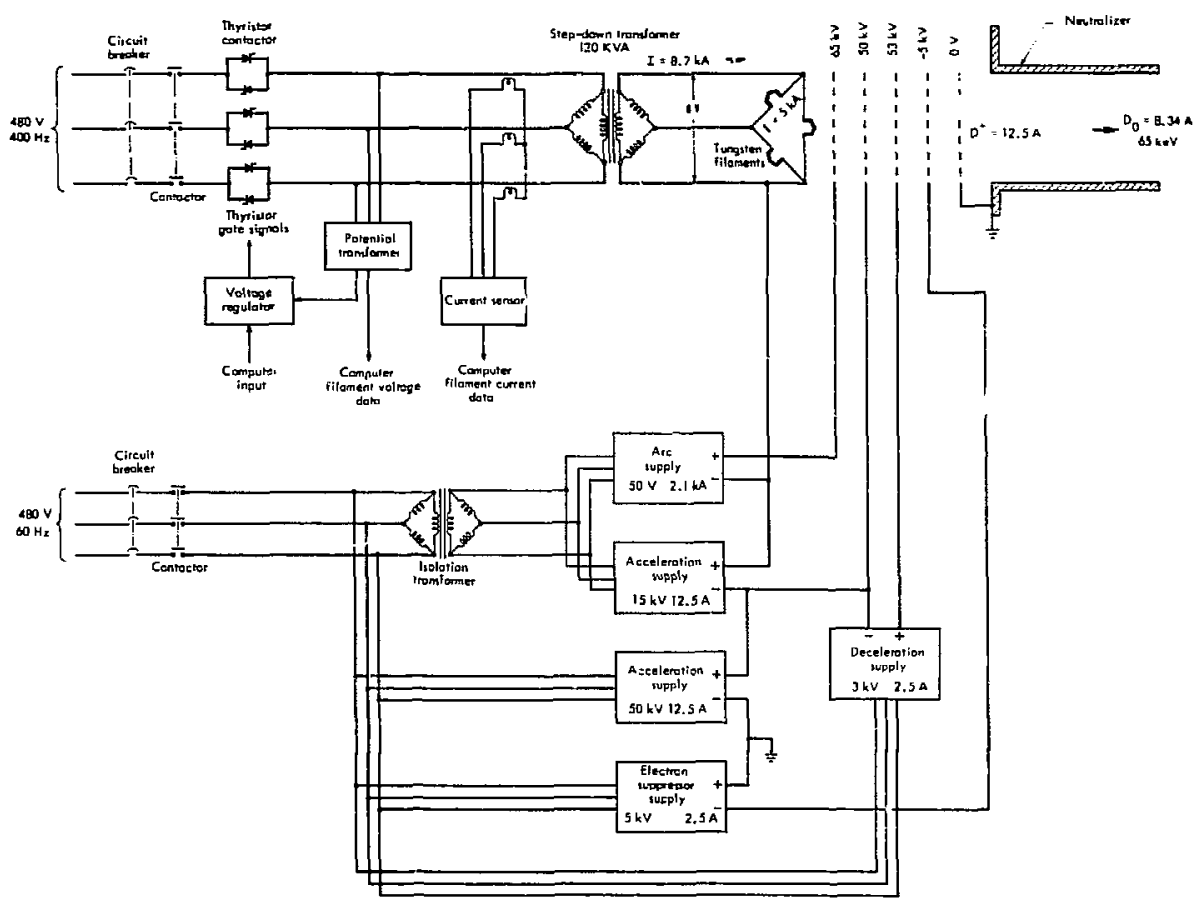

Fig. B-4. Ion-source power-supply interconnections ( $65 \mathrm{kV}, 8.3-\mathrm{A}$ neutral deuterium source). 



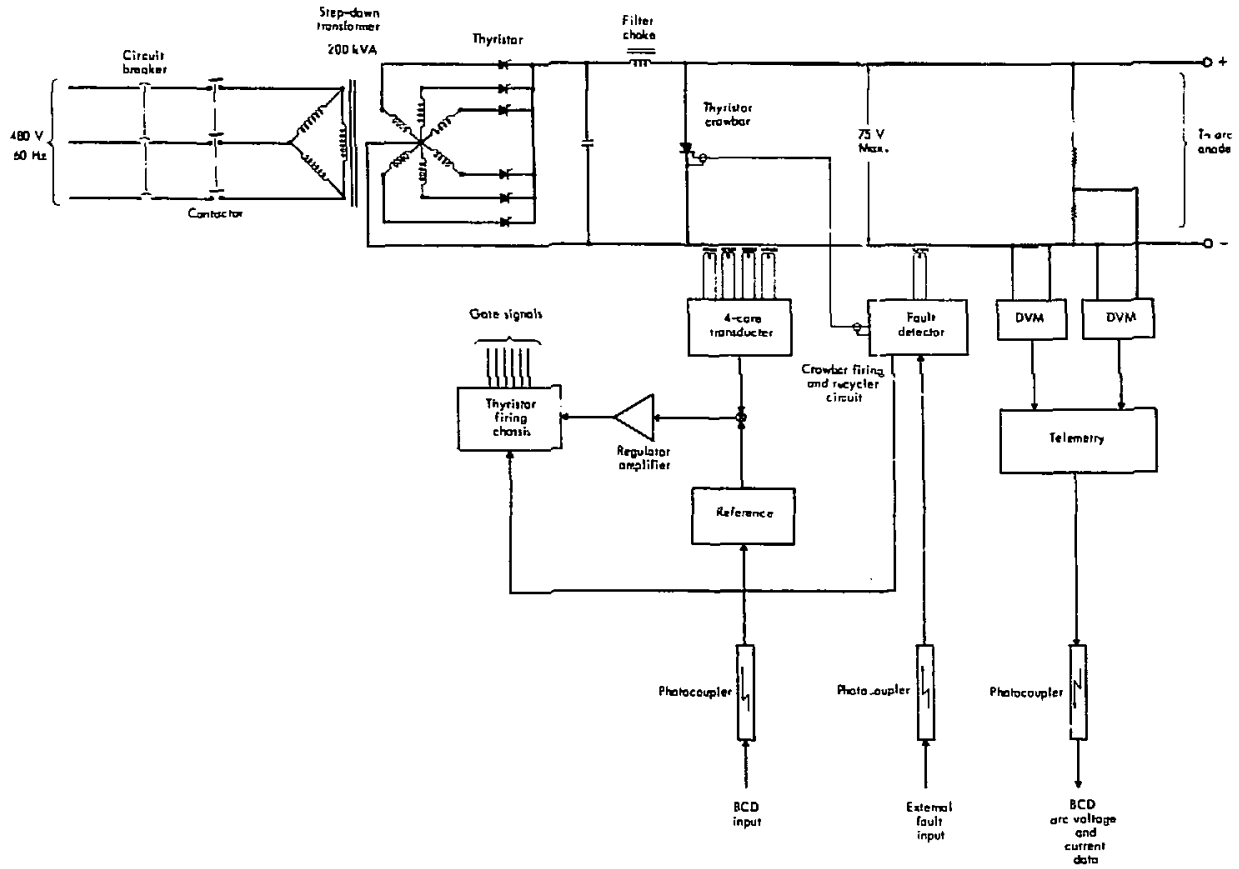

Fig. B.6. Arc power-supply circuit schematic.

\section{Acceleration-Electrode Power Supplies}

The power supplies for the acceleration e!ectrode are voltage-regulated, thyristor-controlled, power supplies with a filter to remove the ripple. A typical supply schematic is shown in Fig. B-7. Eacil supply contains an ignition crowbar and a voltage regulator. The fault detector senses overcurrent, fires the ignition and sends a turnoff signal to the firing chassis of the ignition. The fault detector contsins a recycler to restablish voltage once the products of the fault have been cleared. The interlock chain and voltage monitoring are handled in the same way as in the arc power supply.

The power supplies for the deceleration electrode are similar except for being very much smaller.

\section{NEUTRAL BEAM CONTROL EQUIPMENT}

Each neutral beam source is a straightforward piece. of electronic equipment so far as electrical excitation and control are concerned. That is, each is simply a scaled-up version of the neutral beam sources which we routinely operate. However, the increase in size, voltage, and number of sources coupled with the hostile environment in which they operate makes the problem more formidable. Under these circumstances, it is best to make each source operate automatically and to restrict information transmitted between the control room and the source to the minimum essential for proper control. Even with minimum information transmission, the quantity is still beyond the capability of a human operator with only a manual con- 


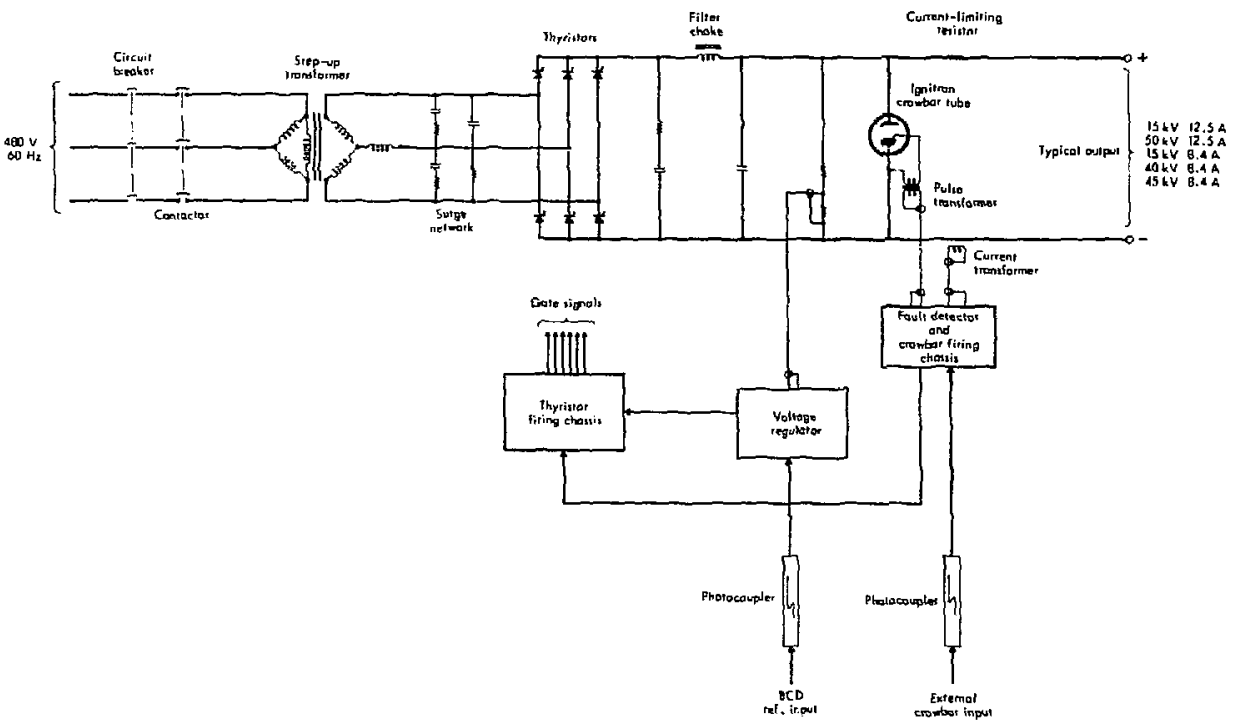

Fig. B-7. Acceleration power-supply circuit schematic.

trol system. However, with a controi computer to accumulate, organize, and present the data to the operator, a thorough understanding of the simultaneous operation of these sources can easily be achieved. Also, the computer's ability to transmit programmed commands according to the operator's judgment enables a single operator to control the large source array with a high degree of efficiency.

\section{LONG-LIFE FILAMENT DESIGN}

The plasma source for each injector modute will include three extraction areas (see Fig. 12 page 13 ), each being a $2 \times 35 \mathrm{~cm}$ array through which the average extracted beam density is $60 \mathrm{~mA} / \mathrm{cm}^{2}$ for $D^{+}$ or $40 \mathrm{~mA} / \mathrm{cm}^{2}$ for $\mathrm{T}^{+}$. With the allowance for the blank spaces between the extraction areas, plasma must be produced for $21 \mathrm{~A}$ of ions. This requires a 2100-A arc current per module which must be emitted thermionically to prevent plasma fuctuations at the cathodes.
To avoid frequent replacement and repair of components activated by neutrons, a lifetime of about 6 months is required for all source components. The most predictable cathode materials for the long lifetime are refractory metals (such as pure tungsten) for which the lifetime is determined by evaporation and not by ion bombardment. Although thin-film cathodes such as oxides and thoriated tungsten are preferable because of their lower work functions and Iower power requirements, they are subject to sputtering and erosion and therefore cannot be relied upon until they have been tested and proven suitable.

Tungsten cathodes can be heated to the required emission temperature either ohmically (at a voltage Iess than the arc voltage) or indirectly (with higher voltage and lower current). Ohmic heating, although oppearing simpler requires a large filament transformer and a sufficiently large filament cross section to prevent hot spots from developing because of nonuniform evaporation. 
According to standard practice, we will require that the ohmically heated filaments not reduce their diameter by evaporation by more than $6 \%$ during their 6-month lifetime. Using a $3 \times 10^{-8} \mathrm{~g} / \mathrm{cm}^{2}$.s evaporation rate, we find this requires a relatively low temperature $(2700 \mathrm{~K})$ and a heavy tungsten filament $(0.8-\mathrm{cm}$ diameter). The temperature is low only in comparison with the pulsed filaments $(3200 \mathrm{~K})$ at LBL. Most tungsten filaments operate at even lower temperatures.

The dependence of thermal radiation, thermionic emission, and evaporation rate upon the temperature of a tungsten filament must be considered. The thermal radiation rises with the fourth power of the temperature; the thermionic emission and evaporation rise exponentially, much more steeply. Therefore, when we reduce the temperature from $3200 \mathrm{~K}$ to $2700 \mathrm{~K}$, we reduce the evaporation per unit area by a factor of 250 and the thermionic emission by a factor of 29 , but we reduce the thermal radiation only by a factor of 2. Consequently, to maintain the same emission we must provide 29 times nore area, and the total heat load goes up by 14.5. This price is paid to improve the lifetime of the filament by a factor of 4000.

We can compute $\mathrm{R}^{\mathrm{B} 9}$ the specifications for the FERF filament and compare them to those for the LBL 10-A pulsed source. The tabulation (Table B-4) indicates that an array of $0.8-\mathrm{cm}$-diameter filaments would operate satisfactorily at $2700 \mathrm{~K}$, but would constitute a large heat load. The $120-\mathrm{kW}$ requirement for filament power will be reduced by reflected heat from heat shields and from adjacent filaments.

Table B-4. Specifications for ohmically heated tungsten filaments.

\begin{tabular}{|c|c|c|}
\hline & FERF module & $\begin{array}{l}\text { LBL 10-A pulsed } \\
\text { source }\end{array}$ \\
\hline Filament temperature $(K)$ & 2700 & 3200 \\
\hline Total emission requirement (A) & 2100 & 1940 \\
\hline Thermionic emission density $\left(\mathrm{A} / \mathrm{cm}^{2}\right)$ & 2 & 57 \\
\hline Emission area required $\left(\mathrm{cm}^{2}\right)$ & 1050 & 34 \\
\hline Design lifetime (continuous) & 4,000 & 1.2 (hours) \\
\hline Filament diameter required for lifetime $(\mathrm{cm})$ & 0.8 & 0.05 \\
\hline Filament current required for temperature $\mathrm{a}^{\mathrm{a}}$ (A) & 1250 & 26 \\
\hline Total length of conductor $=$ area $/ \pi \mathrm{D}(\mathrm{cm})$ & 420 & 216 \\
\hline Filament voltage desired $(V)$ & 8 (rms) & 14 \\
\hline Voltage/length ${ }^{\mathbf{a}}(\mathrm{V} / \mathrm{cm})$ & 0.225 & 1.3 \\
\hline Length of each conductor $(\mathrm{cm})$ & 35 & 10.8 \\
\hline Number of conductors in parallel (connected for 3-phase a.c.) & 12 & 20 \\
\hline Shape of filament array & rectangular & circular cylinder \\
\hline Dimensions (cm) & $16 \times 41 \times 5.4$ & $13 \times 5.4$ \\
\hline Perimeter (cm) & 114 & 41 \\
\hline Area of filament array $\left(\mathrm{cm}^{2}\right)$ & 616 & 221 \\
\hline \multicolumn{3}{|l|}{ Spacing between conductors $=$ area/total length of conductors } \\
\hline Center-to-center (cm) & 1.5 & 1.0 \\
\hline Clear spacing (cm) & 0.7 & 0.95 \\
\hline \multicolumn{3}{|l|}{ Magnetic field at conductor surface due to filament current; } \\
\hline $\mathrm{B}=\mu_{\mathrm{o}} \mathrm{I} / 2 \pi \mathrm{r}(\mathrm{T})$ & 62.5 & 30.8 \\
\hline Total filament current; each feed-through; delta-connected (kA) & 8.7 & .500 \\
\hline $\begin{array}{l}\text { Total filament power (neglecting effects of reflected } \\
\text { heat and heat from adjacent filaments) (kW) }\end{array}$ & 120 & 7 \\
\hline
\end{tabular}

${ }^{a}$ Reference B19. 
The magnetic forces and the temperature fuctuations due to the a.c. heating current have been computed and found negligible except for a time-averaged, frequency-independent magnetic force which may be a maximum of 2.2 times the force of gravity upon a conductor. Although the $400-\mathrm{Hz}$ heating frequency is preferred in order to economize on the size of the transformer coie, it is not required for thermal or mechanical reasons. The filament temperature, which will be affected by heat reflected fro, h heat shiclds, from adjacent filaments, and from the arc plasma, will be sensed by the filament resistance and regulated by the transformer.

\section{REFERENCES}

B1. H. C. Cole, D. P. Hammon, E. M. Jones, A. C. Riviere, and J. Sheffield, "Electrode Heat Dissipation Limits on Multi-Aperture Ion Source Performance", in Proc. of the Second Internationai Confetence on Ion Sources, 1972 (Vienna, Austria, (1972), p. 271.

B2. G. W. Hamilton and J. E. Osher, "A Design Study of a 14,000-Ampere Injection System for a Fusion Reactor" Rept. UCRL-74096 LLL, (1972).

B3. A. C. Riviere, Neutral Injection Heating of Toroidal Reactors, Appendix 3, Culham Laboratory, United Kingdom, Rept. C.MMR-112 (1971).

B4. K. H. Ehlers, W. R. Baker, K. H. Berneer, W. S. Cooper, W. B. Kunkel, R. V. Pyle, and J. W. Stearns, Design and Operation of an Intense Neutral Beam Source, Lawrence Berkeley Laboratory, Rept. LBL-1589 (1973).

B5. W. L. Stirling, R. C. Davis, T. C. Jernigan, O. B. Morgan, T. J. Orzechowski, G. Schilling, and L. D. Stewart, "Intense Ion Beam Production Below $40 \mathrm{keV",} \mathrm{in} \mathrm{Proc.} \mathrm{of} \mathrm{the} \mathrm{Second} \mathrm{Interna-}$ tional Conference on Ion Sources (Vienna, Austria, 1972),p. 278.

B6. W. C. Cooper, K. B. Berkner, and R. V. Pyle, "Multiple-Aperture Extractor Design for Producing Intense Ion and NeuiraI Beams", submitted to Applied Physics Letters (197I).
B7. J. E. Osher and G. W. Hamilton, "An Intense I-kV Ion Source for Controlled Fusion Research", in Proc. of the Symposium on Ion Sources and Formation of Ion Beams (Brookhaven, N. Y., 1971).

B8. H. Tawara and A. Russek, "Charge Changing Processes in Hydrogen Beams", Rev. Mod. Phys. 45, 178 (1973)

B9. K. W. Ehlets, Bull. Am. Phys. Soc. 18, 1296 (1973).

B10. Proc. of the Second International Conference on Ion Sources (Vienna, Austria, 1972), papers B-6, D-3, D-5.

B11. M. J. Druyvesteyn and F. M. Penning, Rev. Mod Phys. 12, 87 (1940)

B 12. Research in High-Temperature Plasma, AerojetGeneral, Rept. AFAPL-TR-68 (I968); also FiveAmpere Multimomentum Injection System for Controlled Fusion Reseirch, Aerojet-General, Rept. SRRO-68-4 (1968).

B13. O. B. Morgan, G. G. Kelley, R. C. Davis, Rev. Sci. Instr. 38, 467 (1967).

BI4. R. F. Pyle, Lawrence Berkeley Laboratory, and L. D. Stewart, ORNL, private communications (1974).

B1 S. D. B. Langmuir, E. Stuhlinger, and J. M. Sellen, Jr., Eds., Electrostatic Propulsion (Academic Press, New York, 1961).

B16. F. J. Gordon and C. C. Damm, "High Intensity Source of 20-keV Hydrogen Atoms", Rev. Sci. Inst. 34, 963 (1963).

B17. G. W. Hamilton, "Ion Source Technology", in Proc. of the Symposium on Ion Sources and Formation of Ion Beams (Brookhaven National Laboratory, (971).

B18. O. B. Morgan, G. G. Kelley, and R. C. Davis, Rev. Sci. Instr. 38, 467 (1967).

B19. W. H. Kohl, Handbook of Materials and Techniques for Vacuum Devices (Reinhold, N. Y., I967). 


\section{Appendix C - Wall Bombardment by Neutral Atoms}

\section{INTRODUCTION}

A fraction of the injected neutral beam is ionized and trapped in the FERF plasma. The remainder of the neutral particles* either penetrate through the plasma or charge-exchange with plasma ions. The penetrating neutrals impact the first wall. The neutral atoms formed in the charge-exchange collisions, if they do not undergo reionization, also impact the first wall. The first wall absorbs the energy of the impacting neutrals, may be eroded by sputtering, and re-emits the particles as cold gas. Knowledge of injection-beam/plasma interaction is vital to FERF design.

- The trapped fraction of the injected beam must be known in order to size the injector system.

- The magnitude and distribution of tine wall bombardment is important to the design of the first wall.

- The magnitude of the cold gas re-emission is important to the prediction of plasma behavior. The magnitude of the wall bol ihardment is very energysensitive, and the distribution is very nonunifom because the cross section for charge-exchange decreases steeply with increasing relative velocity between plasma ion and injected neutral.

The FERF plasma has a nonuniform ion density, non-Maxwellian ion energy distributicn, nonisotropic ion velocity distribution, and a complex shape. These characteristics plus the multitude of injector beam lines make a complete analysis of injection-beam/ plasma interaction very difficult. However, an approximate analysis has been carried out which retains two very important aspects of the problem, namely, the energy distribution of the plasma ions and the relative velocity dependence of the chargeexchange cross section. Simplifying assumptions in the analysis include a uniform ion density, an isotropic angular distribution of plasma ion velocities, and a spherical plasma geometry. Tha analysis yields the distributions of the neutral particle flux and power density at the surface of the plasma as functions of

"Henceforth referred to as "neutral(s)." the angle relative to the beam axis, the injection energy, the plasma energy distribution, and the beam attenuation thickness of the plasma. General results are discussed and compared to those of a previous, more simple analysis. ${ }^{\mathrm{Cl}}$ Then the results are used to predict the peak and average neutral particle flux and thermal load on the first wall of FERF.

\section{ANALYTIC MODEL}

The model used for the approximate analysis is shown in Fig. C-1. A monenergetic, single-species neutral of energy $E_{0}$ (velocity $v_{0}$ ) and particle current $\mathrm{I}_{0}$ is injected diametrically across a spherical plasma of radius $r_{p}$. The beam diameter is infinitesimal. The plasma has a uniform density, $n$, of a single ion species, an arbitrary energy distribution $f\left(E_{p}\right)$, and an isotropic velocity distribution.

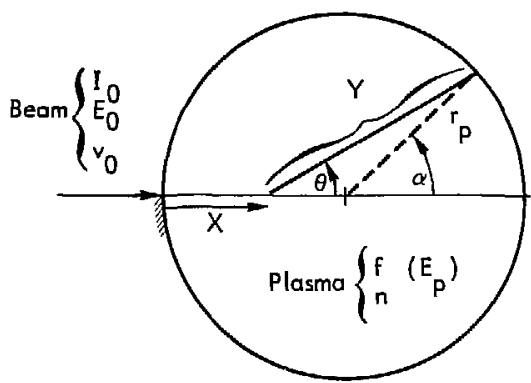

Fig. C-1. Analy tic model.

At a position $x$ along the beam path, the neutral beam current has attenuated due to ionization and charge-exchange collisions with plasma ions. The beam neutrals which are ionized in either type of collision are assumed to be trapped on magnetic field lines passing through the plasma. At $x=2 r_{p}$, the surviving neutral beam particles are called penetrating neutrals. A charge-exchange collision which occurs at position $x$ between a beam neutral and a plasma ion with energy $E_{p}$ and velocity vector inclination to the beam path $\theta$ results in a neutralized plasma ion at the same energy $E_{p}$ and the same angle $\theta$, where $0 \leqslant \theta \leqslant \pi$. 
Charge-exchange collisions with forwardiy directed plasma ions predominate because the charge-exchange cross section is large for particle pairs with small relative velocities. The newly formed charge-exchange neutral particle* of energy $E_{p}$ and inclination $\theta$ must travel the distance $y$ to reach the surface of the plasma. It may undergo an ionization or charge-exchange collision which results in trapping and thus not reach the surface. Of course, if it charge-exchanges, then another neutral is born. In the present model, this secondgeneration charge exchange is neglected, and the attenuation of the charge-exchange-neutral current along the path $y$ is assumed to be by ionization only. The total current density of neutrals reaching the surface of the plasma is the sum of the penetrating neutrals and the charge-exchange neutrals which do not reionize, and is geometrically dependent only on the angle $\alpha$ shown in Fig. C-1.

\section{MATHEMATICAL DESCRIPTION}

At position $x$, the rate of production of chargeexchange neutrals of energy $E_{p}$ emitted at angle $\theta$ which reach the surface of the plasma is

$$
\begin{aligned}
& \frac{\mathrm{d}^{3} \mathrm{I}_{\mathrm{cx}}}{\mathrm{dE} \mathrm{E}_{\mathrm{p}} \mathrm{d} \theta \mathrm{dx}}=\left[\frac{\mathrm{J}_{0}}{\mathrm{v}_{0}} \exp \left(-\frac{\mathrm{x}}{\lambda_{\mathrm{T}}}\right)\right]\left[\frac{\mathrm{l}}{2} \sin \theta \operatorname{rf}\left(\mathrm{E}_{\mathrm{p}}\right)\right. \\
& \left.\mathrm{x} \sigma_{\mathrm{cx}} \mathrm{v}_{\mathrm{c}}\right]\left[\exp \left(-\frac{\mathrm{y}}{\lambda_{\mathrm{i}}}\right)\right],
\end{aligned}
$$

where $\lambda_{\mathrm{T}}$ is the total mean free path for the injected neutral (taking into account both ionization and charge exchange), $\lambda_{i}$ is the ionization mean free path for the charge-exchange neutral, $\sigma_{\mathrm{cx}}$ is the microscopic cross section for charge exchange, and $v_{c}$ is the collision velocity between the injected neutral and the plasma ion; i.e.,

$$
v_{c}=\left(v_{0}^{2}-2 v_{0} v_{p} \cos \theta+v_{p}^{2}\right)^{1 / 2}
$$

where $v_{p}$ is the plasma ion velocity.

"Henceforth referred to as "charge-exchange neutral(s)."
The first square-bracketed term of Eq. (Cl) gives the number of injected neutrals in a length $d x$, taking into account the attenuation due to both ionization and charge exchange. The second square-bracketed term of Eq. (CI) gives the charge-exchange reaction rate per injected neutral per unit $\mathrm{d} \theta$ and $\mathrm{dE}_{\mathrm{p}}$. Note that division of this term by $\mathbf{n}$ followed by double integration over all $\theta$ and $E_{p}$ yields the charge-exchange reaction rate coefficient as given by Riviere: ${ }^{C 2}$

$$
\begin{aligned}
& \left\langle\sigma v_{c}\right\rangle_{c x}=\int_{E_{p, M I N}}^{E_{p, M A X}} f\left(E_{p}\right) \\
& {\left[\int_{0}^{\pi} \sigma_{c x} v_{c} \frac{1}{2} \sin \theta d \theta\right] d E_{p} .}
\end{aligned}
$$

The ionization reaction rate coefficient, $\left\langle\sigma v_{c}\right\rangle_{i}$, is evaluated in the same way as $\left\langle\sigma v_{c}\right\rangle_{c x}$. The total reaction-rate coefficient is defined as

$$
\left\langle\sigma v_{c}\right\rangle_{T}=\left\langle\sigma v_{c}\right\rangle_{c x}+\left\langle\sigma v_{c}\right\rangle_{i}
$$

The third square-bracketed term of Eq. (CI) is the at tenuation of the charge-exchange neutrals due to reionization as they travel the distance $y$ to reach the surface of the plasma. From Eq. (Cl), y is given by

$$
\mathbf{y}=\mathbf{r}_{\mathbf{p}} \frac{\sin \alpha}{\sin \theta},
$$

and by the law of sines, $\alpha$ and $\theta$ can be shown to be related by

$$
\tan \theta=\frac{\sin \alpha}{1-\mathrm{x} / \mathrm{r}_{\mathrm{p}}+\cos \alpha} .
$$

We define a plasma-attenuation parameter, $\gamma$, as

$$
\gamma=\frac{2 r_{p}}{\lambda_{T}}
$$

Thus, by definition, the fraction of injected neutrals which penetrate entirely through the plasma is $\mathrm{e}^{-\gamma}$. 
Recall that a mean free path may be defined in general by

$$
\lambda \equiv \frac{v}{\bar{n}\left(\sigma v_{c}\right)}
$$

Using Eqs. (C6) and (C7), we rewrite Eq. (C1) as

$$
\begin{gathered}
\frac{d^{3} I_{c x}}{d E_{p} d \theta d\left(x / r_{p}\right\rangle}=\frac{I_{0} \gamma}{4} \frac{\sigma_{c x} v_{c}}{\left\langle\sigma v_{c}\right\rangle_{T}}-f\left(E_{p}\right) \sin \theta \exp \\
\quad \times\left(-\frac{x}{2 r_{p}}-\gamma\right) \exp \left(-\frac{y}{2 r_{p}} \frac{v_{0}\left\langle v_{c}^{*}\right\rangle_{i}}{v_{p}\left\langle\sigma v_{c}\right\rangle_{T}} \gamma\right)
\end{gathered}
$$

Note that $v_{c}^{*}$ is the collision velocity between the charge-exchange neutral and the plasma ion and is not the same as $v_{c}$.

Along with Eq. $(C 8)$, we require analytic relations for $f\left(E_{p}\right), \sigma_{c x},\left\langle\sigma v_{c}\right\rangle_{T}$, and $\left\langle\sigma v_{c}\right\rangle_{i} /\left\langle\sigma v_{c}\right\rangle_{T}$. For the plasma ion energy distribution $f\left(E_{p}\right)$, we use the analytic expressions given by Riviere $C 2$ for a mirrorconfined plasma with mirror ratio $R=3$ :

$$
\begin{aligned}
& f\left(\frac{E_{p}}{E_{0}}\right)=0 \text { for } \frac{E_{p}}{E_{0}}<0.18 \\
& f\left(\frac{E_{p}}{E_{0}}\right)=-1.316\left(\frac{E_{p}}{E_{0}}\right)^{2}+2.831\left(\frac{E_{p}}{E_{0}}\right) \\
& -0.4674 \text { for } 0.18 \leqslant \frac{E_{p}}{E_{0}} \leqslant 1 \\
& f\left(\frac{E_{p}}{E_{0}}\right)=1.684-1.0526\left[-3.69+5\left(\frac{E_{p}}{E_{0}}\right)\right. \\
& \left.-\left(\frac{E_{p}}{E_{0}}\right)^{2}\right]_{\text {for } 1 / 2}^{1 / 2} \leqslant \frac{E_{p}}{E_{0}} \leqslant 2.5 .
\end{aligned}
$$

The energy distribution is nomalized such that

$$
\int_{0}^{2.5} f\left(\frac{E_{p}}{E_{0}}\right) d\left(\frac{E_{p}}{E_{0}}\right)=1
$$

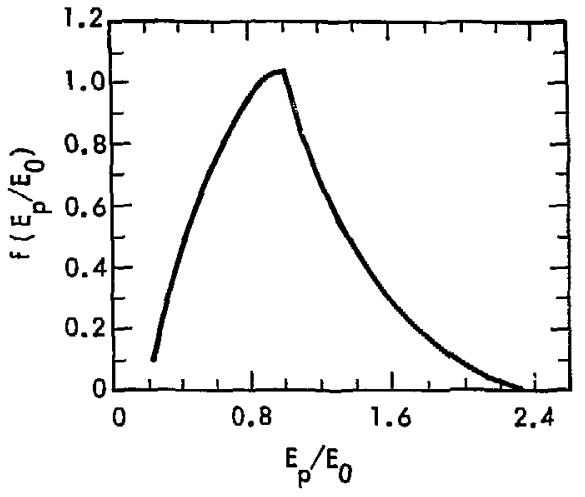

Fig- C-2. Energy distribution of plasma ions.

Equation (C9) is plotted in Fig. C-2. Note that the energy distribution peaks at the injection energy $E_{0}$.

For the charge-exchange cross section $\sigma_{\mathrm{cx}}$, we use the expression given by Riviere ${ }^{C 2}$ for protons in atomic hydrogen:

$\sigma_{c x}=\frac{0.6937 \times 10^{-14}\left(1-0.155 \log _{10} E_{c}\right)^{2}}{1+0.1112 \times 10^{-14} E_{c}^{3.3}} \mathrm{~cm}^{2}$,

where the collision energy $E_{\mathcal{C}}$ is expressed in electron volts. For $D$ injected into $D$ or $T$ injected into $T$, we use Eq. (C10) after calculating $E_{c}$ from

$$
E_{c}=\frac{1}{2} \frac{\left.m_{D(o r} T\right) v_{c}^{2}}{e}
$$

Equation (C10) agrees very well with data plotted by Riviere for $100 \leqslant \mathrm{E}_{\mathrm{c}} \leqslant 10^{5} \mathrm{eV}$ and yields a monotonically decreasing charge-exchange cross section with increasing $E_{c}$. For $E_{c}<100 \mathrm{eV}$, Riviere shows no data, and for such cases we calculate $\sigma_{\mathrm{cx}}$ assuming $\mathrm{E}_{\mathrm{c}}=100 \mathrm{eV}$.

For $\operatorname{lov}_{\mathrm{c}} \mathcal{J}_{\mathrm{T}}$, we use the following approximation to a curve calculated by Riviere $C 2$ for $D$ injected into $D$ :

$$
\left\langle\sigma \mathrm{v}_{\mathrm{c}}\right\rangle_{\mathrm{T}}=5.90 \times 10^{-7} \mathrm{E}_{0}^{-0.42} \mathrm{~cm}^{3 / \mathrm{s}}
$$

where the injection energy $E_{0}$ is expressed in $\mathrm{keV}$. For $T$ injected into $T, E_{0}$ should be replaced by $2 E_{0} / 3$. 
For $\left\langle\sigma v_{c}^{*}\right\rangle_{i} /\left\langle\sigma v_{c}\right\rangle_{T}$, we first make the simplifying assumption that $\left\langle\sigma v_{c}^{*}\right\rangle_{i} \cong\left\langle\sigma v_{c}\right\rangle_{j}$, i.e., that the ionization reaction rate coefficient is the same for charge-exchange neutrals and injected neutrals. Then, we use the following approximation to curves calculated by Riviere ${ }^{\mathrm{C} 2}$ for $D$ injected into $D$ :

$$
\frac{\left\langle\sigma v_{c}\right\rangle_{i}}{\left\langle\sigma v_{c}\right\rangle_{T}}=0.184 \mathrm{E}_{0}^{0.29}
$$

where the injection energy $E_{0}$ is expressed in keV.

For $T$ injected into $T, E_{0}$ should be replaced by $2 E_{0} / 3$. Equations $(\mathrm{Cl} 2)$ and $(\mathrm{Cl} 3)$ match Riviere's results within $10 \%$ in the range $40 \leqslant \mathrm{E}_{0} \leqslant 400 \mathrm{keV}$.

The substitution of Eqs. (C9), (C10), ( $\mathrm{Cl} 2$ ), and (C13) into Eq. (C8) completes the mathematical description of the problem.

\section{RESULTS OF ANALYSIS}

The triple integration of Eq. (C8) over all $E_{p}, \theta$, and $x$ yields the ratio $I_{c x} / l_{0}$ as a function of injection energy $E_{0}$ and attenuation parameter $\gamma$. The integral is written as

$$
\begin{aligned}
& \frac{I_{c x}}{I_{0}}=\frac{\gamma}{2\left(\sigma v_{c}\right)_{T}} \int_{0}^{2} \exp \left(-\frac{x}{2 r_{p}} \gamma\right) \\
& {\left[\int _ { E _ { p , M I N } } ^ { E _ { p , M A X } } f ( E _ { p } ) \left[\int_{0}^{\pi} \sigma_{c x} v_{c} \frac{1}{2} \sin \theta\right.\right.} \\
& \left.\left.x \exp \left(-\frac{y}{2 r_{p}} \frac{v_{0}\left(\sigma v_{c}^{*}\right\rangle_{i}}{v_{p}\left(\sigma v_{c}\right)_{T}} \gamma\right) d \theta\right] d E_{p}\right] d\left(\frac{x}{r_{p}}\right) .
\end{aligned}
$$

The innermost exponential term in the integral of Eq. (C14) is a function of all three variables $E_{p^{x}} \theta$, and $x$, and is the only term which prevents the direct evaluation of the integral. As an approximation, we replace this term by

$$
\exp \left(-\frac{1}{2} \frac{\left\langle a v_{c}\right\rangle_{i}}{\left\langle\sigma v_{c}\right\rangle_{T}}\right)
$$

which then can be removed from the integral. The approximation assumes that the "average $e^{\text {" }} v_{p}$ is equal to $v_{0}$, and the "average" $y$ is equal to $r_{p}$. Substitution of the approximation into Eq. (Cl4) yields

$$
\frac{\mathrm{I}_{c \mathrm{x}}}{\mathrm{I}_{0}} \cong \frac{\left\langle\sigma v_{c}\right\rangle \mathrm{cx}}{\left\langle\sigma v_{c} \mathrm{~T}\right.}\left(\mathrm{I}-\mathrm{e}^{-\gamma}\right) \exp \left(-\frac{\gamma}{2} \frac{\left\langle\sigma v_{c}\right\rangle_{\mathrm{i}}}{\left\langle\sigma v_{\mathrm{c}} \mathrm{T}\right.}\right) .
$$

Equation (C15) was previ.jusly given by Hovingh and Moir. $\mathrm{Cl}$

The complete integration of Eq. (Cl4) was performed numerically for the use of deuterium, and the results are shown as solid curves in Fig. C-3. The approximate Eq. $(\mathrm{Cl})$ has been evaluated for the case of deuterium by Hovingh, ${ }^{\mathrm{C} 3}$ who used $\left\langle\sigma \mathrm{v}_{\mathrm{c}}\right\rangle$ values taken directly from Riviere's curves rather than our approximate formulae. His results are shown as points in Fig. C-3. Also shown in Fig. C-3 (the dashed curves) is the integration of Eq. (Cl4) for the artificial case of no reionization of charge-exchange neutrals ( $y$ was set equal to zero).

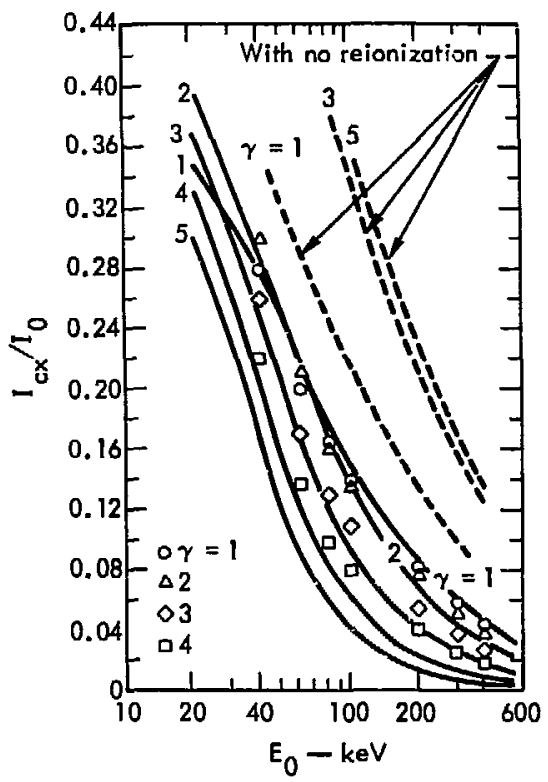

Fig. C-3. Fractional current loss of injected beam due to charge exchange. 
The comparison of the dashed and solid curves of Fig. C-3 reveals the importance of the reionization of the charge-exchange neutrals in reducing the fractional loss of the injected beam. With no reionization, the loss is predicted to increase with $\gamma$, while with reionization the loss decreases with increasing $\gamma$. Although reionization must be included, the good agreement between Hovingh's approximation and the complete integration of Eq. (Cl4) indicates that the approximate representation is adequate for the calculation of the overall loss.

As previously given, the fraction of the injected beam which penetrates entirely through the plasma is

$$
\frac{I_{p}}{I_{0}}=\mathrm{e}^{-\gamma}
$$

The total fractional current loss of the injected beam due to both charge-exchange and penetration is the sum of Eq. (Cl6) and the curves of Fig. C-3. This total fractional loss is shown in Fig. C-4.

Equation ( $\mathrm{C8}$ ) can be rewritten for charge-exchange power, $\mathrm{P}_{\mathrm{cx}}$, by multiplying the right-hand side by the plasma ion energy $\mathrm{E}_{\mathrm{p}}$. The triple integration of this

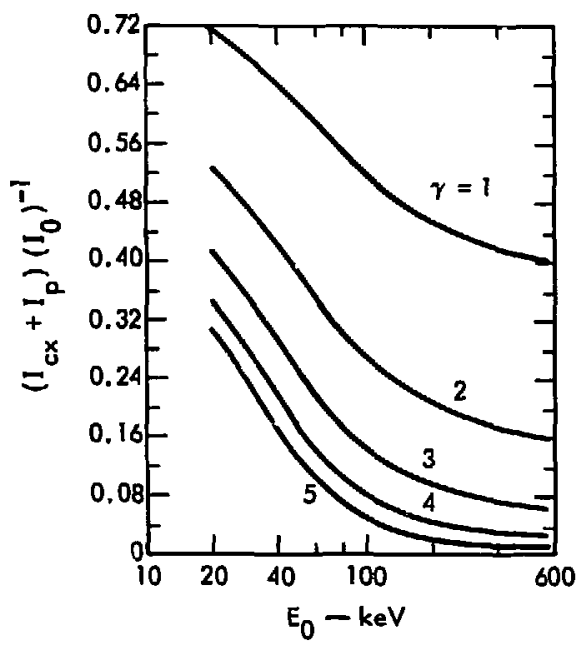

Fig. C-4. Fractional current loss of injected beam due to charge exchange and penetration. new equation over all $\mathrm{E}_{\mathrm{p}}, \theta$, and $\mathrm{x}$ was performed numerically to yield the ratio of $P_{c x}$ to the injected power, $P_{0}=I_{0} E_{0}$. For the present case, $P_{c x} / P_{0}$ is almost equal to $\mathrm{I}_{\mathrm{cx}} / \mathrm{I}_{0}$. The ratio $\left(\mathrm{P}_{\mathrm{cx}} / \mathrm{P}_{0}\right) /\left(\mathrm{l}_{\mathrm{cx}} / \mathrm{I}_{0}\right)$ is shown in Fig. C-5.

To investigate the sjatial distribution of the chargeexchange neutrals which reach the surface of the plasma, we perform two change of variable operations on Eq. (C8). First, we change from the variables $\left(\mathrm{E}_{\mathrm{p}}, \boldsymbol{\theta}, \mathrm{x}\right)$ to $\left(\mathrm{E}_{\mathrm{p}}, \alpha, \mathrm{x}\right)$ by multiplying the right hand side of Eq. (C7) by the Jacobian

$$
J\left(\frac{E_{p}, \theta, x}{E_{p}, \alpha, x}\right)=\frac{\partial \theta}{\partial \alpha}
$$

From Eq. (CS) we derive

$$
\frac{\partial \theta}{\partial \alpha}=\frac{\sin \theta \cos \theta-\cos \alpha+\sin \alpha \sin ^{2} \theta}{\sin \alpha} .
$$

Next, we change from the variables $\left(E_{p}, \alpha, x\right)$ to $\left(E_{p}, s, x\right)$ where ds is an element of the spherical plasma surface; i.e.,

$$
\text { ds }=2 \pi r_{p} \sin \alpha x p d \alpha
$$

This change of variables is accomplished by multiplying the right-hand side of $\mathrm{Eq}$. (C8) by the Jacobian

$$
J\left(\frac{E_{p}, \alpha, x}{E_{p}, s, x}\right)=\frac{\partial \alpha}{\partial s}=\frac{1}{2 \pi r_{p}^{2} \sin \alpha}
$$

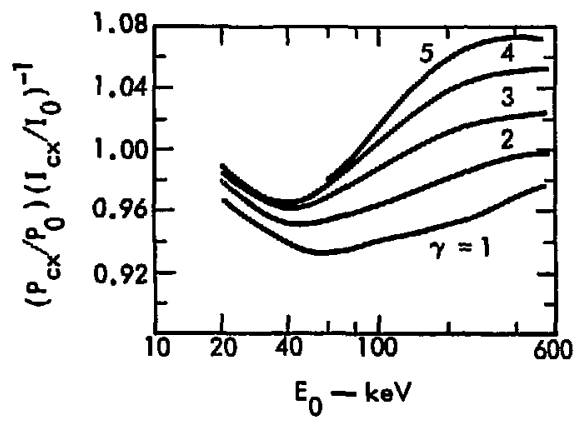

Fig. C-5. Ratio of fractional power loss to fractional current loss due to charge exchange. 
Performing the two change of variable operations in Eq. (C8) yields

$$
\begin{aligned}
& \frac{d^{3} I_{c x}}{d E_{p} d s d\left(x / r_{p}\right)}=\frac{I_{0}}{4 \pi r_{p}^{2}} \frac{\gamma}{2} \frac{\sigma_{c x} v_{c}}{6 y_{c} J_{T}} f\left(E_{p}\right) \\
& x \frac{\sin ^{2} \theta \cos \theta \cos \alpha+\sin ^{3} \sin ^{3} \theta}{\sin ^{2} \alpha} \\
& x \exp \left(-\frac{x}{2 r_{p}} \gamma\right) \exp \left(-\frac{y}{2 r_{p}}-\frac{v_{0}\left(0 v_{c}^{*}\right\rangle_{i}}{v_{p} 6 v_{c} j_{T}} \gamma\right)
\end{aligned}
$$

The double integration of Eq. (C21) over all $E_{p}$ alid $x$ yields the current density of charge-exchange neutrals which reach the surface of the jlasma as a function of injection energy $E_{0}$, attenuation parameter $\gamma$, and angle $\alpha$. The integral is written

$$
\frac{\frac{d I_{c x}}{d s}}{\frac{I_{0}}{4 \pi r_{p}^{2}}}=\frac{\gamma}{2\left(\sigma v_{c}\right)_{T}} \int_{0}^{2} \exp \left(-\frac{x}{2 r_{p}} \gamma\right)
$$$$
x \frac{\sin ^{2} \theta \cos \theta \cos \alpha+\sin \alpha \sin ^{3} \theta}{\sin ^{2} \alpha}
$$$$
x \int_{E_{p, M I N}}^{E_{p, M A X}} f\left(E_{p}\right) \sigma_{c x} v_{c}
$$$$
\left.x \exp \left(-\frac{y}{2 r_{p}} \frac{v_{0}\left(0 v_{c}^{*}\right)_{i}}{v_{p}\left(0 v_{c}\right)_{T}} \gamma\right) d E_{p}\right] d\left(\frac{x}{r_{p}}\right)
$$

The integration of Eq. (C22) was performed numerically for the case of deuterium. Then the results were divided by the previous results for $I_{c x} / I_{0}$ (from the numerical integration of Eq. (C14). The final result is the ratio of local to average current density of charge-exchange neutrals at the surface of the plasma. This ratio is plotted in Figs. C-6 and C-7 as a function of angle $\alpha$ for various values of $\mathrm{E}_{0}$ and $\gamma$.

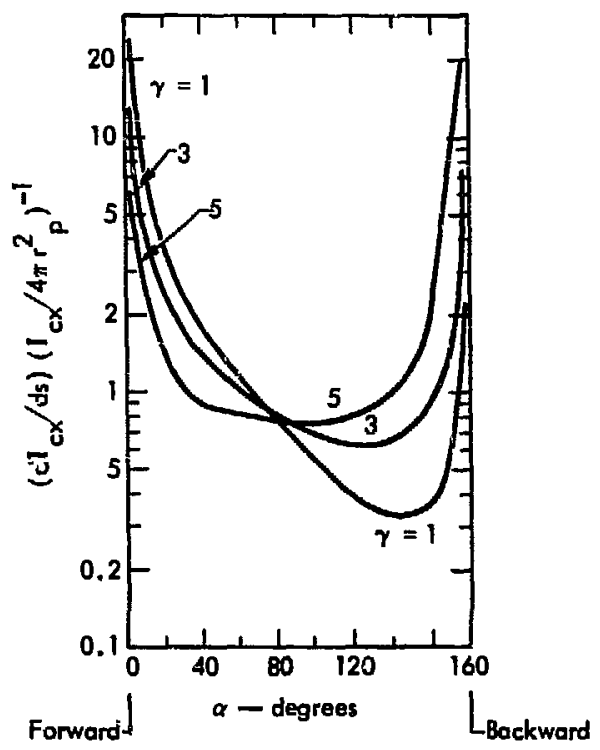

Fig. C.6. Ratio of local in average current density of chargeexchange neulal particles for $E_{0}=65 \mathrm{keV}$.

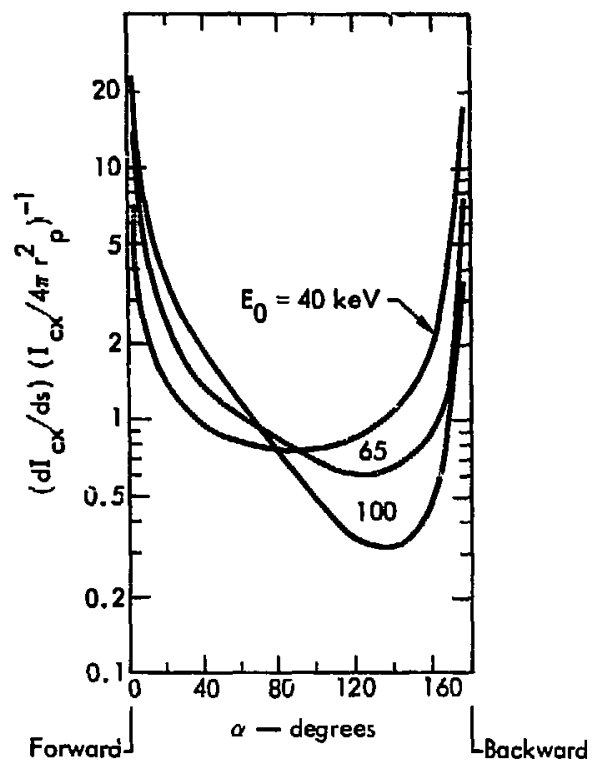

Fig. C.7. Ratio of local to average current density of chargeexchange neutral particles for $\boldsymbol{\gamma}=3$. 
The current density of charge-exchange neutrals is peaked on the injection beam axis, in both the forward and backward directions. The direction of the higher peak depends on the injection energy $E_{0}$ and the attenuation pa:ameter $\gamma$. As shown in Fig. C-6, at constant $\mathrm{E}_{0}=65 \mathrm{keV}$ the higher peak is forward for $\gamma=1$ and 3 and backward for $\gamma=5$. As shown in Fig. C-7, at constant $\gamma=3$ the higher peak is torward for $E_{0}=100$ and $65 \mathrm{keV}$ and backward for $E_{0}=40 \mathrm{keV}$

The current density of charge-exchange neutrals is mathenatically infinite ai both $\alpha=n$ and $\alpha=180^{\circ}$ becasse the injected beam of finite curren: vas assumed to have an infinitesimal cross-sectional area. To estimate the peak-to-average current density ratio of charge-exchange neutrals for a finite area injection beam (but small beann diameter compared to plasma diameter), we assume that the current density at the plasma surface within the beam is uniform and equal to the integrated average from $\alpha=0$ or $180^{\circ}$ to the angle $\alpha_{\mathrm{e}}$ correspunding to the edge of the beam. The average is calculated as follows:

$\left(\frac{\mathrm{dI} \mathrm{cx}}{\mathrm{ds}}\right)_{\text {within beam }}$

$=\frac{\int_{0 \text { or } 180^{\circ}}^{\alpha} \frac{d l_{c x}}{d s} 2 \pi r_{p}^{2} \operatorname{sinad\alpha }}{\int_{0 \text { or } 180^{\circ}}^{\alpha x} 2 \pi r_{p}^{2} \sin \alpha d \alpha}$.

The average current density of charge-exchange neutrals within a finite beam with $E_{0}=65 \mathrm{keV}$ is shown in Fig. C-8 as a function of the angle $\alpha_{\mathrm{e}}$ corresponding to the edge of the beam. Results are shown for both the forward and backward directions. In all cases, the average is somewhat greater than the corresponding local current density at $\alpha=\alpha_{\mathrm{e}}$ for the in linitesimal beam (Fig. C-6), but not much greater because of the sine term in Eq. (C23).

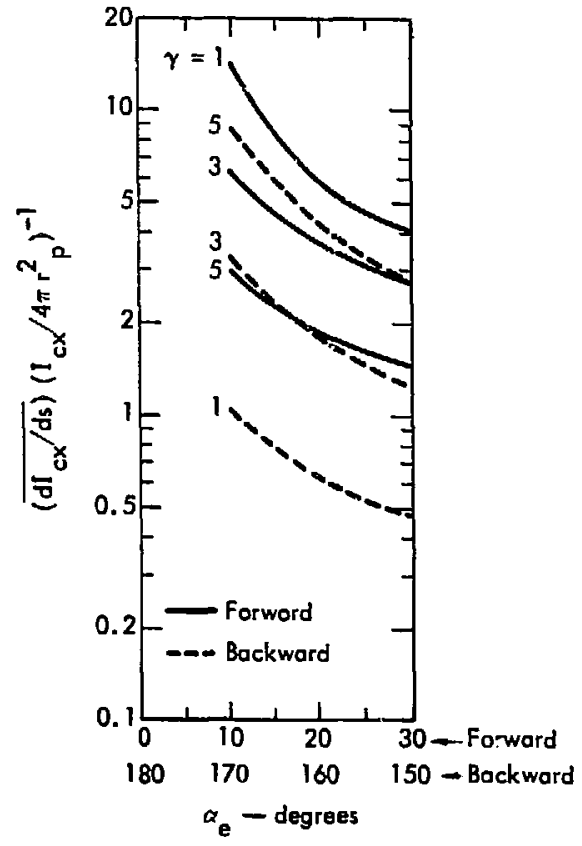

Fig. C-8. Ratio of average curtent density of charge-exchange neutral particles within beam to overall average current density for $E_{0}=65 \mathrm{keV}$.

Equation (C22) can be rewritten for charge-exchange power distribution, $\mathrm{dP}_{\mathrm{cx}} / \mathrm{ds}$, by multiplying the righthand side by the plasma ion energy, $E_{p}$. This new equation was integrated numerically and the results were found to be very similar to those for current distribution. For completeness, Fig. C-9 gives the ratio of local to average power density of chargeexchange neutrals at the surface of the plasma as a function of angle $\alpha$, for $E_{0}=65 \mathrm{keV}$. Figure $C-10$ gives the average power density of charge-exchange neutrals within a finite beam in both the forward and back ward direction.

\section{RESULTS FOR FERF}

The results of the analysis presented in the preceding section are not directly applicable to FERF without additional interpretation because of several complications. Foremost among these is the complex geometry of FERF. FERF has four injectors, two in the vertical plane and two in the horizontal. The individual 


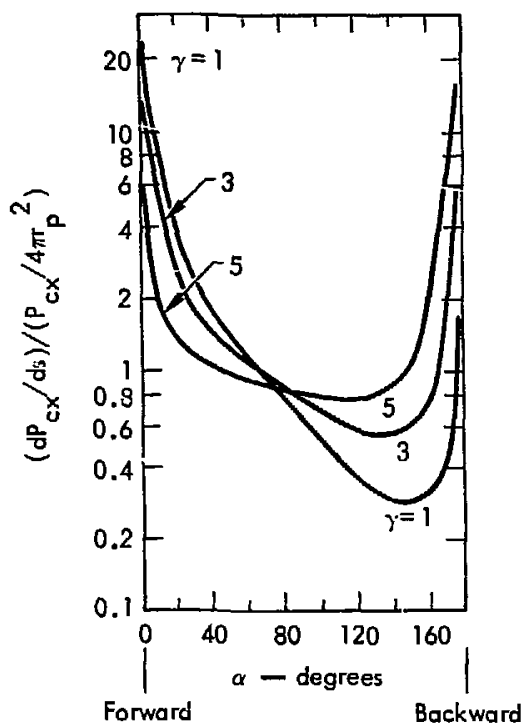

Fig. C-9. Ratio of local to average power density of chargeexchange neutral particles for $E_{0}=65 \mathrm{keV}$.

elentents of each injector are arranged on a curved surface so that the many individual beams of each injector converge to a focus at the injector port (see Fig. 1, page 5 ). Beyond the injector port, the beams diverge. Thus, the cross-sectional area of plasma and furst wall which is illuminated by the injector is considerably more than the area of the injector port. The beam-attenuation parameter varies across the beam because of the density variation of the plasma and the different path lengths through the plasma.

An additional complication in FERF is the injection of deuterium and tritium at the same velocity and hence at different energies (65-keV D and 97.5-keV T). This injection results in a two-humped plasma ion energy distribution. Fokker-Planck calculations have shown the deuterium distribution to be peaked at $65 \mathrm{kcV}$ and the tritiun distribution at $90 \mathrm{keV}$. The speed distributions are very similar for both. Since the collisional processes which we consider are dependent on speed, not energy, we shall assume that the fractions of the beam which charge exchange and ionize in FERF are the same as if the plasma ions were all deuterium and the injection energy was $65 \mathrm{keV}$. This is the case we have considered in the preceding analysis. Of cotrse, when calculating power we must

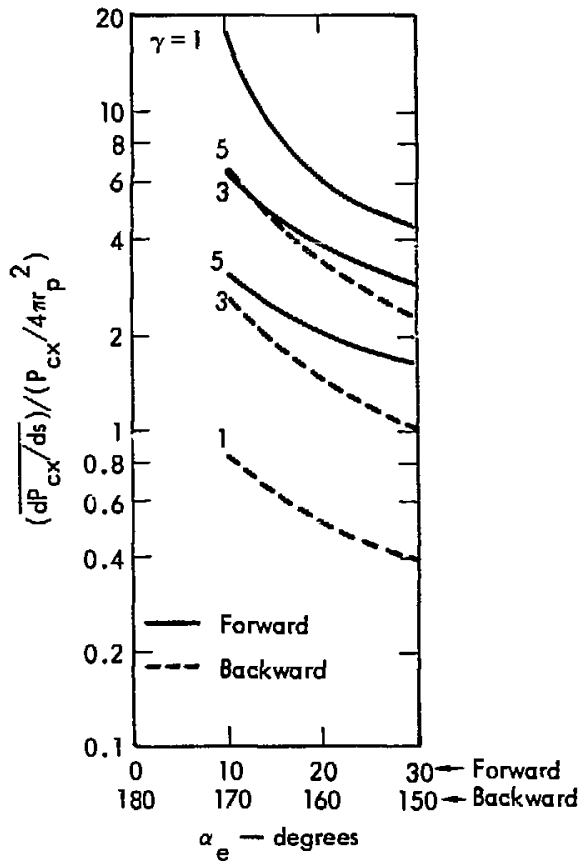

Fig. C-10. Ratio of average power density of charge-exchange neutral particles within beam to overall average current derısity for $E_{0}=65 \mathrm{keV}$.

remember the fraction of high-energy tritium.

\section{Some Geometry Considerations for the Injectors}

Each of the four injectors is capable of injecting $145 \mathrm{~A}$ of $\mathrm{D}^{0}$ and $\mathrm{T}^{0}$ into the plasma chamber (106 A of $65-\mathrm{kV} \mathrm{D} \mathrm{D}^{0}$ and $39 \mathrm{~A}$ of $97.5-\mathrm{keV} \mathrm{T}^{0}$ ). The injected power for each injector is $10.7 \mathrm{MW}$. FERF operation may require simultaneous use of two, three, or even all four injectors. The neutrals from each injector enter the plasma chamber through a 20 -cm square injector port. At the injector port, the current density is $0.363 \mathrm{~A} / \mathrm{cm}^{2}$, and the power density is $26.8 \mathrm{~kW} / \mathrm{cm}^{2}$.

Consider the fath of the injected beam with no plasina present. The area of the far wall which is illuminated by the diverging beam is about $0.81 \mathrm{~m}^{2}$. Therefore, the average power density of: the illuminated portion of the wall due to the ur.ustenuated bearn is about $1.3 \mathrm{~kW} / \mathrm{cm}^{2}$. The maximum power density is at the point on the wall nearest to the injector port and is about $3.3 \mathrm{~kW} / \mathrm{cm}^{2}$. If possible, 
the illuminated portion of the first wall :hould be designed to withstand this thermal load, at least for a

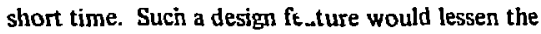
problems of plasma startup and shutdown. The power densities involved are encouragingly close to those which are considered feasible for steady-state cooling of the first wall (see Appendix J). Aside from the thermal load of the unattenuated beam, we must be concerned about wall damage due to the impinging particles. The particle fluxes on the illuminated portion of the first wall are $1.1 \times 10^{17}$ particles/ $\mathrm{cm}^{2} \mathrm{~s}$ average and $2.8 \times 10^{17}$ particles $/ \mathrm{cm}^{2} \mathrm{~s}$ maximum.

\section{Attenuation Parameter in the FERF Plasma}

In the preceding analysis, we have used a plasmaattenuation parameter which can be calculated from Eqs. (C6) and (C7):

$$
\gamma=\frac{\ln \left(\sigma v_{c}\right)_{T}}{v_{0}}
$$

where we have substituted the path length through the plasma, $\ell$, for the more restrictive $2 r$. The average density is to be calculated by

$$
\bar{n}=\frac{n_{0}}{\ell} \int_{0}^{\ell} \frac{n}{n_{0}} d \ell,
$$

where $n_{0}$ is the central plasma density. We have calculated attenuation parameters for FERF using the calculated plasma-density contours and find a variation from $\gamma=\mathbf{2 . 6}$ for the steepest injector beam ray, to $\gamma=3.5$ for the central ray, to $\gamma=6.4$ for the shallowest ray." We shall now spply the preceding analysis to FERF using these attenuation parameters.

*These values of $\gamma$ may be somewhat inaccurate because they were scaled from a $\beta=0.5$ case to a $\beta=0.65$ case simply by multiplying by $0.65 / 0.5$
Total Losses Due to Charge Exchange and Ionization

Table $\mathbf{C}-1$ shows the fractional current and power losses for the three representative injector beam rays. The values are estimated from Figs. C-3, C-4, and C-5. The injection energy, $E_{0}$, is $65 \mathrm{keV}$.

The sums of the charge exchange and penetration losses from Tabit C-I are shown in Fig. C-11 as a function of distance across the injected beam. We integrate under the curves to estimate the total current and power loss from the injected beam. The results are

$$
\left(\frac{l_{c x}+l_{p}}{l_{0}}\right)_{T_{j t}}=0.16
$$

and

$$
\left(\frac{\mathrm{p}_{\mathrm{cx}}+\mathrm{p}_{\mathrm{p}}}{\mathrm{P}_{0}}\right)_{\text {Yot }}=0.16
$$

So, $16 \%$ of both the injected current and injected power in FERF is lost to the first wall. The remaining $84 \%$ of the injected current is trapped in the plasma. For each 145-A injector (recall that FERF operation may require two, three, or even all four injectors to operate simulaneously), 23 A of neutral deuterium and tritium atoms impact the first wall and deposit a total power of $1.7 \mathrm{MW}$. Once the wall is saturated, we can expect the full amperage of deuterium and tritium to be re-emitted as cold gas. The ability of the plasma to tolerate this gas load is discussed in Appendix A.

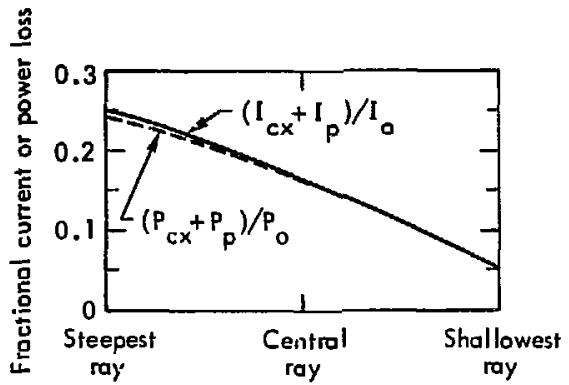

Fig. C-11. Sum of the charge-exchange and penetratic ? losses as a function of distance across the injected bean. 
Table C-1. Fractional current and power losses for representative injector beam rays.

\begin{tabular}{lcccc}
\hline & & $\begin{array}{c}\text { Sreepest ray } \\
\gamma=2.6\end{array}$ & $\begin{array}{c}\text { Central ray } \\
\gamma=3.5\end{array}$ & $\begin{array}{c}\text { Shallowest ray } \\
\gamma=6.4\end{array}$ \\
\hline Charge Exchange: & $\mathrm{I}_{\mathrm{cx}} / \mathrm{I}_{0}$ & 0.171 & 0.134 & $\sim 0.05$ \\
& $P_{\mathrm{cx}} / \mathrm{P}_{0}$ & 0.165 & 0.131 & $\sim 0.05$ \\
Penetration: & $\mathrm{I}_{\mathrm{P}} / \mathrm{I}=\mathrm{P}_{\mathrm{p}} / \mathrm{P}_{0}$ & 0.074 & 0.030 & 0.001 \\
Total: & $\left(\mathrm{I}_{\mathrm{cx}}+\mathrm{I}_{\mathrm{p}}\right) / \mathrm{I}_{0}$ & 0.248 & 0.164 & $\sim 0.05$ \\
& $\left(\mathrm{P}_{\mathrm{cx}}+\mathrm{P}_{\mathrm{p}}\right) / \mathrm{P}_{0}$ & 0.239 & 0.161 & $\sim 0.05$ \\
\hline
\end{tabular}

Current Densities and Power Densities on the First Wall.

We have previously defined the ill'minated portion of the first wall as that portion which is in the direct path of the injected beam. As previously given, the area of the illuminated portion of the first wall for each injector is about $0.81 \mathrm{~m}^{2}$. The average power density on the illuminated portich of the first wall will be somewhat less than

$$
\frac{1.7 \mathrm{MW}}{0.81 \mathrm{~m}^{2}}=210 \frac{\mathrm{W}}{\mathrm{cm}^{2}}
$$

because some of the charge-axchange neutrals impact the first wall outside the illuminated portion. Likewise, the average particle flux density on the illuminated nortion of the first wall will be somewhat less than

$$
\frac{23 \mathrm{~A}}{0.81 \mathrm{~m}^{2}}=1.8 \times 10^{16} \frac{\text { particles }}{\mathrm{cm}^{2} \cdot \mathrm{s}} .
$$

The peak particle flux density and power density will oucur at the point on the first wall illuminated by the steepest injector beam ray (for which $\gamma=2.6$ ). As given previously, the particle flux density at this point with no plasma attenuation $(\gamma=0)$ is $2.8 \times 10^{17}$ particles $/ \mathrm{cin}^{2}$-s and the power density is $3.3 \mathrm{~kW} / \mathrm{cm}^{2}$. With plasma attenuation, the particle flux density and n swer density at this point due to penetration alone is just $\mathrm{e}^{-\gamma}$ times these values, or $2.0 \times 10^{16}$ particles/ $\mathrm{cm}^{2} \mathrm{~s}$ and $250 \mathrm{~W} / \mathrm{cm}^{2}$. The particle flux density and power density at this point due to charge-exchange neutrals cannot be estimated from Figs. C-6 tinrough $C-10$ because the FERF injector beams are nearly as large in cross section as the plasma. a case for which the analysis is invalid. We shall overestimate the peak contribution by charge-exchange neutrals by assum. ing that all charge-exchange neutrals travel in the same direction as the injected neutral. Then, the peak contribution by charge-exchange neutrals is just the unattenuated particle flux density or power density nultiplied by $I_{c x} / l_{0}$ or $P_{c x} / P_{0}$ for $\gamma=2.6$ (îrom Table $(-1)$. The results are a peak particle flux density of $4.8 \times 10^{16}$ particles $/ \mathrm{cm}^{2} s$ and a peak power density of $540 \mathrm{~W} / \mathrm{cm}^{2}$. Adding the contributions due to penetration and charge-exchange neutrals yields a peak neutral-particle flux density of $6.8 \times 10^{16}$ particles $/ \mathrm{cm}^{2} \mathrm{~s}$ and a peak power density of 790 $\mathrm{W} / \mathrm{cm}^{2}$.

Table $\mathrm{C}-2$ summarizes the predicted bombardment of the first wall of FERF by neutral atoms. 
Table C-2. Summary of FERF first-wall bombardment.

Amps injected $=145$ A per injector

(106 A of $65 \mathrm{keV} \% \mathrm{D}^{0}$ )

( $35 \mathrm{~A}$ of $97.5 \mathrm{keV} \% \mathrm{~T}^{0}$ )

Power injected $=10.7 \mathrm{MW}$ per injector

Area of first waj illuminated by each injector $=0.81 \mathrm{~m}^{2}$

With no plasma

Amps to the wall $=145 \mathrm{~A}$ for each injector

Power to the wall $=10.7 \mathrm{MW}$ for each injecter

Average particle flux density to wali

$=1.1 \times 10^{17}$ particles $/ \mathrm{cm}^{2} \cdot \mathrm{s}$

Peak particle flux density to wall

$=2.8 \times 10^{17}$ particies $/ \mathrm{cm}^{2}$.s

Average power density to wall $=1.3 \mathrm{~kW} / \mathrm{cm}^{2}$

Peak power density to wall $=3.3 \mathrm{~kW} / \mathrm{cm}^{2}$

With plasma

Amps to the wall $=23 \mathrm{~A}$ for each injector

Power to the wall $=1.7 \mathrm{MW}$ for each injector

Average particle flux density to wall

$<1.8 \times 10^{16}$ particles $/ \mathrm{cm}^{2} \cdot \mathrm{s}$

Peak particle flux density to wal!

$<6.8 \times 10^{16}$ particles $/ \mathrm{cm}^{2}$.s

Average power density to wall $<210 \mathrm{~W} / \mathrm{cm}^{2}$

Peak power density to wall $<790 \mathrm{~W} / \mathrm{cm}^{2}$

\section{REFERENCES}

C1. J. Hovingh and R. W. Moir, Efficiency of Injection of High Energy Neutral Beams into Thermonuclear Reactors, LLL, Rept. UCRL-51419 (1973).

C2. A. C. Riviere, Nuclear Fusion 11, 363 (1971).

C3. J. Ilovingh, LLL, private communication (1973). 


\section{Appendix D - Shieldi Analysis}

\section{INTRODUCTION}

This section summarizes the results of a preliminary study of a shield design for the FERF. The calculation method and results will also bc useful for the design and construction of other types of reactors.

The principle result is the amount of heat deposited in the superconducting magnet coils. Since these coils run at cryogenic temperatures, and since the required refrigeration systems have a low efficiency $(\sim n .1 \%)$, sufficient shielding is required to minimize the consumption of electrical power by the refrigerator. Additional results are neutron and gamma fluxes throughout the system during operation, heating in other components, buildup of radioisotopes produced during operation, and the dose rates from these radioisotopes after siutdown.

It should be stressed that the purpose of these calculations is to show the feasibility of a FERF design and to bring to light potential problem areas. insufficient effort has been expended to design a shield of optimum behayior or to assess the shielding impact upon other parts of the design. Although foar separate system-geometry design'; were made, several more designs are required for proper consideration of all possible point $s$ of interaction. Many more cases must beconsidered to arrive at an "optimum" system. In particulas, shield materjals should be considered which will reduce the rtsidual radionctivity.

\section{CALCULATIONAL TECHNIQUES}

The majority of the neutron and gamma transport problems solved in this study made use of the MORSE-L* Mante Carlo code. This code makes use of multigroup techniques to simulate the energy dependence of the transport problem and is able to describe coupled neutron-gamma problems as well as all-neutron and all-gamma problems. A generalized, three-dimensional geometry routine is employed to describe the system. Special biasing and exponential transform techniques are available, and were used, to

*MORSE-L is an LLL version of the MORSE ${ }^{\mathrm{Dl}}$ code originally developed at ORNL. solve the deep penetration problems encountered in this study. A few problems made use of the ANISN$L^{* *}$ one-dimensional, discrete-ordinates code.

Basic cross-section data used in both these codes were obtained from the following sources:

- Neutron data were obtained from the standard LLL evaluated cross section library $\mathrm{D3}$.

- Gamma data cross sections were obtained from the Plechaty-McMaster compilation ${ }^{\mathrm{D} 4}$ and scatlering from the standard Klein-Nishima equations.

- Gamma production data were based upon experimental spectra and the LLL evaluated neutron cross sections and were normalized to conserve gamma energy.

The 30 neutron energy groups used spanned a energy range of $18 \mathrm{MeV}$ to thermal. Fifteen gamma groups, from $10 \mathrm{MeV}$ to $0.5 \mathrm{MeV}$, were employed. The neutron cross sections used in this study have also been used to calculate all the LLL pulsed-sphere experiments. D5 The results have been satisfactory for all cases. The gamma data have been employed in the solution of benchmark gamma problems with good success. A few coupled neutron-gamma problems have been solved with reasonable accuracy out there is, in general, a lack of such benchmark problems.

Activation cross-section data were obtained from the compilation of Alley and Lessfer $\mathrm{D} 6$ and averaged over the neutron group structure. Decay gamma energies and intensities were taken from the sixth edition of the Table of Isotopes. ${ }^{D 7}$ It should be noted that activations have been calculated only for those isotopes which are gamma emitters and only gammas greater than $0.5 \mathrm{MeV}$ have been considered.

\section{GEOMETRICAL MODEL}

It is evident that the heating in the magnet coils, as weli as other quantities dependent on neutron and gamma fluence, is very sensitive to the geometry of the system. The FERF geometry cannot be adequately described by one- or two-dimensional models with

**ANISN-L is an LLL version of the ANISN ${ }^{D 2}$ code. 
any assurance that the results will be meaningful. For this reason, a full, three-dimensional description was made of the FERF system. The model used followed the designed layout as closely as possible with limits imposed only by the available computer routines and by a limited time schedule.

Figure D-1 illustrates a cross section of a single magnet section as described for the shielding analysis. This is a cut through one magnet section at its center. The full magnet is formed by rotating this section 80 degrees each side of this point (toward and aw ny from the viewer), with the outermost surfaces rotated about a point $80 \mathrm{~cm}$ behind the center of the system and the innermost rotated about the center. At the 80 degree point, the magnet section "rolls" around a fixed axis into a similar magnet section located below the first to form a complete magnet. A second, similar magnet is positioned at right angles to and to the left of the first. Figure D-2 illustrates a cut through the entire model at its center plane. Shielding is added between the magnets to fill part of the volume that normally would be left with a void gap as illustroted on this figure. Four conical holes are cut into the resulting model to simulate the rectangular holes cut to admit particles from the ion sources. The ion sources themselves are simulated by four $75-\mathrm{cm}-$ thick conical frustums, located in each of these cones at positions roughly approximating the designed ion sources. The entire machine is placed in the exact center of a zoom 100-feet long by 60 -feet wide by 60 -feet high. The walls of the room are formed of a 2 -cm-thick steel liner and then 3 feet of concrete.

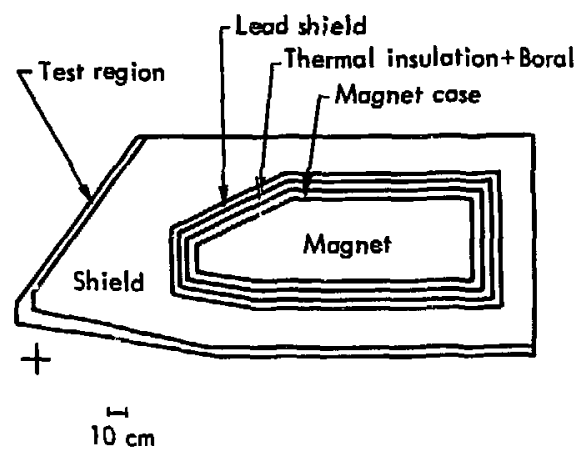

Fig. D-1. Basic geometry used in the shielding calculations.

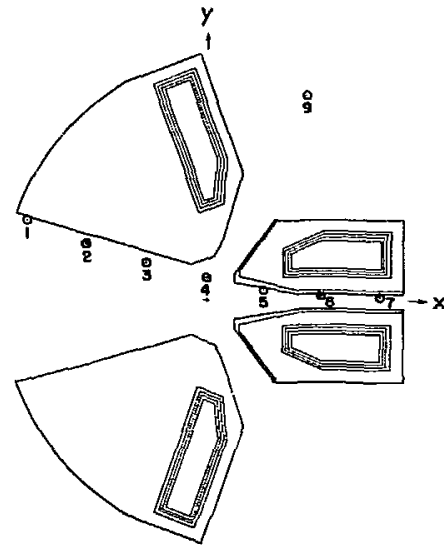

o8

Fig. D-2. Cross-sectional view of the geometry model used for shielding calculations ( $\mathrm{z}=0$ plane).

Several approximations have been made in forming this model:

- The test region, which includes the first wall and the structure to hold the test specimens (but not the specimens themselves), has been omitted in several places. (Fig. D-2 does not show this region in places where it should be.) In general, the volume of the omitted region is smill compared to the volume included in the problem.

- As mentioned, the holes for the ion sources are circular rather than rectangular.

- Figure D-1 illustrates a slight volume removed from the left side of the bottom of the magnet. The designed system has a flat bottow. The extra cut made it possible to use an existing model with minimum perturbation (necessary because of a lack of time). Very little error should result from this change.

- The physical struclure (C-clamp) which holds the magnet together has been omitted. This is outside of the described geometry and will not alter the magnet heating. Since it is in a position where no direct beams of unattenuated neutrons hit it, its presence will not appreciably affect the shutdown dose rates.

- The structure which holds the two magnets apart from each other was not included. As illustrated on at least one drawing, this volume could be part of the cryogenic system and could alter the radiation heating 
results. It is not completely clear what this volume would look like.

- Small portions of the shield at the outer part of the "roll" sections (away from the source) have been omitted. These should not change in the calculated results.

- Items external to the described system have been omitted. These will alter the dose rate after shut down and the calculated activation.

Figure D-3 is a computer-drawn view of the geometry and illustrates the various materials found on the faces of a box cutting through the defined geometry. A cut into the box is made to show the inside of the system. Several dozen different pictures of the system were drawn to insure that the computer model correctly duplicates the designed system.

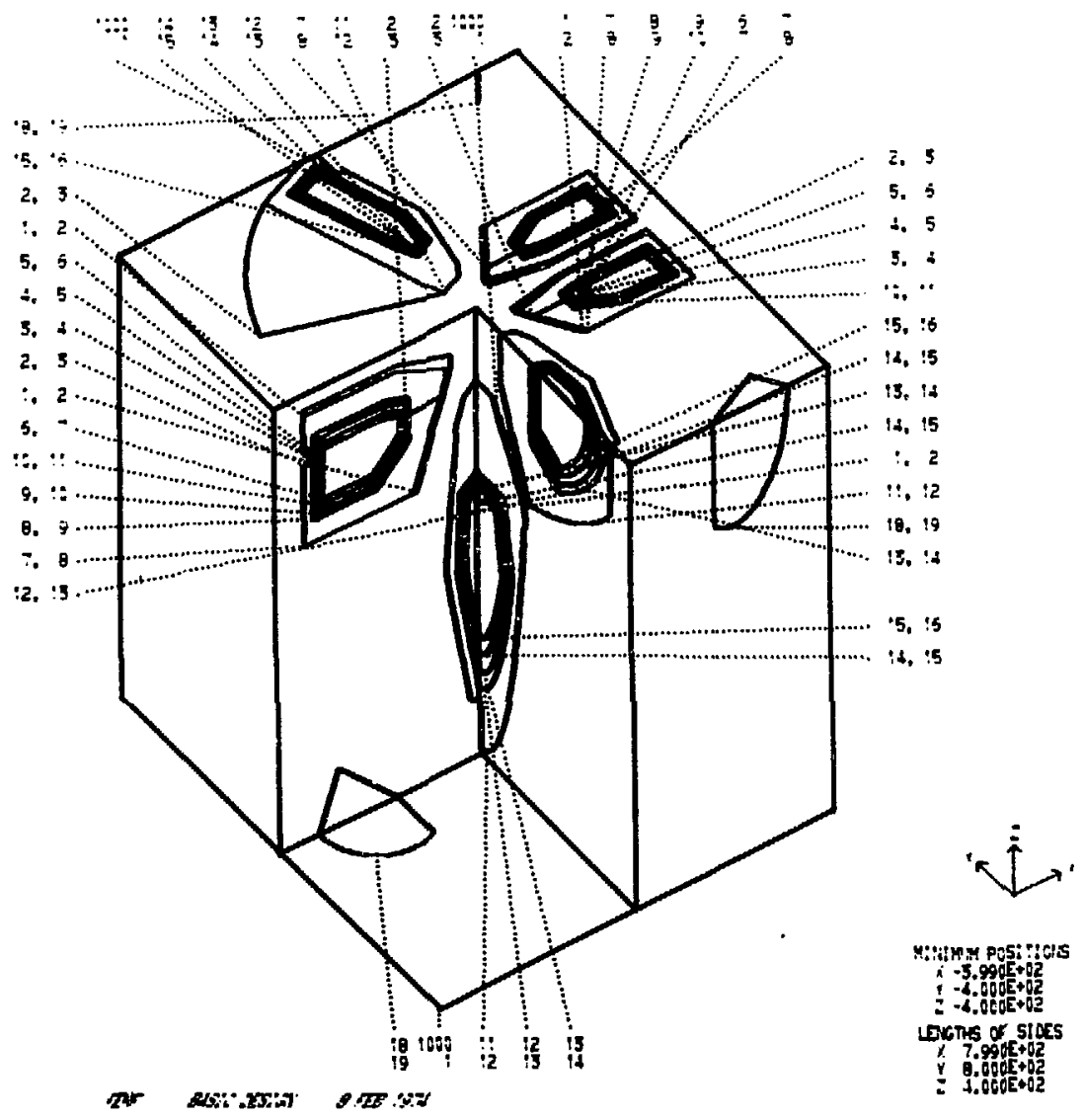

Fig. D-3. Computer illustration of geometry model used in shielding calculations. Numbers refer to material compositions and statistical regions. 


\section{MATERIALS SELECTED FOR MODEL}

Table D-l shows the compositions used for each of the regions of the problem. Note that the region labeled "thermal insulation" is really the sum of a 5-cm-thick region containing thermal insulation and a region containing a sheet of 0.25 -inch-thick Boral. The two matcrials are mixed homogeneously into the one regiol:

The composition of the main shield was chosen after a one-dimensional study of the energy deposition in a magnet located behind a water/steel shield. Figure D-4 illustrates the geometry used in this preliminary one-dimensional study as well as results obtained when the ratio of water-to-steel was varied. This stu-iy produced an optimum shield composition of $80 \mathrm{vol} \%$ steel/ $20 \mathrm{vol} \%$ water, and this same composition was used in the FERF study. It is expected that this may not be an optimum composition when considerations such as minimum activation and cost are studied in future designs.

In the activation of aluminum and lead, the dose rates calculated for a long time after irradiation will be the result of small impurities in the material. Tables D-2 and D-3 indicate the impurities used in
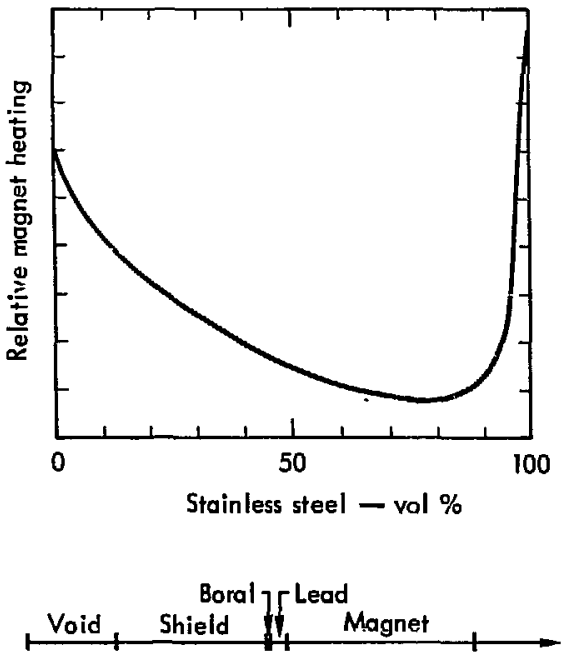

Radial position this study. Note that in practice, the impurities present may vary a great deal from those listed in any handbook tabulation.

The initial source distribution of fusion neutrons in space was designed so that it would adequately simulate the distribution existing in the FERF machine. The source was broken into 12 pieces of equal length along the $\mathrm{x}$ axis, and each of these pieces was then simulated by four concentric rectangular boxes. The source within each box was set as a fixed number per unit volume. By specifying the dimensions of each of the 48 resulting boxes and the number of source neutrons generated in each, it was possible to simulate the actual source. Figure D-5 illustrates the outermost dimensions of the boxes while Table $D-4$ gives the complete description.

The source was normalized so that the uncollided 14-MeV neutron fluence at the first wall at a point closest to the center of the machine (a point located at $[0,40,0]$ with the coordinators used) would be $10^{14} \mathrm{n} / \mathrm{cm}^{2} \mathrm{~s}$. This resulted in a total integrated source of $3.00 \times 10^{18} \mathrm{n} / \mathrm{s}$.

\section{RESULTS OF ANALYSIS}

\section{Energy Deposition}

Table D-5 indicates the energy deposition in various parts of the FERF system. It should be noticed that
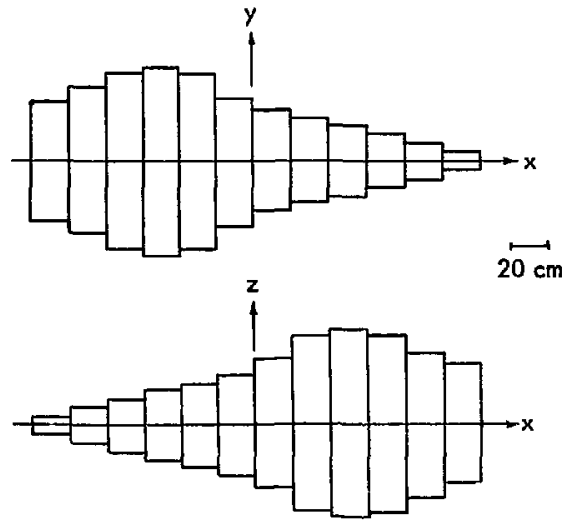

Fig. D-5. Outermost limits of source distribution.

Fig. D-4. Geometry used in une-dimensional study. 
Table D-1. Regional compositions as used in shielding calculations.

\begin{tabular}{|c|c|c|c|c|}
\hline Region & Definition & & $\begin{array}{l}\text { Composition } \\
(\mathrm{at} / \mathrm{b}-\mathrm{cm})^{\mathrm{a}}\end{array}$ & $\left(\mathrm{g} / \mathrm{cm}^{3}\right)$ \\
\hline \multirow[t]{3}{*}{ Test } & 50 vol \% SAP-895 & A] & 0.0294 & \\
\hline & 10 vol $\% \mathrm{H}_{2} \mathrm{O}$ & c. & 0.00258 & \\
\hline & 40 vol $\%$ void & $\mathrm{H}_{2} \mathrm{O}$ & & 0.1 \\
\hline \multirow[t]{2}{*}{ Shield } & 80 vol $\%$ SS304 & SS304 & & 6.336 \\
\hline & $\begin{array}{l}20 \text { vol } \% \mathrm{H}_{2} \mathrm{O} \text { with } \\
6.35 \mathrm{~g} / 100 \mathrm{~cm}^{3} \text { of } \mathrm{H}_{3} \mathrm{BO}_{3} \text { added }\end{array}$ & $\begin{array}{l}\mathrm{H}_{2} \mathrm{O} \\
\mathrm{B}\end{array}$ & $1.24 \times 10^{-4}$ & 0.2 \\
\hline Lead shield & 100 vol \% Pb & $\mathrm{Pb}$ & 0.033 & \\
\hline \multirow[t]{3}{*}{ Thermal insulation } & 11.3 vol $\%$ Boral $\left(\mathrm{B}_{4} \mathrm{C}\right.$ in & $\mathbf{A l}$ & 0.00456 & \\
\hline & Al, clad with Al) & B & 0.00365 & \\
\hline & 88.7 vol $\%$ void (thermal insulation neglected) & $\mathrm{C}$ & $0.0009\lfloor 4$ & \\
\hline Magnet case & 100 vol \% SS304 & SS304 & & 7.92 \\
\hline \multirow[t]{4}{*}{ Magnet } & $6.8 \mathrm{vol} \% \mathrm{Nb}_{3} \mathrm{Sn}$ & $\mathrm{Cu}$ & 0.07578 & \\
\hline & 3.2 vol \% $\mathrm{NbTi}$ & $\mathrm{Nb}$ & 0.00379 & \\
\hline & 90.0 vol $\% \mathrm{Cu}$ & Sn & 0.000877 & \\
\hline & & $\mathrm{Ti}$ & 0.00116 & \\
\hline \multirow[t]{2}{*}{ Ion sources } & 10 vol $\%$ SS304 & SS304 & & 0.792 \\
\hline & 90 vol \% roid & & & \\
\hline SS wall liners & 100 vol \% SS304 & SS304 & & 7.92 \\
\hline \multirow[t]{10}{*}{ Concrete walls } & 100 vol \% concrete & $\mathrm{H}$ & 0.01386 & \\
\hline & & $\mathrm{C}$ & 0.00012 & \\
\hline & & 0 & 0.04582 & \\
\hline & & $\mathrm{Na}$ & 0.001 & \\
\hline & & $\mathrm{Mg}$ & 0.00011 & \\
\hline & & $\mathbf{A}$ & 0.00174 & \\
\hline & & $\mathrm{Si}$ & 0.01063 & \\
\hline & & $\mathbf{K}$ & 0.00046 & \\
\hline & & $\mathrm{Ca}$ & 0.00152 & \\
\hline & & $\mathrm{Fe}$ & 0.00035 & \\
\hline
\end{tabular}

$a_{a t} / b \cdot c m \equiv$ atoms $/$ bam $\cdot \mathrm{cm}$, where 1 barn $=10^{-24} \mathrm{~cm}$.

${ }_{\mathrm{SS} 304}$, at a density of $7.92 \mathrm{~g} / \mathrm{cm}^{3}$, is assumed to have the following composition:

$\begin{array}{cl}\text { Element } & \begin{array}{c}\text { Composition } \\ (\mathrm{at} / \mathrm{b} \cdot \mathrm{cm})\end{array} \\ \mathrm{C} & 0.0001584 \\ \mathrm{Si} & 0.000792 \\ \mathrm{Cr} & 0.017424 \\ \mathrm{Mn} & 0.000792 \\ \mathrm{Fe} & 0.05972 \\ \mathrm{Ni} & 0.00792\end{array}$


the shield has been broken into three parts: the flat sections (Fig. D-1) within $62 \mathrm{~cm}$ of the centerlines (including the filler between the magnets) which is called the "inside;" the flat sections beyond $62 \mathrm{~cm}$ of the centerline which is called the "ousside;" and the

Table D-2. Aluminum impurities (Alcoa 1199).

\begin{tabular}{|c|c|}
\hline Element & ppm (by weight) \\
\hline $\mathbf{S i}$ & 30 \\
\hline $\mathrm{Fe}$ & 10 \\
\hline $\mathrm{Mn}$ & 10 \\
\hline $\mathrm{Mg}$ & 1 \\
\hline $\mathrm{Pb}$ & 1 \\
\hline $\mathrm{Cu}$ & 1 \\
\hline $\mathrm{Cr}$ & 3 \\
\hline ?n & 14 \\
\hline $\mathrm{Ti}$ & 2 \\
\hline$v$ & 3 \\
\hline $\mathrm{Sn}$ & 2 \\
\hline $\mathrm{Bi}$ & 2 \\
\hline B & 4 \\
\hline $\mathrm{Ga}$ & 10 \\
\hline $\mathrm{Zr}$ & 3 \\
\hline $\mathrm{Co}$ & $<2$ \\
\hline Mo & $<2$ \\
\hline $\mathrm{Na}$ & $<1$ \\
\hline $\mathrm{Ca}$ & $<1$ \\
\hline
\end{tabular}

"roll" sections where the two flat sections of the magnet roll into each other.

Allowing for a $20^{\circ} \mathrm{C}$ rise in the temperature of the shield cooling water, flow rates of about $5 \times 10^{4} \mathrm{~g} / \mathrm{s}$ would be required. This would change the water in the shield approximately once per minute. There is adequate space available in the shield and in the test region for the required cooling.

The calculations producing the above results were biased to produce valid results in the magnet region. They also provided fluxes averaged over the various regions of the problem. Additional calculations were performed to obtain results at fixed point detector locations. Table D-6 indicates nine of these locations. The first seven are within the magnets and approximately on the first wall. Point eight is on the extreme outer location of the plasma, outside the magnets, while point nine is on the inside of an ion source.

Figure D-2 indicates these positions.

Table D-3. Lead impurities (desilverized lead)

\begin{tabular}{cc}
\hline Element & ppm (by weight) \\
\hline $\mathrm{Ag}$ & 20 \\
$\mathrm{Cu}$ & 25 \\
$\mathrm{Fe}$ & $\leqslant 20$ \\
$\mathrm{As}-\mathrm{Sn}-\mathrm{Sn}$ & $\leqslant 150$ \\
Zn & $\leqslant 20$ \\
$\mathrm{Bi}$ & $\leqslant 2500$
\end{tabular}

Table D-4. Neutron source distributions. ${ }^{\mathrm{a}}$

\begin{tabular}{|c|c|c|c|c|c|c|c|c|c|}
\hline \multirow{2}{*}{$\begin{array}{l}\text { X-position } \\
\text { (cm) }\end{array}$} & \multirow{2}{*}{$\begin{array}{l}\text { Integrated source } \\
(\mathrm{n} / \mathrm{s})\end{array}$} & \multicolumn{8}{|c|}{ Half-width and half-height of source boxes (cm.) } \\
\hline & & $\mathbf{A}_{1}$ & $\mathbf{B}_{1}$ & $\mathbf{A}_{2}$ & $\mathbf{B}_{2}$ & $\mathbf{A}_{3}$ & $\mathbf{B}_{3}$ & $\mathbf{A}_{4}$ & $\mathbf{B}_{4}$ \\
\hline 0 to 20 & $178 \times 10^{17}$ & 26.9 & 30.6 & 21.5 & 24.5 & 13.5 & 15.3 & 8.1 & 9.2 \\
\hline 20 to 40 & $4.50 \times 10^{17}$ & 22.6 & دo.6 & 18.1 & 30.9 & 11.3 & 19.3 & 6.8 & 11.6 \\
\hline 40 to 60 & $3.43 \times 10^{17}$ & 18.4 & 46.4 & 15.7 & 37.1 & 9.2 & 23.2 & 5.5 & 13.9 \\
\hline 60 to 90 & $1.68 \times 10^{17}$ & 14.1 & 49.6 & 11.3 & 39.7 & 7.1 & 24.8 & 4.2 & 14.9 \\
\hline 80 to 100 & $0.51 \times 10^{17}$ & 9.9 & 46.3 & 7.9 & 37.0 & 5.0 & 23.2 & 3.0 & 13.9 \\
\hline 100 to 120 & $0.10 \times 10^{17}$ & 5.6 & 33.4 & 4.5 & 26.7 & 2.8 & 16.7 & 1.7 & 10.0 \\
\hline
\end{tabular}

${ }^{3}$ Eleven percent of the integrated source is inside box 1 and outside box 2,27.9\% inside box 2 and outside box 3 , $33.5 \%$ inside box 3 and outside box 4 , and $27.6 \%$ inside box 4 . The source description for negative values of $X$ is identical except that the A's and B's are interchanged. 
Table D-5. Operational energy depositions caused by neutron and gamma reactions.

\begin{tabular}{|c|c|c|}
\hline \multirow[b]{2}{*}{ Region } & \multicolumn{2}{|c|}{ Energy Deposition } \\
\hline & $\begin{array}{c}\text { (MeV/source } \\
\text { neutron })^{a}\end{array}$ & $(\mathrm{~kW})$ \\
\hline Test & $2.440(0.0136)$ & $1173 \pm 16$ \\
\hline \multicolumn{3}{|l|}{ Shield: } \\
\hline flat section--inside & $8.192(0.005)$ & $3937 \pm 20$ \\
\hline flat section-outside & $0.922(0.025)$ & $443 \pm 11$ \\
\hline roll section & $4.282(0.018)$ & $2058 \pm 37$ \\
\hline Lead shield & $0.025(0.189)$ & $12.1 \pm 2.3$ \\
\hline Thermal insulation & $0.0057(0.158)$ & $2.76 \pm 0.44$ \\
\hline Magnet cases & $0.0029(0.170)$ & $1.41 \pm 0.24$ \\
\hline Magnets & $0.0079(0.110)$ & $3.82 \pm 0.42$ \\
\hline lon sources & $0.0787(0.126)$ & $37.8 \pm 4.8$ \\
\hline SS wall liners & $0.311(0.080)$ & $150 \pm 12$ \\
\hline Concrete walls & $0.979(0.083)$ & $471 \pm 39$ \\
\hline Total & 17.246 & 8290 \\
\hline
\end{tabular}

${ }^{a}$ Values in parentheses are the fractional standard deviation (one standard deviation divided by the quantity calculated) associated with the given result. These errors represent statistical variations in the Monte Carlo techniques used and do not include data uncertainties.

\section{Neutron and Gamma Fluxes}

Integrated neutron fluxes at the detectors are listed in Table D-7. The statistics associated with these results are poor (probably believable within only a factor of two). Since the statistics are much better for the results of the energy deposition calculation, the average fluxes in the test region were normalized to point four and are also shown in Table D-7. The neutron fluence and the gamma fluence are shown as functions of energy for the test region in Figs.

D-6 and $D-7$, respectively.

Table D-6. Point detector positions.

\begin{tabular}{crrr}
\hline & \multicolumn{3}{c}{ Coordinates (cm) } \\
\cline { 2 - 4 } Detector no. & \multicolumn{1}{c}{$x$} & \multicolumn{1}{c}{$y$} & z \\
\hline 1 & -300 & 130 & 0 \\
2 & -200 & 95 & 0 \\
3 & -100 & 65 & 0 \\
4 & 0 & 40 & 0 \\
5 & 100 & 20 & 0 \\
6 & 200 & 10 & 0 \\
7 & 300 & 10 & 0 \\
8 & 630 & 0 & 0 \\
9 & 180 & 340 & 0 \\
\hline
\end{tabular}

Table D-7. Integrated neutron fluxes at point detectors.

\begin{tabular}{|c|c|c|c|c|c|}
\hline \multirow{2}{*}{$\begin{array}{c}\text { Detector } \\
\text { no. }\end{array}$} & \multicolumn{5}{|c|}{ Neutron flux $\left(\mathrm{n} / \mathrm{cm}^{2} \cdot \mathrm{s}\right)$} \\
\hline & Uncollided & $>10 \mathrm{MeV}$ & $>5 \mathrm{MeV}$ & $>1 \mathrm{MeV}$ & Total \\
\hline 1 & $2.2 \times 10^{12}$ & $2.8 \times 10^{12}$ & $3.0 \times 10^{12}$ & $3.4 \times 10^{12}$ & $4.6 \times 10^{12}$ \\
\hline 2 & $5.3 \times 10^{12}$ & $8.5 \times 10^{12}$ & $9.6 \times 10^{12}$ & $2.7 \times 10^{13}$ & $5.7 \times 10^{13}$ \\
\hline 3 & $2.2 \times 10^{13}$ & $2.6 \times 10^{13}$ & $2.7 \times 10^{13}$ & $4.0 \times 10^{13}$ & $8.1 \times 10^{13}$ \\
\hline 4 & $1.0 \times 10^{14}$ & $1.1 \times 10^{14}$ & $1.2 \times 10^{14}$ & $1.3 \times 10^{14}$ & $2.0 \times 10^{14}$ \\
\hline $\operatorname{ATR}^{\mathrm{a}}$ & $1.0 \times 10^{14}$ & $1.4 \times 10^{14}$ & $1.5 \times 10^{14}$ & $2.3 \times 10^{14}$ & $5.3 \times 10^{14}$ \\
\hline 5 & $4.9 \times 10^{13}$ & $5.4 \times 10^{13}$ & $5.9 \times 10^{13}$ & $7.5 \times 10^{13}$ & $1.1 \times 10^{14}$ \\
\hline 6 & $7.0 \times 10^{12}$ & $8.3 \times 10^{12}$ & $2.0 \times 10^{13}$ & $3.0 \times 10^{13}$ & $4.7 \times 10^{13}$ \\
\hline 7 & $2.6 \times 10^{12}$ & $3.1 \times 10^{12}$ & $3.3 \times 10^{12}$ & $3.7 \times 10^{12}$ & $5.6 \times 10^{12}$ \\
\hline 8 & $4.7 \times 10^{11}$ & $5.0 \times 10^{11}$ & $5.1 \times 10^{11}$ & $5.6 \times 10^{11}$ & $8.1 \times 10^{11}$ \\
\hline 9 & $7.2 \times 10^{11}$ & $8.6 \times 10^{11}$ & $8.9 \times 10^{11}$ & $9.8 \times 10^{11}$ & $1.3 \times 10^{12}$ \\
\hline
\end{tabular}

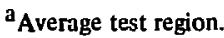




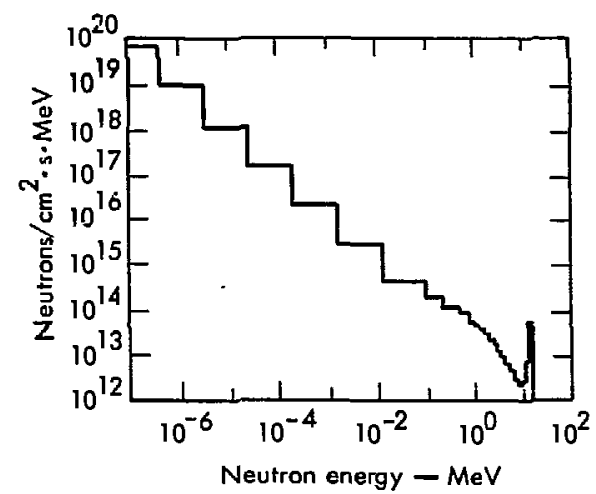

Fig. D-b. Energy-dependent average neutron fluence in test region normalized to an uncollided value of $10^{14} \mathrm{n} / \mathrm{cm}^{2} \cdot \mathrm{g}$.

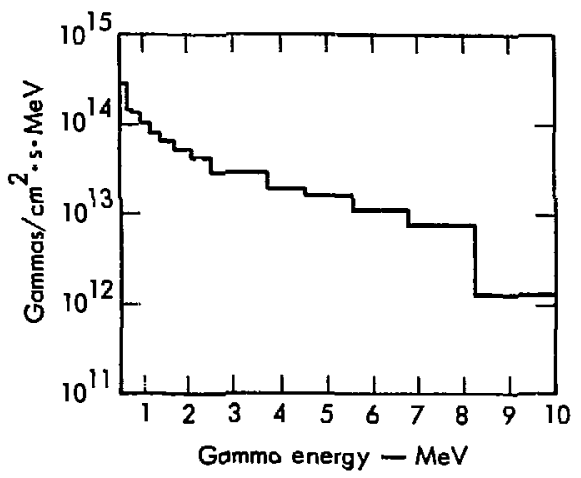

Fig. D-7. Energj-üeprendent garmma fluence in test region normalized to an uncollided neutron value of $10^{14} \mathrm{n} / \mathrm{cm}^{2} \mathrm{~s}$.

Table D-8 gives the biological dose rate at the nine point detectors as well as the heating that would be caused at these points in a very small volume of SAP and stainless steel (small enough to not perturb the fluxes). Additional calculations at points outside the machine indicate dose rates from $10^{5}$ to $10^{7} \mathrm{rem} / \mathrm{h}$ for places where the neutron source cannot be directly seen.

The uncollided 14-MeV neutron flux at a point within the magnet where this flux would be maximum
Table D-8. Calculated quantities at point detectors.

\begin{tabular}{cllll}
\hline & \multicolumn{2}{c}{$\begin{array}{c}\text { Dose rates } \\
\text { (rem/h) }\end{array}$} & \multicolumn{2}{c}{$\begin{array}{c}\text { Neutron and } \\
\text { gamma heating } \\
\text { rates }\left(W / \mathrm{cm}^{3}\right)\end{array}$} \\
$\begin{array}{c}\text { Detector } \\
\text { no. }\end{array}$ & Neutron & Gamma & SAP & SS \\
\hline 1 & $7.2 \times 10^{8}$ & $2.7 \times 10^{6}$ & 0.1 & 0.2 \\
2 & $5.0 \times 10^{9}$ & $1.0 \times 10^{8}$ & 1.3 & 3.3 \\
3 & $8.3 \times 10^{9}$ & $1.1 \times 10^{8}$ & 1.7 & 3.9 \\
4 & $2.5 \times 10^{10}$ & $1.5 \times 10^{8}$ & 3.8 & 6.9 \\
5 & $1.5 \times 10^{10}$ & $2.0 \times 10^{8}$ & 3.3 & 6.9 \\
6 & $5.0 \times 10^{9}$ & $7.6 \times 10^{7}$ & 0.9 & 2.1 \\
7 & $7.6 \times 10^{8}$ & $1.2 \times 10^{7}$ & 0.2 & 0.4 \\
8 & $1.2 \times 10^{8}$ & $4.8 \times 10^{5}$ & 0.02 & 0.03 \\
9 & $2.1 \times 10^{8}$ & $1.0 \times 10^{6}$ & 0.03 & 0.05 \\
\hline
\end{tabular}

has been calculated to be $9 \times 10^{7} \mathrm{n} / \mathrm{cm}^{2}$-s. The average value of this quantity throughout the magnet volume will he approximately a factor of 100 times less than the maximum value. The average value of the total integrated neutron fluence (over all energies) has been calculated to be $3 \times 10^{10} \mathrm{n} / \mathrm{cm}^{2}$-s.

Problem output indicated that 5.25 uncollided $14 \mathrm{MeV}$ neutrons per 100 source neutrons hit the outer walls of the room. An additional 0.67 collided neutrons also hit the walls. Of the 5.25 uncollided neutrons, 4.96 went between the magnets while the remaining 0.29 went through one of the ion sources ( 1.62 uncollided neutrons hit the ion sources; 1.33 of these had reactions with the simulated materials present).

\section{Tritium Generated by Neutron Capture Reactions}

The tritium generation in the nitrogen in the reactor room $\left(9.2 \times 10^{-4} \mathrm{gm} / \mathrm{cm}^{3}\right)$ was calculated to be $3.17 \times 10^{13}$ atoms/s or 48.5 curies/year of full operation. In the borated shield water, the production from the $\mathrm{B}(\mathrm{n}, 2 \alpha) \mathrm{T}$ reaction will be $7.80 \times 10^{13}$ atoms/s or 142 curies/year. If this borated water is recycled over a several-year period, the $\mathrm{B}\left(\mathrm{n}, \alpha / \mathrm{Li}^{7}\right.$ reaction followed by a $\mathrm{Li}^{7}\left(\mathrm{n}, \alpha \mathrm{n}^{\prime}\right) \mathrm{T}$ reaction could produce far larger amounts of tritium. 


\section{SHUTDOWN ACTIVITIES AND DOSE RATES}

With the large neutron fluxes present in the FERF system, there will be large amounts of activation products formed Many of these will be within the system where they will be of interest only when it is necessary to service the machine (e.g., during replacement of the inner wall) while some will be outside in the room itself. The latter will increase difficulty in gaining access to the machine and its associated equipment.

The long-lived activation products formed within the machine were determined from the fluxes calculated during the heating calculation. Table D-9 lists the products in the test region. The Na-24 isotope is listed because it is formed from aluminum; the remainder are formed from impurities existing in aluminum. Table D-10 lists the long-life gamma emitters found in the steel shield. Calculations indi-

Table D-9. Long-life gamma-emitting activation products in test region.

\begin{tabular}{llcc}
\hline & \multicolumn{3}{c}{$\begin{array}{c}\text { Activation } \\
\text { (Ci) }\end{array}$} \\
\cline { 2 - 4 } Isotope & Half life & After 1 year & Equilibrium \\
\hline Na-24 & 14.96 hour & $2.48 \times 10^{6}$ & $2.48 \times 10^{6}$ \\
Pb-203 & 52.1 hour & 0.06 & 0.06 \\
Mo-99 & 66.7 hour & 4.11 & $4.1 !$ \\
Zr-89 & 78.4 lour & 5.41 & 5.41 \\
Sc-48 & 1.83 day & 1.68 & 1.68 \\
Ca-47 & 4.535 day & 0.03 & 0.03 \\
Nb-92M & 10.16 day & 0.11 & 0.11 \\
Nb-95 & 35 day & 0.38 & 0.38 \\
Fe-59 & 45.6 day & 1.70 & 1.71 \\
Sr-89 & 52.7 day & 0.03 & 0.03 \\
Y-91 & 58.8 day & 0.18 & 0.19 \\
Nb-91M & 64 day & 0.06 & 0.07 \\
Zr-95 & 65.5 day & 1.07 & 1.09 \\
Co-58 & 71.3 day & 10.45 & 10.76 \\
Sc-46 & 83.9 day & 0.52 & 0.55 \\
Zn-65 & 245 day & 23.1 & 35.8 \\
Mn-54 & 303 day & 47.8 & 84.3 \\
Na-22 & 2.62 year & 0.07 & 0.32 \\
Co-60 & 5.263 year & 92.7 & 750.8 \\
\hline & & & \\
\hline & & &
\end{tabular}

Table D-10. Long-life, gamma-emitting activation products in shield

\begin{tabular}{llll}
\hline & \multicolumn{3}{c}{$\begin{array}{c}\text { Activation } \\
\text { (Ci) }\end{array}$} \\
\cline { 2 - 4 } Isotope & Half life & After 1 year & Equilibrium \\
\hline Ni-57 & 36 hour & $4.26 \times 10^{4}$ & $4.26 \times 10^{4}$ \\
Fe-59 & 45.6 day & $8.65 \times 10^{4}$ & $8.68 \times 10^{4}$ \\
Co-58 & 71.3 day & $2.62 \times 10^{6}$ & $2.70 \times 10^{6}$ \\
Co-57 & 270 day & $1.33 \times 10^{6}$ & $2.18 \times 10^{6}$ \\
Mn-54 & 303 day & $6.30 \times 10^{5}$ & $1.11 \times 10^{6}$ \\
Co-60 & 5.263 year & $1.51 \times 10^{4}$ & $1.23 \times 10^{5}$ \\
\hline
\end{tabular}

cate that after one year of operation the lead shield contains approximately 80 curies of 52 -hour $\mathrm{Pb}-203$, 2 curies of 245-day $\mathrm{Zn}-65$, and 8 curies of 253-day $\mathrm{Ag}-110 \mathrm{~m}$. The steel container for the magnet contains about 25 curies of 270-day $\mathrm{Co}-57$ and the magnet itself about one rurie of 5.2-yar $\mathrm{Co}-60$.

Dose rates from the listed activation products were calculated only for the 14.96-hour $\mathrm{Na}-24$ produced in the test region. At a point $630 \mathrm{~cm}$ outside the system (point 8 of the previous list), a dose rate of $1.1 \times 10^{4}$ $\mathrm{rem} / \mathrm{h}$ exists immediateiy after shutdown. This point can "see" into the reactor.

Activation of the ion sources and the room walls was calculated as if no additional shielding wete to be placed around the FERF reactor. (In reality, additional components will be present and some shielding will be present.) The reactor was simulated as a large sphere, and the leakage neutrons were simulated as beam sources on titi simulated ion sources and on the two end walls. Figure D-8 shows the location of the reactor and eight detector positions. Point A corresponds to detector 0.8 , which is at the centerine of the reactor. The remaining seven detectors are $1 \mathrm{~m}$ above the floor of the room.

Table D-11 lists the activation products formed and the dose rate from each product at point $A$ immediately after shutdown. A 1-year run is assumed. Figure D-9 indicates the dose rate at shutdown at each of the detector locations. The dose rate at detector no. 5 is larger than the rest because of closeness of this detector to the lower ion source. Figure D-10 indicates similar rates $\mathbf{3 0}$ days after shutdown. In both figures, the dose rate at point $A$ includes an estimate 


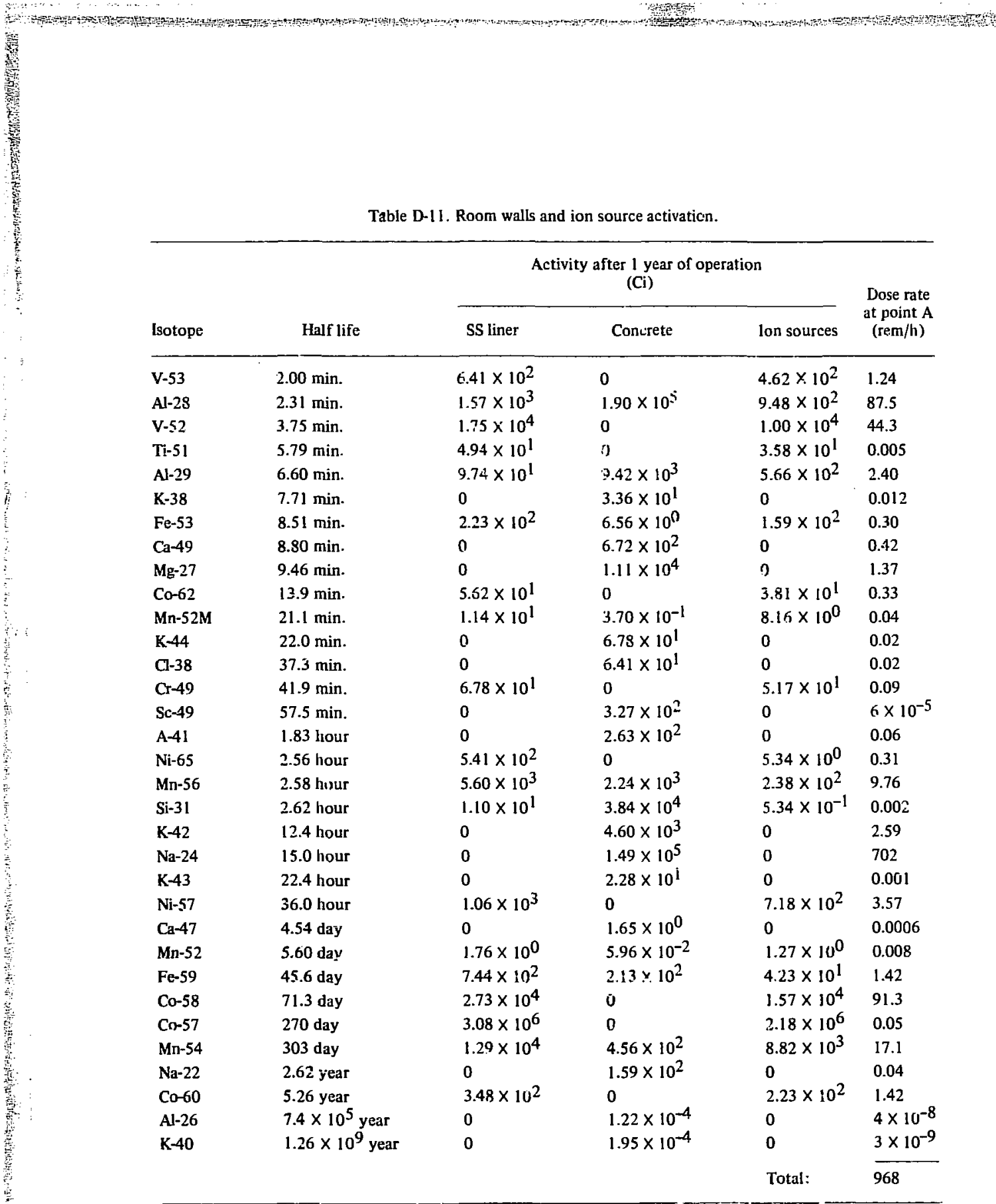




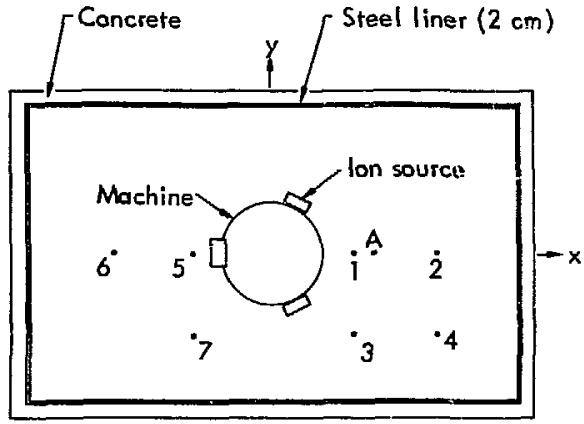

$500 \mathrm{~cm}$

Fig. D-8. Location of detectors within reactor room. Location $A$ is at a z-position at the center of the room and tharefore "sces" into the machine. Locations 1 through 7 are $1 \mathrm{~m}$ above the floor.

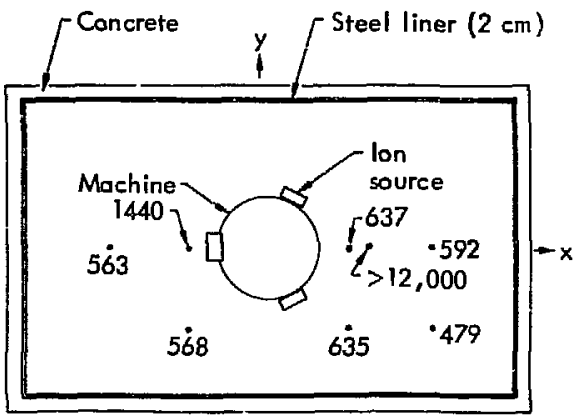

$500 \mathrm{~cm}$

Fig. D-9. D es in rem/h immediately after shutdown fo. .. ang one year of reactor operation.

of the contribution froin inside the reactor. Figure D-1 1 illustrates the time dependence of the dose rate at point one.

\section{CONCLUSIONS}

The shield that has been analyzed in this report is an "ideal" shield; that is, it exists only on paper and does not include all the structural components that will be in a "real" shield. In general, the real shield will not be as good as the one that is analyzed. The drawings for FERF can be used to illustrate this

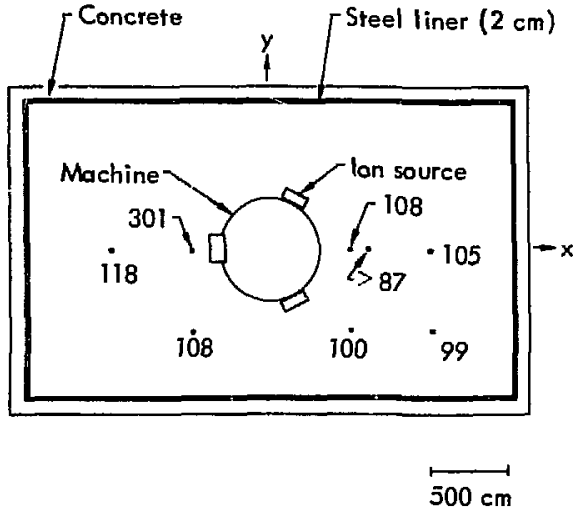

Fig. D-10. Dose rates in rem/h 30 days following shutdown following one year of reactor operation.

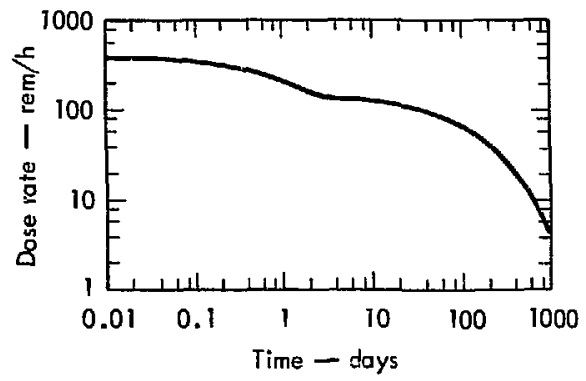

Fig. D-11. Dose rate or time in days at detector position no. 1 after shutdown following one year of reactor operation.

point. For example, the rued to disassemble the reactor for repair or replacement of the first wall has led to a design in which the magnets can be pulled apart. The joint in the shield that is therefore necessary now has a gap through which neutrons may stream directly into the magnet. The heating from these neutrons may be as much as that from the neutrons which must pass through the shield to get to the magnet. It is understood, of course, that this joir: " would never be made as illustrated; other design items .. - adopted which will greatly increase the effective.: , of the shield.

On the other hand, very little work has been done to ma'ke this an optimum shield from the calculational standpoint alone. Many man-years of work and many machine hours of calculation will undoubtedly 
enable the constructicin of a much vetter shield than that described in this report. It therefore seems entirely possible within the existing state-of-the-art to construct a shield for the present design which will hold the heating of the magnet and its case to $5 \mathrm{KW}$.

In arriving at the present design, four different shield designs were calculated. These are illustrated, drawn to the same scale, in Fig. D-12. (The first used a Baseball magnet.) After approximate normalization to the same quantity, and with heating in a steel

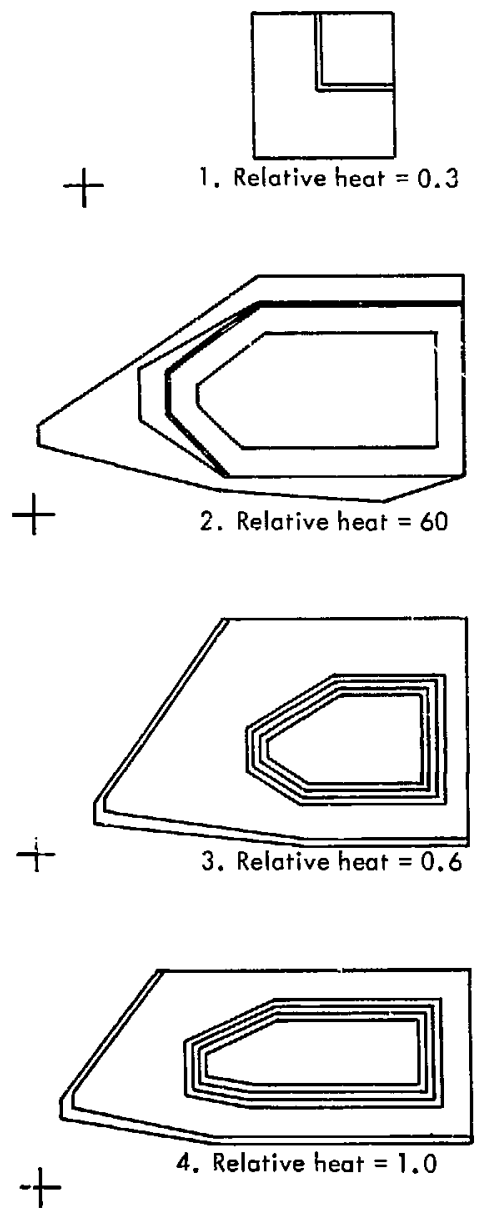

Fig. D-12. Shield designs, showing relative magnet heating (all drawn to sume scale). magnet line: included, the relative heating in the magnet for each design is included on the figure. Only one of the Jesigns has a higher heating than the present design.

The high dose rates in the reactor room after operation may be controlled by appropriate Jesign efforts.

However, the calculated activities will exist somewhere in the systtm, and it is necessary to Jetermine where by making a design judgment as to the philosophy desired in containing the radiation.

\section{REFERENCES}

D1. The MORSE Code-A Multigroup Neutron and Gamma Ray Monte Carlo Transport Code, ORNL Rept. 4585 (1970).

D2. W. W. Engle, $\mathfrak{J}_{r}$, A Users Manual for ANISN. Union Carbide Corporation, Rept. K-1963 (1967).

D3. R. J. Hewerton, et al., An Integrated System for Production of Neut ronics and Photonics Calculational Constants - Volume 4; Evaluated Nuclear Cross Section Library, Lawrence Livermore Laboratory, Rept. UCRL-50400, (1971).

D4. E. F. Plechaty. Photon Cross Sections, $1 \mathrm{KeV}$ to $100 \mathrm{MeV}$, Lawrence Livermore Laboratory, Rept. UCRL-50400, Volume 6, (1968).

DS. C. Wong, et al., Livermore Pulsed Sphere Program: Program Summary through July 1971, Lawrence Livermore Laboratory, Rept. UCRI-S1144, Rev. 1 (1972).

D6. W. E. Alley and R. M. Lessler, Semiempirical Neutron - Induced Reaction Cross Sections, Lawrence Livermore Laboratory, Rept. UCRL-50484, Rev. 1, (1972)

D7. C. M. Lederer, J. M. Hollander, and I. Perlman, Table of Isotopes - Sixth Edition, (John Witey \& Sons, New York, 1967). 


\section{Appendix E - Remote Assembly / Disassembly Procedures*}

\section{OBJECTIVES}

The procedures for remote assembly and disassembly of the FERF reactor assembly and associated equipment were formulated with the following objectives in mind:

- Completely remote operation (i.e., no personnel in the vault);

- All devices to operate in a fail-safe manner;

- Operations to be automated if possible;

- Redundant backup systems to be provided where feasible; and

- Lifting by single-point suspension to be avoided if possible.

\section{GENERAL REQUIREMENTS FOR HANDLING SYSTEMS}

The same equipment can usually be used for both assembly and disassembly. The handling systems fall into two general categories: primarily fixed or rigid systems; and portable or flexible systems with universal application. The fixed systems consist of supporting dollies permanently attached to the large reactor subassemblies which operate on tracks imbedded in the floor of the vault. Subassemblies such as the expander tanks, first- and second-wall assemblies, and half tanks are mounted on dollies which support the entire reactor assembly.

Reactor system components that either cannot be supported by permanent dollies or that cannot be mounted by pure translational movement can be handled by a combination of lifting devices, portable dollies, and special-purpose temporary fixtures. Such components include the superconducting magnet coils, C-clamps for the magnetic coils, and some of the injector clusters and plasma collector plate units. The positioning and clamping of the mating surfaces of individual components prior to pumpdown can be similar for all reactor subassemblies.

*Acknowledgement is given to Miles G. Patrick and Scott Kelley of Battelle-Pacific Northwest Laboratories who assisted in preparation of this Appendix.
The initial convept drawings of the reactor vault indicate a vault approximately 60 feet high and 55 feet wide. A slot or trench down the centerline of the floor of the vault reduces the height of the assembled reactor above the floor by permitting projecting portions of the half tank and one expander tank to extend below the floc: level. The trench separating the major subassemblies permits access to the inner components. However, if it is found that the trench inconveriently limits the distance and direction that the major subassemblies can be moved away, the problem can be avoided by mounting the entire reactor structure above the floor level. Two sets of parallel tracks along each wall of the vault and appropriate switching mechanisms will permit the components from $t$ 'e inner core to be placed on dollies which could then be shunted around the subassemblies that had been moved away. Mounting the entire assembly above the floor will simplify mounting and dismounting the components beneath the reactor structure.

All operations in the assembly and disassembly will be monitored and conducted from a central control room outside the vault. About 10 fixed and portable remotely operated TV monitors equipped with zoom lenses will permit viewing of all areas within the vault. The control room is included within a section of the reactor control room. A console within the control room contains all of the required system operating controls, TV monitoring screens, and instrumentation readouts.

Because the atmosphere within the reactor vault is nitrogen, all equipment and instrumentation within the vault must operate in nitrogen. Personncl entering for any reason must use a self-contained breathing apparatus.

\section{TRANSLATIONAL MOVEMENT USING INTEGRAL DOLLIES}

Dollies will be integral parts of the major reactor subassemblies. Major structural components (e.g., the half tanks, expander tanks, and first- and secor $\mathrm{d}$ wall assemblies) can then be assembled and disassembled by pure translational movement back and forth along a set of tracks. The driving mechanisms for the 
dollies can be a redundant set of electrical induction motors driving one or more wheels of each dolly. The motors can brake automatically when activated by a set of limit switches on each end of the travel. For examplc, during assembly the units can be automarically stopped about 1 fooi from the final position, and the motors can then be manually jogged to achieve final position. Power to the motors can be provided by either suspended or trailing electrical cables mounted on constant-tension reels to take up slack. Alternately, those dollies supporting the major subassemblies which move back and forth in a straight line can obtain power from an energized insulated strip of wire (similar to the overhead wires used for electric cars) mounted between the tracks.

The positioning of the dollies during final assembly must be exact to permit accurate connection of ancillary equipment such as coolant piping and vacuum exhaust ducts. All dollies will therefore need an electrically activated mechanical brake and a set of positioning stops on the tracks (to maintain position during assembly). A backup, optical-alignment system would verify the correct position.

Assembly of the large and heavy components of the inner core (e,g., the magnetic coils and C-clamps) will be greatly facilitated by splitting the half-tank assemblies in a horizontal plane. The bottom half of the half-tank assembly can be locked in final position and the coils, $\mathrm{C}$-clamps, and portions of the first-wall assemily set into the half tank. The extreme weight of the C-clamps can be accommodated during assembly by separating each clamp into at least four sections and fastening the sections together with nuts and bolts. The nuts can be captive on the assembly and installed by remotely operated impaci wrenches suspended from the crane or mounted on remotely controlled dollies.

The configuration of the slot between the superconducting magnet coils precludes withdrawing the first-wall assembly through the assembled coil by sliding it away from the center of the reactor; therefore, a parting seam is required between the coils on the first-wall assembly.

Because of the composite weight of the reactor components, a single set of tracks may not be adequate for support and movernent of the dollies. This can be overcome by nesting the dollies and using two sets of different-gage tracks. Then the dollies supporting the half tanks would ride on both sets of rails, and the dollies under the first- and second-wall assemblies and the expander tanks would be mounted only on the outer tracks.

\section{USE OF AN OVERHEAD CRANE}

A travelling overhead crane with lifting capacity of approximately 60 tons will handle the reactor components during assembly and disassembly, providinis the extremely heavy C-clamps are divided. During disassembly, inner components such as the C-clanps and magnetic coils would be stored on the floor in jigs which provided storage in the correct position for assembiy. Lifting lugs would be permanent where possible, where not, removable pad eyes would be screwed into the units.

A portable machine with two manipulator arms could perform some operations in the assembly/disassembly procedure such as installation or removal of components and ancillary equipment located beneath horizontal projections of the reactor structural subassemblies and therefore not accessible to the hook of the crane. Considerable design effort should be spent on methods to preclude the use of such a machine because, first, it would be very expensive and complex, and second, operation of remotely operated portable manipulators cannot normally be made fail-safe.

Srnaller components and ancillary equipment such as vacuum lines, cooling water lines, sample exposure tubes, plasma collector plate, and injector clusters can be mounted and dismounted on an individual basis using a combination of portable dollies, the overhead crane, and temporary jigs and fixtures. Assembly/ disassembly will be greatly simplified if movement is restristen to a single translational motion or to pivoting around a single axis.

If the support assembly for the injector clusters or. the vertical half tank is split in a horizontal plane, the injector clusters can be simply iswered or raised for assembly onto the top and botium of the vertical half tank. The injector clusters mounting into the horizontal half tank are attached to dollies that ride on tracks parallel to the centerline of the injector cluster unit. Assembly/disassembly then requires only pure translational movement to and from the reactor structure. 


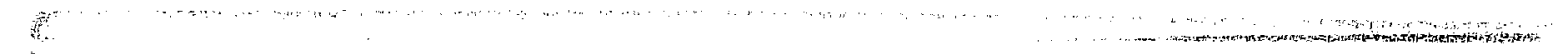

The plasma collector plate units are mounted onto the horizontal expander tank by translational movement. However, the plasi. collector plate units which are mounted on the vertical expander tank cannot readily be assembled or disassembled by translational movement. The lower plasma collector plate unit will require a dolly with an elevatable platform. Elevation would be along an axis perpendicular to the plane of the mating surfaces rather than vertical to the floor. The upper plasma collector plate on the vertical expander tank will be mounted and dismounted using a combination horizontal and vertical movement with the overhead crane.

The sample tubes and piping for the cooling water will be connected and disconnected rising a sliding manifold that simultaneously locks and seals all tubes. The sample tubes will be difficult to seal if cooling is required because the tubes may then become annuli and require a double seal. The manifolds will be moved by electricaliy driven lead screws which are attached either to a rigid structure such as the reactor vault floor or to portions of the reactor structure.

The vacuum exhaust ducts and other attached ancillary hardware will be assembled and disassemblcd either by attaching them to electrically powered pivot arms that swing the ducts out of the way or (possibly) by a ram that moves the ducts back and forth in translational movement. The nanged joints wili be slightly beveled from the horizontal (see Fig. E-i) so that the assembled units will be wedged tightly after assembly.

An alternative assembly/disassembly me:hod for the coolant piping; sample exposure tubes, and exhaust ducts would be to provide a telescoping section. This section would have to retract enough to permit clearance between joints and alsn clearance to move other components past the remaining piping.

\section{RENOTE POSITIONING AND CLAMPING}

The joints formed by mating surfaces of the major reactor subassemblies to smaller system components such as injector clusters and plasma collector plate units require an initial alignment during each step in the assembly followed by a simultaneuus clamping pressure on all joints of this assembled reactor prior to pumpdown. The system cannot be evacuated unless

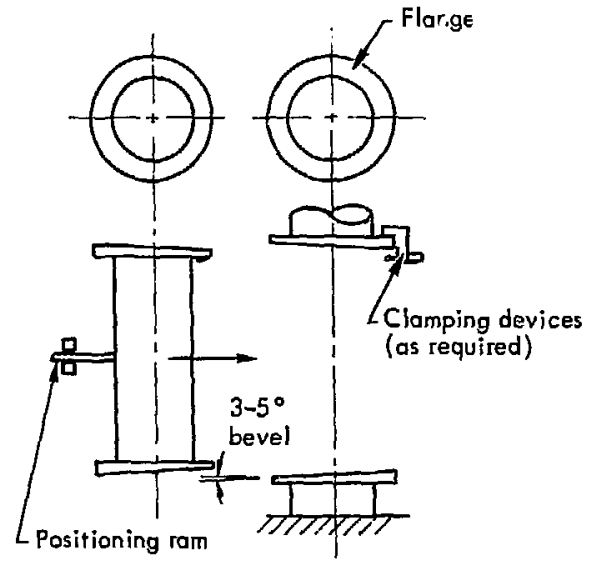

Fig. E-1. Schematic of tapered flanges.

the assembly is complete. Thus, the joints must be clamped together while reactor subassemblies are being assembled or disassembled, particularly if gravity would cause the component would fall oft the reactor when the reactor core is not evacuated.

All mating surfaces forming joints between subassemblies or components are assumed to be planar. It is further assumed that metallic 0 -ring seals will be used at each joint interface and the mating surfaces will, therefore, consist essentially of a pair of flanges. The flanges must be wide enough to permit an adequate radius in the metallic D-ring at all corners of rectangular cross sections. A 3-inch flange width is the minimum acceptable width to provide room for an adequate (4-inch-minimum) radius.

The alignment between all mating surfaces will be established hy a set of tapered guide pins (Fig. E-2) on the flange of the subassembly on the component being moved. The pins will enter taper:d holes on the flange of the subassembly to which the noving component is being fitted. The size and sumber of guide pins required per joint will depend on the weight of the component being moved into position and on any existing prealignment. For example, the joint between half tanks will require only two alignment guide pins on opposing flanges because both subasismblies will be mounted on dollies which serie to prealign the joint, and assembly requires only pure translational movement of one 


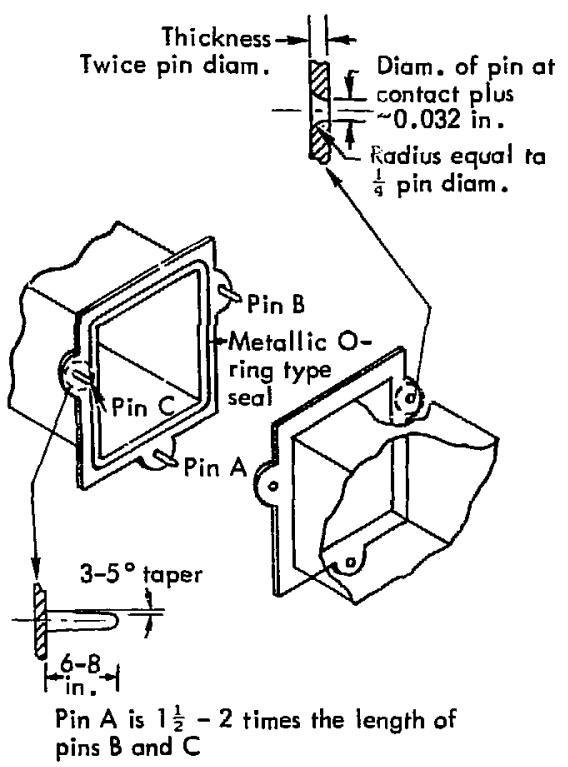

Fig. E-2. Schematic of guide pins.

or both of the units. Positioning of the injector clusters and plasma collector plate units, however, will require three or more guide pins because of little or no prealignment capability and because the mating will require movement in two planes.

To permit initial engagement, one of the guide pins on components moved in two places will be longer than the remaining pins. The assembly will then be jogged to facilitate engagement of the remaining pins. The jogging will be done by suspending each component from a bridle and lifting one of them with the overhead crane. If components require precise axial alignment during assembly, a second set of smaller guide pins and tapered holes could be provided that v. 11 engage after the mating surfaces are almost in contact.

The devices for clamping and holding the joints together are shown in Fig. E-3. The clamping devices will be used on all mating surfaces or joints which have a rectangular cross section. Joints with a circular cross section (such as the pipes from the roughing pumps) will be clamped with a split-ring, wedge-type clamp (Fig. E-4) which is actuated by retating lead screws on the top and bottom. All clamping devices

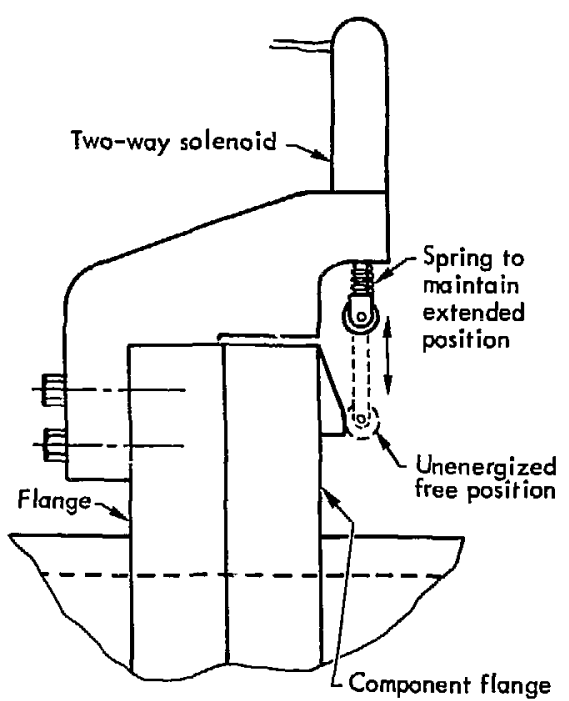

Fig. E-3. Clamping device.

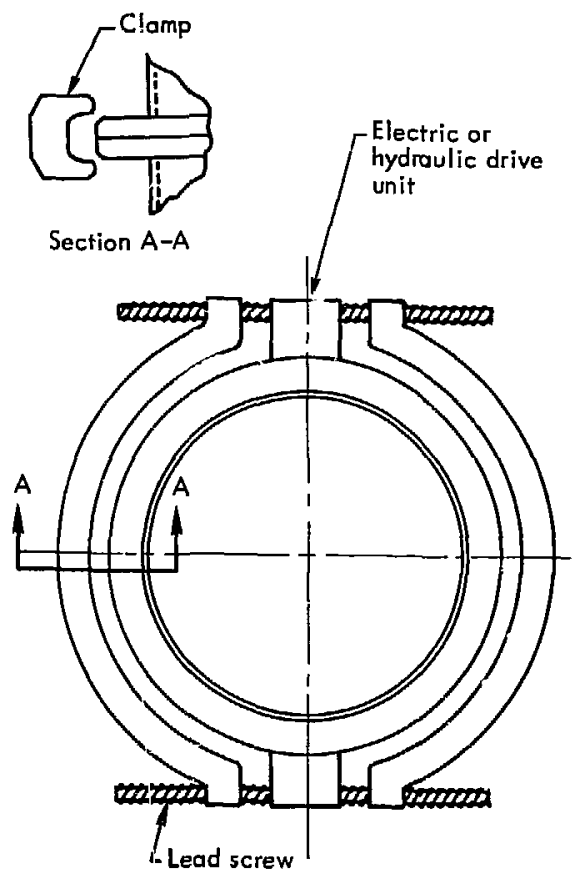

Fig- E-4. Split-ring wedge clamp. 

Appendix F - Safety Analysis

\section{PHILOSOPHY}

A major goal of every facility engineering design effort is to assure that any potential hazards to personnel working in the facility, to personnel around the facility, and to the general public are reduced to the lowest practicable level. To this ind, facility design must provide protection under bott. operational and potential accident conditions so that exposures will be kept well within applicable federal, state, and regional regulations and guidelines.

It is the intent of this section

- To identify the most significant potential safety problem areas associated with FERF, and

- To discuss them in sufficient detail to determine which can be dealt with satisfactorily using normal good safety practices and which will require special design features and terhnology.

\section{SOURCE OF HAZARDS}

The primary safety problems associated with FERF come from the specific operational requirements of a pilot fusion reactor. These may be summarized as follows:

- The use of large quantities of deuteriurn and tritium as the fuel source for the fusion reaction.

- The fast-neutron flux associated with the DT burn. The flux results in high radiation levels during operation ind in the production of large amounts of beta and gamma-emitting activation products.

- The use of large amounts of electrical energy to provide the necessary electromagnetic fields for plasma control.

- The large heat loads that result from the developed electromagnetic fields and the DT burn.

\section{TRITIUM USE}

Inventory

As indicated in Appendix $\mathrm{G}$ of this report, about $400 \mathrm{~g}(66.6 \mathrm{~mol})$ of tritium gas and about $800 \mathrm{~g}$ ( $200 \mathrm{~mol}$ ) of deuterium gas will be employed in the operation of the FERF facility. This represents the maximum amount of material that will be present in the facility at any one time. More sigrificant, however, is the distribution of the material during operations. At the end of each cycle, the total tritium in the four injector modules is estimated to be about $60 \mathrm{~g}$. The cryogenic distillation columns which are part of the isotopic separation process are expected to contain another $270 \mathrm{~g}$. The remaining tritium will be distributed throughout the recycling system, with a portion kept in storage as reserve supply.

Note that during recycling of the tritium inventory, the tritium pressure will be less than ambient and, in fact, the system will generally be under a hard vacuum. Thus, potential leaks will be kept to a minimum.

Another tritiun source is the borated water planned for use in the cooling water for the magnet shield. Calculations indicate that about $140 \mathrm{Ci}$ of tritium as $\mathrm{T}_{2} \mathrm{O}$ will be produced each year as a result of neutrnn interaction with boron-10. Provisions must be made for periodic replacement of this water and its subsequent disposal.

\section{Toxicity}

The toxicity of tritium is rather well known and has been documented in a number of publications. Tritium is considered a moderately toxic radioisotope and has been assigned recommended concentration guides (RCG) for personnel exposure (see Table F-1). If continuously present, these concentrations will result in exposures to an individual of approximately $5 \mathrm{rem} /$ year for the controlled area $(40 \mathrm{~h} /$ week $)$ and $0.5 \mathrm{rem} /$ year for the uncontrolled area $(168 \mathrm{~h} /$ week $)$.

Table F-1. Recommended concentration guides for personnel exposure.

\begin{tabular}{lcc}
\hline Isotope & $\begin{array}{c}\text { Controlled area } \\
(\mu \mathrm{Ci} / \mathrm{m} \ell)\end{array}$ & $\begin{array}{c}\text { Uncontrolled area } \\
(\mu \mathrm{Ci} / \mathrm{m} \ell)\end{array}$ \\
\hline${ }^{3} \mathrm{H}$ (HTO) & $5 \times 10^{-6}$ & $2 \times 10^{-7}$ \\
H( HT) & $2 \times 10^{-3}$ & $4 \times 10^{-5}$ \\
\hline
\end{tabular}

To place these numbers in perspective, however, one must factor in the rather large amounts of tritiun involved in the FERF facility. For example, 
exposure of an individual to a concentration of $10 \mu \mathrm{Ci} / \mathrm{ml}$ of tritium as HTO For a period of 10 minutes will result in a whole-body dose of 480 rem. (If the tritium form were HT the exposure would be 300 mrem.) Assuming static condition in a room such as the reactor vault which is $50 \times 150 \times 60$ feet (about $450,000 \mathrm{ft}^{3}$ ), this concentration would be attained through the release of $15 \mathrm{~g}$ of tritium as HTO.

To achieve the RCG value of $5 \times 10^{-6} \mu \mathrm{Cl} / \mathrm{ml}$ in the same room, again assuming static conditions, a release of only $7.5 \times 10^{-2} \mu \mathrm{Ci}$ or about $7.5 \times 10^{-6} \mathrm{~g}$ of tritium would be required; obviously, in either case, this is a small fraction of the total tritium available. Thus, to achieve the required measure of control. the tritium containment system nust be designed as leakproof as possible. Much of the required basic tritium containment technology exists today; however, where in use, it is on a small scale and mainly at several AEC facilities (including LLL, LASL, and the Savannah River Project).

Special provisions must also be made in the design and operation of FERF to minimize personnel exposures to tritium during disassembly and maintenance operations. This will be especially true for any surfaces that may contain tritium due to permeation and that may outgas during exposure to the air. Special precautions that may be employed include pressurized suits and local close-capture hoods or enclosures. Plastic suits are now available that reduce exposure levels by $10^{5}$.

\section{Environmericiaj Effects}

Accidental release of gram quantities of tritium to the environment is not considered credible recause the proposed design provides for at least double containment of the tritium system. Since the integrity of the containment system is essential to minimum release of tritium, the quality assurance program developed for the project must insure continuous operation of the containment system throughout all postulated design-basis accidents.

It is certain, however, that small amounts of tritium will be released routinely and continuously from the facility due to permeation of the metal wall and transfers of equipment and personnel through air locks. Maximum probable losses for these potential release points are listed in Table F-2.
Table F-2. Maximum probable routine tritium losses.

$\begin{array}{r}\text { Release point } \quad \text { Loss (Ci/year) } \\ \hline\end{array}$

Permeation through metal wall:

20

Three small air locks, assuming 10 trips/day:

900

Two large air locks, assuming

2 tuips/year:

1100

Total: $\quad 2020($ or $\sim 2000)$

If it is assumed that these releases occur at LLL and the appropriate diffusion parameters for average annual meteorological conditions are applied, the total manrem dose to the population within a 50-mile radius would be less than 100 man-rem per year. Since the total population affected is $\mathbf{4 . 6}$ million, the average lose would be about $2 \times 10^{-2} \mathrm{mrem} / \mathrm{man}$-year.

The potential impact of an accidental release of tritium was assessed by assuming loss of $1000 \mathrm{Ci}$ of tritium as HTO over a 24-hour period. The dose due to inhalation skin absorption to an indjvidual at the site boundary was found to be less than $0.3 \mathrm{mrem}$, well within the guidance of Reference Fl. This excludes the possibility of local rainout.

\section{RADIATION LEVELS}

\section{During Operation}

During normal operation of the facility, the DT reaction in the plasma is expected to yield about $3 \times 10^{18} \mathrm{n} / \mathrm{s}$. This will require the use of rather large amounts of shielding to provide not only personnel protection but also radiation-damage protection for the magnetic and other critical parts of the fusion reactor. In the current design, the magnet shield consists of steel with borated water for cooling: however, engineering and physics requirements may call for other materials. Concrete is used for the biological shield. Radiation levels during operation of FERF will be reduced to acceptable levels through the appropriate use of shielding. The technology of calculating and employing suitable shielding is well understood. 


\section{After Shutdown}

The primary radiation problems associated with FERF will occur as a result of neutron activation of materials used for shielding and containment. Activation of other parts of the system (such as corrosion products in the coolant water) was considered and found to be insignificant. Tritium produced in the borated water will not add to the external radiation levels.

Probable radiation levels in the area surrounding the plasma containment vessel following shutdown of the plasma were calculated assuming the materials of construction include stainless steel and other metals commonly used for containment. The dose rate immediately following shutdow'n was found to be approximately $1000 \mathrm{R} / \mathrm{h}$ at $600 \mathrm{~cm}$ from the center of the reactor vessel.

Any maintenance operations or modifications that must be performed on the magnet and the containment vessel will require the use of extensive personnel shielding and/or remote hand ling equipment. Allowing time for cooling following shutdown will help little due to the long half lives of the activation products. The technology developed at ridors, fuel processing plants, and isotope laboratories can be applied directly.

\section{ACCIDENT ANALYSIS}

Accidents affecting the facility operation may be divided into two categories: those caused by acts of nature and peculiar to the site location (e.g., earthquakes, tornadoes, floods, lightring), and those usually caused by the human element (e.g., operational error or equipment failure due to improper design or maintenance). The facility must be designed so that, if any one of the accidents considered credible for the facility should occur, those features and systems essentiai to safe shutdown will continue to function and nu loss of containment will occur.

The principal accidents that have been considered in the design of this facility are discussed briefly below.

\section{Collapse of the Vacuum Vessel}

Collapse of the inner vacuum vessel that contains the plasma is considered highly improbable because the void surrounding the vessel is maintained at a pressure only slightly above the $10^{-6}$ Torr. Even if lirst-wall failure should occur, a second shell thick enough to contain the pressure vessel surrounds the inner vessel.

\section{First-Wail Failure}

The first wall is cooled by high-pressure, fast-flowing cold water carried in thin-walled tubes. These firstwall tubes will receive massive neutron doses and high heat loads. They must withstand severe sputtering, several kinds of radiation damage, embrittlement, and thermal stresses. A tube might fail, although this seems highly unlikely because two layers of essentially parallel coolant lines are provided. As a consequence, the plasma would immediately go out and the plasma vacuum chamber would partially fill with water. The water in the coolant loop would therefore become tritrium-contani nsed. The coolant water will not run directly to a cooling tower but will first go through a water-water heat exchanger which would be located within the biological tritium shield. Thus, should the coolant water become contaminated, it can be removed and disposed of safely.

The same considerations apply to the water that would leak into the main chamber. The volume of witter would be less, but its tritium content would be higher. This water might be orained from the "issel, punped directly onto molecular sieves, or evaporated and trapped by the main tritium recovery system.

Any local cleanup would be extremely difficult due to the high radiation levels caused by induces radioactivity. Special suits designed to reduce tritium exposure would be used.

\section{Chemical Explosion of the Fuel}

Deuterium and tritium are used in sufficient quantitites to constitute an explosion hazard should the gases leak and become mixed with air. This potential problem has been eliminated by repla.ing the atmosphere surrounding all deuterium-tritium bearing lines and vessels with an inert gas such as nitrogen.

\section{Magnet Shield Failure}

Present plans call for borated water to be used in 
the magnet shield to provide neutron shielding and cooling. As indicated earlier, the water will become contaminated with tritium due to neutron interaction with the boron. Any leakage of this liquid will result in the same problems indicated for first-wall failure.

\section{Vacuum System Failure Outside the Vacuum Vessel}

Vacuum system failure outside the vacuum vessel would result in the leakage si nitrogen into the vacuum lines. Some tritium would leak uut into the main room, but this could be cleaned up with the main deuterium-tritium recovery system. Line repair and cleanup would again require the use of protective suits.

\section{Tritium Distillation System Failure}

The distillation columns are the only components of the entire fuel-handling system that run above atmospheric pressure. They receiye no neutron radiation damage, and the chance of their failing is extremely remote. There is a possibility of valve failure, but this too is considered remote due to the quality assurance program that will be applied to this system.

The still and other cryogenic components will be protected by a vacuum jacket and therefore are also doubly contained. Loss of vacuum in the jacket could result from nitrogen leaking in from the outside or from DT leaking in from the column. Although loss of vacuum in the jackst will automatically transfer operation to the other still, it will not neressarily esult in a tritium release. Nitrogen leaking in from the outside would be of little consequence other than requiring some repair work. It would result in rapid boiling of the entire still inventory. A pressure surge could result which would either be contained by the inherent strength of the system or, more likely, be relieved by rupturing an all-welded rupture disk which would abort the system to a large reservoir. Filling the vacuum jacket with DT would cause loss of vacuum with the sar.e consequences. The equipment would require disassembly for repair, but it could probably us drane with relative ease. If tritium did escape, it would be into the nitrogen atmosphere being processed ty the main recovary sysiem. Potential problems with the tritium distillation system can be controlled using existing technology.

\section{Magnet Failure}

Super-conducting magnets usually operate during charging and discharging at very low yoltages, in the order of 2-5 V. However, should the magnet lose its superconductivity due to rise in tenperature or other problems, it has been designed to carry the load sa' ely. Since loss of superconductivity could cause conversion of the liquid helium to the gaseous state, the helium lines must be protected with overpressure relief valves. The basic design of superconducting magnets, plus special safety features included in the design of the 5ERF magnet, eliminate melt-down as a credible accident.

\section{APPLICABLE REGULATIONS}

\section{Safety Analysis}

The design and construction of a facility such as FERF is subject to critical seview by a variety of state and federal agencies. A key point, yet to be settled, is whether FERF is to be treated on the same basis as a fission reactor. Based on recent AEC policy with respect to reactor fuel reprocessing and plutonium handling facilities, it seems likely that most of the regulations written for power reactors will be applied to FERF. Given that this is true, the principal regulations to whick FERF will be subject are contained in Title 10 of the Code of Federal Regulations $F 1$, especially Part 20 -- "Standards for protection against radiation," Part 50 - "Licensing of production and utilization facilities," and Part 100 - "Reactor site criteria," plus appropriate appendices. If the project is developed by an AEC contrazior, then the desigr. and operation become subject to the various $\mathrm{AEC}$ Manual chapters.

AEC Manual, Appendix 6101, Annex $C_{\text {, }}$ dated April 8, $1971,{ }^{F 2}$ requires the preparation of both preliminary and final safety analysis reports as part of the design of any major facility that has the potential for significant environmental impact. Contents of the documents may vary somewhat depending on the specific characteristics of the proposed facility, but in general they must follow the format recommended in "Standard Format and Content of Safety Analysis Reports for Nuclear Power Plants" puilished by the Regulatory Staff in October 1972. Requirements for safety analysis reports are also indicatea in Section 
50.34 of Reference $\mathrm{F} 1$.

Principal features of safety analysis reports incluce a detuiled description of the proposed site, a presentation and discussion of "Jesign basis accidents," an identification of those structures, systems, and components essential to the safe operation of the facility, and a detailed presentation of the measures that will be taken to assure that those systems will continue to function properly through the postulated design basis accidents.

\section{ENVIRONMENTAL ASSESSMENT}

AEC IAD 05 10-29 $F 3$ requires environmental assessments for all proposed line itemıs, major general plant projects, major equipment, and other major activities. The assessment must include the following sections:

- Description of the proposed action and its anticipated benefits.

- Potential environmental impact.

- Alternatives and their environmental impact.

- Cumulative and long-term effects of the proposed action.

- Irreversible and irretrievable ccmmitments of resouices.

- Potential conflict with federal, state, or local governments.

- Cost-benefit analysis.

On the basis of this seven-point document, the AEC, Division of Operational Safety, determines the possible need for an environmental impact statement as required by the Nationai Environmenta Policy Act of 1969. It seems likely that such : document must be prepared for this facility and reviewed prior to construction.

\section{CONCLUSIONS}

The FERF described in this report will be designed to ensure the safety of operating personnel a: 1 the general public at all times during nermal operations and during possible accident conditions. The several possible accidents briefly discussed in this Appendix represent those considered to be of greatest concern in the design and operation of the facility. As indicated, the most significant safety challenges will be presented by problems associated with containment of the tritium and the high radiation levels due to neutron activation. Both of these areas, however, can $1: x$ surcessfully treated with the current level of technology employed by several of the AEC contractors.

\section{REFERENCES}

Fl. Code of Federal Regulations, Title 10, Atomic Energy, GSA (Rev. January 1, 1973).

F2. AEC Manual, Appendix 6101, Annex C, AEC (April 8, 1971).

F3. AEC Manual, Immediate Action Directive, AEC, IAD No. 05 10-32 (November 13, 1973). 


\section{INTRODUCTION}

The FERF would be the firs! high-power, continuously operating reactor in the world. Since tritium will be used, a major class of tritium handling and safety problems mist be solved.

Most of the topics which will be discussed in this Appendix fall logically intu one of two categories: fuel recycling and fuel continement. The former includes all the operations and processes which must be performed to maintain the fueling of FERF in a reliable, steady-state condition for periods of several months. The latter, confinement, includes all the measures necessary to assure that no one inside or outside the facility will ever, even in an accident siluation, be exposed to raviation ciosages of unacceptablv high levels.

\section{FUEL CYCLE}

\section{General}

A very schematic diagram of the fueling system is shown in Fig. G-1. Note that almost the entire gashandling system will be operating below atmospheric gressure. In fact, much of the equipment will ruis under conditions usually described as hard vacuum. Theretore, there is little reason to believe that a leak anywhere in the system would automatically cause a major release of tritium. This will be true even though there is a relatively large tritium inventory $(400 \mathrm{~g})$ in the facility. (Leakage in air poses a separate set of problems.)

\section{Vacuum Pumping the FERF Reactor}

The FERF reactor is pumped in two principal regions: in the injector modules and in the tank receiving the mirror end losses. Because of the high gas loads and space limit ations, the most demanding requirements are found in the injector region. From the standpoint of minimizing the tritium inventory, we would prefer to use mercury diffusion pumps; however, because of the spatial restraints, cryopumps must be used in the injector modules. At any given moment, three of the injector modules will be operat-

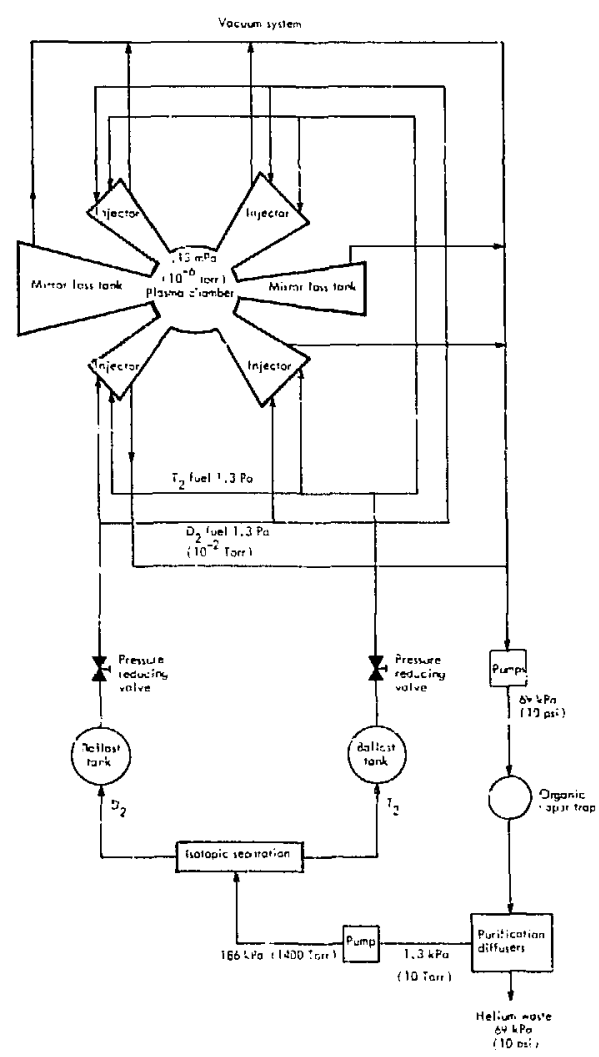

Fig. G-1. Fuel system flow' ciagrani.

ing and one will be undergoing regeneration of its cryopump. The planned cycle time for each injector module with its respective pump is about 30 minutes. Under these operating conditions, the injectorcryopump modules will have a combined tritium inventory of about $60 \mathrm{~g}$ at the end of each cycle.

The possibility of using one injector module for tritium and the other three for deuterium was considered. (To optimize plasma fueling, the deuterium and tritium are injected at different energies.) If in each injector module one could use an array of small cryopumps which would be sequentially isolated from the machine and regenerated, then the total tritium inventory rould drop by a factor of three. 
Only part of this inventory reduction would result from a reduced quantity : $n$ the cryopumps; the rest would come about because a smaller inventory could be maintained in the isotopic separation plant. By keeping the two isotopes separate during pumping operations, the unused gas could be directly recycled to the injectors without first passing through the isotopic separation equipment. However, as with the diffusion pumps this option became unavailable because of the demands for unusually high pumping speed. A large number of smaller pumps could not be accommodated in the space ava ilable.

In the region where the mirror end losses are removed, diffusion pumps might be used: the choice $w_{1}$ ll be on the basis of convenience rather than tritium inventory. Because the gas loads are so much lower in this region, the tritium inventory associated with cryopumps can be small compared to that in the injector region.

\section{Palladium Diffusers}

Several impurities may accumulate in the fuel. The most obvious one is helium, which will be produced by iritium radioactive decay $\left(\sim 2 \times 10^{-7} \mathrm{~g} \cdot \mathrm{mol} / \mathrm{s}\right)$ and by DT burn in the plasma $\left(\sim 2 \times 10^{-6} \mathrm{~g} \cdot \mathrm{mols} / \mathrm{s}\right)$. In addition to helium, during startup there will be some residual air, small vacuum leaks in the system will admit air (or nitrogen), and surfaces will give off water both by normal outgassing and by hydrogen reduction of oxide films. However, simple calculations show that the bulk of the impurity should be helium.

All impurities must be removed from the fuel. Helium must be removed to minimize blistering of metal surfaces which would otherwise be caused by implantation of energetic alpha particles. Oxygen and nitrogen, if allowed to accumulate, would eventually freeze out and cause plugging in the cryogenic distillation apparetus. Also, frozen oxygen in liquid DT could be hazardous. Therefore, all the fuel leaving the FERF reactor for recycling is permeated through hot $\left(400^{\circ} \mathrm{C}\right)$ palladium-silver membranes before going to the distillation columns.

The physical arrangement for removing impurities from the fuel has already been developed here, both experimentally and theoretically. GI Computer codes exist which accurately predict observed performance.
The FERF purifier uses seven 6.35 -mm-diameter $\times 0.127-\mathrm{mm}$-wali palladium-silver alloy tubes $i$, parallel hookup. The impure gas at $69 \mathrm{kPa}$ (520 Torr) flows on the inside of the tube; a reduced pressure, $1.32 \mathrm{kPa}$ ( 10 Torr), is maintained on the outside so that permeation can occur. After flowing down $7.62 \mathrm{~m}$ of tubing, 90\% of the DT has been permeated. The remainder of the gas inside the tube is combined by manifolding and passes through another $7.62-\mathrm{m} \cdot \mathrm{long}, 6.35-\mathrm{mm}-$ diameter tube with the same operating conditions. The gas is then routed through a final 7.62-m-long tube which is only $1.27 \mathrm{~mm}$ in diameter, but here the pressure outside the tube is held at $0.132 \mathrm{~Pa}(1 \mu \mathrm{m})$ rather than at $1.32 \mathrm{kPa}$. (The pumping capacity required at $0.132 \mathrm{~Pa}$ is only $1 \mathrm{l} / \mathrm{s}$.) The gas exiting from the end of this final tube is $99.9998 \%$ helium and other nonpermeable gases. ${ }^{G 2}$ The helium can be collected and disposed of as low-level radioactive waste. Redundancy is provided so that if a single, small component should fail the entire facility needn't be shut down. A schematic diagram of the separation system is shown in Fig. G-2, with the calculated flow rates which correspond to a nominal injector gas flow equivalent to $3000 \mathrm{~A}$ of diatomic molecules and a nominal fusion power of $5 \mathrm{MW}$. The final reactor design has a fusion power of $3.38 \mathrm{MW}$.

Isotopic Separation of Deuterium and Tritium

After leaving the palladium diffusers, the purified fuel is compressed to $186 \mathrm{kPa}$ (1400 Torr) and sent to the cryogenic distillation columns. This compression may be done by cryopumping so that no impurities are reintroduced to the fuel. One might also consider using a gullium-filled Sprengle pump which would reduce the tritium inventory and, because of gallium's very low vapor pressure, maintain clean gas.

During the initial design study for distillation equipment, we thought isotopic separation could he maintained for the gas being pumped in the injector region. Therefore, the design was based upon a gas load equal to that which entered the plasma region either by injection or streaming. The gas entering the column was expected to consist of the equilibrium composition at high temperature, namely, $0.64 \mathrm{D}_{2}$, and $0.04 \mathrm{~T}_{2}$. The actuai gas load is 12 times larger than that, and the 


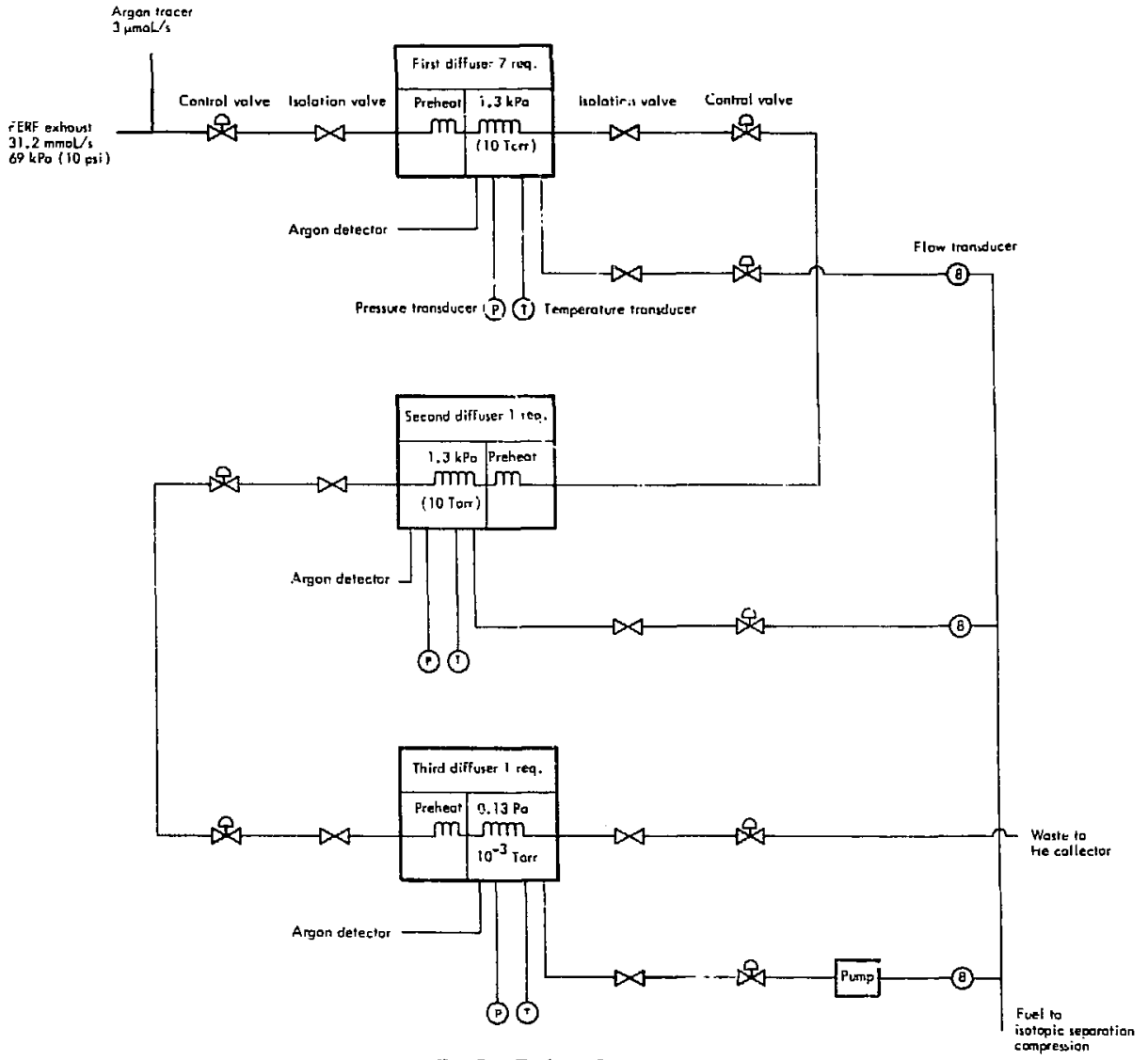

Fig. G-2. Fuel purification system.

expected composition of the gas also has changed slightly.

The original design was bused on a rather complete parametric study of the multistage separation process in a packed column, with a goal of minimizing the tritjum inventory. The required distillate and raffinate streams must be at least $90 \%$ isotopically pure for proper functioning of the fuel injector system. For the reasos; given above, the principal results of the original design study were scaled by a factor of 12 and are given in Fig. G-3. G3 The column was 25.4-mm in diameter and the original tritium inventory was cstimated to be $18 \mathrm{~g}$. Refrigeration requirements for the upper column were $40 \mathrm{~W}$ at $25 \mathrm{~K}$. After scaling to the new requirements, these values become $270 \mathrm{~g}$ tritium and $500 \mathrm{~W}$. It is possible that an injection system can be designed for combined D and $T$ operation, thus greatly reducing the size of the colununs and the tritium inventory. The new column has a 90-rim intemal diameter and is $1.5-\mathrm{m}$ tall. Note that a catalytic isotope equilibrator is used on part of the feed to the lower column. This is nocessary because the deuterium and tritium present as DT molecules cannot be separated by distillation alone. Two identical distillation systems will be supplied to provide redundant capacity in the event of equipment malfunction.

Immediately after isotopic separation, the fuel will be returned to the injectors before significant helium-3 can accumulate by radioactive decay. 


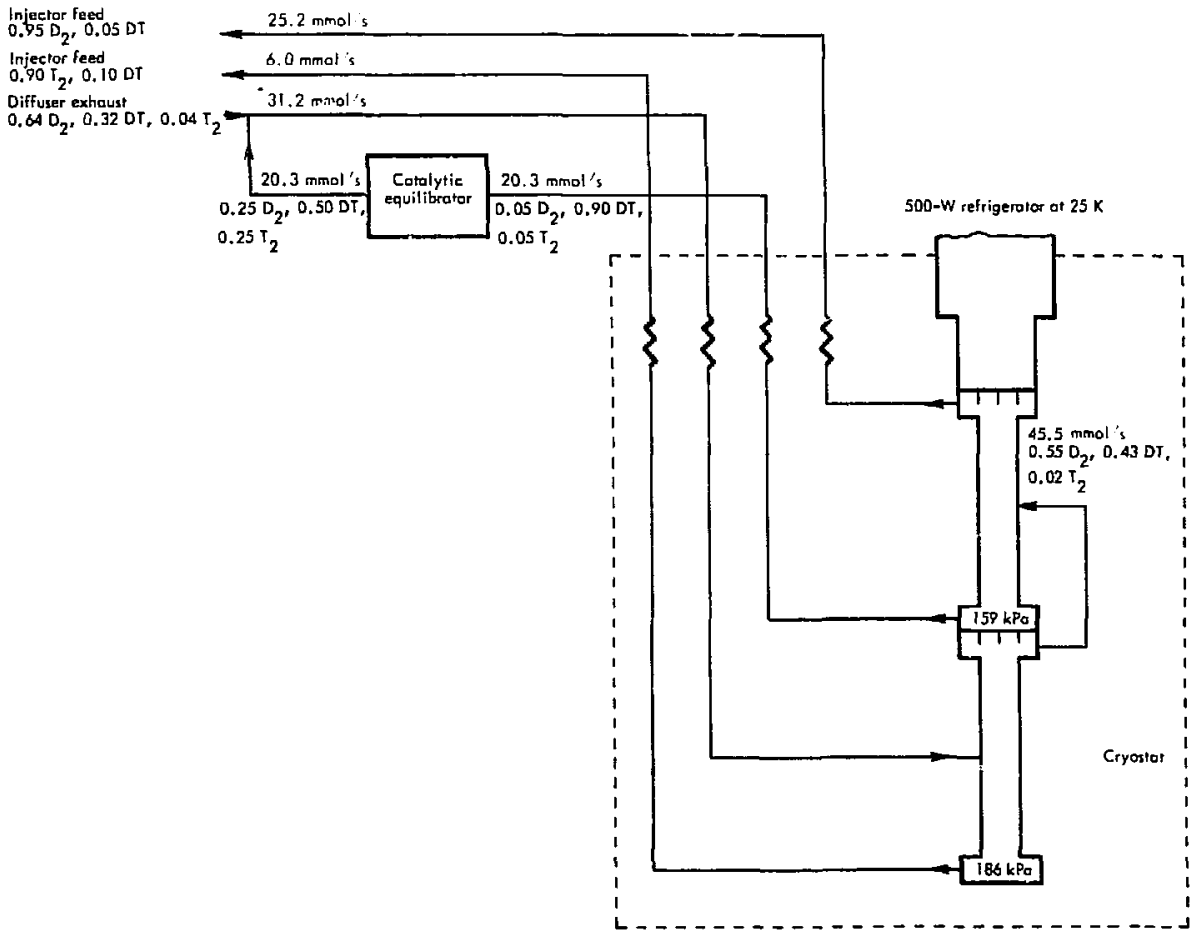

Fig. G-3. Fuel isotopic separation system.

\section{Fuel Storage}

Although not necessarily a part of the fuel system proper, it is clear that provision must be made for safe fuel storage. There should also be a reserve supply to act as a ballast in the event of transient perturbation in the fuel-handling systen. Storage capacity will be provided for the entire facility inventory so that all radioactive gases can be held in a safe configuration during periods of maintenance. If we assume a $400-\mathrm{g}$ (66.6-mol) inventory of tritium, and three times that many moles of deuterium, the storage volume needed is about 45002 at standard conditions. This will be easily provided by tanks isolated from the rest of the facility in a sealed vault so that a large accidental release would not be possible.

\section{TRITIUM IN MAGNET SHIELDS}

Shielding requirements to protect the magnets from radiation are covered in Appendix D. It appears highly desirable to include within the shields a substantial mass of boron because of its high neutron capture cross section and be.:ause no gamma rays are produced. However, although the shields are highly effective in capturing neutrons, they do so in part by reactions which produce tritium. These reactions are

$$
\begin{aligned}
& 10 \mathrm{~B}+\mathrm{n} \rightarrow 2{ }^{4} \mathrm{He}+\mathrm{T} \text {, and } \\
& 10_{\mathrm{B}}+\mathrm{n} \rightarrow{ }^{4} \mathrm{He}+{ }^{7} \mathrm{Li} \\
& { }^{7} \mathrm{Li}+\mathrm{n} \rightarrow{ }^{4} \mathrm{He}+\mathrm{T}+\mathrm{n}^{\prime} .
\end{aligned}
$$


The boron may be present zither as an aqueous solution of boric acid or as a boron-rich solid material. To date, neutron calculations of shie: $i$ performance have been based on aqueous solutions. In what follows, we assume a solution will be used and that it will also function as a heat transfer fluid to remove the heat produced ( $4 \mathrm{MW}$ ) by the neutrons.

For $5 \mathrm{MW}$ of fusion power, it is estimated from calculations that $142 \mathrm{Ci} / \mathrm{yr}$ will be produced in the borated water. $\mathrm{G} 4$ This relatively low level of tritium production can be maintained by regularly removing the lithium produced in the second reaction listed above. Lithium is removed by regular replacement of the solution with fresh. It must be borne in mind that tritiated water is several hundred times more toxic radiologically than tritium gas. ${ }^{\text {G5 }}$ Provision will have to be made for changing the water and disposing of the contaminated solution.

By withdrawing contaminated solt'tion during regularly scheduled maintenance periods and replacing it with fresh solution, the activity level can be kept to levels that are well within the range where present technology and work practices are sufficient to cope with them. A nearly identical situition prevails in heavy-water-moderated nuclear reactors such as the NRX and NRU research reactors at the Chalk River Nuclear Laboratories where the steady-state tritium activity runs from 10 to $50 \mathrm{Ci} / \mathrm{Q}$. 6 With properly trained personnel, good equipment, and well-planned procedures, these operations can be performed safely. The waste liquid can be disposed of either by injecting it into deep wclls or by storing it for a long time under surveillance. G7,G8

\section{CONTAINMENT OF TRITIUM}

\section{General}

Tritium containment is difficult. We must protect the people working within the facility at all times, and we must be able to guarantee with a high degree of certainty that people off-site will never be exposed to nore radiation than is acceptable. The standards to be applied to FERF are not known, but it seems reasonable to assume that present licensing requirements for commercial power reactors might be appropriate. No one living on the site boundary would then be permitted a total additional radiation dose exceeding 5 mrem per year.

Tritium can escape either into the air or into the cooling water, but the former release mode is the more difficult to control. In all present tritium-handling facilities in the United States, the conventional practice is to supply large quantities of fresh-air ventilation on a once-through basis, exhausting the effluent air out a tall stack. In an accident situation, however, the entire spill is released to the atmosphere within a few minutes.

\section{Off-Site Dose Calculations}

Before deciding how to assure environmental acceptability, we must examine the possible consequences of various tritium releases. This has been done using advanced numerical modeling techniques. The interaction of air contaminated with tritiated water exhausting from a $30-\mathrm{m}$ stack with no effective plumie rise was examined for several atmospheric conditions. The site chosen was our own laboratory, which is situated near the eastern end of a small inland valley. Sheep and cattle graze on hills within $1 \mathrm{~km}$ of the eastern boundary of the site.

One case treated postulated the release of $10 \mathrm{~g}$ of tritium as tritiated water during $900 \mathrm{~s}$ ( 15 minutes). This represents a release slightly smaller than one which occurred accidentally in Livermore several years ago, which with once-through ventilation is postulated as a credible accident. $G 9$ The other prohlem treated postulates the release of $0.1 \mathrm{~g}$ of HTO during $86.4 \mathrm{ks}$ (24-h) period which we believe might simulate conditions during major facility maintenance operations. The most pessimistic conditions were found during the dry season $\mathrm{GlO}$

The res'its of calculations based on the 0.1-g release during average dry-weather conditions are given in Fig. G-4. We see that there is a 95\% probability that no one would receive doses greater than 1.0 mrem. However, these same contours can be scaled by a factor of 300 to represent the $10 \mathrm{~g}$ release, where time, quantity, and time-averaged wind direction are taken into account. Clearly, if we impose a 5-mrem maximum acceptable dose incroment, this latter situation would represent an unacceptably high risk.

Two pathways into the body were considered: the direct inhalation/skin route and the vegetation-to- 


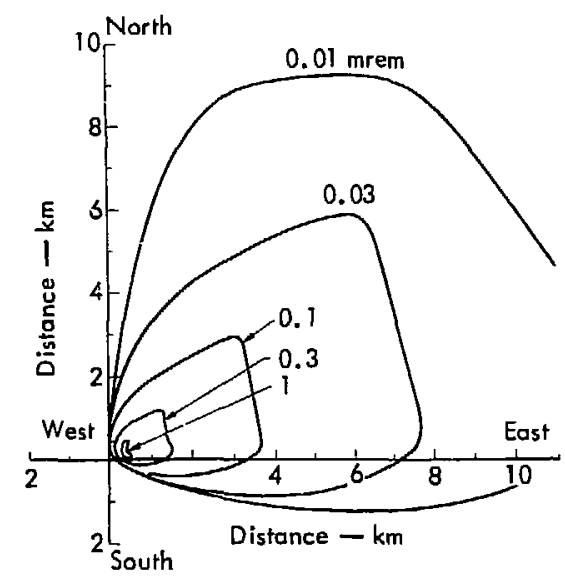

Fig. G4. Dry-season inhalation-skin absorption dose (mrem); $-5 \%$ probability of being exceeded. Calculations assume release of $0.1 \mathrm{~g}$ of HTO in $24 \mathrm{~h}$.

meat-to-man route. The vegetation-to-meat-to-man sequence gave much higher dose estimates, and it would have been necessary to monitor the tritium content of the farm animals after either type of release, regardless of weather conditions. Localized rain during the wet season could give higher tritium. concentrations in some areas.

The general philosophy followed in this study, therefore, has been to follow a total-containment approach in which very little tritium can escape to the outside world.

\section{Permeation by Tritium}

\section{Hot Equipment Walls}

Like other hydrogen isotopes, tritium permeates all metals to a certain exten: Usually, permeation is of little concern except when the equipment operates at higl temperature. In FERF, we would expect hightemperature components in only a few places: the injector sourcis, which are surrounded $b, x$ a vacuuri; the palladium diffusers, which will be st:ondarily contained; and some diffusion pumps, which also can be secondarily contained. (Secondary containment systems will be described later.) Thus, the usually encountered type of permeation can be dealt with in a straightforward fashion.

\section{Cooled First Wall}

In the FERF reactor, there will be an additional and unusual type of permeation, i.e., permeation of implanted high-energy particles rosulting from stray losses in the injected beam, from ile uncaptured beam, from particles scattered out of the plasma, and from mirror end losses. When these particles are imbedded in the metal walls, they will tend to diffuse away from their stopping position toward the vacuum and coolant surfaces of the walls.

The tendency of the implanted ions to diffuse in both directions is independent of the material or its temperature. At steady state, the fraction of tritium diffusing out the coolant surface to that out the vacuum surface equals the atio of implantation depth to wall thickness. This, in turn, is the concentrationgradient ratio shown in Fig. G-5. This simple argument assumes that we do not encounter blistering or peeling of the first wall and that the diffusion coefficient is independent of position.

The estimated total energetic tritium current to all the walls is $200 \mathrm{~A}$, oi $6 \times 10^{-3} \mathrm{~g} / \mathrm{s}$. If the implantation depth is $1 \mu \mathrm{m}$, the tritium permeating to the coolant would be $1.2 \times 10^{-5} \mathrm{~g} / \mathrm{s}$, or more than $380 \mathrm{~g}$ each operating year. This would be unacceptable from at least two points of view: tritium is costly, and contaminated coolant would be an additional hazard.

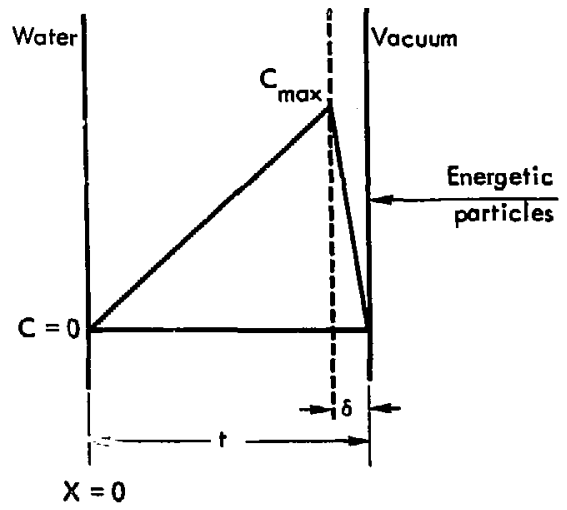

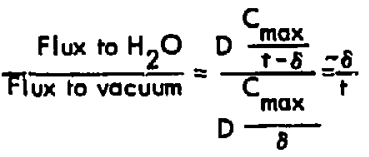

Fig. G-5. Permeation of single-material first wall. 
A composite rnetai wall should take care of this problem. For the metal exposed to the yacuum, we would choose a fast permeator such as palladium or LaNis alloy. It would sover the outer surface of the coolant tubes, in turn made of a slow permeator such as aluminum. The amount of tritium escaping to the coolant would then be strongly material-dependent.

In a composite-tube wall such as this, the isothernal concentration profile of tritium would be something like that shown in Fig. G-6. The solubility and diffusivity of hydrogen is much higher in palladium than in aluminum. The bulk of the palladium is therefort loaded to the tritium concentration, $C_{\max }$, which is determined by the particle flux and diffusion coefficient of tritium in palladium. To calculate $C_{\max }$, we assume $70 \mathrm{~W} / \mathrm{mm}^{2}$ heat load at $90 \mathrm{keV}$ consisting of deuterium and tritium. This corresponds to 1.07 $\times 10^{-7} \mathrm{~mol} / \mathrm{cm}^{2} \mathrm{~s}$. Therl, if the implantation depth, $\delta$, is $1 \mathrm{~mm}$, and the diffusion coefficient is $5.79 \times 10^{-6}$ $\mathrm{cm}^{2} \mathrm{~s}, \mathrm{G} 1 \mathrm{I}$

$$
\begin{aligned}
C_{\max } & \approx(\text { flux })(\delta) / \mathrm{D}=\frac{1.07 \times 10^{-7}\left(10^{-4}\right)}{5.79 \times 10^{-6}} \\
& =1.84 \times 10^{-6} \mathrm{~mol} / \mathrm{cm}^{3}
\end{aligned}
$$

This corresponds to $1.63 \times 10^{-5} \mathrm{H} / \mathrm{Pd}$ atom ratio. For hydrogen in alpha-phase palladium, $\mathrm{Gl} 2$

$$
\log \mathrm{P}_{\text {Torr }}^{\mathrm{i} / 2}=\log \left(\frac{\mathrm{H}}{\mathrm{Pd}}\right)+4.2-\frac{522}{\mathrm{~T}}-\frac{926\left(\frac{\mathrm{H}}{\mathrm{Pd}}\right)}{\mathrm{T}-215} \text {. }
$$

so that

$$
p_{\mathrm{atm}}^{1 / 2}=3.02 \times 10^{-4}
$$

In these calculations, it was assumed that the wall temperature was $350 \mathrm{~K}$. The actual temperature of the palladium will drop as the palladium erodes because its thermal conductivity is so much below that of aluminum.

Knowing the equivalent equilibrium pressure of hydrogen in the palladium, we invoke thermodynamic equilibrium across the Al-Pd boundary. This is what gives us such a large drop in tritium content even through the chemical activity (effective partial pres-

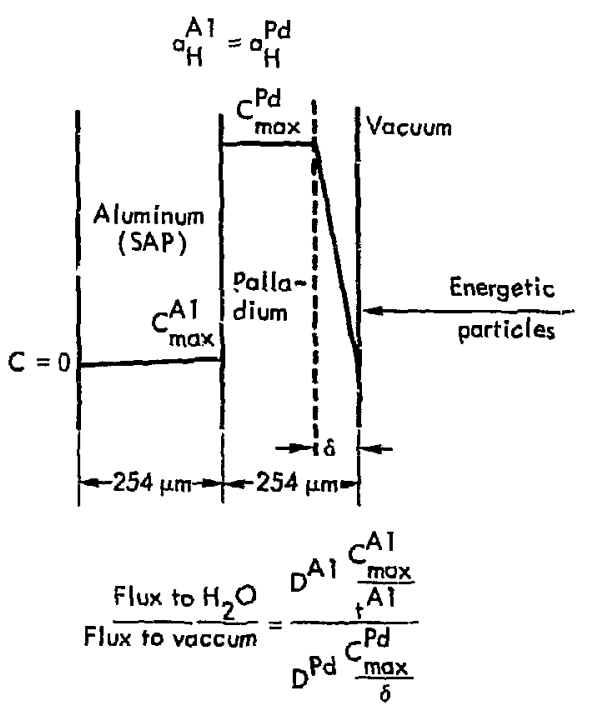

Fig. C-6. Permeation of laminated AI-Pd first wall.

sure) is the same. For aluminum, the permeability, $\phi$, is given by $\mathrm{G} 13$

$$
\phi=1.1 \exp \left(-\frac{26,000}{1.987(\mathrm{~T})}\right) \mathrm{m} \ell / \mathrm{cm} \cdot \mathrm{s} \cdot \mathrm{atm} \cdot 1 / 2 .
$$

Therefore, the permeation rate of tritium through a first-wall surface of $10 \mathrm{~m}^{2}$ which is receiving the fluxes of energy and ions mentioned above is

$$
\begin{aligned}
\text { Loss }= & \frac{\phi_{\text {Al }} p_{\text {atm }}^{1 / 2} A_{\text {wall }}}{t_{\text {tube }}} \\
= & 1.1 \exp \left(-\frac{26,000}{1.987(350)}\right) 3.02 \times 10^{-4} \\
& \times\left(10^{5}\right) / 0.0254 \\
= & 7.6 \times 10^{-14} \mathrm{ml} / \mathrm{s} .
\end{aligned}
$$

In one operating year, at $2.625 \mathrm{Ci} / \mathrm{ml}$, the tritium loss $=6.27 \times 10^{-6} \mathrm{ri}$ for the first-wall system. This should be compared with $3.8 \times 10^{6} \mathrm{Ci}$ for either aluminum or palladium when used alone in the jsothermal approximation. Losses for the composite case can be ignored. 
If this behavior is verified experimentally, the coolant system will not receive a significant amount of tritium.

\section{Outgnssing}

Just as every metal will permeate tritium to some extent, every metal which has been exposed to tritium will also outges tritium when exposed to air. Metals with large solubilities or which have been implanted with tritium can be especially bad in this respect. When the FERF reactor or other major system components are disassembled. we should expect trit:am to be released to the atmosphere surrounding the equipment. The amount of tritium released at this time could be substantially more than that escaping during normal operation.

The amount of tritium outgassing can be estimated by assuming the release of a 100 -monolayer equivalent from the metal surfaces. This is probably too high an estimate for metal surfaces that have not been bombarded with energetic particles, but it is probably low for those that have been. If we assume that the metal surface exposed is $120 \mathrm{~m}^{2}$, then $1.2 \mathrm{~g}(12,000 \mathrm{Ci})$ escapes. The main hall would become tritiumcontaminated.

We can reduce this problem by well over $90 \%$ by intentionally outgassing the equipment before disassembly. G14 For the FERF reactor, this might be done by shutting off the injectors and runining a hot fluid through the first-wall coolant tubes while maintaining a good vacuum. For the fuel-system piping and fuel-processing equipment, electric heaters can be attached to the exterior of the components while they are varuum-pumped. After this, the equipment could be disassembled. On the basis of our (incomplete) present knowledge, we would predict a release of less than $0.1 \mathrm{~g}$ tritium $(1000 \mathrm{Ci})$.

\section{Stcondary Containment}

The rather large tritium inventory on the cryopumping surfaces could be a problem if there were a severe failure of the vacuum system. Such a failure is not expected, but because of the extraordinary neutron fluences parts of the machine will receive, a sudden mechanical failure is not impossible. A large air leak into the FERF reactor would cause the condensed deuterium and tritium to evaporate quickly.
Explosive mixtures could form and the hot injector parts could cause detonation. $\mathbf{G 1 5}$ To preclude the occurrence of this accident situation, the room in which the machine is located will be backfilled with nitrogen. A large leak cannot create an explosive mixture.

This technique wili be applied to all areas containing components having a large tritium inventory. The three areas in this category, the large hall in which the FERF reactor is located, a much smaller room containing the fuel-reprocessing equipment, and the fuelstorage vault, all will have nitrogen atmosphere, and the walls will be steel-lined and leak-proof. 'st the event of a large tritium release, the nitrogen will become contaminated. but no explosion or fire can occur and no tritium can escape.

F'sewhere in a room, also metal-lined but isolated from the others, is equipment for removing tritium from the nitrogen. This general scheme is shown in Fig. G-7 and an example of a secondarily contained wall penetration between adjac ent rooms is shown in Fig. G-8. This room constitutes the main tritium recovery system (TRS). Because it will usually contain small inventories of tritium at low concentrations, its atmosphere is air.

An accurate estimation of the design of the TRS is not yet possible. Aithough the use of small systems for special applications has been common practice for many years, no one has built a TRS which would have the capacity and removal efficiency required of this one. The main TRS will probably consist of activated carbon beds to trap organic vapors and protect the catalysts, heat exchargers (heaters), hot catalyst beds, more heat exchangers (coolers), molecular sieve beds to remove tritiated water formed in the catalyst beds, water injectors, and more molecular sieve beds to redry the nitrogen. All of the molecular sieve beds should be regenerable, and equipment to accomplish this must be included in the TRS room. These particular operations are presently a little beyond the state of the art. They are also the largest processing operations in the entire facility.

Cost estimates for a TRS are based on very long extrapolations of what we think we can do today. For example, a system with a $0.028-\mathrm{m}^{3} / \mathrm{s}(60-\mathrm{cfm})$ capacity was recently installed at Monsanto Research Corporation's Mound Laboratories at a cost of 


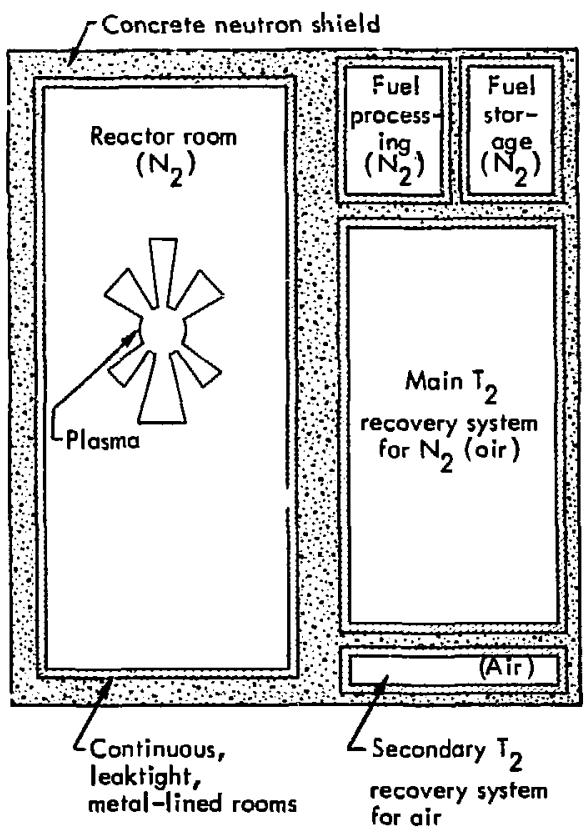

Fig. G-7. Secondary containment of tritium using blanketing atmospheres.

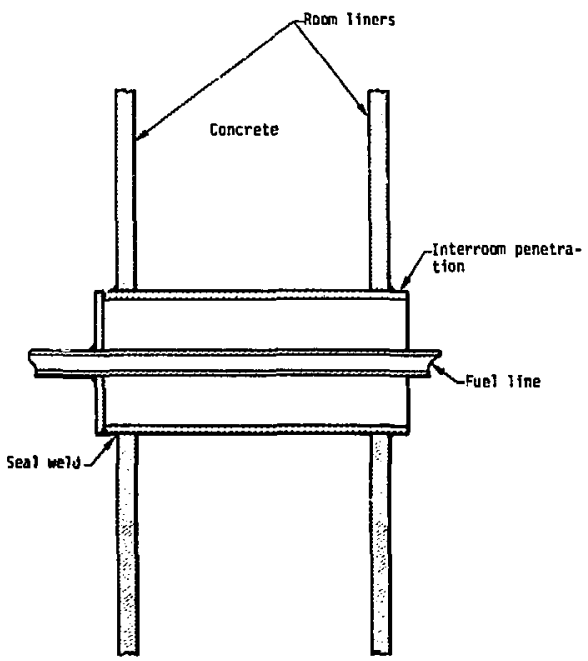

Fig. G.8. Scheme for providing secondary containment for inter-room fuel line. slightly over a quarter million dollars. G16 A system being constructed for Sandia Livermore Laboratories will have a $0.08-\mathrm{m}^{3} / \mathrm{s}$ capacity and should reduce tritium content to $500 \mu \mathrm{Ci} / \mathrm{m}^{3}$. Its cost (including instrumentation and control), has been estimated at between $\$ 400,000$ and $\$ 1,000,000$. ${ }^{17}$ The capacity needed to process the gas in the FERF's nitrogenblanketed rooms should be about $50 \mathrm{~m}^{3} / \mathrm{s}$. The diameter of each of the molecular sieve beds is $4 \mathrm{~m}$, and a total of 20 would be required. A schematic of the system needed for FERF is shown in Fig. G-9.

For two reasons, a small TRS in s:other isolated room is provided to maintain the ai? quality in the main TRS room. First, the main TRS may need total shutdown for major repair or modification. It then couldn't keep the tritium content at safe levels in its own room to permit repair operations, even if tl:e workers wore special protective clothing. Also, i: would be convenient to get into the TRS room to do minor repairs while it is operating.

Some high-energy neutrons will escape from the reactor and pass through the nitrogen blanket. Some of the neutrons in the nitrogen may form tritium through the ${ }^{14} \mathrm{~N}(\mathrm{n}, \mathrm{T})^{12} \mathrm{C}$ reaction. The amount of tritium gas produced this way would be $20 \mathrm{ml} / \mathrm{yr}$ $(50 \mathrm{Ci} / \mathrm{yr})$, an amount easily handled by the main TRS.G18

\section{Accidents}

\section{Failure of First Wall}

The first wall is cooled with high-pressure, fastflowing cold water contained in thin-walled tubs tubes. These first-wall tubes will receive massive neutron doses, will undergo severe sputtering, will experience several kinds of radiation damage along with helium embrittlement from $(n, \alpha)$ reactions, and will have thermal stresses imposed on them. A tube might fail, the plasma would immediately go out, and the plasma vacuum chamber would partially fill with water. Because the water would becoine tritiumcontaminated, some tritiated water would enter the coolant loop. Therefore, coolant water cann tt be circilated directly to a cooling tower, but must first go through a water-water heat exchanger located within the biological tritium shield. Thus, if the coolant water should beccme contaminated, it can be removed and disposed of safely. 


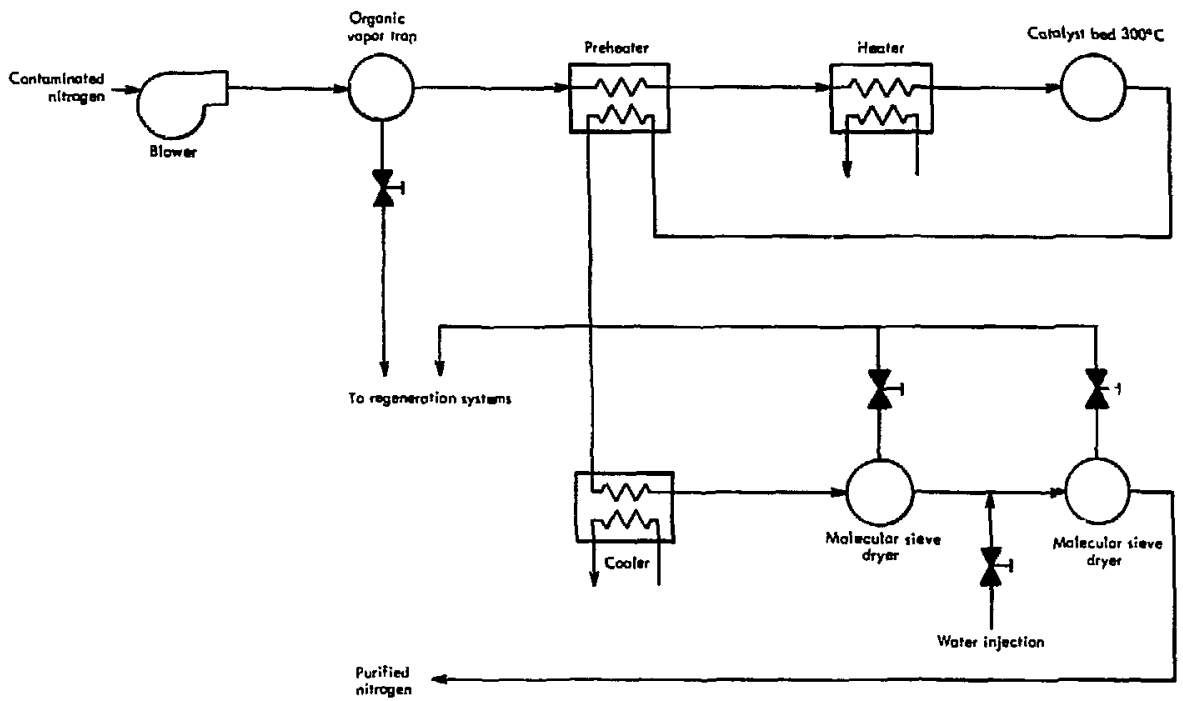

Fig. G-9. Tritium recovery systen module (10 modules required).

The same considerations apply to the water that would squirt into the main chamber if a first-wall tube should fail. The volume of water would :e much less, but its tritium content would be higher. This water might be drained from the reactor and either pumped directly on to moleculnr sieves or evaporated and trapped by the main TRS drying beds.

\section{Failure of Magnet Shield}

Broted water will probably be used in the neutron shield and as coolant. By removing contamilused solution and adding fresh, its tritium content can be kept below $50 \mathrm{Ci} / \mathbf{l}$. As implied earlier, a leak in the coolant circulation syst $\mathrm{m}$ could release hazardous quantities of radioactivity to the main hall in the form of tritiated water. As with failure in the first wall, a substantial water spill could be handled by the main TRS. Additional cleanup and repair w suld require trained workers in protective clothing, with due consideration for other forms of radioactivity that might be present. Special suits which provide an effectiv. $10^{5}$ reduction in tritium exposure have been developed recently at CEN/Saclay. G19

\section{Failure of Vacuum System in FERF Reactor}

Failure of the vacuum system implies leakage of nitrogen and some oxygen into the system; however, no explosion or fire cculd occur. The palladium diffusers could be used to recover some (but not all) of the fuel which became contaminated. Sinilarly, any tritium that leaked into the nitrogen blanket surrounding the equipment would be removed by the main TKS. However, tlis cleanup would not produce a significant increase in the amount of waste already teing pu-fuced, but the radioactivity level of the waste thus produced would be higher than normal.

\section{Failure of Distillation System}

The distillation columns are the only comperents of the entire fuel-handling system that run above atn ospheric pressure. They receive no neutron radiation damaze, and the chance that this will either burst or suffer weld failure is extremely remote. There is a passibility of a valve failure, but this can be dealt with.

The still and other cryogenic components will be protected by a vacuum jacket and therefore have 
built-in secondary containment. Loss of vacuum in the jacket could result either from nitrogen leaking in from the cutside or DT leaking out from the column interior. The former case would be of little consequence other than causing some repair work because it would result in rapid boiling of the entire distillation apparatus inventory. A pressure surge could result which would either be contained by the inherent strengti of the system or, more likely, be relieved by rupturing an all-welded rupture disk which would abort the system to a large reservoir. Filling the vacuum jacket with DT would cause loss of vacuum with the same consequences: the equipment would require disassembly for repair.

Loss of vacuum in the jacket of one stili will rerylt in automatic transfer of operation to the other still, but will not necessarily result in a tritium release. If tritium did escape, it would be into the nitrogen atmosphese being processed by the main TRS.

\section{Anticipated Tritium Releases}

In a facility that is nominally totally enclosed, there are only two ways for tritium to escape. One is permeation through the stainless steel room liners followed by escape to the atmosphere through cracks or seams in the building. The other is during deliberate flushing of ais locks to allow people to move out of the radionctive materiaks areas.

Tritium should not escape through coolants because of the secondary lonps used for isolation. Also, all heat exchangers will operate at quite low temperatures, will be constructed of low-permeability copper alloys, and will have relatively small surface areas.

If we attain state-of-the-art performance with the main TRS, the partial pressure of tritium in the nitrogen will be around $10^{-8} \mathrm{~atm}$. Using the steady-state permeability equation $\mathbf{6} 20$

$$
\phi=2.9 \times 10^{-3} \text { exp } \frac{-12,000}{\text { RT }} \frac{\mathrm{ml}}{\mathrm{cm} \mathrm{satm}^{1 / 2}} \text {, }
$$

and assuming $4 \times 10^{4} \mathrm{~m}^{2}$ of stainless steel wall liner with a $1-\mathrm{cm}$ thickness, the tritium loss by permeation is calculated to be $2 \mathrm{mg} / \mathrm{yr}(20 \mathrm{Ci} / \mathrm{yr})$.
Larger releases will come from flushing personnel and equipment locks. If we assume that three small air locks of $10 \mathrm{~m}^{3}$ each are used three times per day (once per shift) as people move out of the tritium handling area, then $33,000 \mathrm{~m}^{3}$ of contaminated gas would be released per year from the source. Suppose the volume of the large air lock used for moving major pieces of equipment is $3000 \mathrm{~m}^{3}$. and that it is used threc times per year. The total centaminated volume of gas released from this suurce would be about $42,000 \mathrm{~m}^{3}$. With a contamination level of $10^{-8}$ tritium, $420 \mathrm{ml}$ ( $1100 \mathrm{Ci}$ ) of tritium would he released per year.

\section{MONITORING AND PERSONNEL PROTECTION}

Two kinds of tritiunı monitoring will be appropriate for the FERF. The first measures the tritium contents of the effuents from the building stack. These gases would normally include only air from the offices, control room, work shops, and air locks. During periods of planned shut-down, when all the tritium is safely stored in the vault, it might also include air from the radioactive materials area if the recirculating nitrogen is replaced by once-through air ventilation. Our atmospheric modeling does not make this option attractive unless outgassing prior to disassembly is very effective.

The monitoring equipment will consist of ion chambers with readouts for stack-effluent concentrations and integrated total release. Such systems, coupled to appropriate alarm systems, already exist. G21 Monitors at locations throughout the facility will give people working in the area information on their local air quality. These self jontained monitors will give instantaneous local tritium concentrations and will have alarm levels at two tritium concentrations. These individual monitors will be interconnected to a master control and alarm panel in the control room. Systems such as this are also presently in use and are found to be quite satisfactory.

Personnel protection includes much of what has been discussed so far; however, in the following context it is restricted to protective clothing. Protective clothing must be worn whenever a large tritium inventory is present in the system components. This is selr-evident for those areas where nitrogen blanketing 

G20. J. $\therefore$ Collins and J. C. Turnbull, Vacuum 11 , I 14 (1961).
G21. T. B. Rhinehammer and P. H. Tamberger, Iritium Control Technology, J.S. AEC Rept. WASH-1269 (1973). 



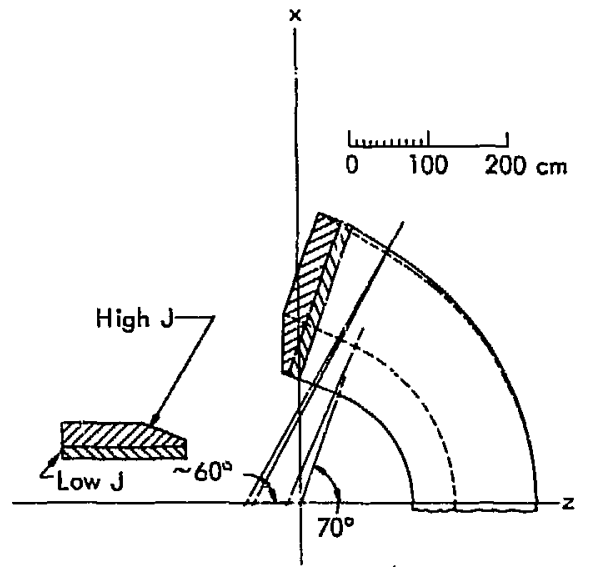

Fig. H-1. Cross section of magnet.

Table H-1. Parameters for low- and high-field magnets

\begin{tabular}{lcr}
\multicolumn{1}{c}{ Parameter } & $\begin{array}{c}\text { Low-field } \\
(\mathrm{T})\end{array}$ & $\begin{array}{c}\text { High-field } \\
(\mathrm{T})\end{array}$ \\
\hline B (z=0) & 3.75 & 5.00 \\
$\mathrm{~B}_{\max }$ (axial) & 7.62 & 10.15 \\
R (dimensionless) & 2.03 & 2.03 \\
B at conductor (max) & & \\
Crossover & 9.20 & 12.27 \\
Turnaround & 9.61 & 12.81 \\
\hline
\end{tabular}

The maximum conductor fields, 9.61 and $12.81 \mathrm{~T}$ are greater than the target values of 9 and $12 \mathrm{~T}$ by $6.7 \%$. The average current density in the high-field magnet is $2.3 \mathrm{I} \mathrm{kA} \cdot \mathrm{cm}^{-2}$. Minor adjustments of conductor location should achieve the desired field vaiues in a more detailed design.

The radial well depth (the ratio of $B$ at the outermost line threading plasma to $B$ at the center) is 1.007 in the $z=0$ plane. This is its lowest value; the field is minimum-B everywhere between the mirrors.

A plot of the field lines which envelope the plasma is shown in Fig. H-2. The surface which envelopes a circle of $25-\mathrm{cm}$ radius at $z=0$ at the mirror limit becomes approximately an ellipse in cross section. The ellipse has $75.33-\mathrm{cm}$ major radius and $3.84 \mathrm{~cm}$

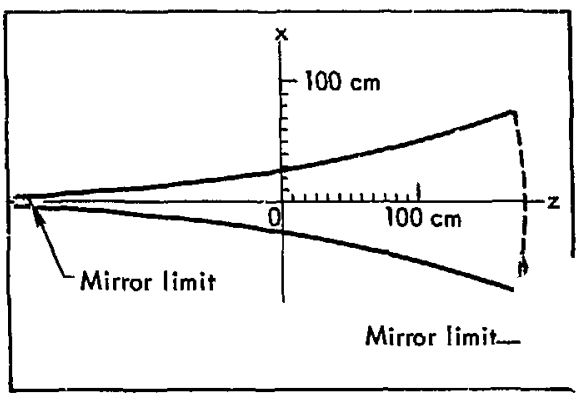

Fig. H-2. Envelope of outermost field lines surrounding plasma.

minor radius.

The forces on the magnet were calculated by simulating one of the coils with a single filament at the current-weighted averaged locus of the original 46 filaments. This filament was broken at several places, and the induction at the breaks was calculated with MAFCO. The cross-product, $\vec{I} \times \vec{B}$, was then taken to get the force per meter length. The distribution of forces in the $x-, y-$, and $z$-directions on one-fourth of a coil is shown in Fig. H-3.

\section{STRUCTURAL DESIGN}

\section{Forces}

The structure for the FERF magnet system is designed for the high-field or $5.0 \mathrm{~T}$ central-field case. In the following calculations, no attempt is made to

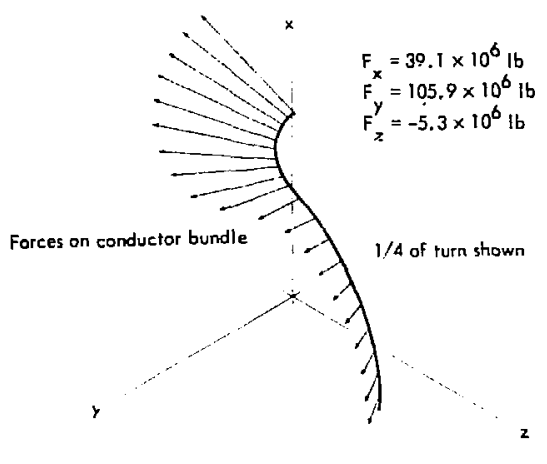

Fig. H-3. Forces on conductor bundle. 
optimize the design. The solution proposed is not the only approach to the problem and may not be the best. It is clear that the complex three-dimensional structural problem of this coil structure requires extensive analysis before a final design can be made. These calculations are intended only to demonstrate feasibility within the confines of space available for magnet structure. It may be desirable to increase the magnet size somewhat to reduce the unit stress in some areas and simplify the structure problem. In Fig. H-3, only the total net force on one quarter of the current bundle is shown for the algh field or 5.0-T centralfield situation. For design purposes, the forces that load both the small- and large-radius turn must be known. These forces are given in Table H-2.

Table H-2. Forces on magnet.

\begin{tabular}{lccc} 
Force & $\begin{array}{c}\text { Total force } \\
\left(\times 10^{6} \mathrm{lb}\right)\end{array}$ & $\begin{array}{c}\text { Force on large- } \\
\text { radius tums } \\
\left(\times 10^{6} \mathrm{lb}\right)\end{array}$ & $\begin{array}{c}\text { Force on small- } \\
\text { radius tums } \\
\left(\times 10^{6} \mathrm{lb}\right)\end{array}$ \\
\hline $\mathrm{F}_{\mathrm{X}}$ & 39.09 & 14.52 & 24.57 \\
$\mathrm{~F}_{\mathrm{y}}$ & 105.92 & 62.1 & 43.82 \\
$\mathrm{~F}_{\mathrm{z}}$ & -5.33 & 10.87 & -16.2 \\
\hline
\end{tabular}

The above tabulated values must be doubled to get the total forces on the entire coil.

Throughout the following calculations, the magnetic loads are assumed to be transmitted to the magnet case by compression, and the superconducting winding itself is not loaded in tension. In reality, some of these loads may be taken by the superconductor if the winding was placed with the proper amount of preload and if some movement was possible before the winding contacted the case. Because these conditions are difficult to achieve in a complex magnet of this design, the more conservative approach is used.

\section{Required Structure}

First consider the forces in the $\mathrm{x}$ direction that place a bending moment on the magnet case. If all the load were taken by the rectangular cross section of the coil case that lies in the $y, z$ plane, the wall thickness would be excessive and both plasma volume and space available for beam injection would be reduced. Therefore, the magnetic load is divided be-

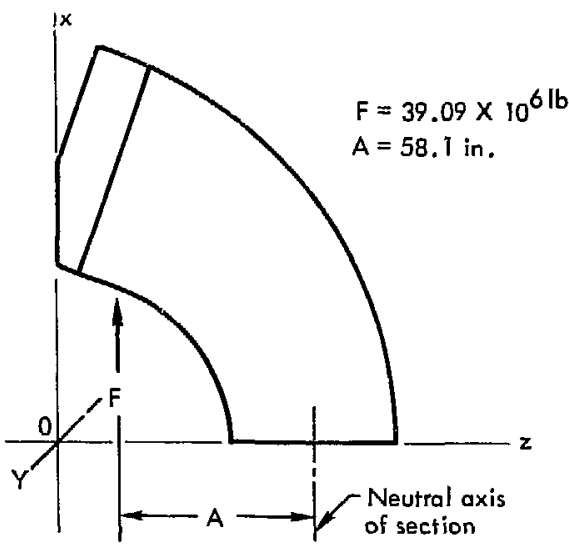

Fig. H-4. Cross section of coil in $x, z$ piane.

tween tine coil case and a semielliptical beam structare on each side of both Yin-Yang coils. The detailed analysis of a structure of this type is dependent upon the daflection of the various components and can involve a rather complex solution. For simplicity, it is assumed that the load is equally divided between the two support nembers.

The moment due to $F_{X}$ on the large radius is

$$
M_{L}=14.52 \times 10^{6} \times \frac{110 \mathrm{~cm}}{2.54}=628.8 \times 10^{6} \text { in. lb. }
$$

The moment arm of $110 \mathrm{~cm}$ is determined by force distribution on the large-radius turn. The arm is $170 \mathrm{~cm}$ from the neutral axis for the small rat'ius.

$M_{S}=24.57 \times 10^{6}\left(\frac{170}{2.54}\right)=1644.5 \times 10^{6}$ in. lb.

Total moment is $2273.3 \times 10^{6} \mathrm{in.} \mathrm{lb}$.

Average moment arm $=\frac{2273.3 \times 10^{6}}{39.09}=58.1$ in. or $148 \mathrm{~cm}$.

This is shown as $\mathrm{A}$ in Fig. H-4.

The cross section of the coil case in the $y, z$ plane is assumed rectangular and has the dimensions shown i Fig. H-5. 



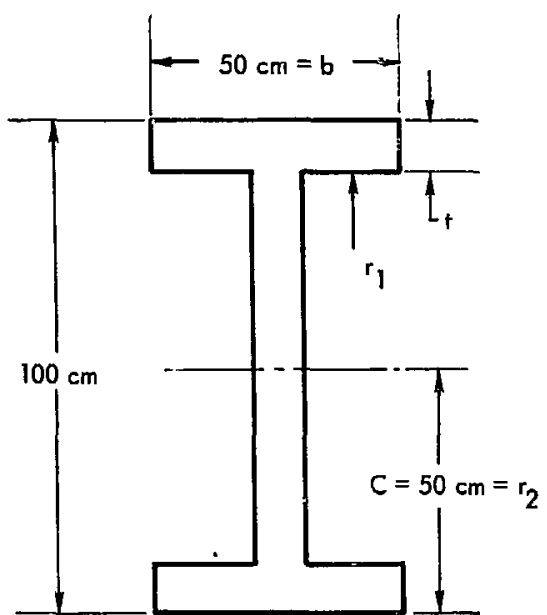

Fig. H-7. 1-beam dimensions.

or, in this case,

$I=2 b \int r^{2} d r$ or $I=\frac{2 b}{3}\left[r_{2}^{3}-r_{1}^{3} \cdot\right]$

Therefore,

$$
\left(r_{1}=14.8 \text { inch, and } t=r_{2}-r_{1}=4.9\right. \text { inch. }
$$

Due to the curvature, the centroid has moved toward the inner surface of the beam, and the inner flange would be thicker than the outside flange if the surface stress in each flange were the same. The best solution to this stress problem may be to eliminate the curved I-beam and increase the wall thickness of the case in the areas of maximum stress. This would require an increase in radius of the small-radius turns on each Yin-Yang coil, which in turn would increase the space between the large-radius turns. Some changes in the magnet would be necessary to maintain the required magnetic field profile.

Forces in the $y$ direction on the large-radius tums are restrained by the large C-clamp structures that lie in the $x, y$ plane (see Fig. H-8). The $\Sigma F_{y}=62.1$ $X 10^{6} \mathrm{lb}$ and is nearly uniform over an area of 9,642 in. $^{3}$, i.e., over the area in one quadrant. The unit pressure, $P$, is then

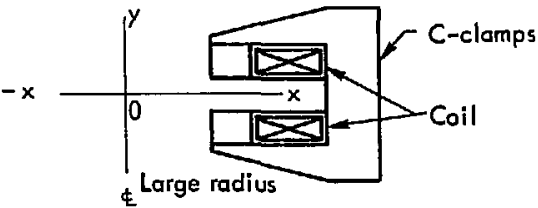

Fig. H.8. C-clamp supporting coil.

$$
P=\frac{62.1 \times 10^{6}}{9,642}=6,440 \mathrm{psi}
$$

A simple approximate check on this pressure can be made by a direct calculation of magnetic field "pressure":

$$
\mathrm{P}=\frac{\mathrm{B}^{2}}{1.72}
$$

where pressure is in psi and $B$ is in $k G$. The field between the large-radius turns for a mirror radio of 2 is $50 \mathrm{kG} \times 2+100 \mathrm{kG}$ is $100 \mathrm{kG}$. Therefore,

$$
P=\frac{100^{2}}{1.72}=5,813 \text { psi. }
$$

Consider a tapered cantilever that is 1 -in. thick and has a span equal to that of the largest C-clamp, i.e., 134 in. 0 : $3.4 \mathrm{~m}$ (see Fig. H-9).

Then the bending moment is $M=\frac{F \ell^{2}}{2}=\frac{6440\left(134^{2}\right)}{2}=57.8 \times 10^{6}$ in. $l b$, and the moment of inertia for a stress of $10^{5}$ psi is

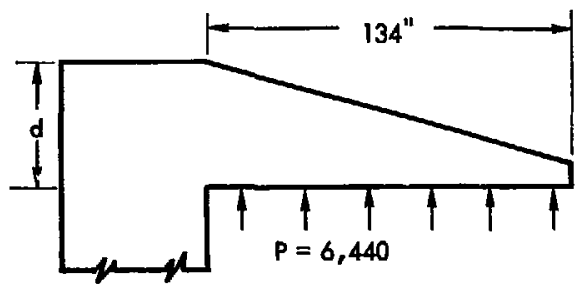

Fig. H-9. Tapered cantilever. 


$$
\mathrm{I} / \mathrm{C}=\mathrm{M}=\frac{57.8 \times 10^{6}}{10^{5}}=578 \mathrm{in}^{3}
$$

For the rectangular section 1 -in. wide, the depth of the beams, $d$, can be determined

$$
1 / C=(1 / 6) b d^{2}=(1 / 6)(1)\left(d^{2}\right)
$$

so that

$$
d=2 \sqrt{(6)(578)}=58.9 \text { in. }
$$

This would be the maximum depth of C-clamps. As the span decreases so would the depth $d$.

The vector sum of the forces in the $-\mathrm{z}$ direction plus the forces in the $y$ direction on the small-radius turns place a hoop stress on the semicircular cap (Fig. $\mathrm{H}-10$ ). Since the forces on each side in the $y$ direction cancel one another, only the $\mathbf{z}$ forces are considered.

$\Sigma F_{z}=16.2 \times 10^{6} \times 2=\frac{2(55 \mathrm{~cm})}{2.54^{2}}(225)\left(P_{z}\right)$

$P_{2}=8,433$ psi.

(The magnetic field "pressure" of $12 \mathrm{~T}$ is 8,370 psi.) Consider this as a hydraulic pressure in a cylinder, and solve for the wall thickness for a stress of $10^{5} \mathrm{psi}$.

$$
\sigma=\frac{P D}{2 t}
$$

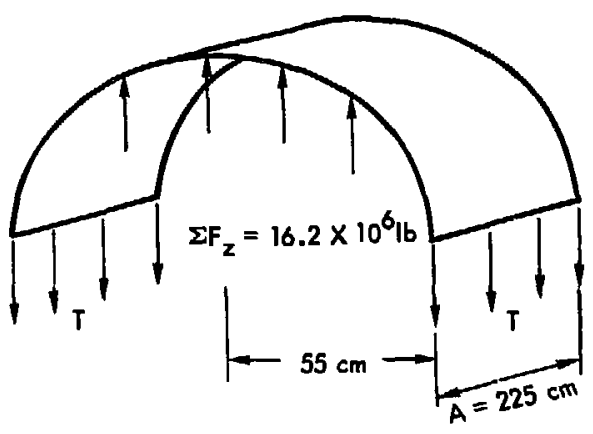

Fig. H-10. Stress on semicircular cap. where $\sigma$ in the stress $P$ is pressure in psi, $D$ is the i,d. of the winding, and $t$ is the thickness of the cise in inches.

Then

$$
D=\frac{(55)(2)}{2.54}=43.3 \mathrm{in} .
$$

and

$$
t=\frac{P D}{2 \times 0}=\frac{(8433)(43.3)}{2 \times 10^{5}}=1.8 \mathrm{in} .
$$

The thickness, 1.8 in., is the minimunı needed to retain the $z$ forces on the small-radius turn. The wall thickness must be increased in the $x, z$ plane so that it will also take the loads in the $y$ direction.

If the load

$$
F_{y}=43.8 \times 10^{6} \mathrm{lb}
$$

is distributed throughout the cross section of the coil sase in the $x, 2$ plane, the thickness would be

$$
t=\frac{F_{y}}{\text { (perimeter) }(0)}=\frac{\left(43.8 \times 10^{6}\right)(2.54)}{2(225+45) \times 10^{5}}=2 \mathrm{in} .
$$

This wall thickness must be added to the 1.8-in. thickness for a total cap thickness of 3.8 in. Actually, some of the load on the outer cap would be trans. mitted to the side wall of the cap and the coil and the unit stress would be reduced.

The sum of the forces in the $z$ direction on the barge-radius tum is $10.87 \times 10^{6} \mathrm{ib}$. The load is applied by compression to the curved surface of the coil cover, but since the coil width in the $x$ direction is only $45 \mathrm{~cm}$, it will be distributed to the coil cover that lies in the $x, z$ plane and the total ares of the coil from in the $x, y$ plane. The cross section in Fig. H-1I is the box section of the coil case at the plane of transition between the small and large turns. The total area of this cross section is

$A_{x y}=[45+2(12.7)](225+11+14)$

$$
-(45)(225)=7,475 \mathrm{~cm}^{2}=1,159 \mathrm{in}^{2}
$$


Therefore, the unit stress on this area due to the $F_{2}$ of $10.87 \times 10^{6}$ would be

$$
\sigma=\frac{10.87 \times 10^{6}}{1159}=9.378 \mathrm{psi} .
$$

The forces in the $z$ direction on the large-radius turns also place a bending moment on the cross section of the coil case in the $y, z$ plane. This moment is opposite in sign to that imposed by the $F_{X}$ on this same cross section and would reduce the stress in this ares.

The net attraction force between the two Yin-Yang coils is a compressive load on the interface when the coils are separated. An estimote of the cross-sectional area limits the unit stress to 5,000-psi maximum.

\section{Materials for Magnet Structure}

High strength, toughness, and low magnetic permeability are necessary propertios of the magnet structural material. Several candidates are listed in Table $\mathbf{H}-\mathbf{3}$. Or these, the Armco 21-6-9 stainless steel appears to be a good choice. The yield and tensile strengths are very high, yet the material exhibits good toughness at cryogenic temperatures. The plane strin fracture toughness has been measured to be $108 \mathrm{ksi} \cdot(\text { in. })^{-1 / 2}$,

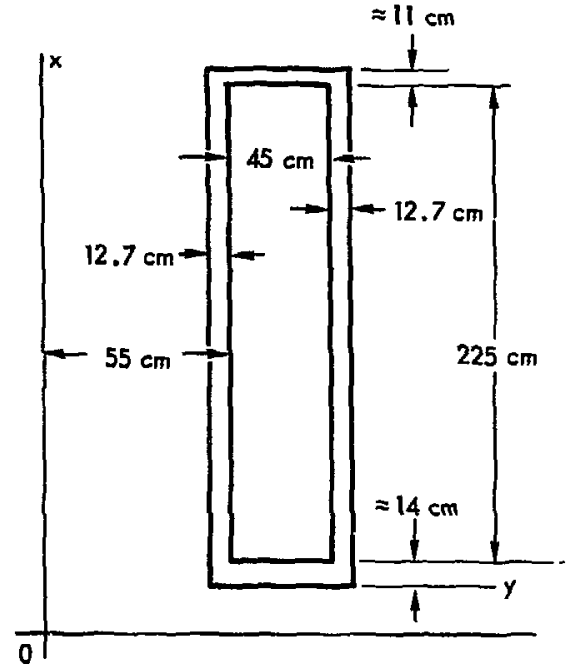

Fig. H-1 1. Cross section of the coil case.

which is comparable to mild steel at room temperature. Further cryogenic data for this alloy is listed in Table $\mathrm{H}-4$ and the composition is given in Table H-5. The alloy is fusion weldable, tut for good ductility a high nickel filler is suggested in large weldments.

Table H-3. Properties of various metals.

\begin{tabular}{|c|c|c|c|c|c|c|c|}
\hline Material ${ }^{3}$ & $\begin{array}{l}\text { At room ti } \\
\text { Yield } \\
\text { strength } \\
\text { (ksi) }\end{array}$ & $\begin{array}{l}\text { Tensils } \\
\text { stringth } \\
\text { (ksi) }\end{array}$ & $\begin{array}{l}\text { At } 4 \\
\text { Yield } \\
\text { strength } \\
\text { (ksi) }\end{array}$ & $\begin{array}{l}\text { K } \\
\text { Tensile } \\
\text { strength } \\
\text { (ksi) }\end{array}$ & $\begin{array}{c}\text { Aren reduction } \\
\text { or elongation } \\
\text { at } 4.2 \mathrm{~K} \\
(\xi)\end{array}$ & Permeability & Comments \\
\hline 304 SS & 30 & 80 & $40-80$ & 235 & 55 & $1.01 \cdot 10$ & $\begin{array}{l}\text { Precipitates martensite } \\
\text { at low temperature; } \\
\text { ferrites in weld. }\end{array}$ \\
\hline 316 SS CF-8M & 35 & 90 & $40-80$ & 215 & 60 & 1.01 & $\begin{array}{l}\mathrm{K}_{\mathrm{ic}}=79 \mathrm{ksi} / \overline{\mathrm{in}} \text {; } \\
\text { ferrites in weld. }\end{array}$ \\
\hline Kromare 55 & 33 & 69 & 81 & 128 & 30 & 1.002 & Fully austenitic. \\
\hline $21-6-9 \mathrm{SS}$ & 55 & 95 & 196 & 245 & 20 & 1.002 & $\begin{array}{l}\mathrm{K}_{\mathrm{ic}}=108 \mathrm{ksi} \sqrt{\mathrm{in}} \text {; } \\
\text { fully austenitic. }\end{array}$ \\
\hline 7039 T64 Al & 55 & 65 & 65 & 53 & 16 & 1.000 & \\
\hline $310 \mathrm{SS}$ & 38 & 82 & 105 & 188 & 55 & low & \\
\hline $\begin{array}{l}\text { Cold-worked } \\
\text { MP35N }\end{array}$ & 252 & 274 & 332 & 349 & 38 & low & Cold worked $60 \%$ \\
\hline
\end{tabular}

aReference to a company or product name docs not imply approval or recommendation of the product by the University of California or the U.S. Atomic Energy Commission to the exclusion of others that may be suitable. 
Table H-4. Typical properties of 21-6-9 stainless steel at cryogenic temperatures ${ }^{a, b, c}$

\begin{tabular}{ccccc}
\hline $\begin{array}{c}\text { Test temperature } \\
\left({ }^{\circ} \mathbf{F}\right)\end{array}$ & $\begin{array}{c}\text { Ultimate tensile } \\
\text { strength } \\
(\mathbf{k s i})\end{array}$ & $\begin{array}{c}\mathbf{0 . 2 \%} \text { yield strength } \\
\text { (ksi) }\end{array}$ & $\begin{array}{c}\text { Elongation in 1" } \\
(\%)\end{array}$ & $\begin{array}{c}\text { Reduction of area } \\
(\%)\end{array}$ \\
\hline 75 & 103 & 58 & 50 & 70 \\
-110 & 134 & 87 & 59 & 71 \\
-320 & 203 & 150 & - & 24 \\
-423 & 245 & 196 & 15 & 20.5 \\
\hline
\end{tabular}

${ }^{a}$ Armco product data

b Tested in transverse direction

${ }^{c}$ Annealed 4-3/4-in. thick slab.

Another attractive material is MP35N Multiphase because it has extremely high strength with good ductility. The alloy (composition in Table $\mathbf{H}-5$ ) has a face-centered cubic matrix of cobalt and nickel in which the chromium and molybdenum are soluble at elevated temperatures. A face-centered cubic structure will persist upon cooling, but cold working forms small platelets of a hexagonal, close-packed structure. These small platelets are responsible for the high strength of the worked material because they impede further deformation. The large amount of cold work required limits the size of fabricated structures and also precludes welding. This probably makes the material impractical for the main coil housing. However, braces and fixtures up to 2 -in. thick would be fabricated. By judicious use of these materials, design stresses of $100 \mathrm{ksi}$ in the magnet structure may be permitted.

\section{FABRICATION OF WINDINGS}

The magnets will be wound on a three-axis winder which has a vertical and one horizontal axis capable of complete rotation. The remaining horizontal axis will partially' rotate. Because of the cross-sectional dimensions of the magnets and the large radius swing during windim $\mathrm{F}_{\mathbf{5}}$ technicizns will be positioned on a mobile platform in the winding area. To accommodate increasing radial swing and conductor-layer build, tensioning and conductor pay-off equipment would be mounted on a platform capable of sidewise translation and advancing elevation adjustment.

Solder joints will be fabricated to provide maximum shear area as well as resistance to peel when under load. (This was done with the Baseball II magnet by jacketing the lapped conductor in the joint area.)

Insulation material will be a polyimide or polyimideglass laminate. This is the most radiation-resistant organic material presently available. Interlayer insulation will consist of slotted laminate sheets, and interturn insulation will consist of spaced, laminated dots captured by film strip.

The variable winding space between turns will be maintained by machined or laminated copper or polyimide wedges and rectangular bars. The crosssection of the conductor is considerably larger than that required for imbedment of the superconductor filament. Therefore, the filaments could be imbedded in a corner of the conductor so that minimum strain

Table H-5. Composition of 21-6-9 stainless steel and MP35N multiphase.

\begin{tabular}{lllll}
\hline \multicolumn{2}{c}{$21-6-9$ SS } & & \multicolumn{1}{c}{ MP35N Multiphase } \\
\cline { 1 - 2 } Element & $\begin{array}{c}\text { Composition } \\
(\%)\end{array}$ & Element & $\begin{array}{c}\text { Composition } \\
(\%)\end{array}$ \\
\hline Carbon & $0.04-0.08$ & Nickel & 35 \\
Manganese & $8.00-10.00$ & Cobalt & 35 \\
Phosphorus & 0.060 max. & Chromium & 20 \\
Sulfur & 0.030 max. & Molybdenum & 10 \\
Silicon & 1.00 max. & & \\
Chromium & $18.00-21.00$ & & \\
Nickel & $5.50-7.50$ & & \\
Nitrogen & $0.15-0.40$ & & \\
\hline
\end{tabular}




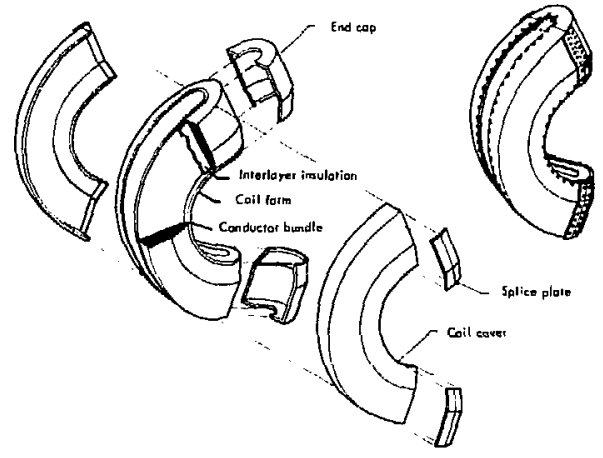

Fig. H.12. Expanded view of magnet.

would result on the filaments during winding.

Layers and turns will be arranged as indicated in

Fig. H-12. The inner winding surface will consist of stackable stainless steel rings which will remain part of the structure. Similarly, rings provided at the outer periphery of the windings will provide for subsequent load transfer to the covers.

\section{MAGNET POWER SUPPLY}

The total magnetic energy is $1400 \mathrm{MJ}$. The operating current in each winding is about $10,000 \mathrm{~A}$.

Figure $\mathrm{H}-13$ is a simplified schematic of a suitable magnet power supply. To reduce the insulation requirements, each winding is divided into four identical sections. This also permits rapid emergency discharge of the magnet. During nomal operation, the $0.06-\Omega$ discharge resistor is short-circuited by the parallel thyristor so that essentially no power is dissipated. However, under fault conditions the following sequence

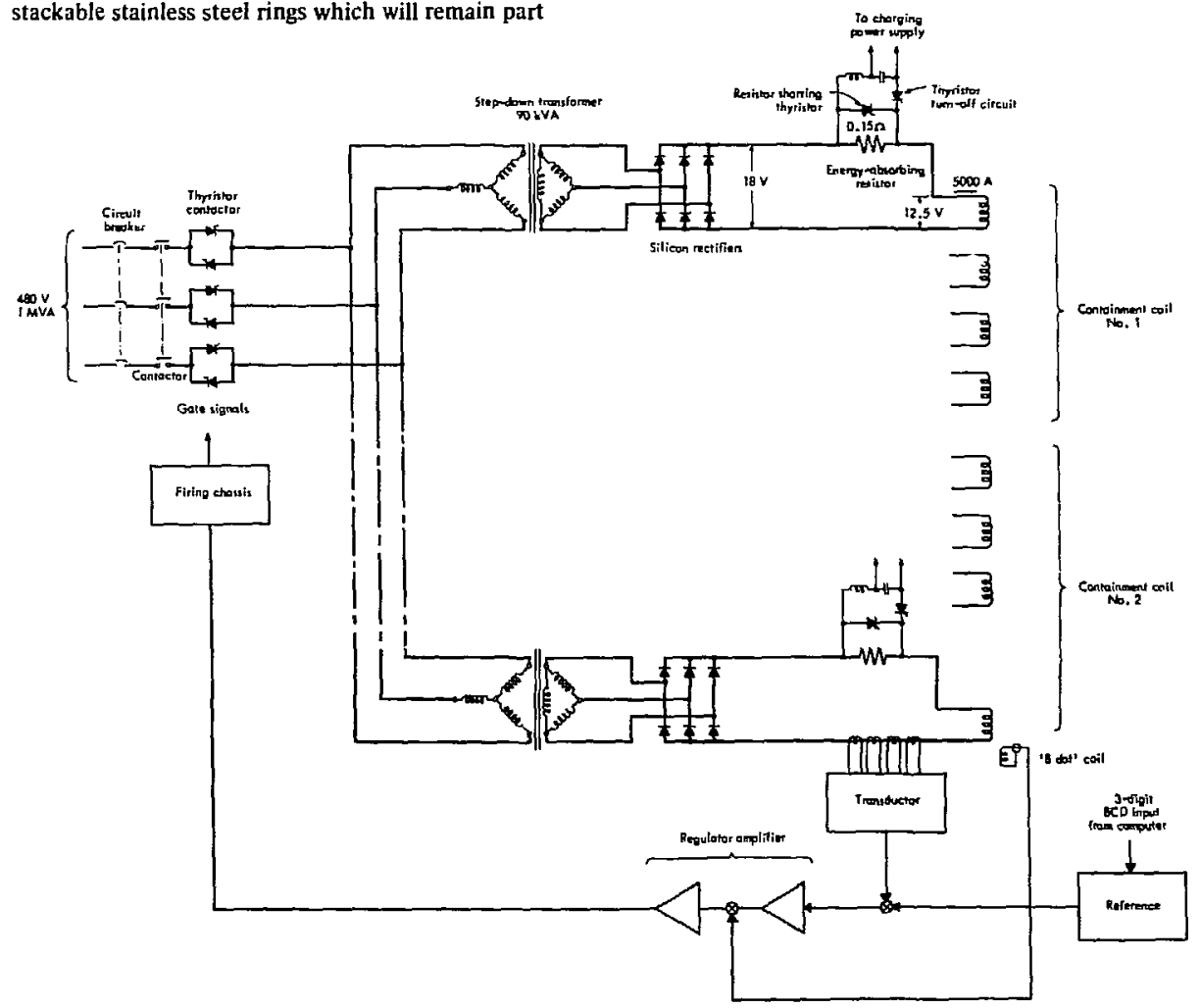

Fig. H-13. Schematic of magnet power supply. 
occurs:

- The magnet power supply is de-energized:

- The commutistor thyristor is fired, thereby opening ine parallel thyristor and allowing the magnet energy te be dissipated in the resistor.

This sequence discharges the magnet in $60 \mathrm{~s}$, a short time compared with the 2000s required for charging.

The rapid discharge requires that the magnet voltage per coil rise from the normal $12.5 \mathrm{~V}$ to $600 \mathrm{~V}$ and that the magnet insulation be appropriate for this voltage. Also, zach of the eight discharge resistors must be capable of dissipating $175 \mathrm{MJ}$ during the $60 \mathrm{~s}$ discharge.

As shown in Fig. H-13, a typical, two-loop, currentregulator circuit actuates the conducting period of the thyristor contactor. The a.c. loop coming from the "B dot" coil must be switched in electronically after the current has reached the operating value and the regulator preamplifier comes out of saturation. Using a standard technique in the art of magnet regulation, this second loop improves the regulator performance by making the time rate of change of field zero.

The presence of a thyristor cuntactor as the regular actuating element permits the use of small, watercooled transformer rectifier units. Because these units can be located close to the magnet windings, it is possible to reduce the length of the low-voltage. high-current, d.c. bussing to the magnet.

To accommodate computer control, the regulator reference uses digital voltage selection. 


\section{Appendix I - Vacuum Pumpin $;$ Requirements}

Cryogenic pumps will be needed within the FERF neutral beam injectors and in the mirror chambers. Mechanical pumps will be used during degassing cycles.

\section{INJECTOR PUMFING SYSTEM}

FERF will have four neutral beam injectors, any three of which will supply $D^{0} T^{0}$ beams of adequate capacity to sustain the plasma. To keep the load on the plasma within tolerable limits, a gas load of 180 Torr $\cdot l \mathrm{~s}^{-1}$ must be pumped for each of the four injectors (see Table B-2). The space available for pumps and the size of the load precludes any method of pumping other than cryopumps.

Each injector will have two cryopumping volumes separated by a tubular impedance which is transparent to the $\mathrm{D}^{0} \mathrm{~T}^{0}$ beam. This arrangement allows $90 \%$ of the gas to be pumped in the first volume at a pressure 12 times higher than the pressure in the volume adjacent to the plasma. To keep neutral beam reionization within acceptable limits, the volume adjacent to the plasma must be held below $3 \times 10^{-5}$ Torr. Pumping the full gas load at the lower pressure would require an impractically large cryopumping system.

Because of lack of space, the conventional chevronshielded cryopump dirangement cannot be used on the injector pumps. The injector pumping system therefore consists of liquid-helium-cooled cryopanels which are shielded from the warm vacuum walls by liquid-nitrogen-cooled surfaces. The liquid nitrogen fills the need for cooling the neutralizer tubes and the gas-flow impedance tubes as well as shadow-shielding the opening to the plasma chamber. Ccaling the internal elements of the beam line with liquid nitrogen permits maximum gas conductance to the cryopanels.

The characteristics of the injector pumps are listed in Table 1-1.

The gas load on the plasma from gas diffusing from the injectors and sireaming from the sources amounts to about $7 \mathrm{~A}$ of diffuse gas and $26 \mathrm{~A}$ of streaming gas for a total of 33 molecular amperes for all four injectors.
Table 1-1. Injector pumping system.

\begin{tabular}{ll}
\hline & Capability \\
\hline $\begin{array}{l}\text { Total cyropanel area for one } \\
\text { injector (one side only): }\end{array}$ & $10^{5} \mathrm{~cm}^{2}$ \\
$\begin{array}{l}\text { Total cryopanel pumping speed } \\
\text { for one injector: } \\
\begin{array}{l}\text { Pressure in the low-pressure } \\
\text { pump space: }\end{array}\end{array}$ & $9 \times \eta^{5} \mathrm{\ell \cdot s}-1$ \\
$\begin{array}{l}\text { Pressure in the high-pressure } \\
\text { pump space: }\end{array}$ & $3 \times 10^{-5}$ Torr \\
\hline
\end{tabular}

\section{MIRROR CHAMBER PUMPS}

The gas loads emerging from the mirrors in the form of high-energy $\mathrm{D}^{+}$and $\mathrm{T}^{+}$ions must also be pumped. The ion current escaping from each mirror is approximately $373 \mathrm{~A}$ of $\mathrm{D}^{+}$and $\mathrm{T}^{+}$, which amounts to a gas load of 32 Torr $\ell \cdot s^{-1}$. The ions will be dumped on water-cooled plates and subsequently pumped on cryopanels cooled to $4.2 \mathrm{~K}$. The pressure that must be held in the mirror chambers is determined by the amount of back-diffusing gas that can be tolerated by the plasma. This amount has been estimated at $5 \mathrm{~A}$ of $D_{2}^{0}$ per mirror of 0.85 Torr $\cdot \ell \mathrm{s}^{-1}$. The required pressure, considering the gas conductance through the mirror duct. is $2 \times 10^{-5}$ Torr. This requires a pumping speed of $1.6 \times 10^{6} \mathrm{Qs}^{-1}$ in the pumping chamber.

To allow continuous operation of the machine, mirror chamber pumps must be designed in modules that can be separately isolated and degassed. There will be 10 to 12 modules per mirror chamber. The characteristics of the mirror chamber pumps are listed in Table $\mathbf{l - 2}$.

The mirror chamber can possibly operate at higher pressure (up to $10^{-4}$ Torr), greatly reducing the required pump capacity. This would result from the ionization of the back diffusing gas by the ion current cxiting the mirrors. The effecti would be a large reduction in the gas conductance from the mirror chamber to the plasma chamber. 
Table I-2. Mirror chamber pumps.

Item Capability

Required cryopanels area for one mirror chamber (one side only):

$4 \times 10^{5} \mathrm{~cm}^{2}$

Total pumping speed, including the effects of liquid-nitrogen-cooled entrance baffles, per mirror chamber:

$1.6 \times 10^{6} \ell \mathrm{s}^{-1}$

Operating pressure:

$2 \times 10^{-5}$ Torr

\section{RECYCLING PUMP}

Because the plasma can be sustained by three of the four injecters, each injector can be shut down in sequence for degassing the cryopanels. After $1 \mathrm{~h}$ of operation, there will be approximately $10^{5}$ monolayers of gas (gas $\sim 30-\mu \mathrm{m}$ thick) on the cryopanels. By closing a valve between the injector and the plasma volume, the cryopanels can be warmed to about $15 \mathrm{~K}$, thereby converting all of the condensed deuterium and tritium back to the gas phase at an equilibrium pressure near 66 Torr. The injector will then be evacuated by ari $1800-\ell-s^{-1}$ mechanical pump to $10^{-2}$ Torr; the cryopanels would then be recooled to $4.2 \mathrm{~K}$ in preparation for reactivating the injector. The degassing cycle would require about 2 to 5 minutes. Time between degassing operations can be varied between a few minutes to sbout $1 \mathrm{~h}$, depending on acceptable tritium holdup.

A 25-inch-diameter manifold will collect gas from all the cryopumps during the sequenced degassing cycles. This operation will require two $1800-\ell \cdot \mathrm{s}^{-1}$ compoi id blower-piston muchanical pumps and isolation valves for all punif " e pumped gas will be processed to remove tielium and to isotopically separate deuterium and tritium. This portion of the sy:tem is described in Appendix G. 
Appendix J - Heat Flux c.apability of the First Wall

\section{DEFINITION OF THE PROBLEM}

Because of the directionality of the irjectors and because the reactor must pass through the startup and plasma-buildup phase where the unattenuated neutrais hit the wall, the maximum thermal flux from these neutrals ina" range from values as high as $\sim 3300$ $\mathrm{W} / \mathrm{cm}^{2}$ for short times to values of $\sim 1000 \mathrm{~W} / \mathrm{cm}^{2}$ for steady state (see Appendix C). Figure 1 (page 5) shows the region within the reactor where the flux is incident.

For FERF, two additional considerations arise in addition to the energy removil:

- The metallurgical effects of neutrals and neutrons should be separated to make analysis less difficult. To do this, the short-range neutrals (those having a 1 - to $10-\mu \mathrm{m}$ burial depth in metals) are intercepted by a sacrificial first wall or inst-wall shield. The effects of the neutrons can then be evaluated in a test nigion immediately on the other side of this wall.

- The cooling of the first wall in FERF, compared to that in a commercial reactor, is not (economically) limited in pressure drop or pumping power. The wali may also be changed several times a year.

To remove the very large energy flux from the first wall, the following methods are among those possible:

- Transpiration cooling.

- Film cooling.

- Film evaporation.

- Ablation cooling.

- Radiation cooling.

- High-velocity, forced, convective cuoling with liquids in the nonboiling and subcooled boiling regimes.

The first three methods have in cammon the lise of a liquid coolant film on the surface of the wall facing the plasma. Plasma-wall interactions with this arrangement would probahly quench the plasma because liquids have high sputtering coefficients. Ablative cooling involves the physical removal of wafl material, and this probably would also quench the plasma. Radiation cooling in this geometry does not solve the problem but merely transfers it to another surface. We have, t.nerefore, focused our attention on the last listed inethac, the use of high-velocity, forted, convective ccoling with liquids in the nonboiling and subcooled boiling regines. This metrod has the additional advantage of f-oducing low wall temperatures and, consequently, higher allowable streis.

Also, if the walls facing the plasma are cool, aluminum alloys may te considered for their constiuction. These materials produce relatively short half-life species after neutmon irrediation, making the handling, disposid, and replacement of first-wall structures less of a problem.

In forced convective cooling, the maximum heat tlux may be limited by any one of several engineering constraints, ir:/luding:

- Allowable structural stres;es.

- Maximum allowable structural temperature.

- Coolant pumping power requirements.

- Coolane pressure drops.

- Critical treat flux in bciling liquids.

- Practical tube diameters, thicknesses, and lengths.

These constraints are examinet in the following sections.

\section{FIRST-WALL CONCEPT}

The concept selected for this study involves an array of closely packed tubes or thin, corrugated sheets joined to furm a wall of how chamels. This highty cooted wall can be used as the first-wall shield and placeri in front of the containment vessel so as to shield it from erosion. To the an attractive concept for FERF, the first-yoll shield should be substantially easier and less costly to tabricate, install, maintain, and replace than the first wafl or the front part of the containment vessel itself, partizularly if frequent replacement proves to be necessary.

\section{THERMAL STRESS LIMITATICNS}

Bec-use of the iniph heat fuxes expected in FERF, the design of the tube wati is strongly influenced by thermal stress. The the;mal stress, $v_{t h i}$, in a rituwalled tube is: 


$$
v_{\text {th }}=\left(\frac{\alpha E}{2(1-\nu)}\right) \Delta \mathrm{T}_{w}
$$

where $\Delta T_{W}=$ temperature across the tube wall, $\alpha=$ coefficient of thermal expansion, $E=$ modulus of elasticity, and $\nu=$ Poisson ratio. The surface heat flux $q$ and the conduction equation determine $\Delta T_{w}$ so that

$$
\sigma_{\text {th }}=\left(\frac{\alpha E}{2(1-\nu) k}\right) q \delta
$$

where $q=$ local heat flux, $k=$ thermal conductivity, and $\delta=$ tube wall thickness. The quantity in parentheses is material- and temperature-dependent and is a useful guide for material selection.

Actually, the total stress is complicated by the details of specific designs but is not less thar the sum of the hoop stress and the thermal stress. Therefore,

$$
\sigma_{\text {total }} \geqq \frac{\mathrm{pD}}{2 \delta}+\left(\frac{\alpha \mathrm{E}}{2(1-\nu) \mathrm{k}}\right) \mathrm{q} \delta
$$

where $p=$ pressure in the tube, and $D=$ tube diameter. However, as an aid for preliminary material selection we will assume that thermal stress dominates and plot two different criteria relative to thermal stresses.

Using Eq. (J2) with property data taken at room temperature yields Fig. J-1 a plot of thermal stress vs thermal flux for representative materials. This plot serves to divide the materials into two categories: those that are clearly thermal-stress limited at flux levels of interest and therefore unacceptable, and those materials that are acceptable.

We see that stainless steel, vanadium, and titanium all have a sharp sensitivity to thermal nux and are not likely first-wall materials. The acceptable alloys are those of tantalum, molybdenum, niobium, aluminum, and the special aluminum alloy SAP. However, the use of aluminum alloys is uncertain, not because of high thermal stresses, but simply due to low allowable working temperature. For niobium. molybdenum, and tantalum, the stresses are well below the yield point at $1000 \mathrm{~W} / \mathrm{cm}^{2}$.

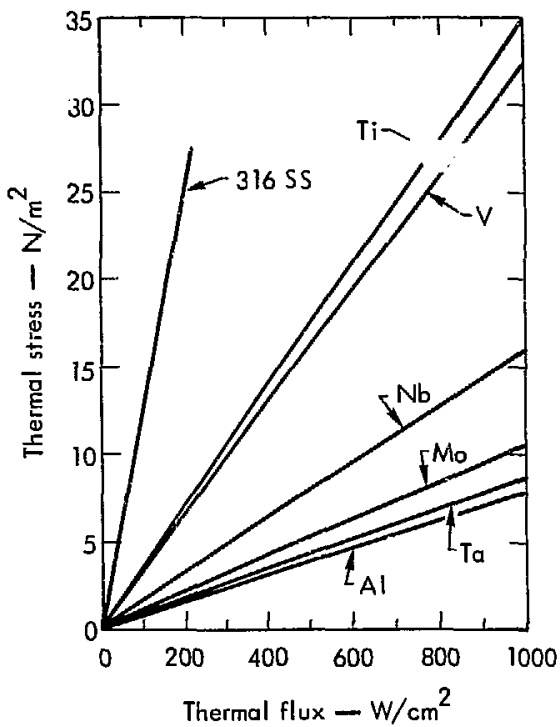

Fig. J-1. Thermal stress vs thermal flux for some materials of interest in FERF first-wall application.

An even more comprehensive material property parameter, $M^{*}$, can be used to compare potential first-wall materials

$$
\mathrm{M}^{*}=\left(\frac{2 \sigma_{\mathrm{y}} \mathrm{k}(1-\nu)}{\alpha \mathrm{E}}\right)=\frac{\sigma_{\mathrm{y}}}{\sigma_{\mathrm{th}}} \mathrm{q} \delta
$$

where $\sigma_{y}=$ short-time tensile yield stress. This parameter is most useful for the case of very high heat fluxes in thin-walled tubes where the thermal stresses dominate all other stresses including the hoop stresses. Under these conditions, the ratio $\left(\sigma_{y} / \sigma_{t h}\right)$ is essentially equal to the structural safety factor. If we use the same safety factor for all materials and the same tube-wall thickness, $\delta$, then $M^{*}$ is directly proportional to the maximum heat flux the material can safely handle.

Reliable and comparable values of the material properties (particularly the tensile yield stress) for the various materials as a function of temperature are difficult to obtain. We have tried to be as consistent as possible in the choice of the data used to calculate the $M^{*}$ values plotted in Fig. J-2, but some uncertainties remain. Nonetheless, the qualitative trends 


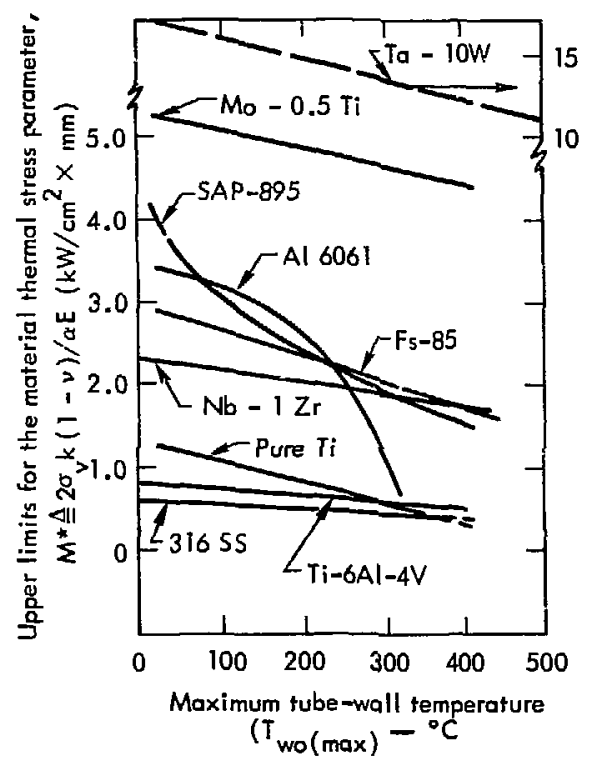

Fig. J-2. Comparison between various promising first-wall shield materials in terms of a material property parameter related to thermal stresses in thin-walled tubes.

shown in Fig. J-2 should be correct. In particular, the high yield stresses of Ta-10W and Mo-0.5Ti, coupled with favorable property data (as measured by the low slopes of Fig. J-1), result in the highest heat-flux capabilities. The niobium and aluminum alloys form an intermediate group of materials which are still possible choices, particularly if the wall temperatures can be kept low. The alloys of titanium and stainless steel and commercially pure vanadium are the least attractive of the materials investigated for these high heat-flux applications.

\section{TRANSIENT BEHAVIOR}

In addition to problems of thermal stress, the choice of wall materials is influenced by the transient (startup) behavior of the reactor. Thermal fluxes on the wall during this time interval, or during a time interval when the plasma is lost but fuel injection continues, are as high as $3300 \mathrm{~W} / \mathrm{cm}^{2}$. It will be assumed that with this high flux the critical heat flux for convective heat transfer may be exceeded (although, as will be shown later in this appendix, this does not appear to be the case if the Rousar correlation is correct). The critical heat flux is the point at which there is a sharp reduction of the local heat transfer coefficient caused by replacement of the liquid by vapor at the heated surface. Critical heat flux sets the upper limit for convective heat transfer, and burnout (tube melting) can occur.

We are concerned with the time-temperature history of the tube wall, particularly the uter surface of the tube facing the plasma. There are two parameters that may be used to gage the teminerature of tlie tube wall. The first parameter is

$$
\widetilde{\Delta \mathrm{T}_{\infty}} \triangleq\left[\mathrm{T}(\theta)-\mathrm{T}(0) \mathrm{I}_{\infty}=\frac{\mathrm{q} \theta}{\rho \mathrm{C}_{\mathrm{p}} \delta}\right.
$$

where $\theta$ is timc, $\rho$ is density, and $\mathrm{C}_{\mathrm{p}}$ is the specific heat. This is the exact solution of the time history of the temperature of a thin plate of infinite thermal conductivity without heat removal. The second parameter, the Fourier number, is useful for the cas is wherc the thermal conductivity is finite.

$$
\mathrm{F}=\alpha^{\prime} \frac{\theta}{\delta^{2}}=\frac{\mathrm{k}}{\rho \mathrm{C}_{\mathrm{p}}} \frac{\theta}{\delta^{2}}
$$

which includes $\alpha^{\prime}$, the thermal diffusivity. It can be shown that for finite thermal conductivity at the point $\mathbf{x}$ into the tube wall,

$$
T(\theta)-T(0)=f\left(F, \overline{\left.\Delta T_{\infty}, x / \delta\right)}\right.
$$

This is plotted in Fig. J-3.

Table $\mathrm{J}-\mathrm{I}$ sums up the $\overline{\Delta \mathrm{T}_{\infty}}$ values and the Fourier numbers for a representative tube-wall thickness, $\delta$, of $1 \mathrm{~mm}$ and a heat flux of $q=3300 \mathrm{~W} / \mathrm{cm}^{2}$.

By using these values of $F$ and $\overline{\Delta T_{\infty}}$ for different times, $\theta$, the temperatures of the front face $T_{f f}$ ran be determined from Fig. $J-3$ by noting that the $x / \delta=0$ line refers to the front face, the dashed line is for $\overline{\Delta T}_{\infty}$, and the ratio of their two intercepts on the ordinate is the factor by which $\overline{\Delta T_{\infty}}$ is to be multiplied to obtain the temperature of the front face.

The front-face temperature rise as a function of time is plotted in Fig. J-4. We see that the aluminum 


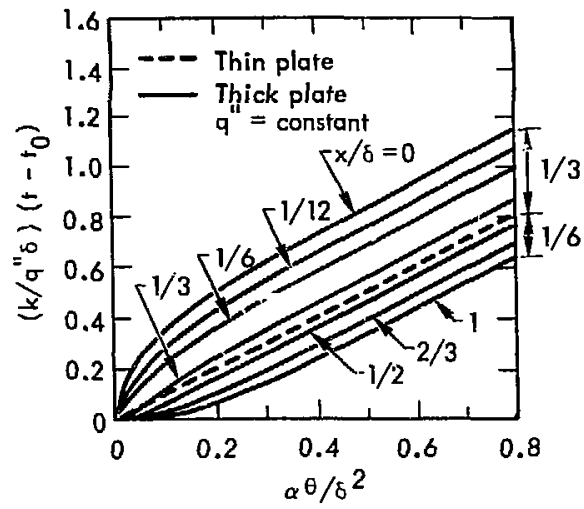

Fig. J-3. Temperature response of finite-thickness plate $(0 \leqslant x \leqslant 8)$ with insulated rear face $x=\delta$ after sudden exposure to constant heat input at $x=0$.

alloys have only about $48 \mathrm{~ms}$ before the front face starts to melt (the melting point of aluminum is $660^{\circ} \mathrm{C}$ ) while the tantalum alloys have over $216 \mathrm{~ms}$. The principal point to be made from these results is that it is more reasonable to provide as much time for transient plasma buildup or loss as possible by choosing materials with high melting points, such as tantalum, in preference to aluminum. This is particularly true for very high heat-flux cases, since there is considerable uncertainty about the critical hest flux under these conditions.

\section{PRESSURE DROP AND PUMPING POWER CONSTRAINTS}

For the steady-state fux, it is desirable to keep the temperature of the first-wall shield low to maximize allowable stress and thus maximize heat flux capability. The decrease in thathes allowate stress temperature is illustrated in Fig. J-2 since it is the dominant cause of the decrease of $\mathrm{M}^{*}$. The maxim am temperature of the outer surface of the tube at the exit is given by:

$\mathrm{T}_{\mathrm{w}(\max )} \triangleq \mathrm{T}_{\mathrm{wo}(\mathrm{ex})}=\mathrm{T}_{\mathrm{B}(\mathrm{in})}+\Delta \mathrm{T}_{\mathrm{B}}+\Delta \mathrm{T}_{\mathrm{film}}+\Delta \mathrm{T}_{\mathrm{W}}$

where $\Delta \mathrm{T}_{\mathrm{B}}=\mathrm{T}_{\mathrm{B}(\mathrm{ex})}-\mathrm{T}_{\mathrm{B}(\mathrm{in})}=$ fluid bulk temperature rise, inlet to exit and $\Delta T_{\text {film }}=\left(T_{b}-T_{w i}\right)_{e x}=$ temperature rise from the bulk fluid to inner tube wall and $\Delta \mathrm{T}_{\mathrm{w}}=\left(\mathrm{T}_{\mathrm{wo}}-\mathrm{T}_{\mathrm{wi}}\right)_{\mathrm{ex}}=$ temperature rise across the wall. These temperatire variations are shown in Fig. J-5. We have no control over the $\Delta T_{w}$ for a given material and wall thickness since it is proportional to the heat flux. Consequently, to minimize $T_{w(\max )}$. we must minimize $T_{B(i n)}, \Delta T_{b}$, and/or $\Delta T_{\text {film }}$.

For this design, liquid water has been selected as the coolant, and the lowest possible value of $T_{b(i n)}$, about $0^{\circ} \mathrm{C}$, has been used. To keep the $\Delta \mathrm{T}_{\mathrm{B}}$ and $\Delta \mathrm{T}_{\text {film }}$ low, the high flow velocities illustrated in Fig. J-6 are required. This leads to high pressure drops, $\Delta \mathrm{p}$, and high pumping power. The pressure drop, $\Delta p=f g^{2} / 2$ Dip is plotted versus the flow rate parameter, $\mathbf{G} \triangleq \rho u$, in Fig. J-7. Converting these pressure drops to pumping power required per square meter of the surface area of the first-wall shield which faces the plarina gives

$$
\frac{P_{\text {pump }}}{A_{f w}}=\frac{\pi D_{i}}{4 L} \frac{G \Delta p}{\rho}=\frac{\pi f}{8} \frac{G^{3}}{\rho^{2}}
$$

Table $\mathrm{J}-\mathrm{l}$. Values of the Fourier number and tube-wall temperature as a function of time for material selected materials (time. 0 , is in seconds)

\begin{tabular}{lccccccc}
\hline Item & $\begin{array}{c}\text { SS } \\
316\end{array}$ & $\begin{array}{c}\mathrm{Nb} \\
\mathrm{FS85}\end{array}$ & $\begin{array}{c}\text { Mo } \\
\text { Mo-0.5Ti }\end{array}$ & $\begin{array}{c}\mathrm{Al} \\
606 \mathrm{I}\end{array}$ & $\mathrm{Ti}$ & $\mathrm{Ta}$ & $\mathrm{V}$ \\
\hline $\begin{array}{c}\text { Fourier No. } \\
\mathrm{F}\end{array}$ & $4.84 \theta$ & $27.0 \theta$ & $43.0 \theta$ & 70.00 & 7.300 & 29.600 & 12.00 \\
$\overline{\Delta \mathrm{T}_{\infty}}$ & $4400 \theta$ & 145000 & 129000 & 135000 & 142000 & 138500 & $1 ! 2000$ \\
\hline
\end{tabular}




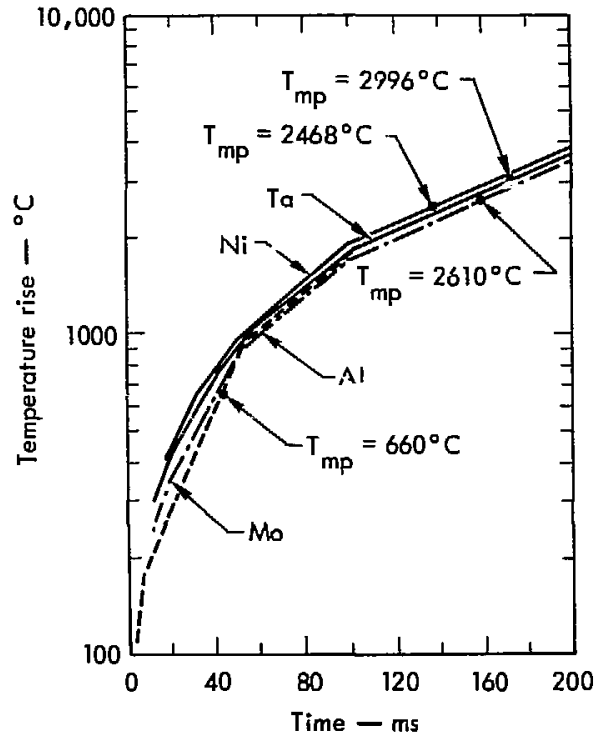

Fig. J-4. Surface temperature of the tube wall vs time for constant flux input of $3000 \mathrm{~W} / \mathrm{cm}^{2}$ withoul heat removal.

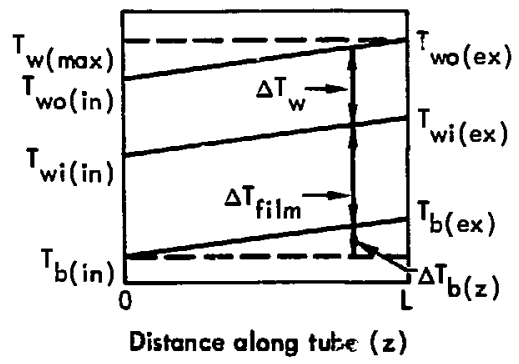

Fig. J-5. Variation of the fluid and wall temperature along a tube with uniform axial heat input.

\section{DESIGN FOR FERF}

For the reference design calculasions, we have chosen $D=10 \mathrm{~mm}, \delta=0.5 \mathrm{~mm}$, and $L=1 \mathrm{~m}$ for a Iepresentative coolant tube. An inlet water pressure of 280 psi was chosen because it is an even multiple of one of the pressures used in the critical heat Bux correlations which will be discussed later in this appendix. To illustrate the calculational procedure in arriv-

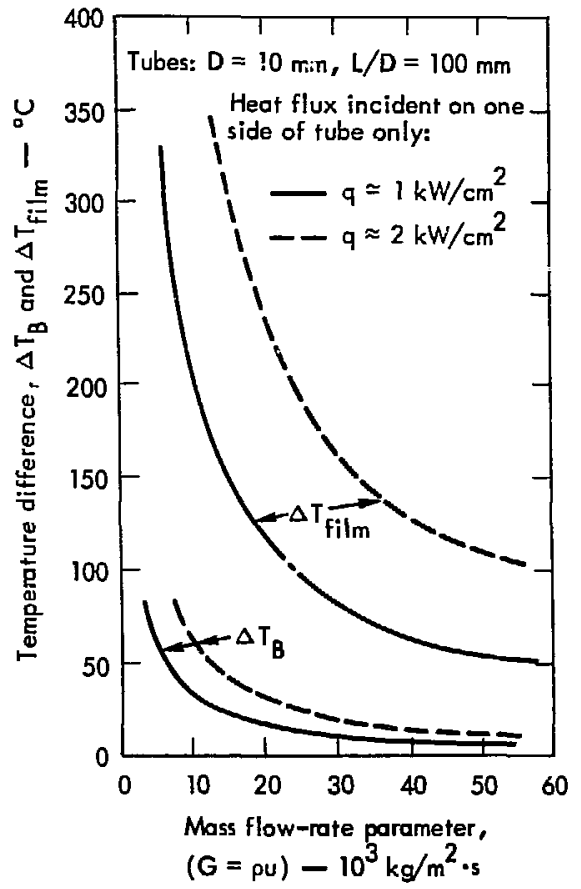

Fig. J-6. Reduction of bulk and film temperatures with increasing coolant moss flow rate.

ing at the maximum allowable heat flux, data for $\mathrm{Nb}-\mathrm{I} \mathrm{Zr}$ have been used in the following figures.

Figure J-8 shows how the total stress (thermal plus hoop stress) varies with heat flux for three different tube-wall thicknesses. The hoop stress. which is the intercept at $q=0$, increases as the tube-wall thickness decreases. However, the total stress is lowest for the $0.25-\mathrm{mm}$ tube wall for heat fluxes above $\sim 600 \mathrm{~W} / \mathrm{cm}^{2}$. We chose a $\mathbf{6}=0.5 \mathrm{~mm}$ because tubes of this diameter are easy to fabricate and to allow for the erosion caused by sputtering.

In Fig. J-9, we have replotted these dala for $\delta=0.5 \mathrm{~mm}$ as a function of the maximum wall temptrature at the tube exit for three typical values of the bulk temperature rise. If we superimpose the allowable stress $\left(2 / 3 o_{y}\right)$ vs temperature curve on this plot, we obtain the locus of possible maximum operating points.

For each bulk temperature rise, there is a particular value of the mass fiow parameter. G. and the allowable 


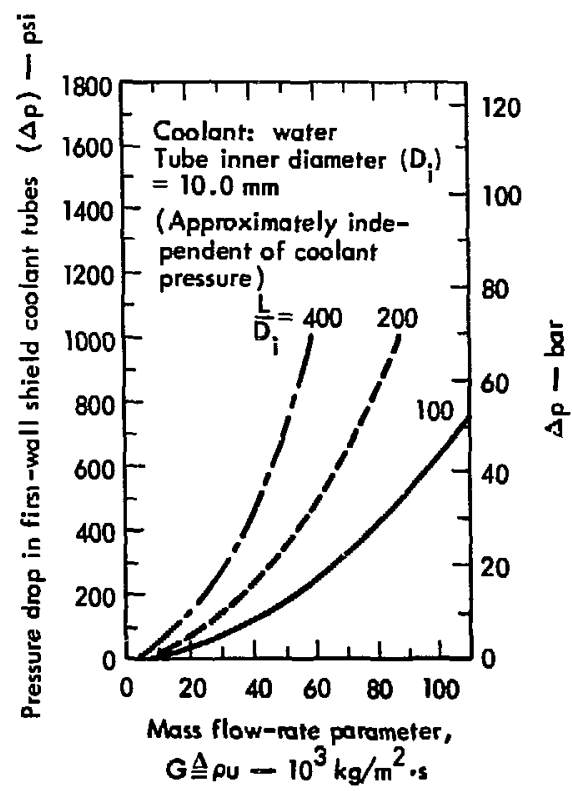

Fig. J-7. Pressure drop in round tubes using water coolant in the nonboiling flow regime.

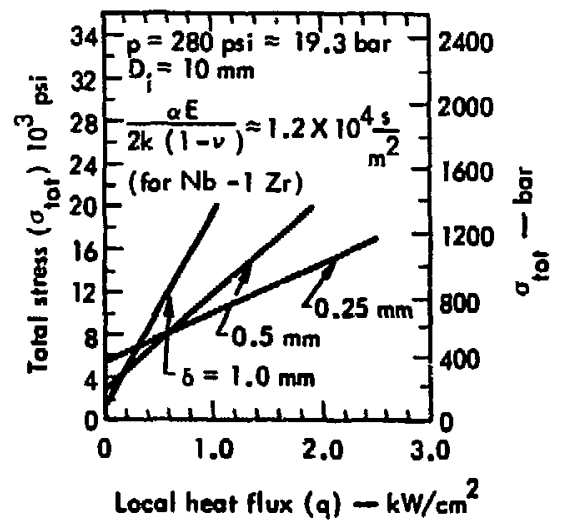

Fig J.8. Total sties an a function of thetmal nux for three difrerent lube thicknesies.

ileat nux. $q$. at cacth intersection point. These values are replotted in Fig. J-10. This stress-limit curve forms the upper boundary for the maximum allowable heat nux for the material being investigated.

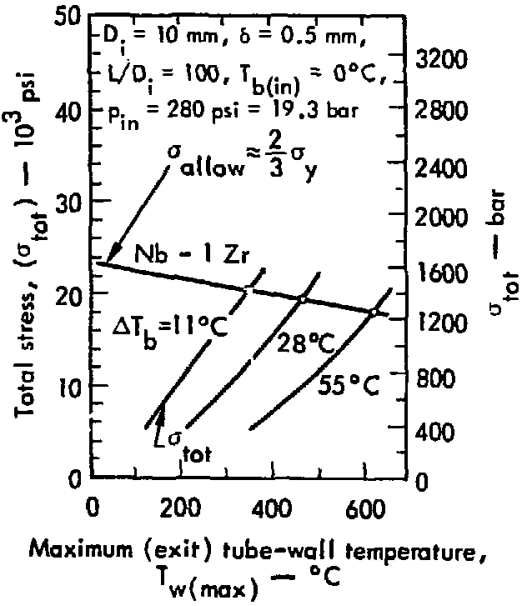

Fig. J-9. Total stress and allowable stress variations with temperature of $\mathrm{Nb}-12 \mathrm{r}$ tube wall. The intersection points define the maximum operating conditions for each case.

Several other constraints can be shown on this type of "performance map." For example, a second constraint is that on the fractional pressure drop. The upper limit on the fractional pressure drop is $\Delta p / P_{\text {in }}=1.0$, which occurs at $G \simeq 64,000 \mathrm{~kg} / \mathrm{m}^{2} \mathrm{~s}$ for the conditions of Fig. J-10. This is shown along with a limit boundary for $\Delta p / p_{i n}=0.5$ (at $G=43,500 \mathrm{~kg} / \mathrm{m}^{2} \mathrm{~s}$ ). The latter value is a more practical engineering upper limit because it allows for additional pressure drops in the bends, headers, and other piping necessary to connect the tubes in the first-wall shield to the water pump. The $\Delta p$ constraint forms the right-hand boundary of the permissible oparating regine of Fig. J-I0.

\section{CRITICAL HEAT FLUX}

A third engineering constraint for liquid coolants is that of the critical heat fux for dryout or bumout. Data are limited for critical heat flux at the very high values of mass flow rate $\left(G>10,000 \mathrm{~kg} / \mathrm{m}^{2} \mathrm{~s}\right)$ in which we are interested. However, Rousaj ${ }^{\prime \prime}$ developed some corrclations using Aerojet data on critical flux whes water is used as a cnolant in this high mass fow 


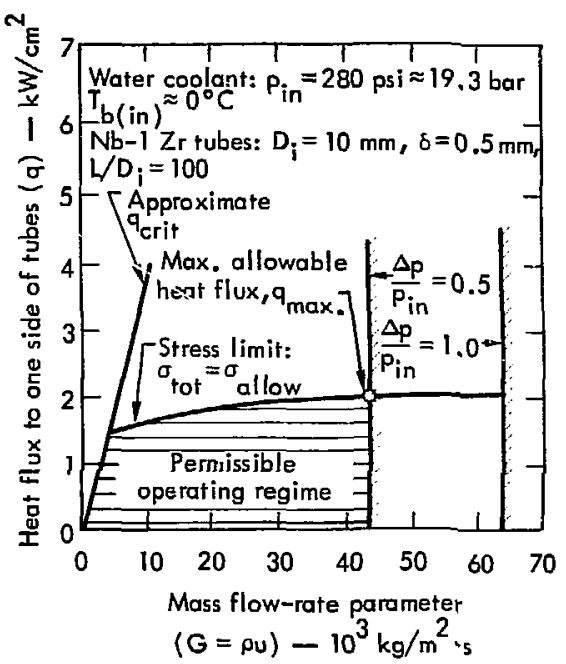

Fig. J-10. Typicil performance map showing the permissible operating regime for the reference first-wall design using $\mathrm{Nb}-\mathrm{I} \mathrm{Zr}$ tubes.

regime. The best fit to these data is shown in Fig. J-11. The dramatic difference between Rousar's correlations and others such as MacBeth's $\mathbf{J} 2$ is quite evident. However, the data on which the MacBeth and other similar correlations are based seem to be for values of $\mathrm{G} \lesssim 7000-8000 \mathrm{~kg} / \mathrm{m}^{2} \mathrm{~s}$ while the Aerojet data is at the higher mass flows.

On that basis, we have tentatively accepted the higher $q_{\text {crit }}$ values based on the Aerojet data as valid for our application. These $q_{\text {crit }}$ values are plotted on the performance map of Fig. J-10 and form the minimum mass flow boundary of the permissible operating regime for the reference design.

It can be seen that the maximum allowable heat flux for $\mathrm{Nb}-\mathrm{IZ} r$ is at the intersection of the stress constraint curve and the pressure drop curve. The water is in the nonboiling regime at this point, far below the critical heat flux curve. The maximum heat flux is $\sim 2 \mathrm{~kW} / \mathrm{cm}^{2}$ with $\mathrm{Nb}-1 \mathrm{Zr}$ tubes. At this condition, the pumping power is $\sim 380 \mathrm{~kW} / \mathrm{m}^{2}$ of first-wall surface area.

Note that the stress-limit curve in Fig. J-10 is quite flat for $\mathrm{Nb}-1 \mathrm{Zr}$ because the allowable stress is a weak function of temperature in this temperature region. As a result, it is possible to reduce the heat flux

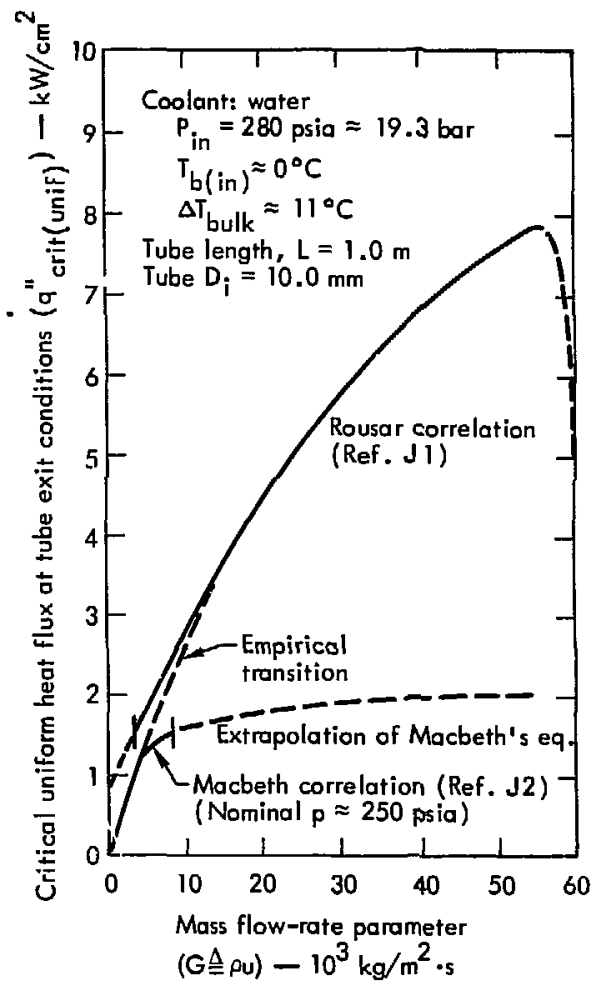

Fig. J-11. Critical heat-flux correlations for uniform heat huxes on round tubes with water coolant.

slightly and save a substantial amount on the pumping power. For example, at $\mathrm{G} \simeq 29,000 \mathrm{~kg} / \mathrm{m}^{2}-\mathrm{s}$, the maximum allowable $q$ is still about $1.9 \mathrm{~kW} / \mathrm{cm}^{2}$, while $\Delta \mathrm{p} / \mathrm{p}$ is reduced to 0.25 . This cuts the pumping power required to $120 \mathrm{~kW} / \mathrm{m}^{2}$, about one-third of the previous value.

Note that as we reduce the mass flow, the subcooled boiling regime is reached. This regime is where most industrial applications operate to obtain the low fractional pressure drops and pumping powers required for good plant efficiency. However, for an experimental fusion facility, we are not strongly constrained in pumping power, and consequently we can operate at the higher values of $G$ in the nonboiling regime up to some economic limit on the pumping system costs and/or on the electric power available to run the pumps. 


\section{COMPARISIJN OF MATERIALS}

The maximum allowable heat flux capabilities for all the materials investigated are shown in the bar chart of Fig. J-12. The basic equations and the materials data used are given in Annex 1 to this Appendix, the solution method in Annex 2, and a numerical example in Annex 3. Clearly, Ta-10W and Mo-0.5Ti are superior.

The upper limit for heat flux for each material is based or short-time tensile property data where we use two thirds of the yield stress. For long-time operation, we have insufficient data on neutron damage and other factors affecting the allowable working stresses to permit choosing a design safety factor. As a rough guess, we suggest that one third the allowable yield stress be used.

All of these maximum heat flux values are well below the critical heat flux curve except for Ta-10W. Hence, all materials except Ta-10W are operating in the nonboiling regime at this maximum heat flux condition, provided that the Rousar correlation is valid.

Note that the maximum tube-wall temperatures for all the materials were below the values where creep effects become dominant. For example, the highest temperature for the $\mathrm{Ta}-10 \mathrm{~W}$ was $\sim 1000^{\circ} \mathrm{C}$; for the niobium alloys, the maximum temperatures were $<400^{\circ} \mathrm{C}$. For the aluminum materials, the maximum exit temperature was constrained to be about $120^{\circ} \mathrm{C}$.

The aluminum materials were originally included in this study specifically because of their low induced activity. However, it appears that the rate of diffusion of the neutron-produced vacancies to the grain boundaries and the subsequent void formation is in inverse proportion to the melting point of the mateerial. ${ }^{\mathrm{J} 3}$ Consiquently, the useful lifetime of structures made of the lower melting point materials may be shorter than for high melting point materials.

\section{EFFECT OF PARAMETER VARIATIONS}

The parameters chosen for the reference design ( $L=1 \mathrm{~m}, \mathrm{D}=10 \mathrm{~mm}, \delta=0.5 \mathrm{~mm}, \mathrm{p}_{\text {in }}=280 \mathrm{psi}$ ) are not optimized; they were simply chosen as reasonable first guesses for first-wall panel dimensions in the highest heat flux areas.

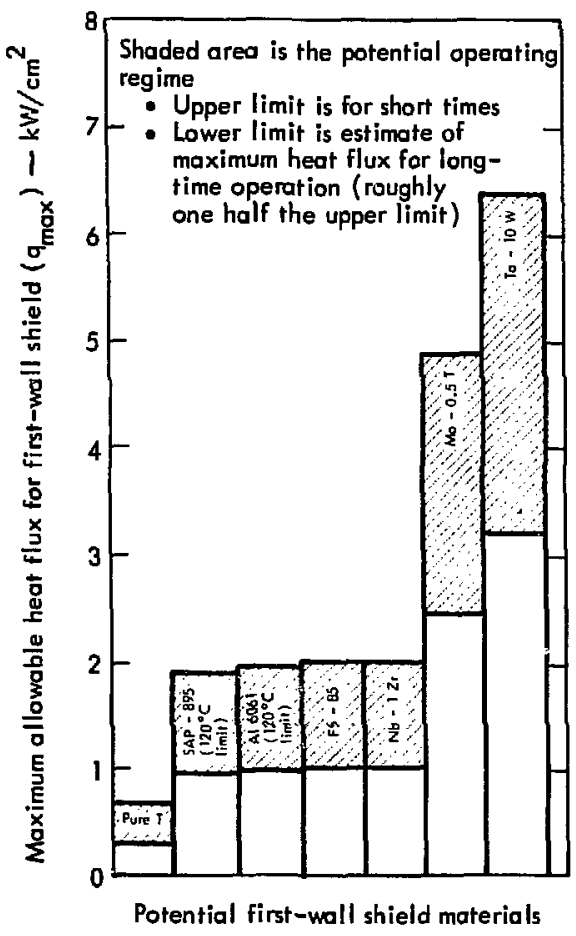

Fig. J-12. Estimates of the short-time and long-time heat-flux capability of various tube-wall materials for the reference case with water coolant $(\Delta \mathrm{p} / \mathrm{p}$ constrained to be 0.5 for all cases).

While no full optimization study has been performed, several calculations were made in which the tube dimensions and the inlet water pressure were varied. For example, thinner tube walls increase the heat flux capability as shown in Fig. J-8 because the thermal stresses always dominate at the high heat fluxes. Longer tube lengths, larger tube diameters, and higher water pressures were found to decrease the maximum heat flux capability somewhat for a fixed fractional pressure drop of 0.5. Fusther studies to find optimized designs for specific applications are certainly warranted.

\section{CONCLUSIONS}

A tubular wall of refractory metal alloys such as Ta-10W can probably handle energy fluxes from the 
plasma as high as $6.4 \mathrm{~kW} / \mathrm{cm}^{2}$ if high-velocity water is used as the coolant. This value is for a specific geometry (L- $1 \mathrm{~m} . \mathrm{D}=10 \mathrm{~mm}, \delta=0.5 \mathrm{~mm}$ ) and inlet water pressure (280 psi). For Mo-0.5Ti under the sane conditions, the maximum value is about $5 \mathrm{~kW} / \mathrm{cm}^{2}$. The niobium alloys and aluminum materials have maximum values of about $2 \mathrm{~kW} / \mathrm{cm}^{2}$. Other materials, vanadium, the titanium alloys, and the stainless steels hid hcat flux capabilities below $1 \mathrm{~kW} / \mathrm{cm}^{2}$ for this application and geometry.

When the fractional pressure drop in the tubes is heid at 0.50 , the pumping power is about $380 \mathrm{~kW} / \mathrm{m}^{2}$ of first-wall area subjected to the high haat fluxes. Substantially lower values of pumping power can be obtained by a relatively small reduction in the maximum allowable heat flux. For example, the pumping power can be reduced by a factor of three with only a 5-10\% reduction in the allowable heat flux.

It should be roted that there exist some important uncertainties in the analysis, e.g., the critical heat flux under these conditions and the neutron damage effects which require experimental studies.

Even though these maximum heat flux results are not optimized values, they are sufficiently high to indicate that water-cooled first walls for FERF and other early experimental fusion machines can probably be designed with some margin of safety for the unexpected.

\section{ANNEX 1 - EQUATIONS USED IN THIS STUDY}

The allowable working stress of the various materials was approximated by

$\sigma_{\text {allow }}=f_{\text {allow }} \sigma_{y}=f_{\text {allow }}\left(A_{1}-A_{2} T_{\text {wo(ex }}\right)$,

where $\sigma_{y}=A_{1}-A_{2} T_{\text {wo(ex) }}$,

and $f_{\text {allow }}$ is the inverse of the safety factor.

The coefficients $A_{1}$ and $A_{2}$ were chosen to give a good fit to the tensile yield stress data near $\boldsymbol{T}_{\text {wo(ex) }}$. the tube-wall temperature at the hottest point of the outer surface at the tube exit. (This is the hottest temperature of the tube material for the simplified case of uniform axial heat flux assumed in this study as shown in Fig. J-5. Work is now in progress on a generalized study which includes axial heat-flux variations.)

The total stress in the tube wall at the exit has been estimated as the sum of the hoop stress and thermal stress; that is,

$$
\sigma_{\text {tot }} \approx \sigma_{\text {hoop }}+\sigma_{\text {th }} \text {, }
$$

where $\sigma_{\text {hoop }} \simeq \frac{P_{\mathrm{ex}}(\mathrm{D})}{2 \delta}$,

and $\sigma_{\text {th }} \simeq \frac{\alpha E}{2(1-v)} \Delta T_{w}$

These relations are valid for thin-walled tubes where $\delta / D<<1$. The material property data required to calculate $\sigma_{\text {th }}$ were evaluated at the estimated value of $T_{\text {wo(ex) }}$ for the maximum allowable heat flux for each material. These property data are listed in

Table J-2.

The temperature drop across the tube wall has been calculated for the peak value of heat flux at the point on the circumference of the tube facing the incoming energy flux, $q_{\text {peak }}=q\left(\theta=0^{\circ}\right)$, as

$$
\Delta T_{w}=T_{w o(e x)}-T_{w i(e x)} \cong \frac{{ }^{q_{\text {peak }}}{ }^{\delta}}{k_{w}} \text {. }
$$

Note: As mentioned previously, the heat flux has been assumed to be uniform along the length of the tube, $L$, in these calculations. As shown in Fig. J-I3, it is assumed to vary only circumferentially. As a result, $\Delta T_{w}$ at a given $\theta$ is constant along the length of the tube.

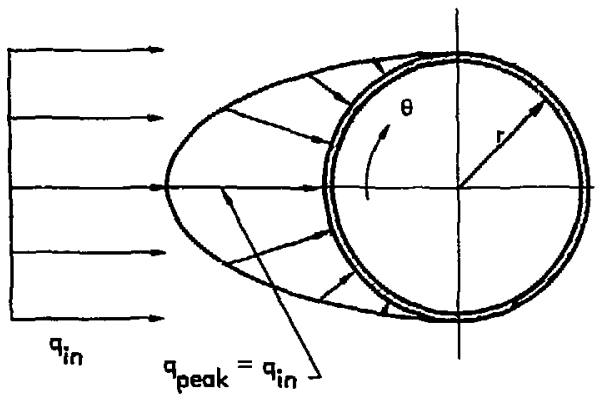

Fig. J-13. Circumferential theat-flux distribution. 


$$
\Delta p=p_{i n}-\bar{z}_{e x}=f \frac{L}{D_{i}} \frac{G^{2}}{2 \rho g_{c}} .
$$

where $f$ is given by Eq. (J17).

\section{ANNEX 2 - METHOD OF SOLUTION FOR THE MAXIMUM ALLOWABLE HEAT FLUX}

This set of equations, Eqs. (J9) through (J18), has been solved for the special case of greatest immediate interest, the maximum allowable peak heat flux, $q_{\max }$, by equating the total stress to the allowable stress; i.e. (see Fig. J-14),

$$
\sigma_{\text {tot }}=o_{\text {allow }}
$$

Using Eqs. (J9) through (J12), we obtain one equation relating $T_{w o(e x)}$ to the maximum allowable peak heat flux, $\mathrm{q}_{\text {peak }}$, so that

$B_{1}+B_{2} q_{\text {peak }}=f_{\text {allow }}\left(A_{1}-A_{2} \times T_{\text {wo(ex })}\right.$.

where the constants are functions of material properties, tube dimensions, and $p_{\mathrm{ex}}$.

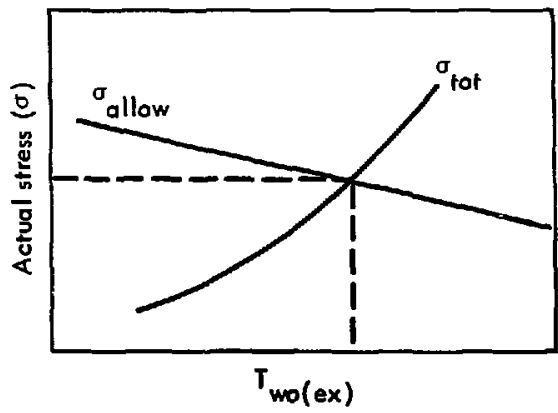

Fig. J-14. Allowable stress variations with peak tube-wall temperature.

The exit pressure, $p_{\text {ex }}$, can be eliminated as an unknown by specifying $\left(\Delta p / p_{\text {in }}\right) \triangleq\left(p_{\text {in }}-p_{\text {ex }}\right) / p_{\text {in }}$. (Typically, a value of 0.5 has been used for the fractional pressure drop.) Therefore,

$$
P_{\text {ex }}=P_{\text {in }}\left(1-\frac{\Delta p}{P_{\text {in }}}\right)
$$

Also, $G$ is evaluated from the specified values of $\Delta p$ and $\mathrm{p}_{\text {in }}$ using Eq. (J18).

A second equation relating $T_{\text {wo(ex) }}$ to the peak heat flux, $\mathrm{q}_{\text {peak }}$, is obtained using Eqs. (J12) to (J17) so that

$$
\begin{aligned}
T_{w o(e x)} & =T_{B(i n)}+\Delta T_{B}+\Delta T_{\text {film }}+\Delta T_{w} \\
& =C_{3}+\left(C_{4}+C_{5}+C_{6}\right) q_{\text {peak }},
\end{aligned}
$$

where the $C^{\prime} s=f\left(L, D_{i}, C_{\text {asym }}, C_{p}, G, P_{r} f, k_{w}\right.$, $\delta$ and $\left.\mathrm{T}_{\mathrm{b}(\mathrm{in})}\right)$

Eqs. (J20) and (J22) are two equations in the two unknowns, $T_{\text {wo(ex) }}$ and $q_{\max }$. Solving first for $\eta_{\max }$ gives

Maximum allowable $\mathrm{q}_{\text {peak }} \triangleq \mathrm{q}_{\max }$

$$
=\frac{C_{1}-C_{2} C_{3}-B_{1}}{B_{2}+C_{2}\left(C_{4}+C_{5}+C_{6}\right)} \text {. }
$$

where $C_{I} \triangleq f_{\text {allow }} A_{I}$,

and $C_{2} \triangleq f_{\text {allow }} A_{2}$

Then the corresponding $T_{\text {wo(ex }}$ can be solved for using either Eq. (J20) or Eq. (J22).

Note: No attempt was made to choose an optimum operating pressure, $p_{i n}$, or tube-wall thickness, $\delta$, in this initial study. Work is now in progress on this refinement.

To assure that $\mathbf{T}_{\mathbf{b}(\mathrm{ex})}$ was below the temperature for the onset of nucleate boiling, $T_{\text {onb }}$, an empirical equation for $\mathrm{T}_{\text {onb }}$ from Tong $\mathrm{JS}$ was used:

$$
\begin{aligned}
& \underset{\text { (ex) }}{T_{\text {onb }}} \approx \underset{\text { ex }}{T_{\text {sat }}}\left(p_{\text {ex }}\right)+\Delta T_{\text {J\&L }}-\Delta T_{\text {film' }}, \\
& \text { where } \\
& \underset{(e x)}{\Delta T_{\text {J\&L }}}=60 \mathrm{e}^{-p_{\text {ex } / 900}}\left(\frac{q_{\text {max }}}{10^{6}}\right)^{1 / 4}
\end{aligned}
$$

and

$$
\begin{aligned}
& {[p]=\text { psia }} \\
& {[q]=\frac{\text { Btu }}{h \cdot \mathrm{ft}^{2}}}
\end{aligned}
$$




$$
\left[\Delta \mathbf{T}_{J \& \mathbf{L}}\right]={ }^{\circ} \mathbf{F}
$$

$T_{b(e x)}$ was then calculated from Eq. (J13) and compared to $T_{\text {onb }}$ to verify that

$$
\mathrm{T}_{\mathrm{b}(\mathrm{ex})} \leqslant \underset{(\mathrm{ex})}{\mathrm{T}_{\text {onb }}}
$$

A second check was then performed to verify that $\mathrm{q}_{\max }$ was less than the critical (bunout) heat flux, $\mathrm{q}_{\text {crit }}$. An empirical correlation for $\mathrm{q}_{\text {crit }}$ developed by Rousal ${ }^{\text {l }}$ was used because his correlation is based on Aerojet data for water at high heat-flux levels and high mass flow rates very near the flow regine in this present study. At the tube exit,

$$
\mathrm{q}_{\text {crit }}=\mathrm{D}_{1}+\mathrm{D}_{2} \mathrm{u} \Delta \mathrm{T}_{\text {sub(ex) }},
$$

which is valid for $u \Delta T_{\text {sub }} \gg 5000 \mathrm{ft} \cdot{ }^{\circ} \mathrm{F} / \mathrm{s}$,

where

$$
\Delta T_{S U B(e x)} \triangleq T_{S A T(e x)}-T_{B(e x)} .
$$

The values of $D_{1}$ and $D_{2}$ in Eq. (J26) for water are then

$$
\begin{aligned}
& D_{1}=5.1 \frac{\text { Btu }}{\text { in. }^{2} \mathrm{~s}} \\
& D_{2}=0.000860 \frac{\left(B t u / i n \cdot{ }^{2} \cdot \mathrm{s}\right)}{\left(\mathrm{ft} \cdot{ }^{\circ} \mathrm{F} / \mathrm{s}\right)} .
\end{aligned}
$$

\section{ANNEX 3 - EXAMPLE CALCULATION}

For an illustrative example, assume that we specify the following input data:

$$
\begin{aligned}
& \mathrm{L}=1 \mathrm{~m}, \mathrm{D}_{\mathrm{i}}=10 \mathrm{~mm}, \delta=0.5 \mathrm{~mm} \\
& \mathrm{P}_{\mathrm{in}}=280 \text { psia. } \Delta \mathrm{p}=140 \mathrm{psia} \\
& T_{b(\mathrm{in})} \approx 0^{\circ} \mathrm{C}
\end{aligned}
$$

Calculations then procede as follows: conservative total operating stress

$$
\left.\sigma_{\text {tot }}=1 / 5 \sigma_{y} \text { (i.e., fallow }=1 / 5\right)
$$

(This choice is also based on the fact that it yields a maximum heat flux of about $3 \mathrm{~kW} / \mathrm{cm}^{2}$.)

Solve $E_{q}$. (J23) for the maximum allowable value of the peak heat flux for this stress limit.

$$
q_{\max }=3.1 \mathrm{~kW} / \mathrm{cm}^{2}
$$

From Eq. (J20), the maximum tube wall temperature at the exit is

$$
T_{\text {wo(ex) }}=467^{\circ} \mathrm{C}\left(872^{\circ} \mathrm{F}\right)
$$

From Eq. (J9) the corresponding stress is

$$
\sigma_{\text {tot }}=\sigma_{\text {allow }} \approx 23,900 \mathrm{psi} \approx 24,000 \mathrm{psi}
$$

We find $\sigma_{\mathrm{y}} \approx 120,000 \mathrm{psi}=S \sigma_{\mathrm{allow}}$ at a temperature of $467^{\circ} \mathrm{C}$.

The stress components given by Eqs. (J10) and (JII) are

$$
\begin{aligned}
& a_{\text {hoop }}=1400 \mathrm{psi} \\
& a_{\text {th }} \approx 22,500 \mathrm{psi}
\end{aligned}
$$

We sce that the thermal stress tends to dominate the hoop stress for this situation where we are trying to maximize the allowable heat hux.

Using the pressure drop Eq. (J18) and eliminating the friction coefficient using Eq. (J17), solve next for the mass flow rate parameter, $G$ :

$$
G=4.31 \therefore 10^{4} \frac{\mathrm{kg}}{\mathrm{m}^{2} \mathrm{~s}}\left(31.7 \times 10^{6} \frac{\mathrm{lbm}}{\mathrm{h} \cdot \mathrm{ft}^{2}}\right)
$$

The conesponding values of the friction and heat transfer cuefficients are

$$
\begin{aligned}
& \mathrm{f}=0.0092 \\
& \mathrm{~h}=2.07 \times 10^{5} \frac{\mathrm{w}}{\mathrm{m}^{2} \therefore \mathrm{c}},
\end{aligned}
$$

Assume that we use Ta-10W alloy and choose a 
where $R_{\mathrm{e}} \approx 3.2 \times 10^{6}$ and the Prandl number $\approx 1.0$ for an estimated mean film temperature at the tube midpoint of about $180^{\circ} \mathrm{C}\left(360^{\circ} \mathrm{F}\right)$.

Next, calculate the corresponding temperature differences from Eqs. (J12), (J13) and (J15):

$$
\begin{aligned}
& \Delta T_{w}=250^{\circ} \mathrm{C} \\
& \Delta T_{B}=22^{\circ} \mathrm{C} \\
& \Delta T_{\text {film }}=195^{\circ} \mathrm{C}\left(\text { for } C_{\text {usym }} \approx 1.3\right)
\end{aligned}
$$

Using these values of the temperature differences, calculate the following temperatures:

$$
\begin{aligned}
& \mathrm{T}_{\mathrm{B}(\mathrm{ex})}=22^{\circ} \mathrm{C}\left(71^{\circ} \mathrm{F}\right) \\
& \mathrm{T}_{\text {wi(ex) }}=217^{\circ} \mathrm{C}\left(422^{\circ} \mathrm{F}\right) \\
& \mathrm{T}_{\text {wo(ex) }}=467^{\circ} \mathrm{C}\left(872^{\circ} \mathrm{F}\right) \text { as given previously. }
\end{aligned}
$$

In addition, calculate the mean film tempersture (at the tube midpoint):

$$
\begin{aligned}
T_{F(\text { mean })} & \triangleq \frac{T_{B(\text { mean })}+T_{\text {wi(mean })}}{2}-\frac{}{109^{\circ} \mathrm{C}\left(228^{\circ} \mathrm{F}\right)}
\end{aligned}
$$

Since this value is lower than the carlier estimated value of $180^{\circ} \mathrm{C}\left(360^{\circ} \mathrm{F}\right)$, we should change the water property data and then iterate until we achieve agree-

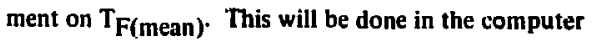
code now being developed, but it was not done in these initial hand calculations.

Next, calculate the temperature for the onset of fully developed nucleate boiling $\left(T_{\text {onb }}\right)$ from Eq. (J24):

$$
\begin{aligned}
T_{\text {(onb)(ex) }} & =T_{\text {sat(ex) }}+\underset{\text { (ex) }}{\Delta T_{\text {J\&L }}-\Delta T_{\text {film }}} \\
& =179^{\circ} \mathrm{C}+50^{\circ} \mathrm{C}-195^{\circ} \mathrm{C} \\
& =34^{\circ} \mathrm{C}\left(93^{\circ} \mathrm{F}\right)
\end{aligned}
$$

Since $T_{b(e x)}=22^{\circ} \mathrm{C}$, this approximatc equation implies that there is not fully developed subcooled nucleate hoiling.

As an alternate method of checking, compare the inner tube-wall temperature to the saturation temperaiure plus $\Delta \mathrm{T}_{\mathrm{J} \& \mathrm{~L}}$ :

$$
T_{\text {wi(ex) }}=217^{\circ} \mathrm{C}<T_{\text {sat(ex) }}+\Delta T_{\text {J\&L(ex) }}=229^{\circ} \mathrm{C}
$$

This implies that we are below the onset of fully. developed subcooled nucleate boiling. However, iute that

$$
T_{\text {wi(cx) }}=217^{\circ} \mathrm{C}>T_{\text {sat(ex })}=179^{\circ} \mathrm{C}
$$

This implics that there will be a small amount of partial nucleate boiling. Figure $\mathrm{J}-15^{\mathrm{J} 6}$ shows the typical qualitative relationship between surface heat flux and surface temperature. Our operating point is in the partial subcooled boiling regime between points $B$ and $E$. This small amount of subcooled nucleate boiling should have little effect on the temperatures and $\Delta \mathbf{p}$.

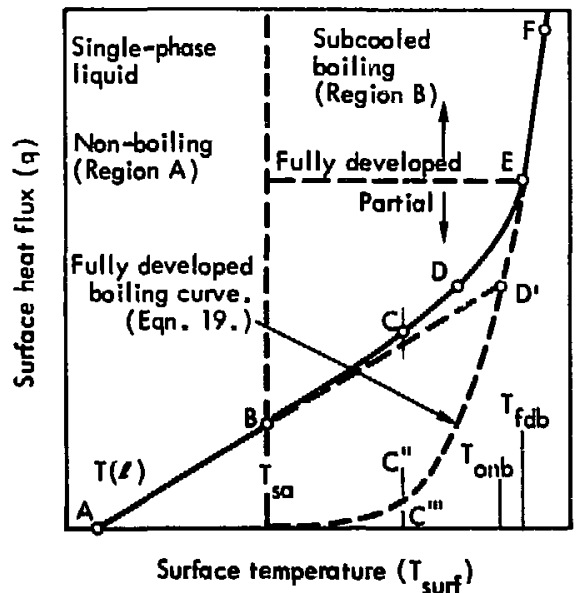

Fig. J.15. Typical quali1 ative curve describing tle variation of surface lieat flux with surface temperalure for subcooled boiling (1aken from Fig. 8, page 976, in Reference J6).

Note that the Jens and Lottes empirical equation may not be accurate for these flow conditions. Consequently, additional experimental work is necessary 
to verify the $\Delta T_{\mathbf{J} \& \mathbf{L}}$ at high heat fluxes and subcooling.

Finally, check the critical (burnout) heat flux using the Rousar correlation (Eq. (J26)) which is valid because

$$
u \Delta T_{\text {sub(ex) }} \approx 39,000 \gg 5000 \frac{\mathrm{ft} \cdot{ }^{\circ} \mathrm{F}}{\mathrm{s}}
$$

Thus,

$$
\mathrm{q}_{\mathrm{crit}} \approx 7.2 \mathrm{~kW} / \mathrm{cm}^{2}
$$

This is much greater than our calculated maximum allowable heat flux of about $3.1 \mathrm{~kW} / \mathrm{cm}^{2}$ for our chosen structural safety factor of 5 .

\section{REFERENCES}

J1. D. C. Rousar, Correlation of Burnout Heat Flux for Fluids at High Velocity and High Subcooling Conditions, M.S. Thesis, Univ, of California, Javis (1966)

J2. W. M. Rohsenow and J. P. Hartnett, Eds. Handbook of Heat Transfer, (McGraw-Hill, 1973), Ch. 13.

J3. A. Mukhergee, Dept. of Mechanical Fngineering, Univ. of California, Davis, private communication.

J4. W. C. Reynolds, Int. J. Heat Mass Transfer 6, 445 (1963).

J5. L. S. Tong, Boiling Heat Transfer and TwoPhase Flow (Wiley, 1965).

J6. J. G. Collier and G. B. Wallis, Two-Phase Flow and Heat Transfer, Vol. III, Summer Cc'rse Notes, Stanford, (1967). 


\section{Appendix K - Building Complex}

The FERF reactor building is a highly specialized concrete structure. Its design is integrated with the design of the reactor systems, the specimen manufacturing facilities, the specimen examination facilities. and the tritium handling systems.

\section{BUILDING FUNCTIONS}

The building will provide the following:

- Shelter space for the reactor and allied equipment (including maintenance and operating equipment).

- Radioactive contamination control and protection of personnel from excessive amounts of radjation.

- A containment vault that will retain released radioactive materials and gases resulting from the reactor or its associated equipment.

- Facilities for the safe collection and disposal of iritium and contaminated wastes so that the hazard to personnel operating the facility will be minimized.

- Work areas for fabrication of specimens, for examination of irradiated samples, for certain machining operations, and for storage.

- Tritium recovery and storage facilities.

- Utilities: of íve space, and shops as required to meet the minimum needs of aperation and maintenance.

\section{DESCRIPTIONS OF PRINCIPLE AREAS}

Building plans, sections, and elevaiions are shown in Figs. $\mathrm{k}-1$ through $\mathrm{K}-5$.

\section{Peactor Containment Vault}

The reactor containment vault provides a housing for the reactor, biolcgical shielding, and a gas-tight vessel for any radioactivity or particles released in the event of an accident. It is constructed of reinforced concrete, with walls and roof approximately 10-feet

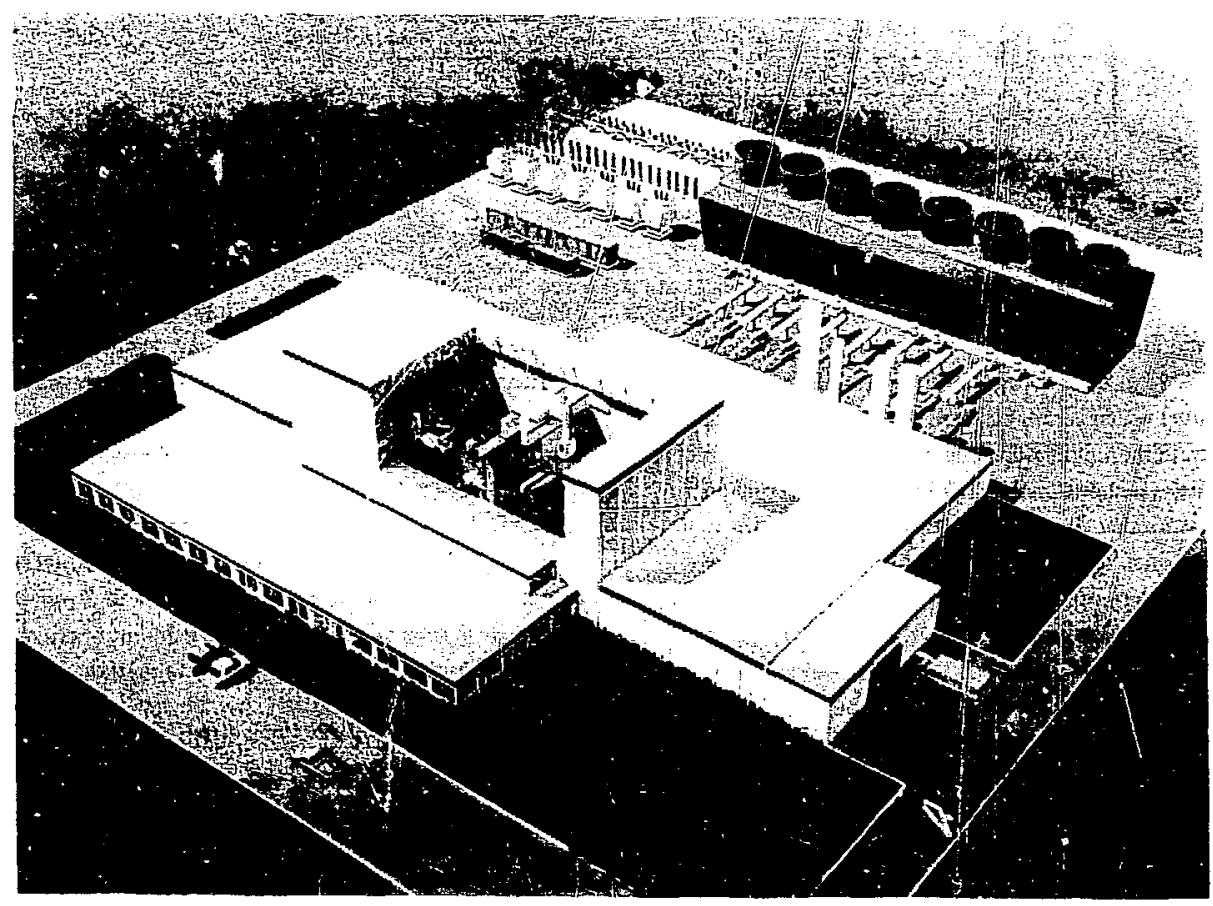

Fig. K-1. Astist's cunception of completed FERF. 


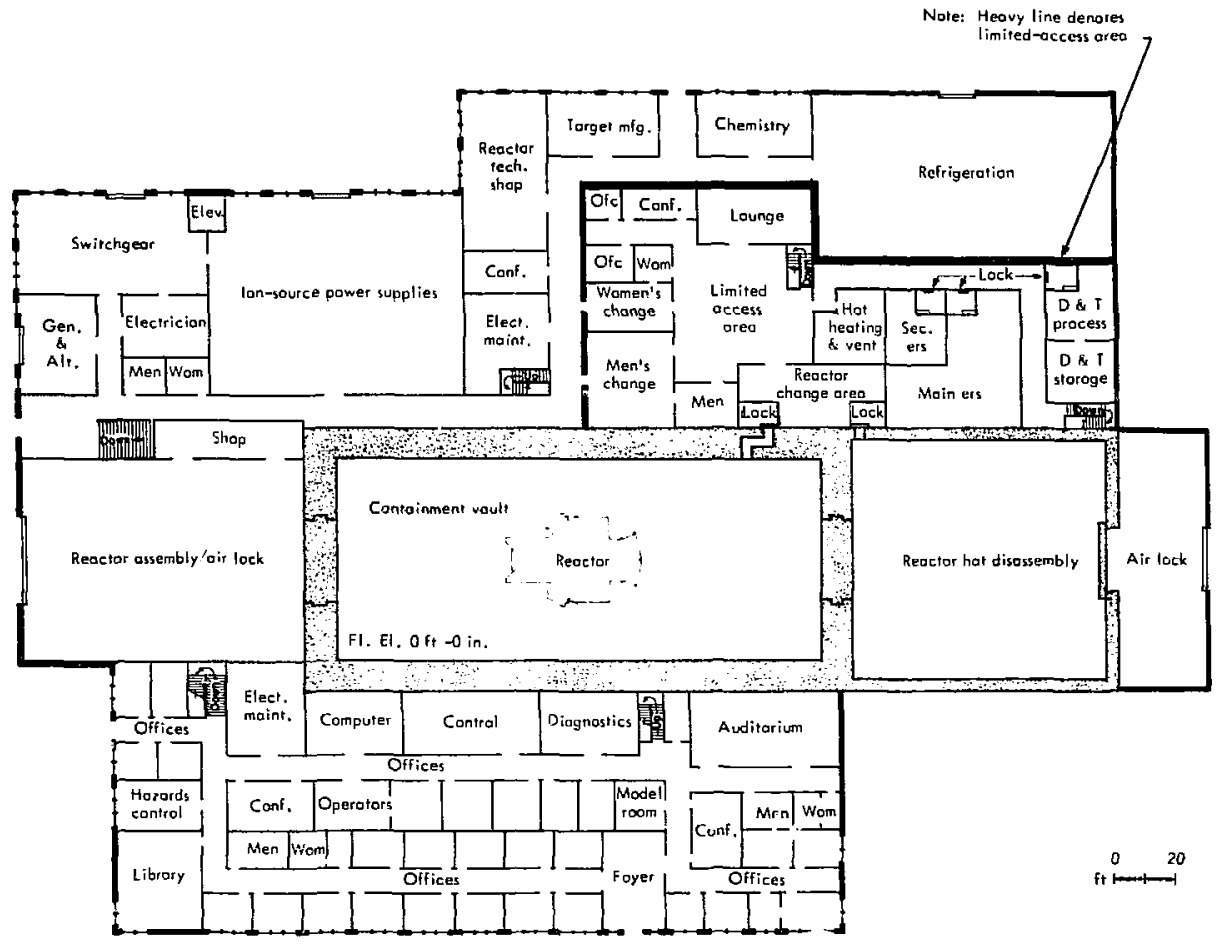

Fig. K-2. Pian view of main tloor.

thick. Inside, the vault is 152 -feet long, 64-feet wide and 60 -feet high. To assure gas-tight integrity, the inside of the vault is sheathed with 18-gauge stainless steel which is suitably sealed to all ducts which penetrate the vault. All cables or pipes within these ducts are sealed or otherwise closed in a positive manner to assure that the vault is leak-tight. The reactor vault is designed to withstand a positive pressure of $2 \mathrm{psi}$ above atmospheric; however, it is normally operated at a negative pressure.

Two vertical, hydraulically operated, gas-tight shieiding doors are provided for equipment access at either end of the vault. Personnel enter and leave the vault via an air lock which leads from a limited-access area.

A 50-ton bridge crane with a shielded control cab traverses the entire vault area and is used to assemble and disassemble the reactor. This crane can also be remotely operated from the manipulator gallery and the operation viewed through shielding windows.

The reactor vault is operated at a negative pressure and contains an inert gas such as nitrogen. This inert atmosphere is continuously circulated through highefficiency filters to remove radioactive contamination.

A leak test is performed periodically to assure that there are neither leaks nor penetrations in the structure. For this test, the vault is pressurized to about $2 \mathrm{psi}$ and the leak rate is determined over a 24-hour period using the absolute pressure-temperature msthod.

\section{Reactor Assembly and Air Lock Area}

A reactor staging and assembly area is located at one end of the reactor vault. Here, parts of the reactor can be preassembled before final assembly in the reactor vault. Preassembled components are transferred to the reactor vault through the shielding 


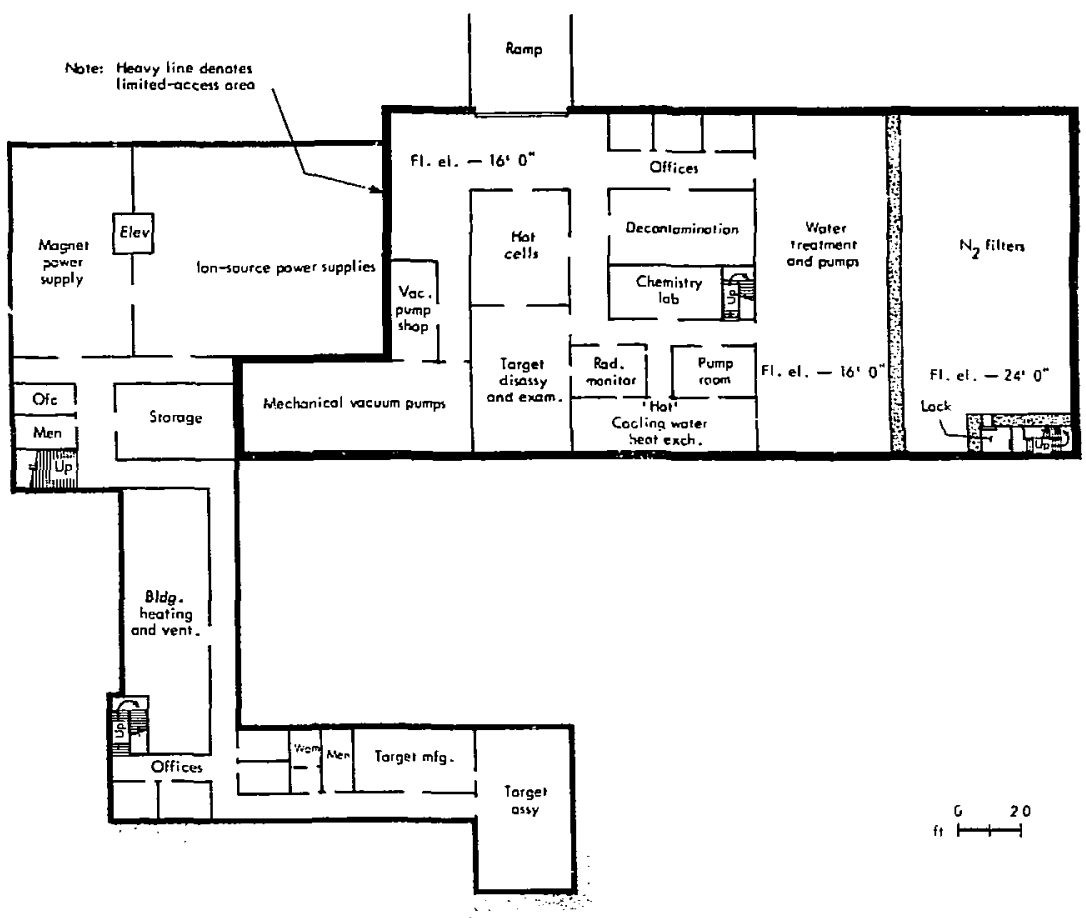

Fig. K-3. Plan view of basement.

door on a transfer dolly or truck. The reactor assembly area is air-tight so that it can serve as an air lock when the shielding door to the reactor vault is open. A technician's shop and tool crib is located adjacent to the assembly area.

\section{Reactor Hot Disassembly Area}

At the opposite end of the reactor vault from the assembly area is a reinforced concrete extension which forms a shielded reactor-disassembly area. Radioactive reactor components are transferred through the shielding door by truck or dolly for disassembly, decontamination, and/or examination. A sliding, shielding door communicates with an air lock which provides the means for removing parts from the area without compromising the atmosphere of the disassembly area. A personnel air lock connects the disassembly area with the limited-access area.

\section{Reactor Viewing Gallery}

Reactor maintenance operations can be observed remotely from two peripheral viewing galleries which are situated on the second floor. Remote manipulators controlled from this area are used to insert specimens in the blind specimen tubes. The wall and window shielding is designed to permit continuous personnel occupancy.

\section{Administration and Control area}

The administration and control wing is a singlestory structure of approximately $18,250 \mathrm{ft}^{2}$ and contains the following:

- Reactor control room: provides space for operating consoles, computer terminals, and closedcircuit television monitors for viewing various parts of the reactor system.

- Computer room: houses computer and associated 


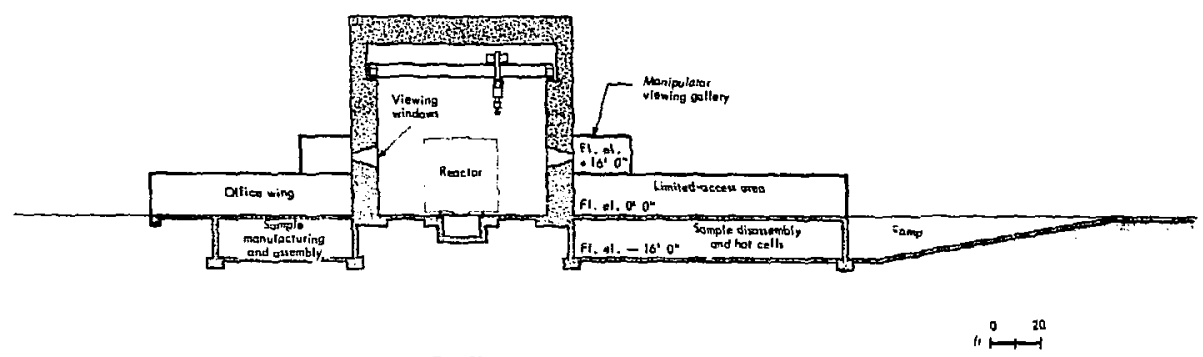

Fig. K-4. Transverse cross-sectional view.

equipment

- Diagnostics room: houses the remote data collecting and counting equipment.

- Offices

- Library

- Auditorium

- Conference rooms

- Electronic maintenance shop: provides facilities for maintenance of the computer, control, and diagnostics equipment

- Toilet facilities

- Lobby

\section{Target Manufacture and Assembly Areas}

The target manufacture and assembly rooms are located in the basement of the administration and control wing. This area is used to prepare and instrument the samples and specimens that enter the through-reactor specimen tubes. These through tubes terminate on the wall of the target assembly room and lead dirertly to the reactor.

\section{Shops}

Small assembly and maintenance shops are located throughout the building. In addition, a second targetmanufacturing shop is located on the first noor adjavent to the entrance to the limited-access area that leads to the reactor vault. Samples for the blind specimen tubes that terminate on the side of the reactor are prepared in this shop.

\section{Power Supply Areas}

Rooms on the first floor and basement level house the following electrical equipment and power supplies:

- Emergency generator.

- 400-Hz alternator for ion source filament.

- Power supplies for ion source.

- Distribution switchgear.

In general, all power supplies will be water-cooled to minimize the ventilation requirements in these areas.

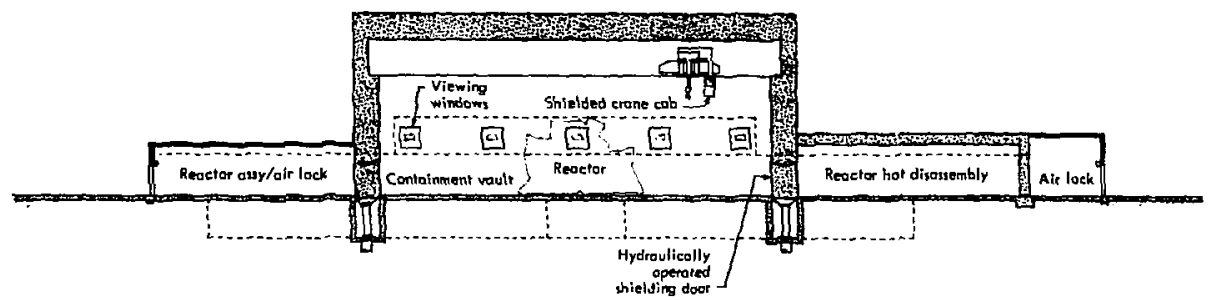

20

Fig. K-S. Longitudinal cross-sectional view. 
Mechanical Equipment Areas

There are two mechanical equipment areas. A conventional one houses eciuipment for building heating. ventilation, and reactor refrigeration. A second, limited-access area houses potentially radioactive equipment which inclutes the following:

- Mechanical vatuum pumps.

- Fluid heat exchangers and pumps for cooling specimens.

- Treatment facilities and pumps for the water coolant.

- Heating and ventilation equipment for the limited-ar,iess area.

Limited-Access Area

A limited-access area on the first floor and basement levels is provided for operations and equipment that are radioactive or potentially radioactive. Two change areas provide entry. One group of change rooms contains lockers and clothing bins, toilet and shower facilities, and personnel decontamination and monitoring facilities. Shielding and life-support suits are located in a second change area that provides entry to the reactor containment vault and the reactor hotassembly area.

Much of the space in the limited-access area is used for the nitrogen filters and the tritium recovery and storage equipment described in Appendix G.

The through specimen tubes terminate in the target disassembly and examination room. An area next to this room contains shielded "hot" cells or compartments which have viewing windows and manipulators. Irradiated specimens are disassembled and examined in these shielded compartments. A monorail system handles casks containing radioactive samples. The irradiated specimens are analyized in an analytical chemistry laboratory located near the target disasembly room.

\section{Cooling Towers}

The reactor power which has been dissipated in the heated, low-conductivity cooling water is transferred to the atmosphere. The water is passed through heat exchangers that transfer the heat to a cooling tower. Each cooling-tower/heat-exchanger combination is rated for $12.5 \mathrm{MW}$. Table K-1 lists the important claracteristics of each cooling tower. The total installed capacity for the FERF facility is in excess of $150 \mathrm{MW}$.

Table K-1. Characteristics of a 12.5-MW cooling gower

Cooling tower cells:

$\begin{array}{ll}\text { Capacity each } & 42.500 \mathrm{Btu} / \mathrm{h} \\ \text { Wet bulk } & 70 \text { degrees } \\ \text { Approach } & 5 \text { degrees } \\ \text { Range } & 15 \text { degrees } \\ \text { Flow rate } & 5700 \mathrm{GPM} \\ \text { Fan motor horsepower } & 150 \mathrm{hp}\end{array}$

Heat exchangers: $\quad 12.5$ WM each

Capacity each $\quad 42,500 \mathrm{Btu} / \mathrm{h}$

TW temperature in/out $\quad 75^{\circ} \mathrm{F} / 90^{\circ} \mathrm{F}$

LCW temperature in/out $\quad 105^{\circ} \mathrm{F} / 85^{\circ} \mathrm{F}$

Mean temperature difference, $\Delta \mathrm{t}_{\mathrm{m}} 12.5^{\circ} \mathrm{F}$

Approximate tube surface $\quad 17,000 \mathrm{ft}^{2}$

Shell diameter 45 in.

Tube length $\quad 35 \mathrm{ft}$

TW on tube side/

LCW on shell side

Tower water pumps:

Flow rate each

$5700 \mathrm{GPM}$

Head

$100 \mathrm{ft}$

Motor horsepower

$200 \mathrm{hp}$

LCW pumps:

Flow rate each $\quad 4250$ GPM

Head $275 \mathrm{ft}$

Motor horsepower $\quad 400 \mathrm{hp}$

Deionized water manufacturing:

Capacity

15 GPM

Equipment: filters, ion exchangers, deionized water storage tanks, etc.

LCW polishing and deaeration:

Capacity

$50 \mathrm{GPM}$

Equipment: filters, ion exchangers, vacuum deaerator, pumps, etc.

\section{Electrical Substation}

The installed power capacity of the FERF facility 
substation is 180 MVA. Table $\mathrm{K}-2$ lists the detailed power requirements for the complete installation.

Two 230-kV lines come to the site on a single row of towers. These lines terminate on disconnect switches for isolation and switching. Two separate, three-phase transformer banks are provided, each having three 30-MVA single-phase transformers. One spare, standby transformer can be switched into service to replace any defective transformer. The voltage ratings of the transformers are $230 \mathrm{kV}$ to $13.8 \mathrm{kV}$. Circuit breakers connect each set of transformers to a 13.8-kV bus. A normally open bus tie breaker connects the two $13.8-\mathrm{kV}$ busses.

Outdoor distribution transformers rated $13.8 \mathrm{kV}$ to $2.4 \mathrm{kV}$ provide power to the large loads such as the refrigerators and water pumps. Other outdoor transformers rated $13.8 \mathrm{kV}$ to $480 \mathrm{~V}$ supply power to $480-\mathrm{V}$ switchgear which is housed within the building. The 480-V switchgear is located at approximate centers of load and serve major loads. Local, dry-type, indoor transformers feed panels to supply lighting, communications, alarm equipment, and control.

Two gas-turbine-driven generators provide emergency power. Batteries are provided for switchgear operation, $d$ c. lighting, d.c. instruments, d.c. safety circuits, as. d.c.-to-a.c. inverters.

Table K-2. Facility installed-power requirements.

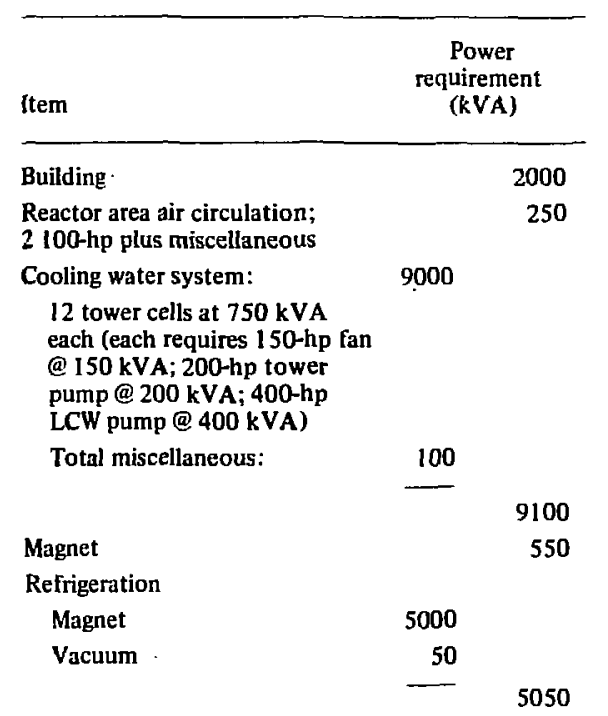

Mechanical vacuum pumps

$$
2-60 \text { hp }
$$

Miscellảneous

\section{$D$ and $T$ recovery}

2 refizgerators; 500 W @

$25 \mathrm{~K}$ each

2 stack blowers; 20 hp each

200

Furnaces

40

Nitrogen gas preheater

Process pumps

Ion source power supplies

Each source (deuterium)

Filament

150

Arc

136

Acceleration

1056

Deceleration

72 deuterium sources @ $1368 \mathrm{kVA}$ each

Each source (tritium)

Filament

Arc

Acceleration

1092

Deceleration

49

40 tritium sources @ 1427

kVA each

Total: 177,976

[ustall: 180,000

(180 MVA)

\section{SITE REQUIREMENTS}

No specific site has been chosen for the FERF facility; however, the cost estimate (Appendix $L$ ) is based on a level site having the following requirements:

- Suitable substrata for massive structures.

- Large amount of available power.

- Adequate pure water supply.

- Easily accessible to supporting industry and commerce and transportation networks.

- Ideal weathe conditions for dispersion of released low-levc. sddioactive gas.

- Access roads, sidewalks, fences, and landscaping. 


\section{Appendix L - Details of Reactor Facilities}

Details of the cost estimate and a discussion of the cost-estimating method are given in Tables $L-1$ through $L-4$. A summary is given in the main body of this report.

Table L-1. Reactor costs.

(Two superconducting coils, including structure, shielding, refrigeration, and power supplies.)

Coils

Superconductor, NiSn, $7.5 \times 10^{8}$ Am @ \$0.015/Am

$\$ 11,250$

Insulation

300

Winding fixtures

100

Winding

2,000

Assembly and installation

1,000

Total coils:

$\$ 14,650$

\section{Coil structure}

Coil tanks, $3.1 \times 10^{5}$ lbs @ \$5.20/lb (including fabrication)

C-clamps, $1.4 \times 10^{6} \mathrm{lbs} @ \$ 3.70 / \mathrm{lb}$ (including fabrication)

5,180

Intercoil structure, $3.4 \times 10^{4} \mathrm{lbs} @ \$ 5.20 / \mathrm{lb}$ (including fabrication)

180

Bolts, fittings, small parts

700

Assembly and installation

Total coil structure:

Coil radiation slielding

Boral, 1/4 inch, $2000 \mathrm{ft}^{2} @ \$ 30 / \mathrm{ft}$ (including fabrication)

60

190

3,120

Stainless steel, $6 \times 10^{5}$ lbs @ \$5.20/lb (including fabrication)

300

Bolts, fittings

50

500

Assembly and installation

Total coil shielding:

Coil thermal shield

Nitrogen-cooled panels, $3.2 \times 10^{3} \mathrm{ft}^{2} @ \$ 20 / \mathrm{ft}^{2}$

Reflective heat shields, $9.6 \times 10^{3} \mathrm{ft}^{2} @ \$ 4 / \mathrm{ft}^{2}$

Fittings and small parts

Installation

Total coil thermal shield:

Coil refrigeration system (including cryopumping) $3200 \mathrm{l} / \mathrm{h}$ helium reirigerator/liquifier $(27,000 \mathrm{CFM}-8000 \mathrm{hp})$

Helium recovery system Includes: 1500-hp, 1800-psi recovery compressors; $2 \times 10^{6} \mathrm{ft}^{3}, 1800$-psi storage tanks; 3500-CFM @ 1800-psi helium purifier; $10^{5} \mathrm{ft}^{3}$ gas bags, atmospheric; 3500-CMF and 1800-psi gas drier. 
Table L-1. (Continued).

\begin{tabular}{|c|c|c|c|}
\hline \multicolumn{2}{|l|}{ Item } & \multicolumn{2}{|c|}{ Thousands of dollars } \\
\hline \multicolumn{4}{|l|}{ Coil refrigeration system (including cryopumping) (Continued) } \\
\hline Liquid nitrogen storage Dewars $\left(5 \times 10^{5} 1\right)$ & & $\$ 500$ & \\
\hline $\begin{array}{l}\text { Installation costs } \\
\text { Includes: transfer lines, recovery lines, liquid nitrogen lines, etc. }\end{array}$ & & 1,000 & \\
\hline Total coil refrigeration system: & & & $\$ 12,300$ \\
\hline \multicolumn{4}{|l|}{ Coil power supplies } \\
\hline \multicolumn{4}{|l|}{ 90-KW power-supply module ( 8 required) } \\
\hline Power supply (installed) & $\$ 22,000$ & & \\
\hline SCR switching and regisistor & 5,000 & & \\
\hline Firing circuits & 3,000 & & \\
\hline Total & 30,000 & & \\
\hline Modules, $8 @ \$ 30,000$ each & 240 & & \\
\hline SCR contactor & 10 & & \\
\hline Total coil power supplies: & & & 250 \\
\hline Total superconducting coils: & & & 40,290 \\
\hline \multicolumn{4}{|l|}{ Injectors (4) } \\
\hline Ion sources $(4 \times 28=112), 112 @ 550,000$ each & & & 5,600 \\
\hline \multicolumn{4}{|l|}{ Ion source power supplies } \\
\hline Filament $60-\mathrm{Hz}$ to $400-\mathrm{Hz}$ converters, $17 \mathrm{MW} @ \$ 100 / \mathrm{kW}$ & 1,700 & & \\
\hline Filament transformers, $112 @ \$ 1500$ each (installed) & 170 & & \\
\hline Arc power supplies, $136 \mathrm{~kW}, 112 @ \$ 27,000$ each & 3,020 & & \\
\hline $\begin{array}{l}\text { Deuterium source, acceleration power supply, } 1056 \mathrm{~kW} \text {, } \\
72 @ \$ 95,000 \text { each }\end{array}$ & 6,840 & & \\
\hline $\begin{array}{l}\text { Deuterium source, deceleration power supply, } 26 \mathrm{~kW} \text {, } \\
72 @ \$ 15,000 \text { each }\end{array}$ & 1,080 & & \\
\hline $\begin{array}{l}\text { Tritium source, acceleration power supply, } 1092 \mathrm{~kW} \text {, } \\
40 @ \$ 98,000 \text { each }\end{array}$ & 3,920 & & \\
\hline Tritium source deceleration power supply & 720 & & \\
\hline \multicolumn{2}{|l|}{ Total ion source power supplies: } & & 17,450 \\
\hline \multicolumn{2}{|l|}{ Base plate and connector assemblies, $4 @ \$ 50,000$} & 200 & \\
\hline \multicolumn{2}{|l|}{ Flapper valves and drives, $4 @ \$ 25,000$} & 100 & \\
\hline \multicolumn{2}{|l|}{ Transition sections, $4 @ \$ 40,000$} & 160 & \\
\hline \multicolumn{2}{|l|}{ Cooling water piping } & 20 & \\
\hline \multicolumn{2}{|l|}{ Assembly and installation } & 100 & \\
\hline \multicolumn{2}{|l|}{ Total injectors } & & 23,630 \\
\hline \multicolumn{4}{|l|}{ First wall } \\
\hline \multicolumn{2}{|l|}{ Inner layer, material and fabrication, $500 \mathrm{lb} @ \$ 100 / \mathrm{lb}$} & 50 & \\
\hline \multicolumn{2}{|l|}{ Outer layer, material and fabrication, $800 \mathrm{lb} @ \$ 100 / \mathrm{lb}$} & 80 & \\
\hline \multicolumn{2}{|l|}{ Vacuum joints } & \multicolumn{2}{|l|}{50} \\
\hline \multicolumn{2}{|l|}{ Diaphragms } & \multicolumn{2}{|l|}{20} \\
\hline \multicolumn{2}{|l|}{ Manifolds } & \multicolumn{2}{|l|}{20} \\
\hline
\end{tabular}


Table L-1. (Continued).

Item

Thousands of dollars

First wall (Continued)

Fittings

$\$ 20$

Assembly and instaliation

Total lïrst wall:

Irradiation facilitıes

Probes, $4 @ \$ 50,000$ each

Blind tubes,80@\$5,000 each

400

Through tubes, $40 @ \$ 20,000$ each

800

Sample carrier,800@\$50 each

40

Cables, fittings

Manifolds and piping

50

Installation

Total irradiation facilities:

Plasma collector plates

Panels, $64 @ \$ 3,000$ each

Supports

Plates, $4 @$ @3,000 each

10

Piping

10

Assembly and installation

50

Total plasma collector plates:

280

Outer tanks

Main tanks, 2, 150,000 lb @ \$5.00/lb

750

Intermediate tanks, 2, 9,000 lb @ $\$ 5.00 / \mathrm{lb}$

50

Expander tanks, 2, 42,000 lb @ $\$ 5.00 / \mathrm{lb}$

210

Flange clamps

200

Miscellaneous parìs

100

Installation

200

Total outer tanks:

1,510

Support structure

Structure

Running gear

300

Drives

400

Installation

200

Total support structure and drives:

1,100

Diagnostics

Probes, $4 @ \$ 50,000$ each

Laser and gear, 2 assemblies@ \$125,000 each

Leakage-flux sensors, $12 @ \$ 10,000$ each

120

Miscellaneous instrumentation

Installation 
Table L-1. (Continued).

Diagnostics (Continued)

Total diagnostics:

Controls and instrumentation

Controls

$\$ 1,000$

Computer

Total controls and instrumentation:

Vacuum system (refrigeration included in coil refrigeration)

Mechanical pumps

Valves

Piping

Cryopumping panels

120

Nitrogen-cooled panels

60

Reflective heat shields

Miscellaneous parts

Installation

Total vacuum system:

Cooling-water distribution system

Models and tests

Facility models

First-wall tests

Model magnet

Sample tube mockup

Remote handling mocrups

Total models and tests:

Spares

First wall (1) (100\%)

lon sources (28) (25\%;

Ion source power supplies (10\%)

Base plate and connecio: assembly (1) (25\%)

Manipulators (25\%)

Total spares: 
Table L-2. Costs of reactor facilities.

Tritium system

Tritium purification system

S 1,670

Tritium-associated shielding costs

100

Containment

Pre-outgassing heriters

$\$ 330$

Main TRS

18,700

Secondary TRS

70

Total containment: $\quad 19,100$

Monitoring and personnel protection

630

Miscellaneous

Total tritium system:

$\$ 21,780$

Ancillary equipment

50-ton crane with shielded cab

100

Shielded vehicles with manipulators, $2 @ \$ 500,000$

1,000

Reactor remote manipulators, $4 @ \$ 200,000$

800

Hot cells, $4 @ \$ 150,000$

600

Hot-cell manipulators, $8 @ \$ 15,000$

120

Sample instrumentation

150

Radiation monitoring and control

140

Shop equipment

200

Viewing windows, $10 @ \$ 85,000$

850

TV equipment

Total ancillary equipment:

1,000

Total reactor facilities construction:

Table L-3. Costs of building and utilities.

Item

Thousands of dollars

Building

Excavation

$\$ 50$

Piling

290

Containment vault and hot disassembly

3,180

Stainless steel wall lining

310

Penetrations (200)

80

Low-bay areas $99602 \mathrm{ft}^{2}$

5,980

Hydraulic shielding doors, 2

$10,390 \quad \$ 10,390$

Electrical substation

230-kV switchgear

650 
Table L-3. (Continued).

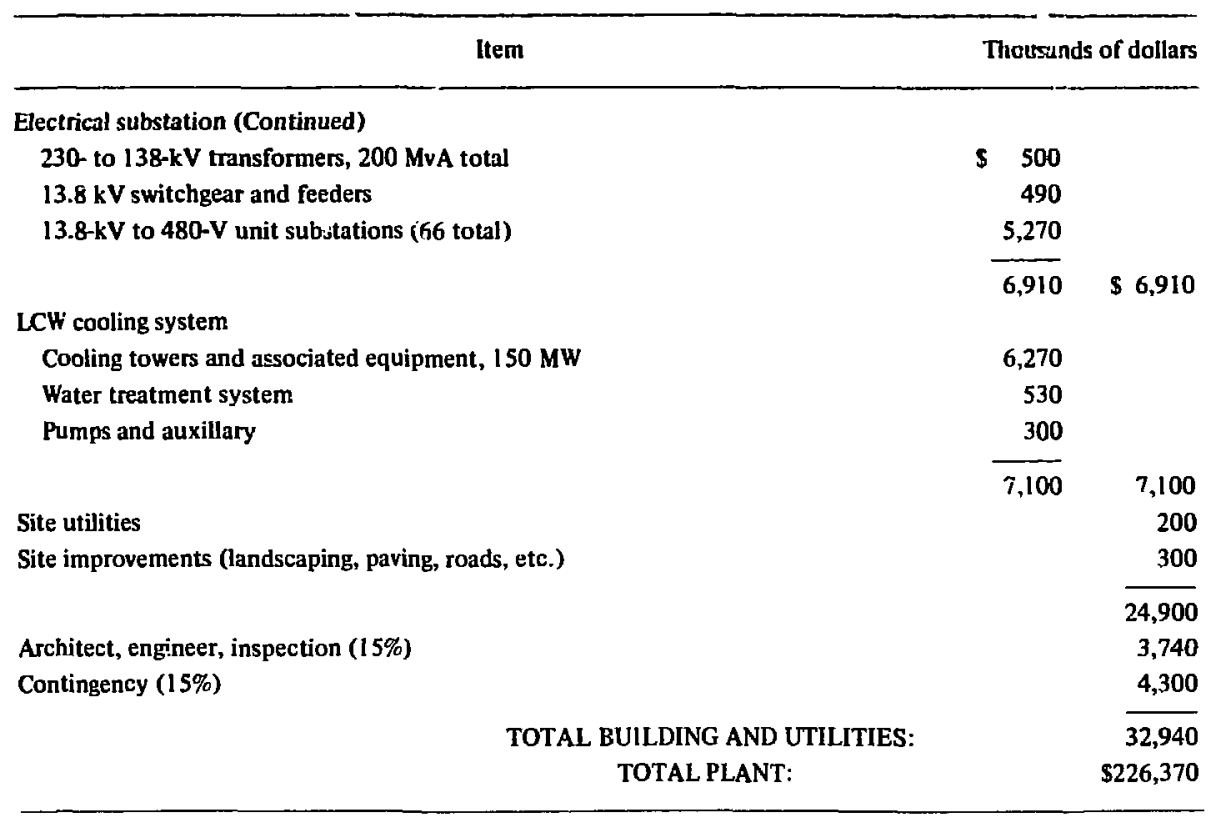

Table L-4. Total plant cost

\begin{tabular}{|c|c|c|}
\hline Item & \multicolumn{2}{|c|}{ Thousands of dollars ${ }^{\mathrm{a}}$} \\
\hline Total reactor construction (from Table L-1) & $\$ 76,420$ & \\
\hline Total reactor facilities construction (from Table L-2) & 26,740 & \\
\hline Total reactor and reactor facilities construction & 103,160 & \\
\hline Engineering, design, inspection, and administration ( $25 \%$ of $\$ 103,160$ ) & 25,790 & \\
\hline Total construction and design & $\overline{128,950}$ & \\
\hline Contingency $(50 \%$ of $\$ 128,950)$ & 64,480 & \\
\hline Total reactor costs & 193,430 & $\$ 193,430$ \\
\hline Total building and utilities (from Table L-3) & 32,940 & 32,940 \\
\hline TOTAL PLANT: & & $\$ 226,370$ \\
\hline
\end{tabular}

a In 1974 dollars 


\section{COST ESTIMATING METHODS}

In making the cost estimate, we have used techniques cicveloped at LLL and LBL during the past decade for estimating costs of large devices such as particle accelerators. Experience gained on these projects has enabled us to refine our procedures white establishing reliable planning factors and high quality standards. The actual construction costs for several accelerators have been compared with the respective estimates, and we now have confidence in the procedures.

The three major methods used in the FERF costs analysis were time-and-material, formula, and projection. A combination of all three was used to derive the cost estimates included here.

\section{Time-and-Material Method}

The time-and-material method for estimating costs is the most conventional and is used on items which are standard and for which a detailed design can be outlined. On items which are used in large quantities, a very accurate unit cost is required so that small errors will not be magnified. For FERF, fixed-price survey quotstions were solicited from a nuniber of commercial firms and the average was used as the estimated cost.

\section{Formula Method}

In the formula method, the cost of a new device is derived by applying a suitable multiplying factor for extrapolation to the known unit cost of a previous, similar device. Adjustments are made for size, quantity, fabrication differences, and esculation. This method is very successful on items for which a conceptual design exists, e.g., vacuum systems, controls powcr supplies, etc.

\section{Projection Method}

In the projection method, an overall cost is obtained by estimating the time and size of the tean required to complete the task. This technique lends itself to development projects and is accurate where the competency of the development projects and is accurate when the competeince of the development group is known from p: evious assignments. 